\title{
Experimental Studies on Bargaining and Competitive Behavior
}

Citation for published version (APA):

Lozano Montana, L. M. (2021). Experimental Studies on Bargaining and Competitive Behavior. [Doctoral Thesis, Maastricht University]. Maastricht University. https://doi.org/10.26481/dis.20210909II

Document status and date:

Published: 01/01/2021

DOI:

10.26481/dis.20210909II

Document Version:

Publisher's PDF, also known as Version of record

\section{Please check the document version of this publication:}

- A submitted manuscript is the version of the article upon submission and before peer-review. There can be important differences between the submitted version and the official published version of record.

People interested in the research are advised to contact the author for the final version of the publication, or visit the DOI to the publisher's website.

- The final author version and the galley proof are versions of the publication after peer review.

- The final published version features the final layout of the paper including the volume, issue and page numbers.

Link to publication

\footnotetext{
General rights rights.

- You may freely distribute the URL identifying the publication in the public portal. please follow below link for the End User Agreement:

www.umlib.nl/taverne-license

Take down policy

If you believe that this document breaches copyright please contact us at:

repository@maastrichtuniversity.nl

providing details and we will investigate your claim.
}

Copyright and moral rights for the publications made accessible in the public portal are retained by the authors and/or other copyright owners and it is a condition of accessing publications that users recognise and abide by the legal requirements associated with these

- Users may download and print one copy of any publication from the public portal for the purpose of private study or research.

- You may not further distribute the material or use it for any profit-making activity or commercial gain

If the publication is distributed under the terms of Article $25 \mathrm{fa}$ of the Dutch Copyright Act, indicated by the "Taverne" license above, 
Experimental Studies on Bargaining and Competitive Behavior 
(C) Lina María Lozano Montaña, Maastricht 2021

All rights reserved. No part of this publication may be reproduced, stored in an automated data system, or transmitted in any form or by any means, electronic, mechanical, photocopying, recording or otherwise, without prior permission of the author.

The author acknowledges financial support from Maastricht University, the Graduate School of Business and Economics at Maastricht University and from the Maastricht University Center for Neuroeconomics (MU-CEN). Funding was also received from New York University Abu Dhabi.

This book was typeset by the author using $\mathrm{BT}_{\mathrm{E}} \mathrm{X}$. The cover art is the work of Jenifer Goméz. 


\title{
Experimental Studies on Bargaining and Competitive Behavior.
}

\author{
Dissertation \\ to obtain the degree of Doctor at Maastricht University \\ on the authority of the Rector Magnificus Prof.dr. Rianne M. Letschert, \\ in accordance with the decision of the Board of Deans, \\ to be defended in public on
}

Thursday, 9th September 2021 at 12:00 hours.

by

Lina María Lozano Montaña 
Supervisor: Prof. Dr. Arno Riedl

Co-Supervisor: Dr. Christina Rott (Vrije University)

Co-Supervisor: Prof. Dr. Ernesto Reuben (NYU Abu Dhabi)

\section{Assessment Committee:}

Prof. Dr. Bart Golsteyn (Chair)

Prof. Dr. Jordi Brandts, Institute for Economic Analysis (CSIC) \& Barcelona GSE Dr. Maria Recalde, University of Melbourne

Dr. Alexander Vostroknutov, Maastricht University 




\section{Acknowledgments}

This dissertation is the outcome of a long journey that started years ago with the decision of jumping into a completely new world. It meant to me being very far from my loved ones for the first time in my life. Also, it challenged me constantly in every possible way. None of this process could have been possible without the support and guidance of many people. I want to thank all of those who walked with me on this road and made this journey bearable for me. My apologies for the long text, but I cannot keep it short when it comes to thanks!

I would like to start by expressing my most sincere gratitude to my supervisors: Professor Arno Riedl, Professor Ernesto Reuben, and Dr. Christina Rott. They did not only mentor and guide me along the way of my PhD, but they also helped me to become confident of my skills and to discover my passion for doing research. I am also deeply thankful to the members of the committee for reviewing my dissertation despite their busy agendas.

Dear Arno, thank you for always believing in me. Since my arrival to Maastricht for the Neuroeconomics Master, you have provided me constant guidance and support to develop all my research ideas. I admire your disposition to discuss research and your sharpness as a researcher. I look back at my time with you as extremely valuable for my professional development. I am grateful you showed me how to enjoy the whole process of creating knowledge. Dear Ernesto, you have been an exceptional mentor to me. I am extremely thankful for having the opportunity to work with you and learn so much from you. Thank you for opening the doors of a new academic world in NYU Abu Dhabi and making me feel like at home there. I cannot wait to continue developing more research projects together and having a lovely time in the desert! Dear Christina, I feel so lucky to have you as my supervisor, mentor, and friend. Thank you for the countless hours of dedication, of discussing research ideas, developing projects together, and for being always present and caring. You make the process of doing research very enjoyable ( $d o$ you remember the time that we almost forgot a subject inside the lab?!). You are such a female role model for me and others. I am sure I will keep learning from you, sharing more projects together and having more boat trips in the Amsterdam canals!

I am very thankful to Elke Lucas for her constant dedication and willingness to help everyone in the department. Her positiveness and smiley face is something I will miss during my breaks. To all the members of our department and the MU-CEN, thank you for your engagement in every 
seminar and for being always willing to give me feedback on my projects, even when they were on a very initial and messy stage. Your feedback was very meaningful to me and I learned a lot from your expertise in behavioral and experimental economics. Among others, I am thankful to Elias Tsakas, Thomas Meissner, Jona Linde, Peter Werner, Sasha Vostroknutov and Matthias Wibral. Thanks to Sylvia Beenen for her efficiency and patience with all my teaching requests; and to Clemens for hiring me as a teacher during the last stage of my dissertation. I am very thankful to the members of the Social Science Division of NYU Abu Dhabi for hosting me several times during my PhD. Especially I want to thank Manu Muñoz, Georgia Michailidou, and all the other postdocs that welcomed me warmly in their department and homes. Many thanks as well to the Department of Economics at Pittsburgh University and especially to my wonderful host Professor Lise Vesterlund for providing very meaningful feedback on my work and for her career advice. I would also like to thank the Social Decision Science Division at Carnegie Mellon University for the inspiring interactions with their faculty members.

I am grateful to all my $\mathrm{PhD}$ colleagues, who did not only shared with me the tough $\mathrm{PhD}$ journey but also made my staying in Maastricht a very pleasant one. Eveline, thanks for being always willing to discuss research ideas, experimental designs, and more importantly, to gossip during our coffee breaks! Hyemi, thanks for bringing always a smile to the office, for the amazing Korean bites, and for providing a lovely home to kitty Luna. Toygarito, thanks for the chilly evenings and for letting me use your cats as a stress release tool - that sounds a bit bad but I promise I never hurt them. Thanks to Nick for all the cozy dinners and for being an amazing host in Arizona. Thanks to Leticia for her kindness and for being a great travel buddy in Shanghai. Henrik, thank you for organizing beer tasting events and for eating cold potatoes at my parties; btw you still owe me a tacos night!. Max, thanks for bringing always good vibes and willingness to party. Thanks to Paul for being a great office mate (even though it was only for a day!) and for his Italian cooking skills. Among others, I also want to thank Giannis, Giang, Anastas, Frauke, Lars, David, Caterina, Riccardo, Evi, Diogo, and Fortuna for being great colleagues and make of our department a very enjoyable one. I also want to thank the talented architect Jenifer Gómez for sharing her artistic skills in the cover art of this book.

My $\mathrm{PhD}$ journey and time in Maastricht wouldn't have been the same without amazing friends I met there. Marcia, I am so lucky that our ways crossed each other. Thank you for your unconditional friendship, our reggaeton nights, and for teaching me so much Puerto Rican slang. I will miss you tremendously my love. Aline, thank you for being such an example of a strong woman and for providing me the best Latino hugs in Maastricht! Thanks to my dearest Dima and Jeanette for our countless nights of laugh, love, and Rock 'n Roll. I will miss you both a lot. Thanks to Fabiolla, Stephan, and Emilio for all the great moments together. I hope we continue the tradition of meeting at least once every summer! Thanks to Anita for being so reliable and my Colombian sister abroad. Thanks to Esztercita, Isa, and Onsem 
for their friendship and amazing moments together. Thanks to Bruna, Carey, and Mirella as well for being so kind to me during our last period in Maastricht.

My PhD journey also meant leaving my beloved home country, Colombia, and being miles away from my dearest friends and family. I want to thank them for being always present during this period and for providing me unconditional support. Thanks to my childhood friends Juani, Pau, and Ale for always being there and for walking with me down this road. Pili, you are my feminist soulmate; I save our memories like treasures and I feel constantly inspired by you. My belleza tropical Diani, thank you for being such a loving friend and always recharging me with positive vibes. Thanks to Ricardo for being always available for my existential crisis and for picking up the phone no matter where he was. Many thanks to David, his support and love were necessary for me to be where I am. Thanks to my family, for always receiving me with tons of love and open arms in Colombia. I am especially grateful to my grandpa Angel R. and grandma Sofía who recently passed away; I wish I could share with both of you this achievement.

Although being far from my loved ones and going through a pandemic was not easy at all, someone very special helped me to cope with all of it. Marcello, thank you for coming to my life and filling it with unconditional love, tons of laughs, and for always encouraging me to do my best. You welcomed me to your world and your family in Caspoggio and made me always feel at home. We make an extraordinary team. I want to continue this road by your hand and develop more personal and professional projects together. I also want to express my gratitude to Marcello's family in Caspoggio. They provided me the coziest environment to finish my dissertation in their lovely homes in the Italian Alps, and made me feel extremely loved and welcomed.

To my mom, Libia, there are not enough words to express how grateful I am for all the sacrifices she has made for me to be here. Thanks for being the rock where my sister and I could always stand firmly on. Without your strength and dedication, none of this would have been possible. Paula, you are the greatest gift my dad could have left me. Thanks for making me a proud sister every single day and for being so damn strong. I love you both more than anything.

To finish, I want to thank someone who I wish with all my heart could read these words, my dad José. You are the most exceptional person I have ever met, thanks for being such an example to me. You taught me to never stop learning, to be a strong and independent woman in a male-dominated world, and to always believe in myself. Thanks daddy for providing me so much love when you were by my side. You gave me so much of it, that I will always feel extremely loved and supported by you. I love you infinitely, and all my achievements will be eternally dedicated to you. Tú eres mi mariposa amarilla. 



\section{Contents}

1 Introduction 1

2 Is there a Preference for Competition? 9

1. Introduction . . . . . . . . . . . . . . . . . 10

2. Literature review . . . . . . . . . . . . . . . . . 12

3. Theoretical framework . . . . . . . . . . . . . . . . . . 14

4. Experimental Design and procedures . . . . . . . . . . . 17

$4.1 \quad$ Experimental Design . . . . . . . . . . . . . . . 17

4.2 Experimental procedures $\ldots \ldots \ldots \ldots . \ldots 22$

5. Results ....................... 22

5.1 Descriptive statistics . . . . . . . . . . . . 23

5.2 Switching behavior . . . . . . . . . . . . . 23

5.3 Measurement of Preferences for Competition . . . . . . 26

5.4 Gender differences . . . . . . . . . . . . . . 30

6. Discussion and conclusions . . . . . . . . . . . 31

A1 Theoretical framework - Additional details . . . . . . 36

A2 Experimental design - Additional details . . . . . . . 36

A3 Analysis separate by treatment . . . . . . . . . . . 39

A4 Analysis whole sample . . . . . . . . . . . . . . 43

A5 Analysis by gender . . . . . . . . . . . . . 44

A6 Instructions . . . . . . . . . . . . . . . 48

3 Estimating Preferences for Competition from Convex Budget Sets 69

1. Introduction . . . . . . . . . . . . . . . . . 70

2. Literature review . . . . . . . . . . . . . . . . 73

3. Experimental design and procedures . . . . . . . . . 75

3.1 Experimental design . . . . . . . . . . . . . 75

3.2 Experimental procedures . . . . . . . . . 81

4. Results . . . . . . . . . . . . . . . . . . . 81

4.1 Descriptive statistics . . . . . . . . . . 81 
4.2 Testing rationality $\ldots \ldots \ldots$. . . . . . . 83

4.3 Structural model: parameters estimation with the CBSs . 85

4.4 Gender differences . . . . . . . . . . . . . . . 91

5. Discussion and conclusions . . . . . . . . . . . . 93

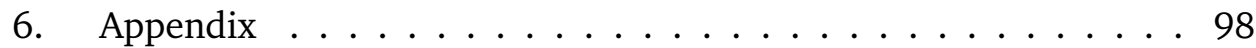

A1 Experimental design details . . . . . . . . . . 98

A2 Structural model additional features and results . . . . 100

A3 Structural estimation by gender . . . . . . . . . . . 104

A4 Individual characteristics and preferences . . . . . . 108

A5 Instructions . . . . . . . . . . . . . . 110

4 The Impact of the Menstrual Cycle on Bargaining Behavior 127

1. Introduction . . . . . . . . . . . . . . . . . . . . . 128

2. Literature review . . . . . . . . . . . . . . . . . . 130

3. Experimental design and procedures . . . . . . . . . 131

3.1 Experimental sessions with bargaining game . . . . . . 132

3.2 Tracking of the menstrual cycle and estimation of cycle

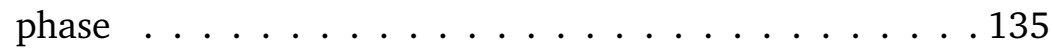

3.3 Experimental procedures . . . . . . . . . . 137

4. Hypotheses . . . . . . . . . . . . . . . . . . . 137

5. Results . . . . . . . . . . . . . . . . . . . 139

5.1 Comparison of informed and uninformed players . . . . 140

5.2 Bargaining behavior: positions and concession rates . . . 141

5.3 Bargaining consequences: Payoffs and deal rates . . . . 148

6. Discussion and conclusions . . . . . . . . . . . . . 153

7. Appendix . . . . . . . . . . . . . . . . . 158

A1 Experimental design details . . . . . . . . . 158

A2 Descriptive statistics . . . . . . . . . . . . . 161

A3 Additional analysis non-pill takers . . . . . . . . . 162

A4 Different length of phases - Non-contraceptive takers . . . 169

A5 Contraceptive takers . . . . . . . . . . . 173

A6 Instructions . . . . . . . . . . . . . 176

5 Conclusion $\quad 187$

6 Impact Chapter 191 


\section{List of Figures}

2.1 Average switching behavior at set level . . . . . . . . . . . . 25

2.2 Percentage of participants per rational switching behavior. . . . . 26

2.3 Distribution of $\omega_{i}$ at the individual level. . . . . . . . . . . 27

2.4 Interquartile distribution of $\omega_{i, n}$-Group size $3 \ldots 29$

2.5 Interquartile distribution of $\omega_{i, n}$-Group size $6 \ldots$. . . . . . . 29

2.6 Intensity of Preferences for Competition, $\omega_{i} \ldots \ldots$. . . . . 30

2.7 Preferences for Competition by treatment, $\omega_{i} \ldots \ldots$. . . . . . 41

2.8 Interquartile ranges distribution of $\omega_{i}$ for treatment $1 \ldots 41$

2.9 Interquartile ranges distribution of $\omega_{i}$ for treatment $2 \ldots$. . . . 41

2.10 Intensity of Preferences for Competition by treatment . . . . . . . 42

2.11 Distribution of $\omega_{i}$ - Whole sample. . . . . . . . . . . . . 43

2.12 Interquartile ranges distribution of $\omega_{i}$ for a group of three -

Whole sample. . . . . . . . . . . . . . . 43

2.13 Interquartile ranges distribution of $\omega_{i}$ for a group of six - Whole sample. . . . . . . . . . . . . . . . 44

2.14 Intensity of Preferences for Competition- Whole sample. . . . . . 44

2.15 Consistency at set level by gender . . . . . . . . . . . . . . 45

2.16 Consistency at the individual level by gender . . . . . . . . . . 45

2.17 Preferences for Competition (in euros) - Distribution of $\omega_{i}$ at the individual level by gender. . . . . . . . . . . . . . 46

2.18 Interquartile ranges distribution of Delta at the individual level by gender. . . . . . . . . . . . . . . . 46

2.19 Intensity of Preferences for Competition by gender . . . . . . 47

2.20 Ranking table . . . . . . . . . . . . . . . . . . 51

2.21 Task 2 - Screen example . . . . . . . . . . . . . . . 52

3.1 Example of a budget line for the choice of the payment-scheme . 77

3.2 Example of a budget line for the choice between the Certain Amount and the Probabilistic Amount. . . . . . . . . . 80

3.3 Distribution of Afriat's (1972) Critical Cost Index (CCEI) for competitive and risky behavior. . . . . . . . . . . . 84 
3.4 PDFs of the estimates reported in Table 3.3 for Model 1. The reference distribution in black is a normal Gaussian. . . . . . . . 90

3.5 PDFs of the estimates reported in Table 3.3 for Model 2. The reference distribution in black is a normal Gaussian. . . . . . . 90

3.6 CCEI for risk preferences . . . . . . . . . . . . . . . . . 91

3.7 CCEI for preferences for competition . . . . . . . . . . 92

3.8 Percentage of participants with an specific number of corner choices in the risk and competition tasks. . . . . . . . . . . . 99

3.9 Estimates of risk aversion $(\alpha)$ and additive preference for competition $(\theta)$ - Model $1 \ldots \ldots$. . . . . . . . . . 101

3.10 Estimates of risk aversion $(\alpha)$ and additive preference for competition $(\theta)$ - Model $2 \ldots \ldots$. . . . . . . . . . . . . .

3.11 Estimates of risk aversion without $(\alpha)$ and with $(\delta)$ competition - Model 2 . . . . . . . . . . . . . . . . . . . 102

3.12 PDFs of the estimates reported in Table 3.3 for Model 1 . . . . . 103

3.13 PDFs of the estimates reported in Table 3.3 for Model 2 . . . . . . 103

3.14 PDFs of the estimates reported in Table 3.4 for Model 1 for Risk preferences without competition $(\alpha)$ by gender . . . . . . . 104

3.15 PDFs of the estimates reported in Table 3.4 for Model 1 for Competitive preferences $(\theta)$ by gender . . . . . . . . . . 104

3.16 Estimates of the coefficient of risk aversion without competition $(\alpha)$ and the coefficient for the additive preference for competition $(\theta)$ by gender . . . . . . . . . . . 105

3.17 PDFs of the estimates reported in Table 3.4 for Model 2 for risk preferences without competition $(\alpha)$ by gender . . . . . . . 105

3.18 PDFs of the estimates reported in Table 3.4 for Model 2 for additive preferences for competition $(\theta)$ by gender . . . . . . 106

3.19 PDFs of the estimates reported in Table 3.4 for Model 2 for risk aversion with competition $(\delta)$ by gender $\ldots \ldots \ldots 6$

3.20 Estimates of the coefficient of risk aversion without competition $(\alpha)$ and the coefficient for the additive preference for competition $(\theta)$ - Model 2 by gender . . . . . . . . . . 107

3.21 Estimates of the coefficient of risk aversion without competition $(\alpha)$ and the coefficient for risk aversion with competition $(\delta)$ Model 2 by gender . . . . . . . . . . . . . . . . . 107

3.22 Summation Task . . . . . . . . . . . . . . . . . 111

3.23 Example Task 3.1 . . . . . . . . . . . . . . . . . . . 113

3.24 Example Task $3.2 \ldots \ldots$. . . . . . . . . . . . . . . . 114 
3.25 Example Task $3.3 \ldots \ldots \ldots \ldots \ldots$. . . . . . . . . . . . . 115

3.26 Example Task $3.4 \ldots \ldots \ldots \ldots$

3.27 Example Task 3.5 . . . . . . . . . . . . . . . . . . . . . . . . 117

3.28 Example Task 4 . . . . . . . . . . . . . . . . . . . . . . . . . 119

3.29 Example Task 5.1 . . . . . . . . . . . . . . . . . . . . . . 120

3.30 Example Task $5.2 \ldots \ldots \ldots$. . . . . . . . . . . . . . . . . . . 122

3.31 Example Task $5.3 \ldots \ldots \ldots$. . . . . . . . . . . . . . . . 123

3.32 Example Task $5.4 \ldots \ldots$. . . . . . . . . . . . . . . . . . . . . . . 124

3.33 Example Task 5.5 . . . . . . . . . . . . . . . . . . . . . 124

4.1 Menstrual phases and hormone levels over the menstrual cycle. . 135

4.2 Bargaining position across time during the simultaneous bargaining stage . . . . . . . . . . . . . . . 141

4.3 Initial offers by informed players. . . . . . . . . . . . . . . . 143

4.4 Initial demands by uninformed players . . . . . . . . . . . . 146

4.5 Payoffs un/conditional on reaching a deal for informed and uninformed players. Notes: The figure shows the payoffs and corresponding standard errors for each menstrual cycle phase. Standard errors are obtained from OLS regressions clustered on the matching group level and controlling for pie size and bargaining round. . . . . . . . . . . . 148

4.6 Electronic menstrual cycle calendar . . . . . . . . . . . . . . . . . 159

\section{List of Tables}

2.1 Example of a decision set $\ldots \ldots \ldots$. . . . . . . . . . 20

2.2 Summary behavior in the experimental tasks . . . . . . . . . 24

2.3 Values for the lotteries in the five decision sets . . . . . . . . . 36

2.4 Probability values for the Individual Pay in Treatment $1 \ldots \ldots 37$

2.5 Descriptive statistics by treatment . . . . . . . . . . . . . . 39

2.6 Competitiveness by treatment . . . . . . . . . . . . . . . . 40

2.7 Average switching behavior at decision set level . . . . . . . . 40

2.8 Average switching behavior at the individual level . . . . . . . 40

3.1 Summary of behavior in the experimental tasks $\ldots \ldots \ldots$. . . 82 
3.2 CCEI for competitive and risky behavior . . . . . . . . . . 85

3.3 Estimated risk and competition preferences at the individual level 89

3.4 Estimated risk and competition preferences by gender . . . . . 993

3.5 Summary of the earnings for each of the parts of the experimental design. . . . . . . . . . . . . . . 98

3.6 Summary of intercepts and corner choices in Parts 3 and 5 of the experimental design. . . . . . . . . . . . . . . . . . 99

3.7 Estimated risk and competition preferences at Population level . . 100

3.8 Estimated risk and competition preferences at the individual level 102

3.9 Relationships between individual characteristics and risk aversion $\alpha_{i}$, additive preferences for competition $\theta_{i}$ and

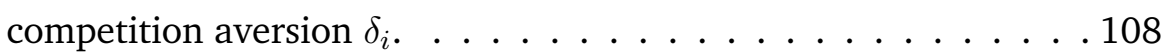

4.1 Lotteries for risk preferences . . . . . . . . . . . . . . . . . 134

4.2 Initial offers and relative concession rates for informed players. . 144

4.3 Initial demands and relative concession rates for uninformed players. . . . . . . . . . . . . . . . . . . 147

4.4 Informed players' unconditional payoffs, payoffs conditional on a deal and deal rates. . . . . . . . . . . . . . . . . . . . . . 149

4.5 Uninformed players' unconditional payoffs, payoffs conditional on a deal and deal rates. . . . . . . . . . . . . . . . . . 152

4.6 Average deals rates for informed and uninformed players . . . . . 153

4.7 Choices for social preferences . . . . . . . . . . . . . . . . 158

4.8 Descriptive statistics . . . . . . . . . . . . . . . . . . 161

4.9 Initial offers and mean relative concession rates for informed players. . . . . . . . . . . . . . . . . . 162

4.10 Initial demands and mean relative concession rates for uninformed players. . . . . . . . . . . . . . . 163

4.11 Informed player's un/conditional payoffs and deal rates with interactions between menstrual phases and risk preferences. . . . 164

4.12 Uninformed player's un/conditional payoffs and deal rates with interactions between menstrual phases and risk preferences. . . . 165

4.13 Mean relative concession rates for both players (Excluding initial offer/demand). . . . . . . . . . . . . . . . . . 166

4.14 Spearman rank-order correlations of Mean relative concession rates for between both players (excluding initial offer/demand) .166

4.15 Risk aversion for both type of players . . . . . . . . . . . . 167

4.16 Benevolence levels for both type of players . . . . . . . . . . 168 
4.17 Initial offers and mean relative concession rates for informed players. . . . . . . . . . . . . . . . . . . . . 169

4.18 Initial demands and mean relative concession rates for uninformed players. . . . . . . . . . . . . . . 170

4.19 Informed players' un/conditinal payoffs and dela rates . . . . . . 171

4.20 Uninformed players' un/conditional payoffs and deal rates . . . . 172

4.21 Initial offer/demand and concession rates for informed and uninformed players. . . . . . . . . . . . . . . . 173

4.22 Informed players' un/conditional payoffs and deal rates . . . . 174 4.23 Uninformed players' un/conditional payoffs and deal rates . . . . 175 4.24 Risk and Social Preferences . . . . . . . . . . . . . . . . . 175 





\section{Chapter 1}

\section{Introduction}

"Of all human powers operating on the affairs of mankind, none is greater than that of competition." Henry Clay

Competition is present in every aspect of our lives and it shapes constantly our relationships and society itself. It ranges from almost unavoidable work experiences such as competing against a co-worker for a wished promotion to more complex situations such as competition between countries for acquiring scarce resources. As a result of our persistent exposure to competition, social and life scientists consider that competitive environments and a certain degree of competitiveness highly influence our future experiences in life and even the evolution of the whole species itself (Darwin's, 1859; Leibbrandt et al., 2013). Despite the relevance of competition in human behavior, there is still an ongoing discussion about what are the determining factors of competitive behavior, and to what extent individuals derive an innate pleasure from a competitive environment or, in other words, whether they have a preference for competition.

A growing body of literature in experimental economics and psychology has recently started to study individuals' preferences for competition closely, especially in the context of gender differences. Using the controlled environment created in a behavioral laboratory, this literature shows that it is possible to capture competitive behavior in isolation from other factors. Since the seminal study of Niederle and Vesterlund (2007), individuals' preferences for competition are captured by looking at how much someone is willing to select into a competitive environment when having an outside option that is not competitive. Interestingly, this measure of preferences for competition seems to capture competitive behavior beyond the lab setting, as it explains differences in the labor market and educational outcomes. For instance, someone's willingness to compete influences pre-university track choices at secondary school level (Buser et al., 2014, 2017a), selection of an ambitious college track in high-school (Almås et al., 2016) or participating in a high-school entry exam (Zhang, 2019). Competitiveness also predicts salary expectations of undergrad students (Reuben et al., 2017), starting salaries and industry choices for MBA students (Reuben et al., 2015), career choices at the vocational level (Buser et al., 2017b), and many more outcomes (Buser et al., 2020).

Several studies in competition show a high degree of heterogeneity in individual preferences for competition, suggesting that competitive behavior is 
dynamic and malleable with the environmental conditions. It has been shown that competition varies across age (Gneezy and Rustichini, 2004; Flory et al., 2018; Mayr et al., 2012), different cultural backgrounds (Gneezy et al., 2009; Andersen et al., 2013; Leibbrandt et al., 2013; Dariel et al., 2017), and gender (Croson and Gneezy, 2009; Niederle and Vesterlund, 2011; Niederle, 2015). In addition, exogenous factors such as stereotype-threat in the task and the group composition (e.g., number of competitors, gender composition and the existence of information contradicting an existing stereotype) can shape competitive behavior (Gneezy et al., 2003; Booth and Nolen, 2009; Geraldes et al., 2011; Iriberri and Rey-Biel, 2012; Dreber et al., 2014; Jung and Vranceanu, 2017).

From a methodological point of view, the regression-based approach is the one commonly used to identify preferences for competition as a determinant of individuals' competitive behavior. With this procedure, the choice between a competitive and a non-competitive environment is attributed to individual's preference for competition, while controlling for the other factors present in a competitive environment. However, recent evidence questioned this approach to capture preferences for competition. In particular, one of the main limitations is that this approach strongly depends on the ability to control successfully for all the other determinants present in a competitive environment (Gillen et al., 2019; van Veldhuizen, 2017). In Chapters 2 and 3 , I propose alternative methods to capture these preferences and I explore whether a preference for competition exists.

But which are the individual characteristics that make someone more or less prone to select into a competitive environment? The literature identifies risk attitudes, beliefs, capability, and more importantly preferences for competition, as central elements in this discussion (for reviews see, e.g., Niederle, 2014; Dariel et al., 2017). Risk preferences seem to be crucial in competitive behavior as most of the competitive environments imply a winner-takes-all situation. That is, setups where only the very best performer rises to the top at the expense of the losers. In this type of situations, a risk averse person might not enter the competition because of the losing scenario, but not necessarily because of the dislike for competition itself. Beliefs also influence competitive behavior as oftentimes people have (over)optimistic views about themselves. This results in an overconfidence bias influencing the decision to enter into a competitive environment. Capability also plays a crucial role as competitive environments usually require some level of ability to perform the task that rules the competition (e.g., sport competitions, product development, exam entry, etc). Lastly, the derived taste (e.g., what economists call preference) or discomfort from taking part in a competition influences someone's decision to select a competitive environment as well.

In addition to these individual traits, biological factors such as the hormones present in the menstrual cycle also influence competitive behavior (Wozniak et al., 2009; Buser, 2012; Ranehill et al., 2018). The evidence of the impact of the menstrual cycle on competitive behavior is somewhat mixed, but overall points towards the important roles of two menstrual phases: the ovulation and premenstruation (or luteal) phases, which are characterized by changing levels of the sex hormones progesterone and estrogen. In particular, the drastic changes of estradiol levels during ovulation-the fertile phase of the menstrual cycle-have been associated with higher levels of risky, competitive, and profit-maximizing behavior; see, for instance, Buser (2012), Wozniak et al. (2009) and Lazzaro et al. (2016). 
The menstrual cycle has also been suggested to influence other economic domains: sealed bid first-price auctions (Chen et al., 2013; Pearson and Schipper, 2013), altruism (Buser, 2011; Ranehill et al., 2018), loss aversion, and economic rationality (Lazzaro et al., 2016). However, the possible link between biological factors and economic decisions has been ignored in the context of bargaining, a type of competitive environment. Chapter 4 delves into the interactions between the menstrual cycle of women and their bargaining behavior.

\section{Overview of the thesis}

To a large extent, the topics in this dissertation discuss questions around competitive and bargaining behavior, which I analyze using behavioral economics and rigorous experimental methods. Besides, I integrate insights from other disciplines such as psychology and biology. In this dissertation, I study the impact of the menstrual cycle on bargaining behavior and whether a preference for competition is a determinant of individuals' willingness to enter tournaments.

The use of laboratory experiments is the common element present in all the studies presented in this dissertation. Differently from field settings, lab experiments allow me to create a controlled environment where I can derive causal links and identify exact mechanisms, something that is hardly achievable with observational data. There are some key features of experiments in behavioral and experimental economics that help to keep control over both the experimenters' and participants' behavior, and that also allow replicability which is at the core of scientific development.

- Randomization. Participants are randomly assigned to the treatment and control groups. This is the golden rule in experiments as it ensures that the two groups do not differ from each other in unobserved or observed dimensions, but only in the exogenous assignment to the treatment. Thanks to this feature, experiments can establish a causal link between the treatment and the observed outcome.

- Monetary financial incentives. Real incentives for individual choices encourage participants to make thoughtful and honest decisions. This ensures that participants reveal their true preferences, and therefore, we can derive predictions from reliable choice behavior.

- No deception. Every information shared with participants is true. This ensures participants trust the instructions and the experimental setting itself, which is crucial to analyze their decision-making processes.

- Anonymity. This removes the observer effect and promotes behavior that will only arise under private circumstances. Also, it ensures careful treatment of the data in line with the guidelines of Ethics Review Committee Inner City faculties at Maastricht University.

One criticism that experimentalists often face concerns the external validity of the behavior and conclusions obtained from a laboratory setting. Specifically, how much a finding can be generalized from an experimental setting to a field scenario? Several studies demonstrate that meaningful insights are derived from experimental settings in different domains such as lying behavior, charitable giving, labor and educational choices, political voting, and many more (Karlan, 2005; Benz and Meier, 2008; Baran et al., 2010; Dohmen et al., 2011; Franzen and Pointner, 2013; Cohn et al., 2015; 
Buser et al., 2014; Herbst and Mas, 2015; Reuben et al., 2015; Potters and Stoop, 2016; Riedl and Smeets, 2017; Fisman et al., 2017; Reuben et al., 2017). Even though I acknowledge that collecting data from a sample that is representative of the entire population would be the best approach, using university students in the lab does not seem to be a major concern. Recent evidence suggests that, for a broad domain of common used experimental outcomes, behavior outside the lab for a representative sample is indistinguishable from undergraduate students' behavior in the lab (Snowberg and Yariv, 2021).

\section{Description of the Chapters}

In Chapter 2, Is there a Preference for Competition? co-authored with Ernesto Reuben, we propose an experimental design that tests whether a preference for competition exists irrespective of the other traits influencing behavior in a competitive environment. Given the relevance of competitive behavior and the possible interaction with other factors, it is crucial to develop an accurate measure of preferences for competition irrespective of other confounding factors. Following the approach by Niederle and Vesterlund (2007), we elicit preferences for competition by the selection between two remuneration schemes for future performance in a real effort task. By adjusting the experimental task we are able to control for individual risk preferences by design, account carefully for beliefs (e.g. how good everyone think they are in terms of relative performance) and generate a rich data set at the individual level which is crucial for testing for consistency and measure preferences for competition irrespective of individual risk attitudes.

Our design offers a number of advantages. First, fixing risk preferences removes the need to statistically control for this trait, which can be potentially contaminated due to measurement error. Second, it allows us to capture a measure of preferences for competition in a non parametric way and directly in our design without the need of using a regression-method approach. Third, we can test consistency of preferences for competition across a wide array of environments, such as competition with different prizes and group sizes.

In Chapter 3, Estimating Preferences for Competition from Convex Budget Sets co-authored with Ernesto Reuben, we continue our investigation on preferences for competition, and study whether preferences for competition can be rationalized by a utility function and develop a structural framework for the joint treatment of preferences for competition and risk. The joint treatment of both preferences allows for an effect of competition preferences on risk preferences, which has been ignored in the classical approach used to measure competitive behavior in the lab. We modify the classical approach of Niederle and Vesterlund (2007) and implement the choice between the two remuneration schemes for future performance as a choice from a convex budget set, similar in spirit to the approach proposed by Andreoni and Miller (2002) to study consistency of choices and individual preferences.

Our design offers a number of benefits for the estimation of preferences for competition. First, it facilitates the test of consistency of individual choices between a competitive and non-competitive environment. Testing for 
consistency is crucial as it provides evidence of a utility-maximizing behaviour, which is necessary to claim the existence of a preference for competition. Second, our design allows us to account for preferences for competition across a wide array of environments, such as competition with different prizes. Third, the rich data set is suitable for estimating preferences at the individual level without the need to aggregate participants together and to assume homogeneity across them.

In Chapter 4, The Impact of the Menstrual Cycle on Bargaining Behavior is based on joint work with Christina Rott and Arno Riedl, we investigate one specific type of competitive behavior for women, bargaining. We present the first study of whether the menstrual cycle of women influences their bargaining behavior and bargaining outcomes. The menstrual cycle is a promising determinant of bargaining behavior as it has been shown to influence women's risk preferences, loss aversion, social preferences, and competitiveness. This literature suggests that women tend to be less competitive, more risk averse and benevolent when the levels of progesterone are high (i.e., during the premenstrual phase) and more competitive and less risk averse during the fertile part of their cycle (i.e., the ovulation phase).

We implement a negotiation environment using a bilateral unstructured bargaining scheme with private information. Following the bargaining game, risk and social preferences are elicited. In addition, before coming to the laboratory, participants are asked to track their menstrual cycle for three months to obtain an accurate measurement of their individual cycle length and menstrual phases. Our findings suggest that bargaining behavior and outcomes vary over the menstrual cycle. We also observe that these differences in bargaining behavior are dependent of the bargaining context, as the information and setting in the negotiation also plays an important role. Understanding the impact of the menstrual cycle on bargaining behavior is important for three reasons. First, the obtained results give insights as to whether nature in the form of the menstrual cycle directly affects negotiation behavior. Second, it will increase our understanding whether risk and social preferences have a mediating effect on bargaining behavior. Third, raising women's awareness of variations in bargaining behavior over the menstrual cycle might influence their bargaining behavior positively, and thereby, help to reduce gender differences in bargaining outcomes on the labor market.

In Chapter 5 I summarize the main findings of this dissertation and in Chapter 6 I briefly describe how my research contributes to the discussion on labor market issues, to policy makers and to society in general. 


\section{Chapter 2}

\section{Is there a Preference for Competition?}

Recent research identified the willingness to compete as an important determinant of individual differences in labor market outcomes like salaries, bonuses and promotions. However, there is no consensus yet as to what are the underlying factors behind competitive behavior. Are participants who are willing to compete more capable, more confident, more tolerant of risk, or are they competing because they enjoy competition per se? This paper contributes to the discussion on preferences for competition and on how to measure them. In this study, we propose an experimental design that tests whether a preference for competition exists by controlling for the role of risk preferences by design and measuring for overconfidence carefully. Our findings provide strong evidence of a preference for competition at the individual level that exists irrespective of risk attitudes. Also, this preference seems to be well defined for most individuals as people are either competition averse or competition seeking and there is small variation of preferences when confronted with different competition stakes. Lastly, our results suggest that preferences for competition are substantial in some cases given that people are willing to pay a significant amount of money for entering or avoiding competition.

This chapter is co-authored with Ernesto Reuben 


\section{Introduction}

In recent decades, economists have started to pay attention to non-cognitive factors as important determinants of economic behavior. After reviewing the economic literature, Heckman et al. (2019) conclude that factors such as psychological traits and preferences explain and cause important life outcomes, like wages and health. More recently, the literature in experimental economics has started to focus on one of these traits, i.e., preferences for competition. Competition and the psychological disposition to select into a competitive environment are present in most aspects of our lives, for instance, the workplace, education, social status, markets, and many more. Besides the relevance of competition in our daily lives, several studies have linked the laboratory measurement of preferences for competition with labor market outcomes and educational choices (Buser et al., 2014; Berge et al., 2015; Reuben et al., 2015; Buser et al., 2017a,b; Reuben et al., 2017; Kamas and Preston, 2018; Zhang, 2019; Buser et al., 2020).

Since the seminal paper by Niederle and Vesterlund (2007), many influential experimental studies have documented individual heterogeneity in preferences for competition (for reviews see, e.g., Niederle, 2014; Dariel et al., 2017). However, it is yet unclear what underlying factors are driving such heterogeneity (Gillen et al., 2019; van Veldhuizen, 2017). Is it due to individual differences in risk attitudes, confidence levels, ability to perform in such an environment, or due to individual differences in a taste for competition? Given the relevance of competitive behavior and the possible interaction with other factors, it is crucial to develop an accurate measure of preferences for competition irrespective of all the other factors present in a competitive environment. We contribute to this discussion by developing an experimental approach that controls for the role of risk preferences by design and accounts for the other factors present in this type of environment. In addition, our method generates a rich data-set that allows us to test with a high degree of confidence whether individual choices are consistent in a competitive environment, and therefore, to test for the existence of a preference for competition.

In the experimental literature, an individual's preference for competition is typically measured using one choice of remuneration schemes when doing a real-effort task. One of the issues associated with this measurement is that it relies entirely on a single individual decision. This could be problematic given that individual choices can be noisy as there is evidence that individuals make different choices when confronted several times with the same set of options (Tversky, 1969; Camerer, 1989; Hey and Orme, 1994; Agranov and Ortoleva, 2017). A second possible limitation is the use of a regression-method approach to measuring preferences for competition, where after controlling for confounding traits such as risk preferences and overconfidence, the residual individual behavior is attributed to the competitive trait. This reduced form approach can bias the measurement of competition considering that it highly depends on accounting properly for the control variables, as it has been extensively discussed in recent literature (Hausman, 2001; Green et al., 2010; 
Westfall and Yarkoni, 2016; Gillen et al., 2019).

This project builds on previous work and improves upon its limitations to test whether a preference for competition exists. Following the approach by Niederle and Vesterlund (2007), we elicit preferences for competition by the selection between two remuneration schemes for future performance in a real effort task. In addition, by adjusting the experimental task we are able to control for individual risk preferences by design and to generate a rich data set at the individual level which is crucial to test for consistency and measure preferences for competition irrespective of individual risk attitudes. In the task, participants choose between individual and competitive pay. The former scheme depends solely on individual performance whereas the latter depends on relative performance. Different from the design proposed by Niederle and Vesterlund (2007), the remuneration under the individual pay is not certain and involves the same risk that the competitive pay has. We obtain participants' belief of winning the competition before the selection of the payment-scheme and include these beliefs in the individual pay. Thus, the probabilities used in the individual pay are around the range of the subjective probability of winning the tournament for the tournament pay. This approach has the advantage to ensure that the decision between the individual and competitive pay are comparable in terms of risk levels and accounts for individual subjective beliefs of winning the tournament at the moment of choosing of the scheme.

Similar to Niederle and Vesterlund (2007), in our experimental design we use the individual choice between the two schemes as our measurement of preferences for competition. However, an additional new feature of our design is that we represent choices between the two payment-schemes as gambles in a multiple price list (MPL) where we vary the stakes under an individual and competitive pay (i.e., prizes and probabilities in both schemes). This has the advantage of generating several choices at the individual level which allows us to measure preferences with a high degree of confidence by looking at the participants switching behavior within a MPL. Also, thanks to this feature of our design we can test the consistency of preferences for competition across a wide array of environments, such as competition with different prizes and group sizes. Varying the group size gives the possibility to test the intuition that increasing the number of competitors decreases competition seeking behavior as the chances of winning the tournament decrease (Che and Gale, 2003; Garcia and Tor, 2009; Boudreau et al., 2011; Hanek et al., 2016). ${ }^{1}$

Another main feature of our methodology is that it controls for the role of risk preferences by design. This feature has a number of advantages. First, fixing risk preferences removes the need to statistically control for this trait, which can be potentially contaminated due to measurement error. Second, it allows us to capture a measure of preferences for competition in a non parametric way and directly in our design without the need of using a

\footnotetext{
${ }^{1}$ We note however that the opposite intuition could also hold: an increasing number of competitors could increase competition seeking behavior as social comparison concerns might play a stronger role. This could increase the anticipated utility of being the winner in a bigger group.
} 
regression-method approach. Hence, we obtain participants' preferences for competition by looking directly at their decisions between the competitive pay and the individual pay in the MPLs. For instance, in our setup, someone who is competition neutral will always switch from one pay to the other when the expected utility of both payment-schemes is equal in the MPL. Someone who is competition averse will always switch from the competitive pay to the individual pay when the expected utility of the former payment-scheme is higher than the one from the later payment-scheme, and the opposite for someone is competition seeking.

Our main findings reveal the following. We find strong evidence of a preference for competition that is highly consistent at the individual level. Specifically, after controlling for risk preferences by design and accounting for individual beliefs and performance, we observe that most participants in our sample switch from one payment-scheme to the other when they are not indifferent between them. This behavior suggests that preferences for competition exist irrespective of risk attitudes. Our findings also suggest that most of the participants have a substantial preference for competition. In fact, $75 \%$ of participants are competition seeking (45\%) or competition averse $(30 \%)$. In addition, these preferences seem to be defined and common for most of the individuals as we observe that after introducing changes in the competition stakes, there is not much variation in the direction of the preferences for competition at the individual level. Our findings also reveal two more interesting patterns. First, competition seeking behavior increases in bigger groups as we observe that $51 \%$ of participants in a group of six people are competition seeking in contrast to a $36 \%$ of participants that are competition seeking in a group of three people. Second, the common finding that men are more competitive seeking than women (Gneezy et al., 2003; Booth and Nolen, 2012; Dariel et al., 2017; Saccardo et al., 2018), seems to vanish when we control for risk preferences by design and confront participants to different competition stakes. This suggests that risk preferences might drive the observed gender difference in competitive environments as recently suggested by Gillen et al. (2019) and van Veldhuizen (2017).

The remainder of this chapter is as follows. Section 2 provides a literature review and Section 3 describes our theoretical framework. We describe the experimental design and procedures in Section 4. In Section 5, we present our results for consistency behavior and measurement of preferences for competition. In Section 6, we discuss our results, and lastly, we conclude in Section 7.

\section{Literature review}

This paper contributes to the discussion on competitive behavior and on how to measure it in the laboratory. Starting with the seminal paper of Niederle and Vesterlund (2007) (henceforth NV), there has been a lot of attempts in trying to capture willingness to compete, especially in the context of gender differences (for reviews see, e.g., Niederle, 2014; Dariel et al., 2017). These studies are based on the NV measure and document individual heterogeneity 
in competitiveness. Specifically for the context of gender differences, this evidence suggests that men are twice as likely as women to select into competition and that these gender differences in selection are partly captured by gender differences in a taste for competition.

In the classical experimental task of $\mathrm{NV}$, an individual's preference for competition is measured by using the choice of a remuneration scheme when doing a real-effort task. Specifically, participants can choose between two different schemes: a piece-rate, that depends only on individual performance; and a tournament rate, that depends on relative performance. The tournament option is considered a competitive scheme as it requires participants to compete against each other. With the help of a regression-based approach and after controlling for individual's overconfidence, risk preferences, and ability, the choice between the two remuneration schemes is considered as individual's preference for competition. ${ }^{2}$

In addition to measure the competitive trait in the lab, there is evidence that a higher willingness to compete positively correlates with labor market outcomes and educational choices. In particular, individual competitiveness seems to explain career choices in secondary (Buser et al., 2014, 2017a,b; Zhang, 2019) and tertiary education (Reuben et al., 2017; Kamas and Preston, 2018), performance of entrepreneurs (Berge et al., 2015), salaries, bonuses, and industry choice (Reuben et al., 2015), and many more (Buser et al., 2020).

Despite the wide use of the lab measurement of preferences for competition and the important role at the moment of explaining educational and labor market choices, there is not yet an agreement on what this lab measure captures. The vast majority of studies rely on a single individual measure and uses a regression-based method, whose accuracy depends strongly on the ability to control successfully for all the other determinants present in a competitive environment. One potential limitation of this approach is that a measurement error or misspecification in any of these determinants can bias the interpretation of the results (Hausman, 2001; Green et al., 2010; Westfall and Yarkoni, 2016; Gillen et al., 2019).

For the specific case of gender differences, two studies have attempted to address preferences for competition with a different approach. First, Gillen et al. (2019) develop a statistical technique to correct for measurement error in the risk attitudes and overconfidence measures used in the NV design. Their findings suggest that the role of the competitive trait in explaining the gender gap in competition disappears, after accounting for the measurement error of these two traits. Second, van Veldhuizen (2017) proposes a modified version of $\mathrm{NV}$ to differentiate by the experimental design between risk attitudes, overconfidence, and preferences for competition. His design introduces treatments that remove sequentially the role of competition and

\footnotetext{
${ }^{2}$ Other factors associated to individual heterogeneity in a competitive environment are differences in ambiguity attitudes and feedback aversion (Wozniak et al., 2016; Ertac and Szentes, 2011; Friedl et al., 2017). In our study, we do not target these factors since they do not take place in our experimental setting or they are inherently present in a competitive environment as in the case of ambiguity.
} 
overconfidence, and compares these treatments with the classical NV outcome. $^{3}$ In contrast to most of the previous studies in competition, his findings suggest that the gender gap in competition is mainly captured by gender differences in risk preferences and overconfidence. As in van Veldhuizen (2017), we also control for risk preferences by design. However, one main difference with his approach is that in our experimental setting competition is always present, given that it is our main trait of interest. Also, differently from van Veldhuizen (2017) we do not rely on one single individual choice, but we capture individual preferences for competition by confronting individuals to a wide range of scenarios generated with the MPL setting. This new feature of our design allows us to test for consistency of preferences for competition.

\section{Theoretical framework}

In this section, we describe the theoretical background we use to capture preferences for competition. We start by discussing the classical framework employed in the literature and then use this framework to develop our measurement of preferences for competition.

In the NV experimental task, participants choose between an Individual Pay and a Competitive Pay for remuneration in a real effort task. Like in our design, the task consists in adding-up sets of four two-digit numbers during four minutes. Under Competitive Pay, participants get a high amount (HA) per each correct sum if they answer the highest number of correct sums in a group of four people, and a low amount (LA) of zero otherwise. Individual Pay pays participants a certain amount (CA) per each correct sum. Given that in Competitive Pay participants are assigned to groups of four, under the Individual Pay the certain amount equals one-quarter of the high amount. ${ }^{4}$

Following this classical framework, several studies show that there are four correlated factors influencing participants' willingness to select one paymentscheme over the other (for reviews, see, e.g., Niederle, 2014). The first one is risk preferences, given that Competitive Pay implies a winner-takes-all situation and not winning the tournament results in earnings equal to zero. The second one is beliefs about the chances of being the winner in the group. Oftentimes, participants have (over)optimistic views about their true performance in the adding task, resulting in an overconfidence bias influencing their decision to take Competitive Pay. The third one is participants' ability levels to perform

\footnotetext{
${ }^{3}$ For a different approach on how to control risk attitudes by design please refer to Geraldes (2020). His approach is different from the NV approach and also to ours. Specifically, when controlling for risk preferences by design he only uses individuals that self-selected into the noncompetitive payment since the beginning of the experiment. In consequence, in Geraldes (2020) preferences for competition are not measured irrespective of the role of risk attitudes as we do in our design.

${ }^{4}$ In the original experimental task of NV, Individual Pay and Competitive Pay are called as piece-rate pay and tournament pay, respectively. What we call in our theoretical framework high amount, low amount, and certain amount corresponds to a payoff in the NV task of $2 \$, 0 \$$ and $0.25 \$$ per correct sum, respectively. We frame the payoffs in this way to make our setting comparable to the NV setting.
} 
well in the adding task. Lastly, a preference for competition can also push someone to select a Competitive Pay if this person derives pleasure (i.e., utility) from a competitive environment.

Accounting for these four factors present in a competitive environment and following the formulation proposed by van Veldhuizen (2017), it is possible to represent the decision for individual $i$ between Individual Pay and Competitive Pay as follows:

\section{Classical framework - NV}

\begin{tabular}{|l|c|}
\hline Individual Pay & Competitive Pay \\
\hline$\pi_{i}^{C A}$ for sure & belief $_{i}$ chance of getting $\pi_{i}^{H}$ \\
\hline
\end{tabular}

where $\pi_{i}^{C A}$ denotes the certain amount in euros for individual $i$ (i.e., $\mathrm{CA} *$ sums $_{i}$, where sums $s_{i}$ represents the number of correct sums individual $i$ has in the adding task), $\pi_{i}^{H}$ is the high amount in euros for individual $i$ (i.e., $\mathrm{HA} * s \mathrm{ums}_{i}$ ) and belief $f_{i}$ is the subjective belief of being the winner in their group. ${ }^{5}$ Following expected utility theory, we assume that there is a utility function $U\left(\pi_{i}, C\right)$ that evaluates payoffs under Competitive Pay, and depends on the monetary payoffs $\pi$ (from the high and low amounts) and the utility derived from competition $C$. In addition, the same utility function evaluates differently the payoffs under the Individual Pay, as competition is not present, i.e., $U\left(\pi_{i}, 0\right)$. Thus, individual $i$ will choose Competitive Pay if:

$$
\text { belie } f_{i} * U\left(\pi_{i}^{H}, C\right)+\left(1-\text { belie }_{i}\right) * U\left(\pi_{i}^{L}, C\right)>U\left(\pi_{i}^{C A}, 0\right) .
$$

Where $\pi_{i}^{L}$ denotes the low amount in euros for individual $i$ or the amount obtained in case of losing the tournament (i.e., LA $* s u m s_{i}$ ). ${ }^{6}$ For this inequality to hold, one of two scenarios needs to happen. On one hand, preferences for competition $C$ are strong enough. Or on the other hand, participants have a level of overconfidence belie $f_{i}$ or risk preferences high enough to compensate for the dislike in competition. As a result, not only preferences for competition are responsible for the choice of Competitive Pay, but also factors such as risk attitudes and overconfidence could influence this decision. Having these three factors together in a competitive environment can raise the concerns suggested by Gillen et al. (2019) and van Veldhuizen (2017). Also, it relies heavily on the assumption that risk and competitive preferences are orthogonal.

In our framework, we remove the confounding effects of risk preferences by design and hold the effect of overconfidence constant, and we are thus able to identify the role of preferences for competition directly from the decision between Competitive Pay and Individual Pay. In particular, when an individual $i$ faces a decision between the two payment-schemes in our experiment, she evaluates the following two options:

\footnotetext{
${ }^{5}$ Note that in the classical framework, under the competitive amount there is a belie $f_{i}$ chance of getting $\pi_{i}^{H}$ and a 1 - belief $f_{i}$ chance of getting $\pi_{i}^{L}$ (where $\pi_{i}^{L}=\mathrm{LA} *$ sums $_{i}$ ).

${ }^{6}$ Note that in the NV design the low amount is equal to zero.
} 


\section{Our framework}

\begin{tabular}{|c|c|}
\hline Individual Pay & Competitive Pay \\
\hline $\operatorname{prob}_{i}^{H}$ chance of getting $\pi^{H}$ & belief $_{i}$ chance of getting $\pi^{H}$ \\
\hline
\end{tabular}

where $\operatorname{prob}_{i}^{H}$ is the probability in the Individual Pay of obtaining the high amount (HA). The key difference with the classical framework NV is that the Individual Pay does not provide a certain amount. In contrast, the Individual Pay is a lottery that offers the same high amount as the Competitive Pay offers in case of being the winner in the group with a probability $\operatorname{prob}_{i}^{H}$ and the same low amount in case of losing with a probability $1-\operatorname{prob}_{i}^{H}$. Having the same monetary payoffs in the lotteries participants face under both payment-schemes ensures that risk preferences do not play a role. Hence, assuming separability between preferences for competition and risk, an individual $i$ chooses Competitive Pay irrespective of her risk preferences if:

belief $_{i} * U\left(\pi_{i}^{H}, C\right)+\left(1-\right.$ belief $\left._{i}\right) * U\left(\pi_{i}^{L}, C\right)>\operatorname{prob}_{i}^{H} * U\left(\pi_{i}^{H}, 0\right)+\left(1-\operatorname{prob}_{i}^{H}\right) * U\left(\pi_{i}^{L}, 0\right)$.

Since we control for risk preferences by design and account carefully for individual beliefs, we ensure that the choice of Competitive Pays depends only on the individual's preferences for competition. ${ }^{7}$ The competitive trait can then be represented by how differently the same payoffs are evaluated between $U(\pi, C)$ and $U(\pi, 0)$ holding constant and at the same level beliefs and objective probabilities. We assume the following utility function:

$$
U\left(\pi_{i}, C\right)=U\left(\pi_{i}, 0\right)+\theta_{i} .
$$

Where $\pi_{i}$ represents the monetary value of the lotteries for either the high or low amounts and $\theta_{i}$ is the parameter that captures the individual $i$ willingness to pay for entering or for leaving competition. ${ }^{8}$ With the help of our experimental setting, we measure non-parametrically the monetary equivalent of $\theta_{i}$, called $\omega_{i}$, by calculating the difference in the expected utility of Competitive Pay and Individual Pay at the switching point in the MPL. Since in our experimental setting the choice between Individual Pay and Competitive Pay takes place in five MPLs with ten choices in each list, $p r o b_{i}^{H}$ is

\footnotetext{
${ }^{7}$ Differently from the classical framework NV, in our experimental setting the low amount is not equal to zero in all the decision sets. This allows us to explore the role of different prize stakes in participants' taste for competition.

${ }^{8}$ For the proposed functional form of $U$, we follow two assumptions used in a well-known class of utility functions in the literature in experimental economics. First, we assume additive separability between $U$ and $\theta$. In our experimental design, $\theta$ is independent of risk preferences and we do not allow for changes in the curvature of $U$ to changes in $\theta$. One could relax this assumption by allowing the parameter of preferences for competition $\theta$ to be present in the curvature itself. We discuss in detail this functional form in Chapter 3. We also assume quasilinearity of preferences between the payoffs achieved in the Competitive and Individual Pay. That is, $U\left(\pi_{i}, C\right)$ is a linear function of $\theta$ and an increasing function of $\pi_{i}$, as $U^{\prime}\left(\pi_{i}, 0\right)>0$.
} 
the switching probability in the Individual Pay for individual $i$ for obtaining the high amount within a MPL. Specifically, the switching probability $\operatorname{prob}_{i}^{H}$ corresponds to the average between the probability of the high amount in the switching row and the probability of the high amount in the row after the individual switched in the MPL. ${ }^{9}$ Given that individuals face several choices, we take the median value of $\omega_{i}$ for each subject as their preference for competition: ${ }^{10}$

$$
\omega_{i}=\left(\operatorname{prob}_{i}^{H}-\text { belief }_{i}\right)\left(U\left(\pi^{H}\right)-U\left(\pi^{L}\right)\right) .
$$

The intuition behind equations (2.3) and (2.4) is that given someone's reported subjective belief, an individual is considered to be competition seeking if she switches to an Individual Pay when her reported belief belie $f_{i}$ is lower than her switching probability $\operatorname{prob}_{i}^{H}$. Similarly, an individual is considered to be competition averse if she switches to an Individual Pay when her reported belief belie $f_{i}$ is higher than her switching probability $\operatorname{prob}_{i}^{H}$. As a result, competition seeking participants have $\omega_{i}$ greater than zero, competition averse participants have $\omega_{i}$ smaller than zero, and competition neutral participants have $\omega_{i}$ equal to zero. ${ }^{11}$

\section{Experimental Design and procedures}

Participants in our study are invited to a lab session to make choices in an incentivized experiment. In the following two subsections, we describe the experimental design and the experimental procedures, respectively.

\subsection{Experimental Design}

We propose a variation of the experimental task developed by Niederle and Vesterlund (2007) to measure preferences for competition and to control for participants' risk preferences by design. Participants perform an adding task under a selected payment-scheme: Individual Pay or Competitive Pay. We use participants' choice of the payment-scheme as our measurement for their taste for competition. To account for individual beliefs at the moment of selecting the payment scheme, we elicit participants' beliefs of the relative performance in the adding task before they select their preferred payment-scheme. We include two treatment variations in our experimental design. As a robustness check of our experimental design, we vary between subjects the timing of the belief elicitation task (i.e., before or after the selection of the payment-scheme). Also, to test for the role of the number of competitors on preferences for competition, we vary within-subjects the group size between three and six people. In the

\footnotetext{
${ }^{9}$ For MPL without a switch, we use the lowest or the highest value of $\operatorname{prob}_{i}^{H}$ depending on whether the individual always stayed in the Individual Pay or in Competitive Pay.

${ }^{10}$ To arrive at Equation 2.4, we assume without loss of generality that $U(\pi, 0)=U(\pi)$. In Appendix A1, we describe the steps to arrive from Equations 2.2 and 2.3 at Equation 2.4.

${ }^{11}$ As described in detail in Section 5 , we only obtain $\omega_{i}$ for participants that have a unique switching point to fully trust our measurement.
} 
following, we describe in detail each of the parts of the experimental design and the different treatment variations.

Each experimental session starts with an unincentivized practice round of the adding task of three minutes and continues with the following four parts. In the first part, we elicit participants' beliefs of being the best performer in the adding task. In the second part, participants have to choose between two different payment-schemes for their future performance. In the third part, they perform an adding task under a selected payment-scheme. Lastly, they complete a demographic questionnaire. The instructions for each part are provided at the beginning of the respective part and can be found in Appendix A6.

Importantly, before the belief elicitation task, participants are familiarized with the adding task because of the practice round. Participants are also informed they will have to choose between the two payment-schemes for their future performance in the adding task. In addition, although participants know their own absolute performance, they are never informed about the performance of others in the adding task. ${ }^{12}$ Relative performance is only revealed at the end of the experiment if the Tournament Rate is selected for payment purposes. ${ }^{13}$

\section{Part 1: Belief elicitation task}

After the practice round in the adding task, participants guess the likelihood of being the winner in their group (i.e., the probability of having the highest amount of correct sums). An important feature of our belief elicitation task is that participants can answer the belief elicitation question by providing the likelihood of being the winner or the percentile ranking of their performance. ${ }^{14}$

\footnotetext{
${ }^{12}$ Note that this feature creates naturally ambiguity in the tournament as in the tournament payment-scheme participants do not know their relative performance. This is the ambiguity that is usually inherently present in a competitive environment and can be solved by providing feedback on relative performance as proposed by Wozniak et al. (2016). Another way in which ambiguity is present in a tournament is by not knowing the number of competitors as recently suggested by Flory et al. (2015), Balafoutas and Sutter (2019) and Gee (2019). In our setting, the number of competitors is always common knowledge.

${ }^{13}$ Although this feature introduces differences in feedback between the two paymentschemes, feedback on relative performance is one of the key differences between a competitive environment and a non-competitive environment. Also, we believe this is not a concern in our design for two reasons. First, participants never received explicit information about feedback being revealed only if the Tournament Rate was selected for payment purposes. Therefore, participants could not anticipate feedback under only the Tournament rate. Second, although participants still could have beliefs on differences in feedback provision between the two schemes, previous evidence suggests that feedback aversion does not play a role in a setting similar to our (Niederle and Vesterlund, 2007).

${ }^{14}$ For the likelihood of being the winner, participants can choose an answer ranging from 0 (meaning they are completely certain that they are not the winner of their group) to 100 (meaning they are completely certain that they are the winner of their group). For the percentile ranking, participants can choose an answer raging from top 100\% (meaning they are completely certain they performed worse than all other participants of the study, i.e., the worst performer) to the top $0 \%$ (meaning they are completely certain they performed better than all other participants in the study, i.e., the best performer). An example of the two sliders can be found
} 
An advantage of this feature is that participants that struggle thinking in terms of probabilities can answer in terms of ranking instead. Also, we provide them a ranking table displaying the likelihood of being the group's winner associated with any possible rank. ${ }^{15}$

The earnings in this first part can be either $€ 0$ or $€ 20$, depending on how close the likelihood the participants choose is to the actual probability of wining the tournament. We incentivize beliefs using a robust binarized scoring rule (BSR) (Karni, 2009). In particular, given a stated likelihood of being the winner belief, the BSR incentive offers a $1-(1-\text { belief } / 100)^{2}$ chance of earning $€ 20$ in case of being the winner, and a $1-(\text { belief } / 100)^{2}$ chance of earning $€ 20$ in case of being one of the losers. To increase the chances of true reporting in beliefs, we provide participants with information concerning the quantitative incentives in the elicitation method. Using an interactive interface participants could see online the expected earnings in euros associated with any selected likelihood or rank. ${ }^{16}$

\section{Part 2: Selection of the payment-scheme}

After the belief elicitation task but before performing the adding task, participants choose how they want to be paid for each correct sum. Specifically, they can decide between Individual Pay and Competitive Pay in 5 different decision sets that are completely independent of each other. Each decision set is a MPL that contains a series of 10 rows of choices where the left-choices correspond to Competitive Pay and the right ones to Individual Pay. Participants need to make a choice in each of the 10 rows of choices for each of the five decision sets, thus each participant makes a total of 50 choices.

Under Competitive Pay, the earnings depend on individual performance and the performance of others in their group. Participants are randomly assigned to different groups of three or six participants, respectively. The order of the groups is counterbalanced within participants. The participant who correctly solves the highest number of sums in their group is the group's winner. In case of any tie, the winner is determined randomly among the tied group members. If the participant is the group's winner, she earns the high amount (HA) per correct sum, otherwise, she earns the low amount (LA) per correct sum. Under Individual Pay, participant's earnings depend on individual performance and chance. That is, they earn the HA per correct sum with some probability $p$ between $0 \%$ and $100 \%$ and the LA per correct sum with some probability $1-p$. In Table 2.1 we display an example of a decision set. ${ }^{17}$

This task has two key features. The first one is that the probabilities in the

in Appendix A6, Figure 2.21. Note that participants answer only one of the two questions, either the likelihood or the ranking, and by doing so the other question was instantaneously answered as well. In other words, for every provided likelihood participants could see online the corresponding ranking, and for every provided ranking they could see online the corresponding likelihood.

${ }^{15}$ The ranking table is displayed in Figure 2.20 in Appendix A6.

${ }^{16}$ In Appendix A6, Figure 2.21 is displayed the actual screens that participants see during the experiment for the belief elicitation task.

${ }^{17}$ Please refer to Appendix A2, Table 2.3 for the HA and LA in all the five decision sets. 
Table 2.1: Example of a decision set

\begin{tabular}{|c|c|c|c|c|c|c|}
\hline & \multicolumn{2}{|c|}{ Competitive Pay } & \multicolumn{4}{|c|}{ Individual Pay } \\
\hline & Win $(€)$ & Lose $(€)$ & Win $(€)$ & $p$ (Win) & Lose(€) & $p$ (Lose) \\
\hline 1. & 4 & 1 & 4 & 0,17 & 1 & 0,83 \\
\hline 2. & 4 & 1 & 4 & 0,20 & 1 & 0,80 \\
\hline 3. & 4 & 1 & 4 & 0,23 & 1 & 0,77 \\
\hline 4. & 4 & 1 & 4 & 0,26 & 1 & 0,74 \\
\hline 5. & 4 & 1 & 4 & 0,29 & 1 & 0,71 \\
\hline 6. & 4 & 1 & 4 & 0,32 & 1 & 0,68 \\
\hline 7. & 4 & 1 & 4 & 0,35 & 1 & 0,65 \\
\hline 8. & 4 & 1 & 4 & 0,38 & 1 & 0,62 \\
\hline 9. & 4 & 1 & 4 & 0,41 & 1 & 0,59 \\
\hline 10. & 4 & 1 & 4 & 0,44 & 1 & 0,56 \\
\hline
\end{tabular}

Note: This is an example of one decision set with a high amount of $€ 4$ and a low amount of $€ 1$, and a subjective individual belief of $35 \%$.

Individual Pay are estimated at the individual level using as a reference the subjective belief obtained from the belief elicitation task. Specifically, for each decision set, we randomize the position of the subjective belief, and decrease (for the lower rows) or increase (for the higher rows) monotonically the subjective belief in equally spaced steps. ${ }^{18}$ An advantage of this feature is that when participants face a decision between Competitive Pay and Individual Pay in each decision set, the probability of getting the HA under the Individual Pay is around the range of the reported belief for being the winner in their group. In this way, we account for individual beliefs of being the winner at the moment of choosing between the two payment-schemes. The second feature is that the Competitive Pay and the Individual Pay have always the same level of risk within a decision set. Hence, the HA and LA are always the same in all 10 rows for both payment schemes. The only aspect that varies from row to row is the probability of getting the high and low amount under the Individual Pay.

These two features of our design ensure that we control for risk preferences by design and account for beliefs at the moment that participants choose between the Competitive Pay and the Individual Pay in each row. Thus, the difference in the expected utility of Competitive Pay and Individual Pay at the switching point in each decision set captures participants' preferences for competition. For instance, a participant is considered to be competition seeking if she switches to the Individual Pay when her belief of winning the tournament is lower than the probability of winning the HA under the Individual Pay. Similarly, she is competition averse if she switches to the Individual Pay when her belief of winning the tournament is higher than the probability of winning the HA under the Individual Pay.

\footnotetext{
${ }^{18}$ Note that we randomize the position of the beliefs in the MPL from two rows above or below the fifth row. In that way, we avoid that the beliefs are placed in one of the corners of the list. In Appendix A2, we describe in detail how the probabilities in the Individual Pay are calculated using as a reference the subjective belief.
} 


\section{Part 3: Performance under selected payment-scheme}

After the selection of the payment-scheme in the five MPLs, participants perform the adding task knowing the payment-scheme and the specific rate under which they will be paid for each answer in the task. ${ }^{19}$ The adding task consists in adding-up sets of four two-digit numbers during four minutes. The numbers are randomly drawn by the computer from a uniform distribution with a support of 1 to 100. Participants are not allowed to use a calculator, but they are provided a scratch paper. Every time they submit an answer, the computer immediately informs them whether the answer is correct or incorrect and a new sum is generated. In addition, the computer keeps a record of the number of correct sums participants have. Importantly, although participants know their absolute performance, they are never informed about the performance of others in this task.

\section{Part 4: Demographic questionnaire}

As a final step, we ask all participants to complete a demographic questionnaire about characteristics such as gender, age, number of siblings and position among them, nationality, and level of education. ${ }^{20}$ In addition, we add three unincentivized survey questions to elicit general competitiveness on a 7-point scale (Buser et al., 2020; Fallucchi et al., 2020). ${ }^{21}$

\section{Treatment variations}

We use a $2 \times 2$ design, where between-subjects we vary the timing of the belief elicitation task, and within-subjects we vary the size of the groups for the Competitive Pay. The reason behind the first treatment variation is that eliciting beliefs before the selection of the payment scheme as we described in Part 2 could raise concerns of hedging effects. Therefore, we include an additional treatment where beliefs are elicited after the selection of the payment-scheme as a robustness check to account for this concern. ${ }^{22}$ Since we need participants' reported belief to estimate the probabilities displayed in the Individual Pay, for this treatment we obtain the probabilities of the LA and HA differently. Specifically, two additional decision sets are added at the beginning of Part 2 to estimate participants' subjective probability of being the winner with the following 4 steps. First, we confront participants with an

\footnotetext{
${ }^{19}$ After participants choose from all 5 decision sets, one of them is randomly selected. Within the selected decision situation, one of the 10 rows is randomly chosen as well. The type of payment the participants chose in the selected row is used to determine how much they will receive per correct sum in the adding task.

${ }^{20}$ We include controls for the number of siblings as some evidence suggests that having an older sister is positively associated with women's preferences for competition and negatively associated with men's preferences for competition (Okudaira et al., 2015).

${ }^{21}$ The survey questions used to measure general competitiveness can be found in Appendix A3.

${ }^{22}$ Note that this second treatment has mainly a robustness check purposes, as we do not expect preferences for competition to change with the order of belief elicitation task.
} 
initial decision set to understand their direct preferences between the Competitive and the Individual Pay with their switching behavior in this initial decision set. Second, we use participants' choices in this first decision set, and more specifically the number of times they choose the Competitive Pay, to estimate the probabilities in the Individual Pay for the second additional decision set. Third, we use the probability in the switching row and the number of times participants chose Competitive Pay in the second additional decision set to derive their final prediction. Forth, with the final prediction, we estimate the probabilities of the Individual Pay for the final five decision sets in the same way we did in the other treatment. ${ }^{23}$

We also vary the group size between three and six members within-subjects to analyze whether participants are more or less willing to compete in larger groups, and therefore, test for the role of the number of competitors on shaping individual preferences for competition. Due to this within treatment variation, all participants have to answer the belief elicitation question and select the payment scheme twice, one time for a group size of three people and another time for a group size of six people. Lastly, the order of the group size is counterbalanced at experimental session level. ${ }^{24}$

\subsection{Experimental procedures}

The study was conducted at the Behavioral and Experimental Economics Laboratory (BEElab) at Maastricht University. The experiment consisted of 11 sessions of 22 participants on average. We recruited in total 224 participants, 133 women and 91 men, through the online recruitment system ORSEE (Greiner, 2015).

All participants signed an informed consent before participating in the study. They received $\mathrm{a} € 5$ show-up fee and the total earnings were on average of $€ 25$. One of the tasks was selected for payment purposes at the end of the experiment and this was known by the participants from the beginning. The experiment was programmed and executed with the software z-Tree (Fischbacher, 2007).

\section{Results}

To study whether a preference for competition exists, we first check whether individual choices between a competitive and a non-competitive environment are consistent in terms of switching behavior. Once we confirm that competitive behavior is consistent in our experimental setting, we test whether such choices can be captured non-parametrically by a preference for competition with the location of the switching point in each decision set.

This section is divided into four parts. First, we provide descriptive statistics

\footnotetext{
${ }^{23}$ In Appendix A2, we describe in detail the four steps and how the probabilities are estimated in this second treatment.

${ }^{24}$ Note that given the sample size of each experimental session (between 12 and 24 participants) the chances of a participant being matched with the same person twice are larger than zero. However, participants never received feedback on their relative performance in the group. Also, anonymity was always ensured during the whole experimental session.
} 
of our sample; second, we present a consistency analysis in terms of switching behavior in the MPLs for the selection of the payment-scheme; third, we show the results for the measurement of participants' preferences for competition and its variation at the individual level. Lastly, to link our findings with the literature on gender differences in competition, we test for gender differences in both our measurements of consistency and preferences for competition. For the whole analysis, we separate our sample by the group size in the competition (i.e., by groups of three or six people). Since we do not observe significant differences in demographic characteristics or behavior between the two belief treatments (i.e., belief elicitation before or after the selection of the payment scheme), we pool the data from both treatments for the whole analysis. ${ }^{25}$

\subsection{Descriptive statistics}

Table 2.2 displays a summary of the average amount of correct sums in the adding task and the average reported belief of being the winner in a group of three and six people. Participants solve on average 11 sums correctly in both groups and report an average belief of being the winner of 53\% in a group of three and $41 \%$ in a group of six people. A two-sample Wilcoxon ranksum (Mann-Whitney) non-parametric test reveals the following patterns for the different means displayed in Table 2.2. There are no differences in performance conditional of the group size $(p=0.792)$, but significant differences in the reported beliefs between the two group sizes $(p<0.01)$. This finding suggests that people believe to have lower chances of winning the tournament in a group of six people than in a group of three. In addition, we observe that there are significant differences in performance between men and women in a group of three and six people (two-sample Wilcoxon ranksum Mann-Whitney test, $p=0.051$ and $p=0.027$, respectively). The non-parametric test also shows that there are significant differences in beliefs between men and women only in a group of six people but not in a group of three people ( $p=0.046$ and $p=0.307$, respectively).

\subsection{Switching behavior}

In this subsection, we conduct a consistency analysis for the switching behavior during the choice of payment-scheme for future performance. Specifically, our measurement of consistency has two main dimensions. Since we did not impose a single switch restriction in our setting, the first dimension captures the number of times participants switch within a decision set. We

\footnotetext{
${ }^{25}$ The results from Table 2.5 in Appendix A3 suggest that the demographic characteristics such as gender, age, field of studies, nationality and amount of siblings are not statistically different between the two treatments. Using a two-sample test with equal variances, we do not observe any significant difference between treatments in any of these characteristics (all $p$-values are above 0.5). In addition, when comparing the competitive behavior between treatments with three survey questions about competitiveness, we also do not observe significant differences between the answers provided by the participants in each of the treatments (see Table 2.6). In Appendix A3, we provide a separate analysis for treatments 1 and 2, and test for differences in consistency and preferences for competition between the two belief treatments.
} 
Table 2.2: Summary behavior in the experimental tasks

\begin{tabular}{ccccc}
\hline & Group size & $\begin{array}{c}\text { Women } \\
(\mathrm{n}=133)\end{array}$ & $\begin{array}{c}\text { Men } \\
(\mathrm{n}=91)\end{array}$ & $\begin{array}{c}\text { All } \\
(\mathrm{n}=224)\end{array}$ \\
\hline Adding Task & 3 & 10.87 & 12.27 & 11.44 \\
performance & 6 & 10.72 & 12.07 & 11.27 \\
& Average & 10.80 & 12.17 & 11.35 \\
\hline $\begin{array}{c}\text { Average } \\
\text { reported belief }\end{array}$ & 3 & 52.49 & 54.80 & 53.43 \\
of being the winner & Average & 37.90 & 44.72 & 40.67 \\
\hline
\end{tabular}

Note: values represent the average performance in the adding task or average reported belief for each category, i.e., different group sizes, gender, and all participants.

consider an individual to be consistent if she switches at most once in a decision set. The second dimension is about the direction of the unique switch, which we define as rational switch. That is, a switch from the payment-scheme with the lowest expected value to the one with highest expected value, and not the other way around (i.e., from Competitive Pay to Individual Pay). Similarly, an irrational switch is one that happens from Individual Pay to Competitive Pay. Importantly, we are not only interested in participants that have at most one rational switch in all five decision sets, but we also want to check that most of the rational switching behavior is not driven by no-switching behavior in most of the decision sets. For this purpose, we present a consistency analysis both at the individual level, but also at the decision set level.

Figure 2.1 displays the average switching behavior at the decision set level for the two different group sizes. That is, the percentage of decision sets for each of the four possible switching behavior (i.e., rational switch, no switch, multiple switch, and irrational switch). Regarding the participants competing in a group of three players, we observe that around 19\% of the decision sets have zero switching points (i.e., participants stay in either Individual Pay or Competitive Pay all the time), $2 \%$ have multiple switching points (i.e., participants switch multiple times from Individual Pay to competitive or the other way around), and $77 \%$ of the decision sets have one rational switch (i.e., from the Competitive Pay to the Individual Pay). Lastly, around 1.9\% of the sets have an irrational switch (i.e., they switch from Individual Pay to Competitive Pay). We observe a similar pattern for participants competing in a group of six players. That is, around $19 \%$ of the decision sets have zero switching points, $2.5 \%$ have multiple switching points, $76 \%$ of the decision sets have a rational switch, and $2 \%$ of the sets have an irrational switch.

From Figure 2.1, we can conclude that for both group sizes around 95.5\% of the decision sets have either no switch at all (i.e., participants remain always under the same payment scheme) or they have a rational switch. Using a twosample t-test with equal variances, we do not observe any significant difference 


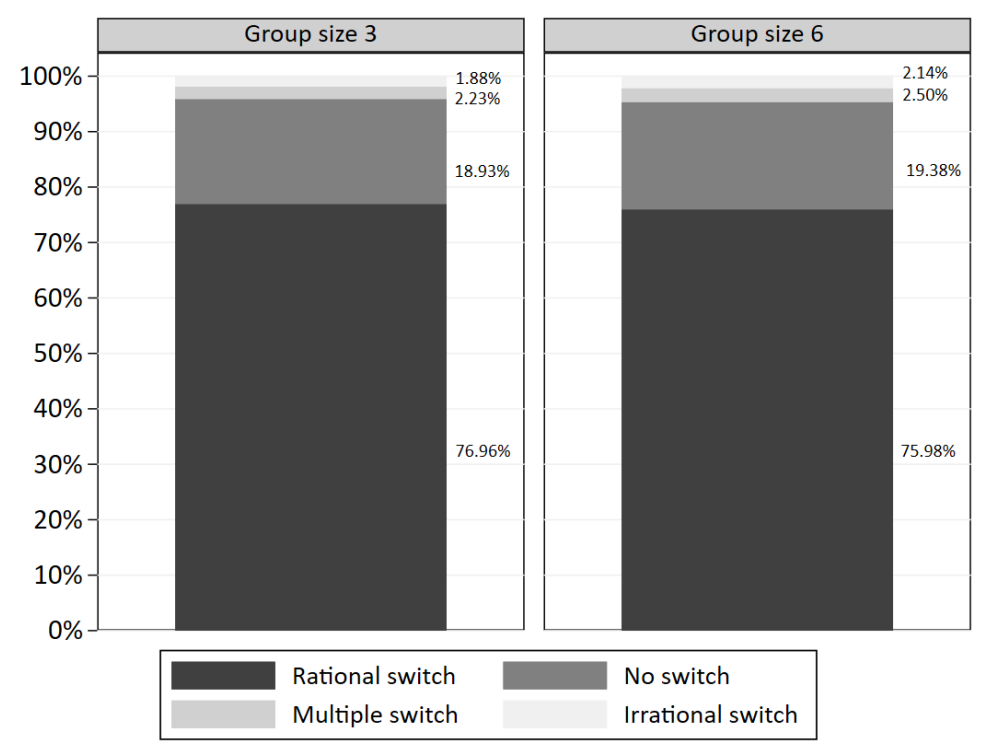

Figure 2.1: Average switching behavior at set level

between group's sizes for the percentage of sets with an irrational switch versus the sets without an irrational switch $(p=0.651)$.

Figure 2.2 shows the percentage of participants with a rational switch in all five decision sets, in three or four decision sets (i.e., majority), in less than two decision sets (i.e., minority), or with one or more decision sets with irrational switch. ${ }^{26}$ For participants in a group of three people, we observe that $54 \%$ participants switch once in all five sets, $21 \%$ switch once in three or four sets, $15 \%$ switch in one or two sets, and only a $9 \%$ have one or more irrational switch. Similarly, for participants in a group of six people, $46 \%$ participants switch once in all five sets, $29 \%$ switch once in three or four sets, $13 \%$ switch in one or two sets, and only a $12 \%$ have one or more irrational switch. Using a two-sample t-test with equal variances, we do not observe any significant difference between group's sizes for the percentage of participants with an irrational switch versus the sets without an irrational switch $(p=0.351)$.

The results from Figure 2.2 suggest overall that $91 \%$ of the participants in a group of three people and $88 \%$ of the participants in a group of six people of our sample have a rational switch or never switched in all five decision sets. ${ }^{27}$ However, since no-switching behavior is problematic for measuring preferences for competition, for the rest of our analysis we keep only participants with a unique rational switch in the majority of decision sets (i.e., three or more sets) and no irrational switch in the other sets. That is, $76 \%$ of the participants in a group size of three and $75 \%$ of the participants in a group of six (170

\footnotetext{
${ }^{26}$ Please note that in the second two categories of Figure 2.2, switched in a majority of sets and switched in a minority of sets, the sets in which participants did not switch refer to sets where there was no-switch at all as these categories are only for participants with no irrational switch.

${ }^{27}$ To obtain the percentage of participants that have a rational switch or never switched, we subtract to $100 \%$ the value of irrational switch displayed in Figure 2.2 for each group size.
} 


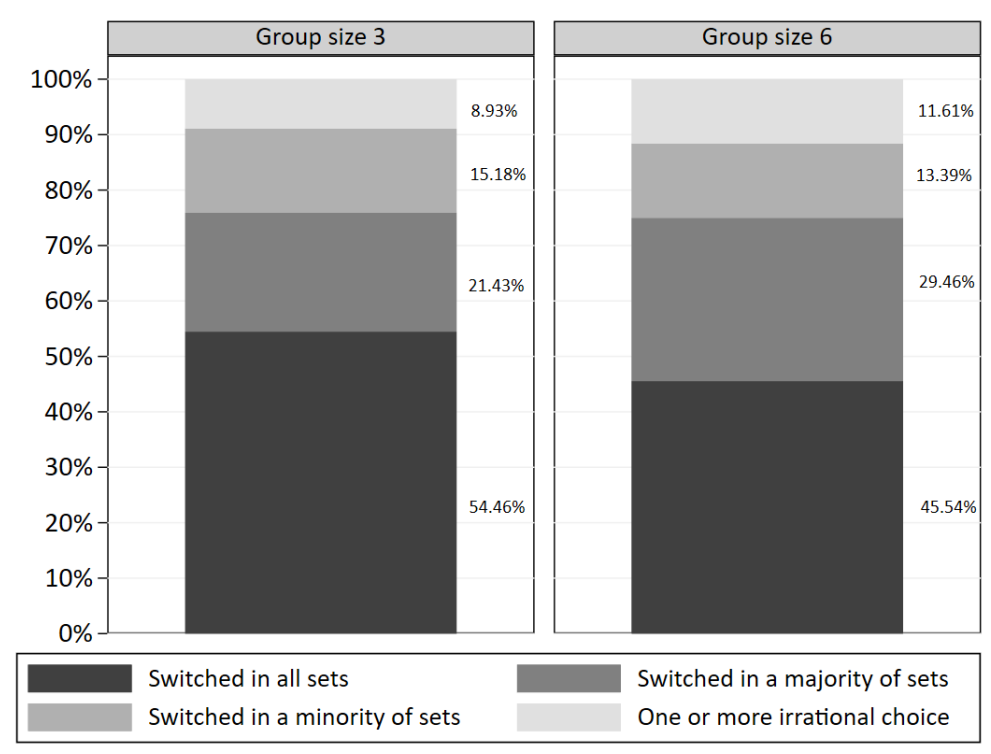

Figure 2.2: Percentage of participants per rational switching behavior.

participants out of 224 for a group size of three and 168 participants out 224 for a group size of six). ${ }^{28}$

Summarizing the results from Figures 2.1 and 2.2, we conclude that competitive behavior is highly consistent and rational at the individual and set level for both group sizes. Furthermore, and even more relevant for measuring preferences for competition, we observe that most of the switching behavior is not driven by participants that did not switch at all from the Competitive Pay to the Individual Pay. ${ }^{29}$

\subsection{Measurement of Preferences for Competition}

In this subsection, we look at the location of the switching point for the participants that have one rational switch in most of the decision sets and do not have any sets with irrational switch (170 participants for a group size of three and 168 participants for a group size of six). We are interested in the values of $\omega_{i}$ obtained from Equation 2.4 at the individual level for a group of three and six people. If participants have a defined competition seeking or competition averse behavior, we should observe an $\omega_{i}$ greater or smaller than zero in most of the decision sets.

\footnotetext{
${ }^{28}$ In the Appendix A4, we present results including also the participants with an irrational switch or the ones who did not switch in more than two decision sets. We do not observe differences when compared to the main results presented in this section.

${ }^{29}$ Tables 2.7 and 2.8 in Appendix A3 display for the two beliefs treatments (i.e., beliefs before and after the selection of the payment-schemes) the average switching behavior at the set and individual level, respectively. A two-sample test with equal variances reveals that there are no significant differences between treatments for the percentage of sets with an irrational switch versus the sets without an irrational switch $(p=0.879)$ or between the percentage of participants with an irrational switch and participants without an irrational switch $(p=0.346)$.
} 
Figure 2.3 displays the distribution of the median preferences for competition at the individual level for the two different group sizes. That is, per individual we obtain the median value of preferences for competition across the five different decision sets for each of the two group sizes. The patterns in Figure 2.3 suggest that at least $65 \%$ and $82 \%$ of the participants have a preference different from zero for a group of three and six, respectively. ${ }^{30}$ In addition, a test for equality of matched pairs reveals that the median subject is slightly competitive in a group of six people $(p<0.001)$, but not in a group of three $(p=0.938)$. Lastly, we also observe that participants are more competitive in larger groups (exact Mann-Whitney ranksum test for equality of medians, $p<0.001) .{ }^{31}$ Suggesting that increasing the number of competitors in the tournament makes participants more competition seeking.

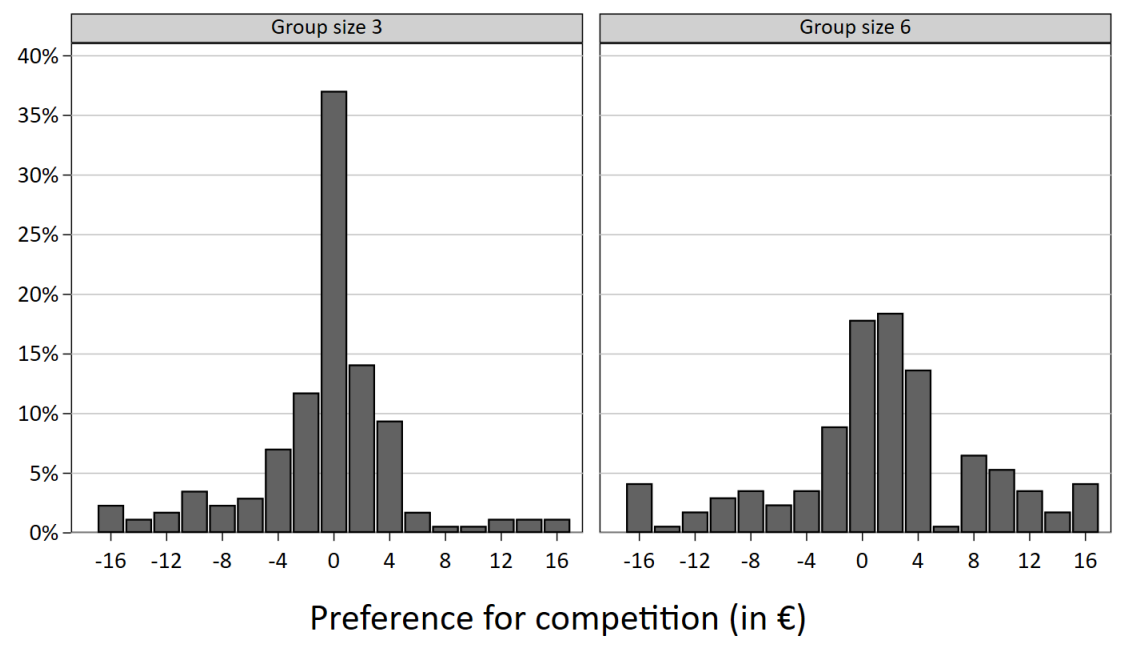

Figure 2.3: Distribution of $\omega_{i}$ at the individual level.

In conclusion, the results from Figure 2.3 suggest that the majority of participants (65\% and $82 \%$, for a group of three and six people respectively) with a unique rational switch in three or more decision sets and no irrational switch in the other sets are either competition averse (i.e., $\omega_{i}<0$ ) or competition seeking (i.e., $\omega_{i}>0$ ). ${ }^{32}$

\footnotetext{
${ }^{30}$ For a group of three people, these are the summary statistics of $\omega_{i}:$ mean $=-0.62$, median $=$ $0.08, \mathrm{sd}=6.08$, min value $=-23.45$ and $\max$ value $=19.71$. For a group of six people, the are the summary statistics of $\omega_{i}$ : mean $=1.71$, median $=2.27, \mathrm{sd}=15.53$, min value $=-60.48$ and $\max$ value $=55.95$. Note that for simplicity, in Figure 2.3 we censored $\omega_{i}$ to values between -16 and 16.

${ }^{31}$ Figure 2.7 in Appendix A3 displays the distribution of the preferences for competition at the individual level for the two different treatments (i.e., beliefs before and after the selection of the payment-schemes). Using an exact Mann-Whitney ranksum test for equality of medians, we observe that there are no significant differences in preferences for competition between treatments $(p=0.514)$.

${ }^{32}$ Note that although a decision error could center the distribution of $\omega_{i}$ to zero as it seems to be displayed in Figure 2.3, we believe this is not a concern in our results for two reasons. First, for a group size of six people, there is a preference for competition significantly different from zero. Second, we observe a small variation of preferences for competition at the individual
} 
Importantly, for obtaining the value of $\omega_{i}$ we aggregate preferences for competition across the five decision sets for each individual. However, to claim the existence of a defined preference for competition we need to check for the variation of such preferences at the individual level. Given that we have more than one measure of preferences per individual, this analysis is possible with our experimental design. We consider a preference for competition to be defined if it has low variation at the individual level and it is the same in most of the decision sets (either competition seeking, averse or neutral in at least three decision sets).

First, we check for the variation of preferences for competition by looking at the variation of $\omega_{i, n}$ within participants and compare it to the variation in $\omega_{i}$ between participants, where $n$ denotes the specific decision set. This analysis helps us to understand whether preferences for competition have a small dispersion, and therefore, capture a defined taste for competition in our sample. If participants do not have a defined preference for competition, we expect to observe a high dispersion of preferences at the individual level. Thus, variation of preference at the individual level should be higher than the variation of preferences between individuals.

Figures 2.4 and 2.5 display the distribution of interquartile ranges of $\omega_{i, n}$ versus the ranges of $\omega_{i}$ which is represented by the vertical line, for a group size of three and six, respectively. ${ }^{33}$ We observe that the interquartile range of $\omega_{i, n}$ for around $76 \%$ of participants in a group of three (see Figure 2.4) and around $67 \%$ in a group of six (see Figure 2.5) is smaller than the interquartile range of $\omega_{i}$. This suggests that most of the participants have a small variation in their preferences for competition, and that for most of the participants, this individual variation is smaller than the variation between participants. Moreover, a two-sample Wilcoxon rank-sum test reveals significant differences in the variance of preferences for competition between the two group sizes $(p<0.001)$. This finding suggests that having a bigger number of competitors increases the variation of preferences for competition. ${ }^{34}$

In short, the information from Figure 2.4 and 2.5 shows that for both groups sizes around $74 \%$ of participants have a small variation in their preferences for competition. As a next step, we describe in detail the observed variation in preferences for competition, and more specifically, the direction or intensity of the preferences for competition at the individual level.

level in both group sizes. In fact, one would expect a high variation of preferences if there is a decision error in the individual choices.

${ }^{33}$ To build the distribution of interquartile ranges displayed in Figures 2.4 and 2.5 we use a measure of statistical dispersion of preferences for competition within and between individuals. Specifically, for each group size we look at the difference between 75 th and 25th percentiles, or between upper and lower quartiles, of individual preferences for competition $\omega_{i, n}$ and compare it with the average difference between both quartiles $\omega_{i}$.

${ }^{34}$ Figures 2.8 and 2.9 in Appendix A3 show the distribution of interquartile ranges of $\omega_{i, n}$ versus the ranges of $\omega_{i}$ which is represented by the vertical line, for each treatment respectively. The patterns in the figures suggest significant differences in the variance of preferences for competition between treatments (two-sample Wilcoxon rank-sum test, $p<0.001$ ) 


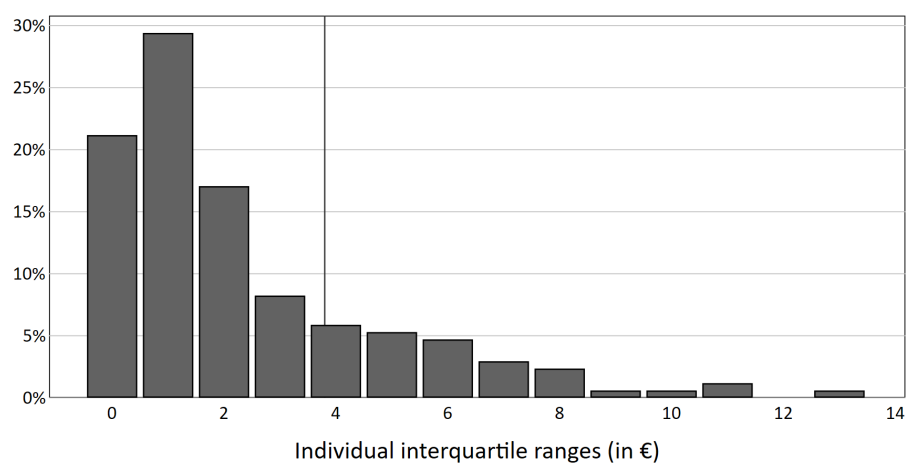

Figure 2.4: Interquartile ranges distribution of $\omega_{i, n}$ within participants and the average interquartile range between participants $\omega_{i}$ (i.e., the vertical line) for a group size of 3 .

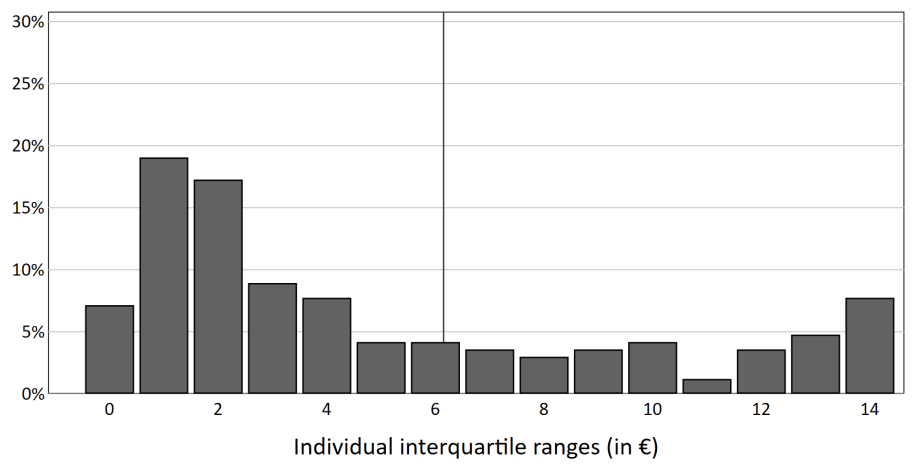

Figure 2.5: Interquartile ranges distribution of $\omega_{i, n}$ within participants and the average interquartile range between participants $\omega_{i}$ (i.e., the vertical line) for a group size of 6 .

In Figure 2.6, we check for the intensity of preferences for competition, or in other words, whether participants have or not the same type of preferences for competition across the different decision sets. To obtain Figure 2.6, we calculate the percentage of participants that have a defined competition seeking $\left(\omega_{i, n}>0\right)$, competition averse $\left(\omega_{i, n}<0\right)$ or competition neutral $\left(\omega_{i, n}=0\right)$ preferences in three or more decision sets among the five sets.

The left panel in Figure 2.6 reveals that for participants competing in a group of three, $73.5 \%$ have a defined preference for competition. That is, a preference that is the same in at least three decision sets. Precisely, $2.4 \%$ participants have a defined competition neutral preference, $36.5 \%$ have a defined competition seeking preference, and $34.6 \%$ have a defined competition averse preference. Finally, $26.4 \%$ of participants do not have a defined preference for competition. The right panel in Figure 2.6 reveals a similar panel for a group of six people as $78.6 \%$ have a defined preference for competition. Specifically, we observe that $4 \%$ participants have a defined competition neutral preference, $51.2 \%$ have a defined competition seeking preference, $23.2 \%$ have a defined competition averse preference and $21.4 \%$ participants do not have a defined preference for competition. Using a two-sample Wilcoxon rank-sum test, we observe significant differences in the intensity of preferences for competition between the two group sizes 


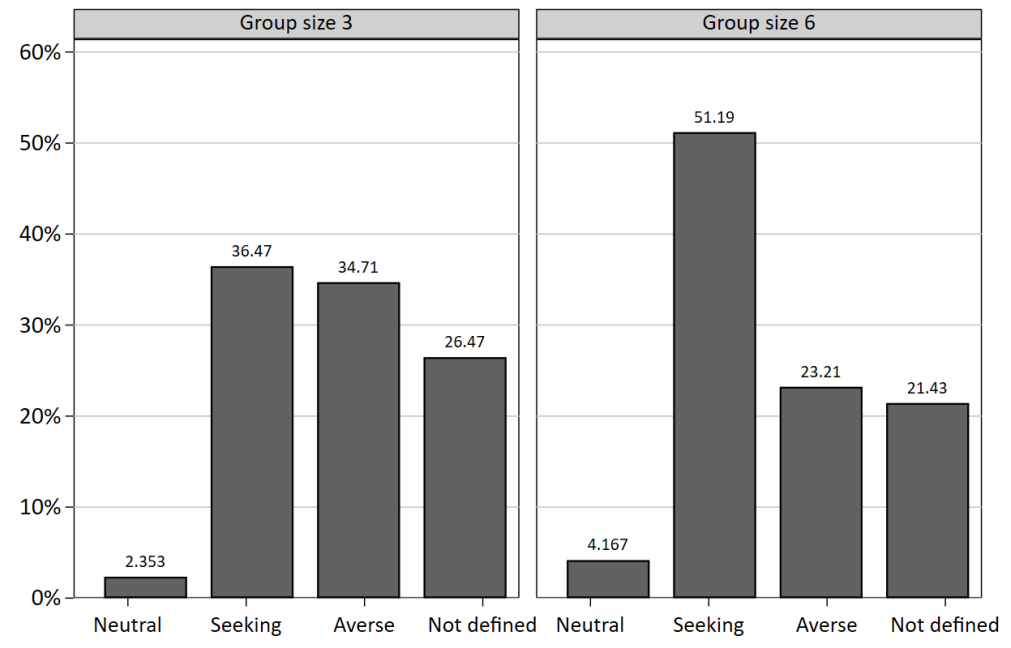

Figure 2.6: Intensity of Preferences for Competition, $\omega_{i}$

$(p<0.001){ }^{35}$ In line with the observed differences in preferences for competition between the two groups sizes in Figure 2.3, Figure 2.6 also shows that participants have more defined competition seeking preferences when they were competing in a group of six people than when they were competing in a group of three.

\subsection{Gender differences}

Since our findings might be informative for the literature in gender differences in competitiveness, we also test whether there are gender differences in the outcomes presented above, both in terms of consistency and preferences for competition. All the respective figures for this section can be found in Appendix A3.

To compare competitive behavior between men and women, we need to ensure first that there are no gender differences in the consistency of the choices they make in a competitive environment. For our two measurements of consistency in switching behavior, our findings do not reveal any significant gender differences. Specifically, there are no significant gender differences between men (mean $=0.982$, sd $=0.004$ ) and women (mean $=0.978$, $s d=0.004)$ for the percentage of sets with an irrational switch versus the sets without it (two-sample test with equal variances, $p=0.4758$ ). Similarly, we do not find significant difference between men (mean $=0.917, s d=0.020)$ and women (mean $=0.883, s d=0.019$ ) for the percentage of participants with an irrational switch versus the sets without an irrational switch (two-sample test with equal variances, $p=0.2435$ ).

For our measurement of preferences for competition, we consider only the

\footnotetext{
${ }^{35}$ Figure 2.9 in Appendix A3 shows the intensity of preferences for competition by treatment. Using a two-sample Wilcoxon rank-sum test, we observe significant differences in the intensity of preferences for competition between treatments $(p<0.001)$.
} 
men and women with a majority of decision sets with a rational switch. For men, we remain with 67 participants (out of 91) for the group size of three and with 69 (out of 91) for a group size of six. For woman, we remain with 103 participants (out of 133) for the group size of three and with 99 (out of 133) for a group size of six. Using a two-sample Wilcoxon ranksum test for equality medians, we do not observe gender differences in preferences for competition (i.e., the $\omega_{i}$ 's values at the individual level for men and women) ( $\left.p=0.203\right)$ nor significant differences in the variance of preferences for competition between men and women $(p=0.107)$. Lastly, using a two-sample Wilcoxon rank-sum test, we also do not find significant differences in the intensity of preferences for competition between men and women $(p=0.135)$. In conclusion, we do not find any evidence of gender differences in preferences for competition or consistency in competitive behavior in our laboratory setting.

\section{Discussion and conclusions}

This study uses an experimental design to measure non-parametrically preferences for competition at the individual level. We modify the experimental task developed by Niederle and Vesterlund (2007) to control for risk preferences by design, account for overconfidence carefully, and generate a rich data set at the individual level. Our results reveal the existence of a highly consistent preference for competition, that can be captured irrespective of risk preferences and is highly defined for most of the participants as it does not vary with changes in the competition stakes or group size.

To test for consistency of individual's choices in competition, we look at the switching behavior during the selection of the payment-scheme. Our results reveal that competitive behavior is highly consistent and rational, and that this is the case for both belief treatments and group sizes. In fact, 95\% of the decision sets contain a rational choice and $89 \%$ of participants make all choices rationally. That is, the majority of sets and participants have at most one single switch between the Competitive and Individual Pay. We also observe that most of these rational choices are not driven by extreme behavior where there is no switch at all (i.e., $76 \%$ of the participants of our sample switched rationally in most of the decision sets). Given that our main goal is to measure preferences for competition, we need to guarantee that individual choices can be interpreted directly from the choices between the two payment-schemes in the different decision sets. We ensure this by calculating preferences for the $76 \%$ of the participants that have a rational switch in most of the sets and taking the individual median preference for competition across the five decision sets.

We capture individual preferences for competition by looking directly at the location of the switch in each decision set. Our findings suggest that most of the participants have a preference for competition, as $75 \%$ of our sample switches when they are not indifferent between the two payment-schemes. In addition, such preferences for competition are well defined at the individual level for most of our sample as $74 \%$ of participants have a small variation in their preferences across the five decision sets. These findings lead us to 
conclude that most of our participants display a defined competition seeking or competition averse preferences. Moreover, connecting this result to our theoretical framework, it suggests that preferences for competition can have an additive shape in the utility participants derive from money in a competitive environment. Although our design only introduces the possibility of an additive shape for these preferences, we believe that this finding can be informative to develop a more accurate measure of preferences for competition. For instance, knowing that there is a fixed taste for competition irrespective of one's risk attitudes, but conditional only on the competition stakes can inform better policymakers when targeting competitive environments. Note that a natural extension to our setting will be to allow for interactions between risk and competition preferences in the utility function. 36

Recently, the existence of the competitive trait has been questioned given the limitations of the most common approach used to measure this trait, see for instance Gillen et al. (2019); van Veldhuizen (2017). However, extensive literature in experimental economics has pointed out the relevance of preferences for competition in explaining individual heterogeneity in a competitive environment, and also differences in educational and labor market outcomes (Buser et al., 2014; Berge et al., 2015; Reuben et al., 2015; Buser et al., 2017a,b; Reuben et al., 2017; Kamas and Preston, 2018; Zhang, 2019; Buser et al., 2020; Fallucchi et al., 2020). Our findings contribute to this discussion as they suggest that even after accounting for risk preferences by design and measuring overconfidence carefully, the trait of competition explains highly individual differences in a competitive environment.

Our results also reveal that $75 \%$ of participants have competition seeking $(45 \%)$ or competition averse preferences $(30 \%)$. This profile of preference suggests a high heterogeneity of preferences for competition. Given that competition is present in every aspect of our lives, for instance in markets, education, and social status, it seems crucial to acknowledge that a high share of the population dislikes competition or do not even have a particular taste for it (around $22 \%$ of our participants do not have a consistent preference for or against competition). Also, a possible venue for future research will be to understand how this individual heterogeneity interacts with other individual traits or cultural aspects of our lives.

Our study suggests that the median participant is competition seeking and that this type of preference is significantly stronger in participants that compete in a group of six in comparison to a group of three people. Moreover, bigger groups also display a higher variance of preferences at the individual level. Interestingly, when looking at differences in beliefs between the two group sizes, we find that participants are less confident of winning the tournament in a group of six than in a group of three people as one would expect. It is intriguing that despite having lower beliefs of winning the

\footnotetext{
${ }^{36}$ I discuss this possibility extensively in Chapter 4 of this dissertation, as neglecting the structural relation between both preferences could lead to biased estimates (Andersen et al., 2008). Interestingly, the findings from Chapter 3 also support the existence of an additive preference for competition.
} 
tournament in a group of six people, participants are more competition seeking in bigger groups than in small ones. Unfortunately, our design cannot bring light on the driving factors of these results, as social comparison and reputation might play an important role when changing the number of competitors and we did not target them in our design. ${ }^{37}$ We believe a more detailed design that allows for changes in social comparison and reputation will be needed to understand the driving differences of preferences for competition with a different number of competitors.

In general, the findings on group size suggest that the number of competitors plays an important role at the moment of shaping individual preferences for competition. There are very few attempts to test the role of the group size on competitors' behavior. Overall, the literature in psychology suggests that competitors' motivation decreases when the number of competitors increases, and that this effect is mediated by social comparison (Garcia and Tor, 2009; Hanek et al., 2016). However, these studies do not account for participants' overconfidence or risk attitudes, which makes it difficult to compare them with our setting. Regarding contests, the literature in economics suggests that increasing the number of competitors decreases the chances of any competitor to win, and therefore, reduces incentives to exert effort (Che and Gale, 2003). In addition, it is also suggested that higher uncertainty reduces the negative effect of added competitors on incentives (Boudreau et al., 2011). In a setting free of uncertainty like ours, we do not observe the same pattern for bigger groups of competitors, given that the level of effort is the same in both group sizes. These opposite findings might highlight the role of uncertainty in a competitive environment. We believe further research is needed to precisely address the role of the number of competitors on preferences for competition, and also to target the role that risk preferences can play in it.

To account for possible hedging effects, we include as a robustness check a treatment variation where we vary the order of the belief elicitation task with respect to the selection of the payment-scheme. We perform a separate analysis for these two treatments in Appendix A3. Our results do not reveal significant differences in the switching behavior or the existence of preferences for competition between these two treatments. However, we observe some puzzling differences in the size of these preferences between these two treatments. Preferences for competition have more extreme values (both negative and positive) in the treatment where beliefs are elicited after the selection the payment-scheme. Also, we observe a higher variance in preferences in the treatment where beliefs are elicited after. Lastly, the intensity of preferences changes between our two treatments. Similar to changes in the group size, these findings reveal that changes in the environment, such as asking beliefs before or after, can shape differently individual preferences for competition. Although it is known that the belief

\footnotetext{
${ }^{37}$ In principle, these factors should not play a role in our design as we did not provide relative feedback to our participants. However, we cannot ensure this as we did not elicit individual beliefs on this matter.
} 
elicitation method can influence participants' actions in a strategic setting (Blanco et al., 2010; Rutström and Wilcox, 2009; Gächter and Renner, 2010), there is not much evidence on the consequences of changing the order of the belief elicitation has on economic behavior and this could be addressed in future research.

An additional point of discussion concerning the belief elicitation task is about the possibility of a biased measure of beliefs due to our elicitation technique. Although we cannot ensure that our belief elicitation method is completely free of measurement error, we consider there are some reasons why we can trust the beliefs provided by participants in our study. First, we use the binarized quadratic scoring rule which offers incentives for truth-telling for a wide range of risk preferences and outperforms other scoring rules and unincentivized elicitations mechanisms (Gächter and Renner, 2010; Wang, 2011; Hossain and Okui, 2013; Harrison and Phillips, 2014; Trautmann and van de Kuilen, 2015). Second, in our setting, there is not an objective prior and it is impossible to test the accuracy of beliefs with respect to a true outcome. An accurate belief of being the winner is not the one that approaches the truth chance of being the winner in the competition, but it is the actual belief that participants use at the moment of choosing whether to compete or not. In consequence, the best we can do in our design is to increase the chances of true telling when it comes to the belief elicitation task. With an incentivized BSR we partly ensure this is the case. Lastly, after the belief elicitation task, we add some noise to the participants' subjective beliefs for the selection of the payment-scheme to construct the probabilities in the Individual Pay. We consider that this noise could help us to account for small deviations participants might have in their beliefs.

Furthermore, recent evidence suggests that providing precise information on the BSR quantitative incentives can alter the truth-telling over an objective prior (Danz et al., 2020). Danz et al. (2020) findings suggest that the false reports in beliefs arise from thinking in terms of probabilities, but it is not entirely clear if this will apply as well to expected earnings. We believe that in our experiment this is not a concern given two features of our belief elicitation task. First, even though we provide details about the quantitative incentives, participants are only informed about the consequences of their selected beliefs in terms of expected earnings and not in terms of the probability of winning the prize. Second, participants have an additional interface that translates every possible likelihood of being the winner in the tournament into terms of ranking (i.e., how one's performance ranks in comparison to the performance of all the other participants). This novel feature of our design helps participants that have problems with thinking in terms of probabilities to understand better the belief elicitation task.

Lastly, we also test for gender differences in consistency and preferences for competition in our experimental setting. Contrary to most of the literature in gender differences in competition (Gneezy et al., 2003; Booth and Nolen, 2012; Dariel et al., 2017; Saccardo et al., 2018), our findings suggest that men and women do not differ in preferences for competition. We show that the common finding that women are less likely to select into a competitive 
environment because they dislike competition more than men (Niederle, 2014), seems to vanish when we control for risk preferences by design. This result is in line with Gillen et al. (2019) and van Veldhuizen (2017) who also observe that gender differences for competition are not captured by the competitive trait. We consider this finding can help to inform policies oriented to increase the representation of women in competitive environments. For instance, targeting other traits such as risk attitudes, overconfidence, and other factors not present in this experiment, could be more effective than targeting the trait of competition itself. 


\section{Appendix}

\section{A1. Theoretical framework - Additional details}

To obtain Equation 2.4 in our theoretical framework, we use the following two equations:

belief $_{i} * U\left(\pi_{i}^{H}, C\right)+\left(1-\right.$ belief $\left._{i}\right) * U\left(\pi_{i}^{L}, C\right)>\operatorname{prob}_{i}^{H} * U\left(\pi_{i}^{H}, 0\right)+\left(1-\operatorname{prob}_{i}^{H}\right) * U\left(\pi_{i}^{L}, 0\right)$,

and

$$
U\left(\pi_{i}, C\right)=U\left(\pi_{i}, 0\right)+\theta_{i} .
$$

In addition, we assume without loss of generality that $U(\pi, 0)=U(\pi)$ ) to obtain the following expression:

$$
\begin{gathered}
\text { belief }_{i} *\left[U\left(\pi_{i}^{H}\right)+\theta_{i}\right]+\left(1-\text { belief }_{i}\right) *\left[U\left(\pi_{i}^{L}\right)+\theta_{i}\right]>\operatorname{prob}_{i}^{H} * U\left(\pi_{i}^{H}\right)+\left(1-\operatorname{prob}_{i}^{H}\right) * U\left(\pi_{i}^{L}\right) \\
\text { belief }_{i} *\left[U\left(\pi_{i}^{H}\right)-U\left(\pi_{i}^{L}\right)\right]+\theta_{i}+U\left(\pi_{i}^{L}\right)>\operatorname{prob}_{i}^{H} *\left[U\left(\pi_{i}^{H}\right)-U\left(\pi_{i}^{L}\right)\right]+U\left(\pi_{i}^{L}\right) \\
\theta_{i}>\left(\operatorname{prob}_{i}^{H}-\text { belief }_{i}\right)\left[U\left(\pi^{H}\right)-U\left(\pi^{L}\right)\right]
\end{gathered}
$$

\section{A2. Experimental design - Additional details}

In this first section of the appendix we provide additional details for our experimental design. Specifically, we provide additional information for the selection of the payment-scheme task and the belief elicitation tasks.

\section{Part 2 - Selection of the payment-scheme}

In Table 2.3, we describe the HA and LA for the five decision sets in Part 2 of the experimental design.

Table 2.3: Values for the lotteries in the five decision sets

\begin{tabular}{ccc}
\hline Decision set & $\begin{array}{c}\text { High amount } \\
\text { (HA in euros) }\end{array}$ & $\begin{array}{c}\text { Low amount } \\
\text { (LA in euros) }\end{array}$ \\
\hline $\mathbf{1}$ & 4 & 0 \\
$\mathbf{2}$ & 6 & 0 \\
$\mathbf{3}$ & 1.5 & 0 \\
$\mathbf{4}$ & 4 & 1 \\
$\mathbf{5}$ & 2 & 0.5 \\
\hline
\end{tabular}

\section{Part 1a - Beliefs before selection of the payment scheme}

In this subsection, we explain in detail how we obtain the probabilities for the Individual Pay from the subjective beliefs participants provide in the belief elicitation task. We refer to the subjective belief as Prediction and use it to estimate the reference probability, i.e., Refprob, used in the Individual Pay. As 
a first step, we take Prediction and add a random number between 2.5 and -2.5 , as displayed below:

$$
\text { Refprob }=\text { round }[\text { Prediction }+(1-2 * \text { random }()) * 2.5,1] ;
$$

Thus, for each decision set $x$, where $x \in[1,5]$, and group size size, where size $\in\{3,6\}$, we ensure the Refprob has a minimum value within the range of the subjective belief, with the following logic formula ${ }^{38}$ :

$$
\begin{aligned}
\text { Refprob }=\min [\text { Refprob, } 92+ & \text { if }(x=2,4,0) \\
\text { if }(x & =3,-4,0) \\
& \text { if }(x=5,-8,0) \\
\text { if }(x & =4 \& \text { size }=6,-4,0) \\
& \text { if }(x=4 \& \text { size }=6,-4,0)]
\end{aligned}
$$

And that for each decision set $x$ and group size size, Refprob has a maximum value within the range of the subjective belief, with the following logic formula:

$$
\begin{aligned}
\text { Refprob }=\max [\text { Refprob, } 10+ & i f(x=2,-5,0) \\
& \text { if }(x=3,5,0) \\
& \text { if }(x==5,10,0) \\
& \text { if }(x=4 \& \text { size }=6,5,0) \\
& \text { if }(x=6 \& \text { size }=6,5,0)]
\end{aligned}
$$

With this value of Reprob, we calculate for each decision set $x$ the probability in the Individual Pay in every row $z$ (i.e., $I P_{x}(z)$ ), with the formulas in Table 2.4, where $z \in[1,10]$.

Table 2.4: Probability values for the Individual Pay in Treatment 1

\begin{tabular}{cc}
\hline Decision set & Probability in the Individual Pay \\
$x$ & $I P_{x}(z)$ \\
\hline $\mathbf{1}$ & $I P_{1}(z)=$ Refprob $+2 *(z-6)$ \\
$\mathbf{2}$ & $I P_{2}(z)=$ Refprob $+1 *(z-6)$ \\
$\mathbf{3}$ & $I P_{3}(z)=$ Refprob $+3 *(z-6)$ \\
$\mathbf{4}$ & $I P_{4}(z)=$ Refprob + if $($ size $==3,2,3) *(z-6)$ \\
$\mathbf{5}$ & $I P_{5}(z)=$ Refprob $+4 *(z-6)$ \\
\hline
\end{tabular}

\footnotetext{
${ }^{38}$ Note that the expression if $(x=a, b, c)$ is interpreted as: if the decision set is equal to $a$, then take the value of $b$, otherwise take the value of $c$.
} 


\section{Part 1b - Beliefs after selection of the payment scheme}

In this second treatment, participants face two additional decision sets to the five decision sets they face in treatment one. In the following steps, we describe in detail how we obtain the probabilities displayed in the Individual Pay in the five decision sets.

1. From the first additional set, we take the number of times participants choose the Competitive Pay and multiply this amount by ten (we call this value Prediction $_{1}$ ). With Prediction 1 , we generate the probabilities for the second additional decision set for each row $z$ in the Individual Pay, where $z \in[1,10]$. Specifically, for each row $z$, the individual probability is calculated with the following logic equation:

$$
I P(z)=30+i f\left(\text { Prediction }_{1}>35,25,0\right)+i f\left(\text { Prediction }_{1}>65,20,0\right)+5 *(z-6)
$$

Equation 2.4 calibrates the obtained value of Prediction $_{1}$ and transforms it into 10 different probabilities $\operatorname{IP}(z)$ for each row $z$ in the Individual Pay. That is, it ensures that for any value of Prediction $_{1}$, the probabilities in $\operatorname{IP}(z)$ do no exceed 100 and are in the range around their predicted belief from the first set.

2. After we obtain the switching behavior of participants in the second additional decision set, we take the number of times participants choose the Competitive Pay and multiply this amount by ten (we call this value Prediction $_{2}$ ). Similar to the first prediction, we calibrate Prediction $_{2}$ with With Equation 2.5:

$$
\text { Prediction }_{2}=2.5+i f\left(\text { Prediction }_{1}>35,25,0\right)+i f\left(\text { Prediction }_{1}>65,20,0\right)
$$

3. With Equation 2.5, we calibrate this prediction with the number of times the individual choose the Competitive Pay in this second set: FinalPrediction $=$ Prediction $_{2}+5 *$ (Number of times Competitive Pay). With this last FinalPrediction, we calculate the reference probability (i.e., Refprob) as we did for treatment 1 (see equations 2.1, 2.2, 2.3, and Table 2.4). 


\section{A3. Analysis separate by treatment}

\section{Descriptive statistics}

To claim that there are no differences among the participants assigned to the treatments where belief are elicited before and after the selection of the payment-scheme, we compare the demographic characteristics of both groups first. Table 2.5 shows a description of different demographic variables in both treatments. We test for differences in the dummy variable Gender that takes the value of one for women; the dummy variable for Economics that takes the value of one if the field of studies is either economics, business or finance and zero otherwise; the dummy variable for Nationality $E U$ that takes the value of one if the nationality is from an European country and zero otherwise. We also account for Age; and the number sisters and brothers individuals grow up with in their household with the variables Sisters and Brothers; and lastly for the position among their siblings.

Table 2.5: Descriptive statistics by treatment

\begin{tabular}{lccc} 
& Treatment 1 & Treatment 2 & \\
\cline { 2 - 3 } Age (mean) & $(\mathrm{n}=107)$ & $(\mathrm{n}=117)$ & $p$-value \\
\cline { 2 - 3 } Gender & 21.17 & 21.15 & 0.938 \\
Economics & $58.87 \%$ & $59.82 \%$ & 0.885 \\
Nationality EU & $69.15 \%$ & $72.64 \%$ & 0.567 \\
\# Sisters & $86.91 \%$ & $87.17 \%$ & 0.953 \\
\# Brothers & 0.71 & 0.65 & 0.624 \\
Position & 0.85 & 0.77 & 0.683 \\
\hline
\end{tabular}

Note: values represent the means for the variables gender, sisters and brothers and position. The p-values correspond to a two-sample test with equal variances.

In addition to the demographic characteristics, we also compare whether there are differences in competitive behavior between the two treatments. For this purpose we use the following three questions: Q1 Competitiveness- I see myself as someone who enjoys winning and hates losing. Q2 CompetitivenessI see myself as someone who enjoys competing, regardless of whether I win or loss. Q1 Competitiveness- I see myself as a competitive person. The answers to these three questions could range from 0 to 6 , where 0 means "completely disagree" and 6 "completely agree". Table 2.6 summarizes the answers to these three questions by treatment.

\section{Results}

In this subsection we present the results for consistency and measurement of preferences for competition separate for the treatments where beliefs are elicit before and after the selection of the payment-scheme.

Table 2.7 displays the average switching behavior at set level for the two different treatments. Similarly, Table 2.8 displays the average switching 
Table 2.6: Competitiveness by treatment

\begin{tabular}{lccc}
\hline & Treatment 1 & Treatment 2 & p-value \\
\cline { 2 - 3 } Q1 Competitiveness & 4.21 & 4.29 & 0.672 \\
Q2 Competitiveness & 3.41 & 3.62 & 0.340 \\
Q3 Competitiveness & 4.21 & 4.22 & 0.970 \\
\hline
\end{tabular}

Note: values represent the means for each of the three questions. The p-values correspond to a two-sample test with equal variances.

behavior at the individual level for the two different treatments. Using a two-sample test with equal variances we do not observe any significant difference between treatments for the percentage of sets with an irrational switch versus the sets without an irrational switch $(p=0.879)$ or the percentage of participants with an irrational switch $(p=0.346)$.

For the results of measurement of preferences for competition, Figure 2.7 shows the distribution of $\omega_{i}$ for both treatment and Figure 2.8 show the intensity of these preferences (i.e., whether they are consistently positive, negative or neutral is three or more decision sets). Lastly, Figure 2.9 and 2.10 show the variance of the preferences for competition at the individual level and compares it with the variation between individuals for both treatments.

Table 2.7: Average switching behavior at decision set level

\begin{tabular}{lcc}
\hline & \multicolumn{2}{c}{ Treatment } \\
\cline { 2 - 3 } & T1: Beliefs before & T2: Beliefs after \\
\hline Rational switch & $68.04 \%$ & $84.19 \%$ \\
No switch & $26.07 \%$ & $12.82 \%$ \\
Multiple switch & $3.83 \%$ & $1.03 \%$ \\
Irrational switch & $2.06 \%$ & $1.97 \%$ \\
\hline
\end{tabular}

Table 2.8: Average switching behavior at the individual level

\begin{tabular}{lcc}
\hline & \multicolumn{2}{c}{ Treatment } \\
\cline { 2 - 3 } & T1: Beliefs before & T2: Beliefs after \\
\hline Switched in all sets & $44.86 \%$ & $54.70 \%$ \\
Switched in a majority of sets & $20.56 \%$ & $29.91 \%$ \\
Switched in a minority of sets & $22.90 \%$ & $6.41 \%$ \\
One or more irrational choice & $11.68 \%$ & $8.97 \%$ \\
\hline Total (n) & 214 & 234
\end{tabular}

Using exact Mann-Whitney ranksum test for equality of medians, we observe that that there are no significant differences in preferences for competition between treatments $(p=0.514)$. 


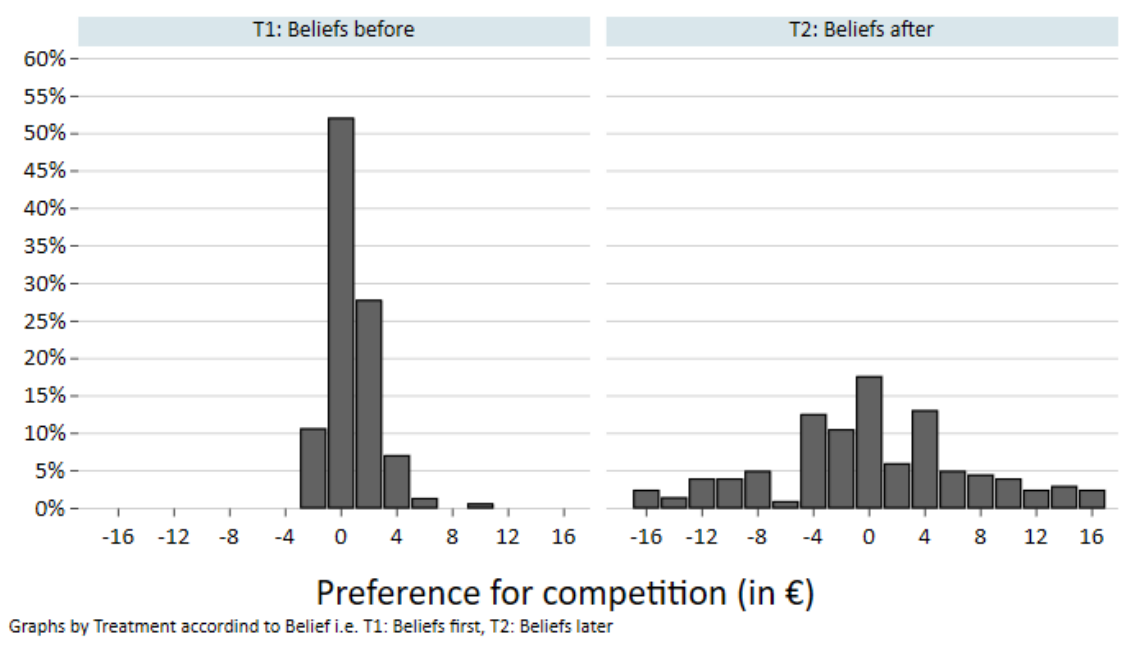

Figure 2.7: Preferences for Competition by treatment, $\omega_{i}$

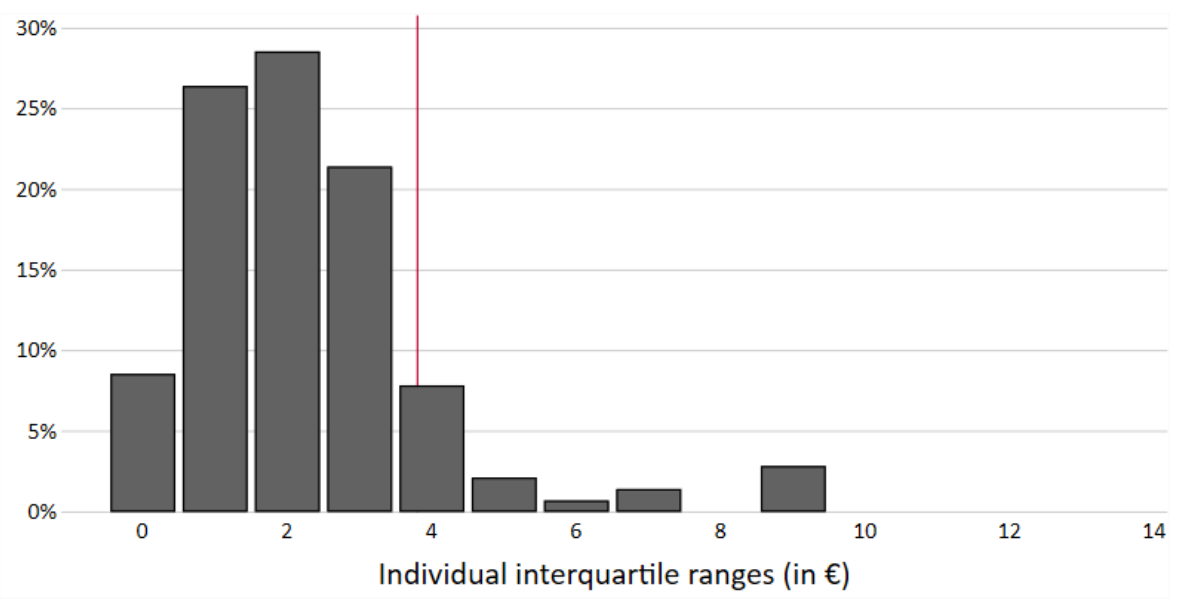

Figure 2.8: Interquartile ranges distribution of $\omega_{i}$ for treatment 1

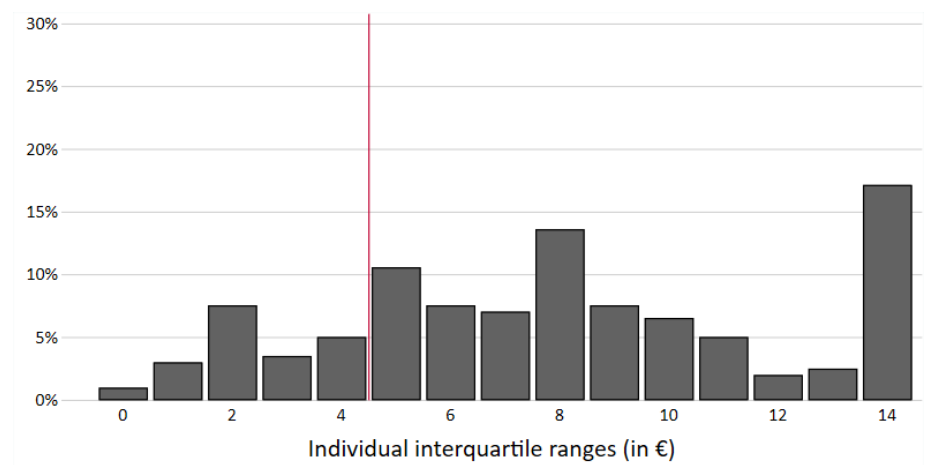

Figure 2.9: Interquartile ranges distribution of $\omega_{i}$ for treatment 2 


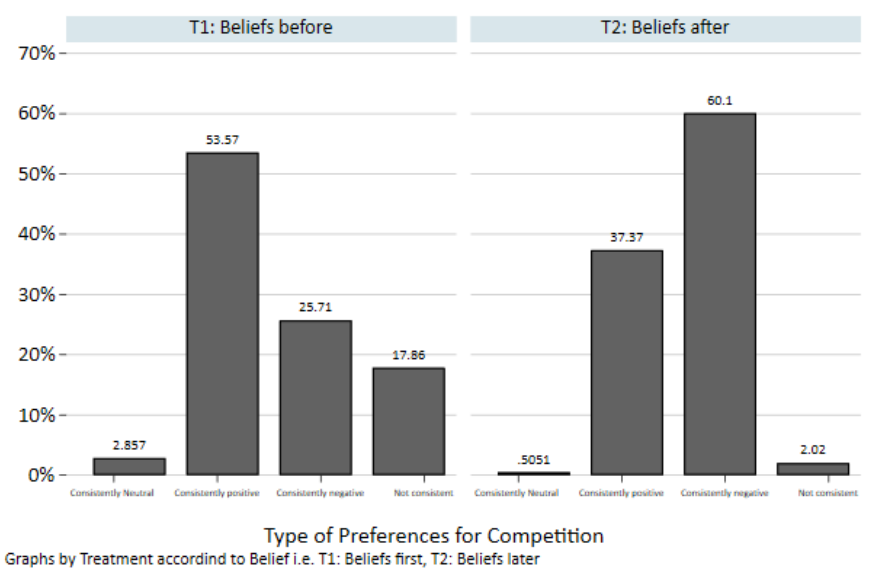

Figure 2.10: Intensity of Preferences for Competition by treatment 


\section{A4. Analysis whole sample - Including inconsistent participants}

In this subsection we present the results measurement of preferences for competition without removing the participants that have inconsistent switching behavior or have less than three decision sets with a rational switch.

Figure 2.11 shows the distribution of $\omega_{i}$ for both group sizes and Figure 2.14 show the intensity of these preferences (i.e., whether they are consistently positive, negative or neutral is three or more decision sets). Lastly, Figure 2.12 and 2.13 show the variance of the preferences for competition at the individual level and compares it with the variation between individuals for both group sizes.

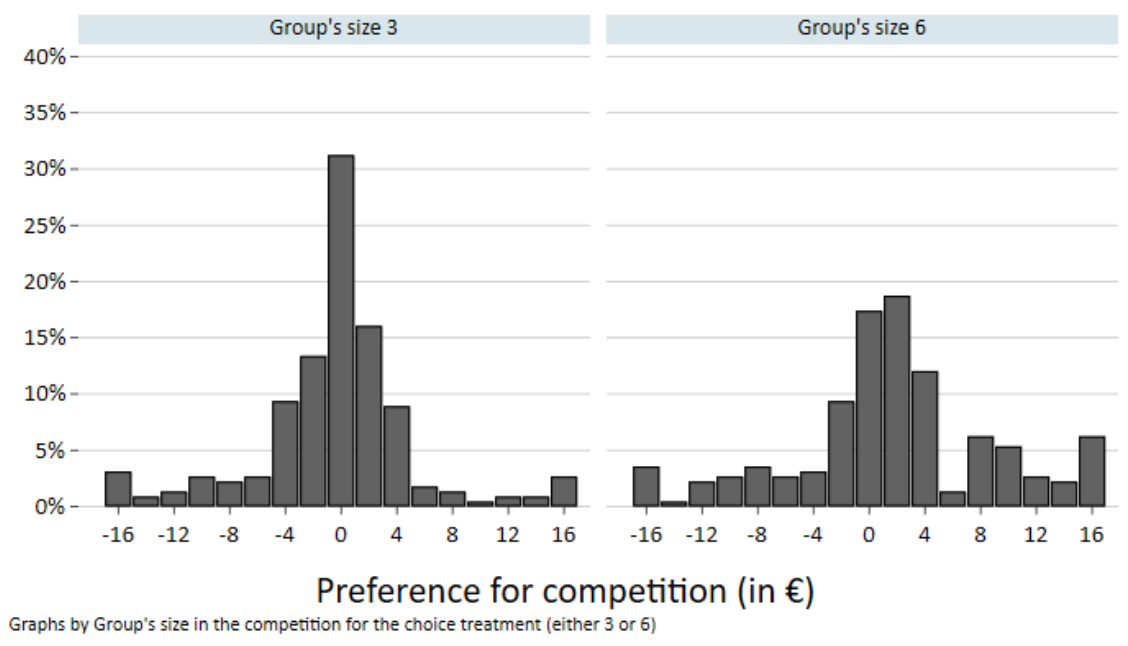

Figure 2.11: Distribution of $\omega_{i}$ - Whole sample.

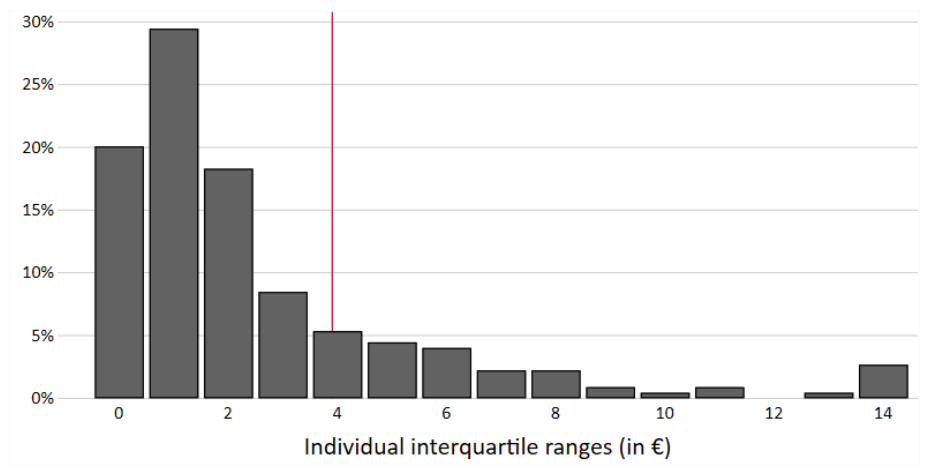

Figure 2.12: Interquartile ranges distribution of $\omega_{i}$ for a group of three - Whole sample. 


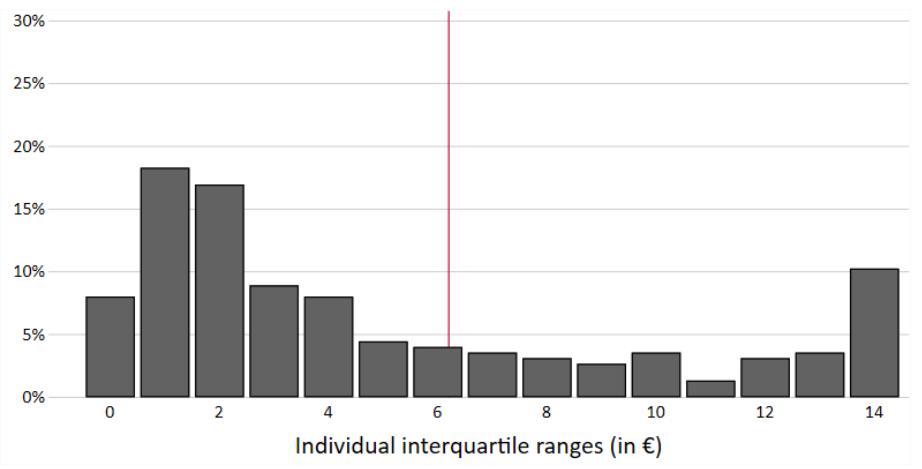

Figure 2.13: Interquartile ranges distribution of $\omega_{i}$ for a group of six - Whole sample.

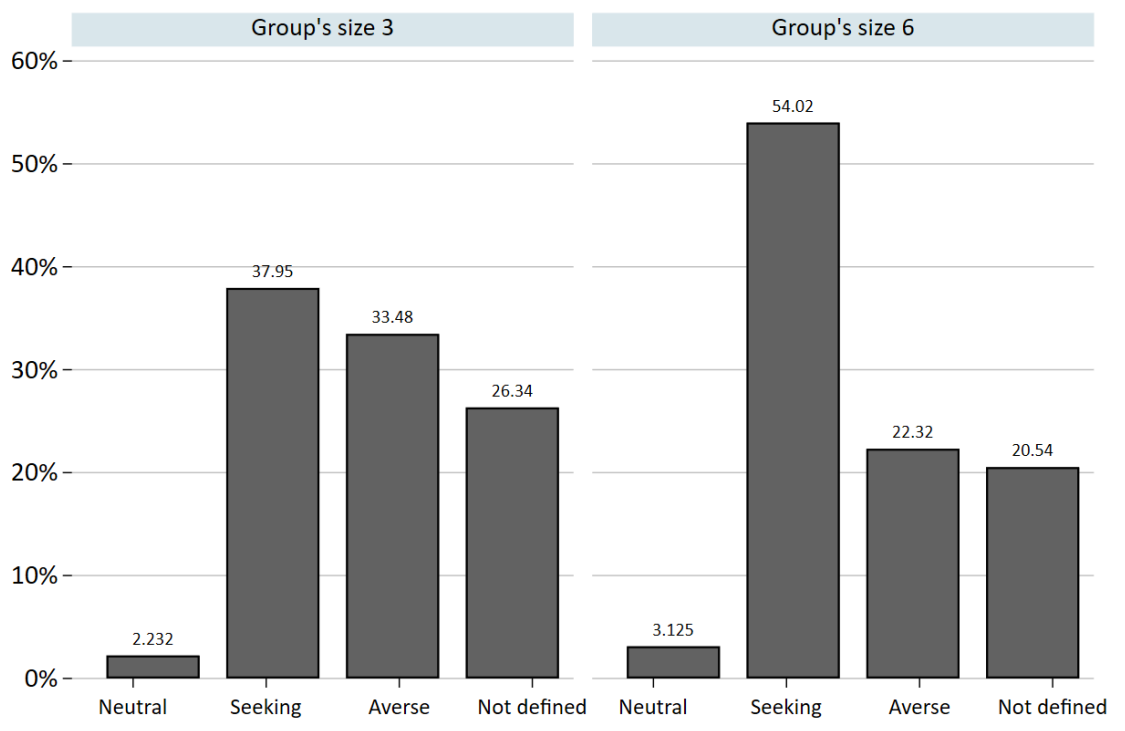

Figure 2.14: Intensity of Preferences for Competition- Whole sample.

\section{A5. Analysis by gender}

In this subsection we present the results for consistency and measurement of preferences for competition separate for men and women.

Figure 2.15 displays the average switching behavior at set level for the two different treatments. Similarly, Figure 2.16 displays the average switching behavior at the individual level for the two different treatments.

For the results of measurement of preferences for competition, Figure 2.17 shows the distribution of $\omega_{i}$ for both treatment and Figure 2.19 shows the intensity of these preferences (i.e., whether they are consistently positive, negative or neutral is three or more decision sets). Lastly, Figure 2.18 shows the variance of the preferences for competition at the individual level and compares it with the variation between individuals for both treatments. 


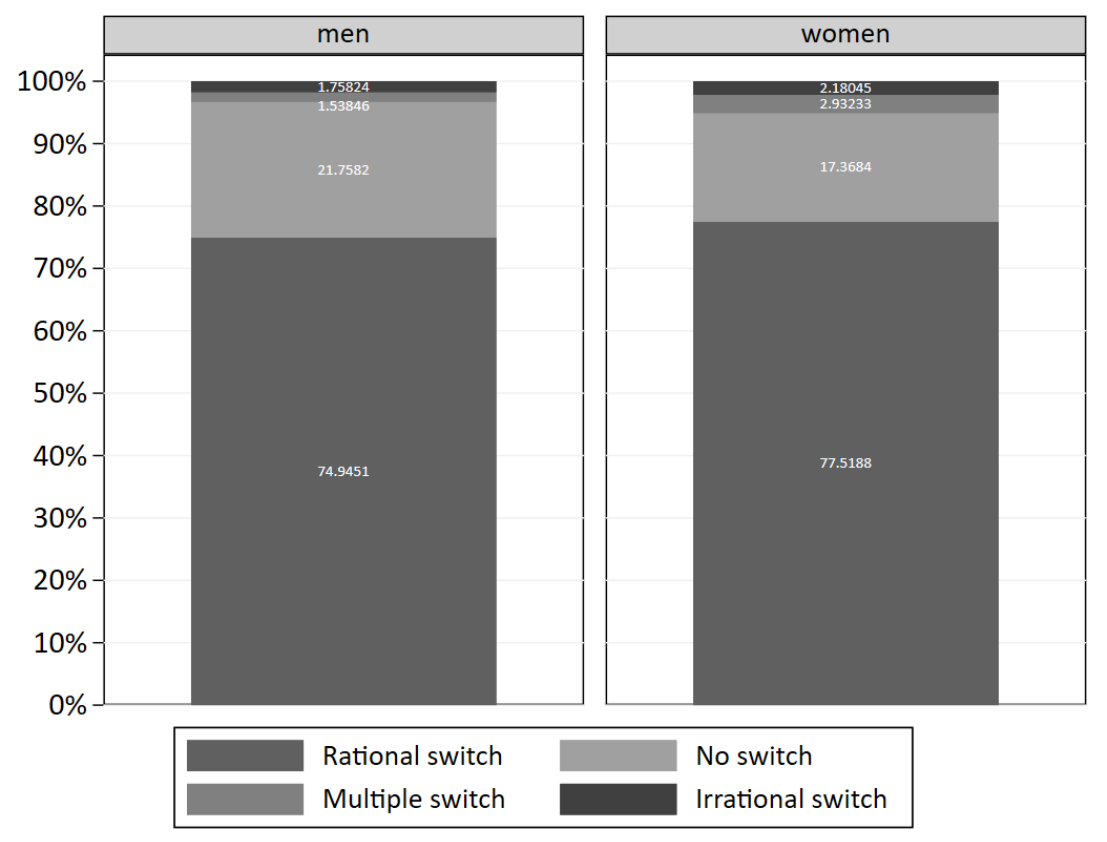

Figure 2.15: Consistency at set level by gender

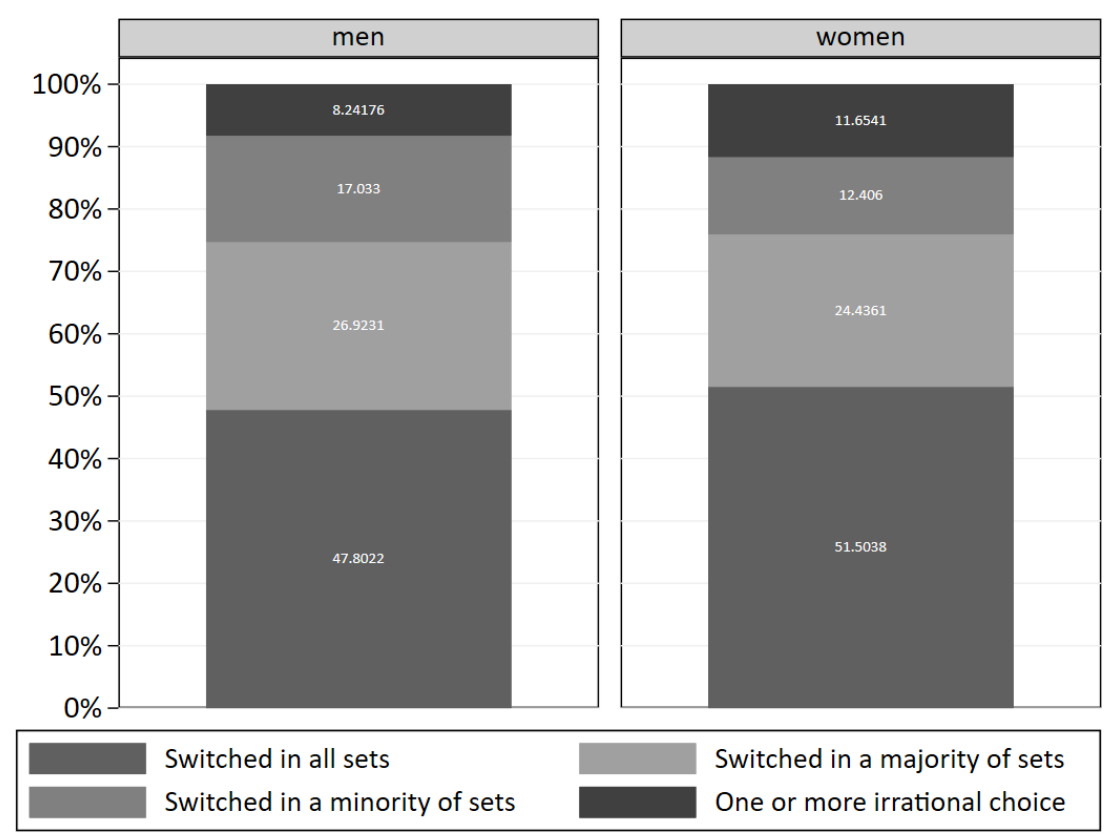

Figure 2.16: Consistency at the individual level by gender 


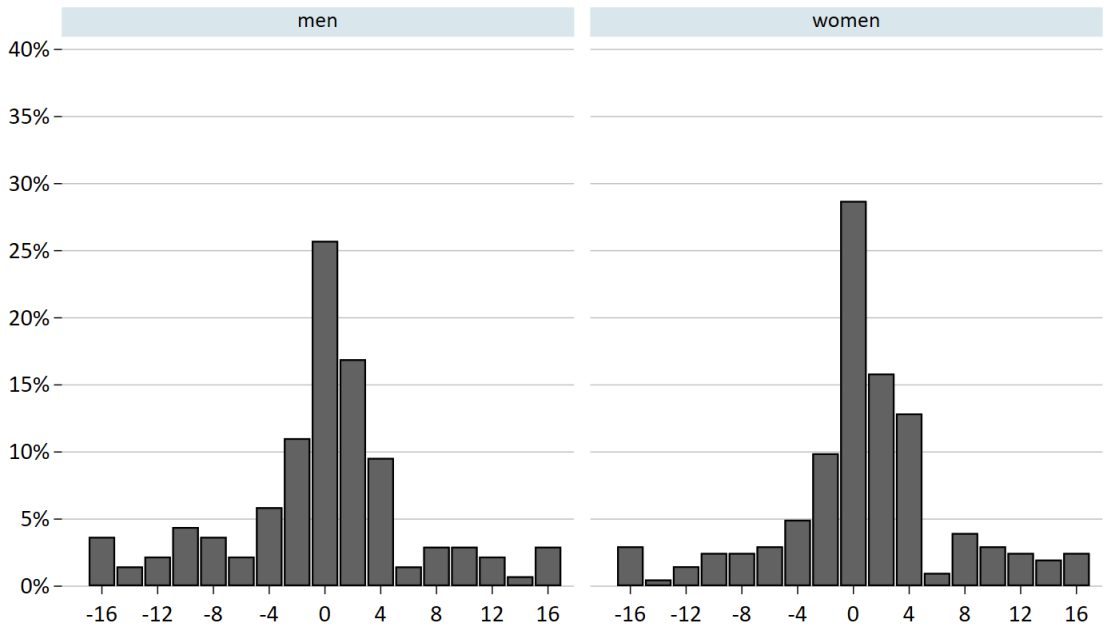

Figure 2.17: Preferences for Competition (in euros) - Distribution of $\omega_{i}$ at the individual level by gender.

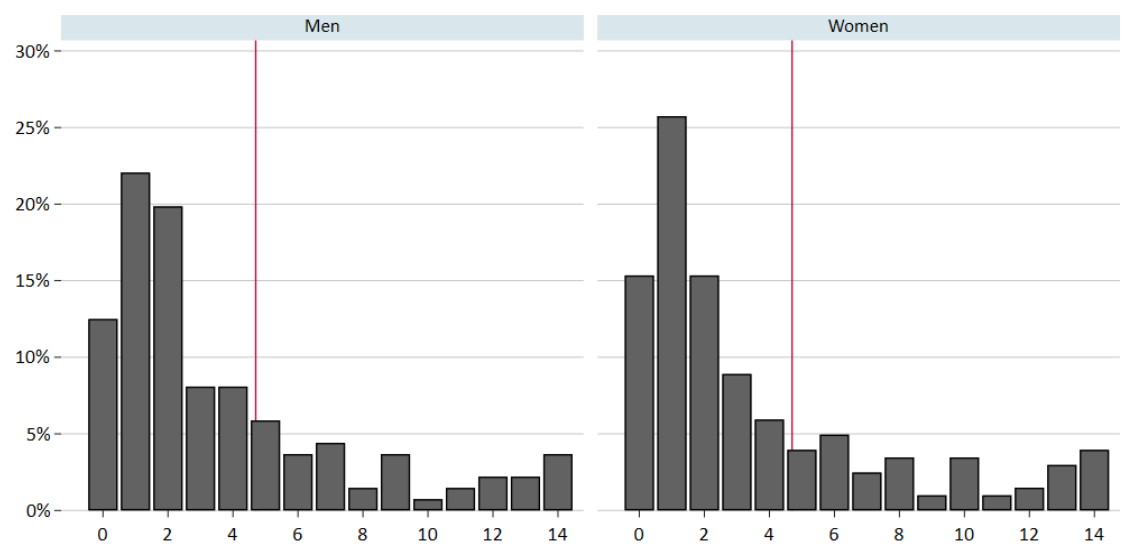

Figure 2.18: Interquartile ranges distribution of Delta at the individual level by gender. 


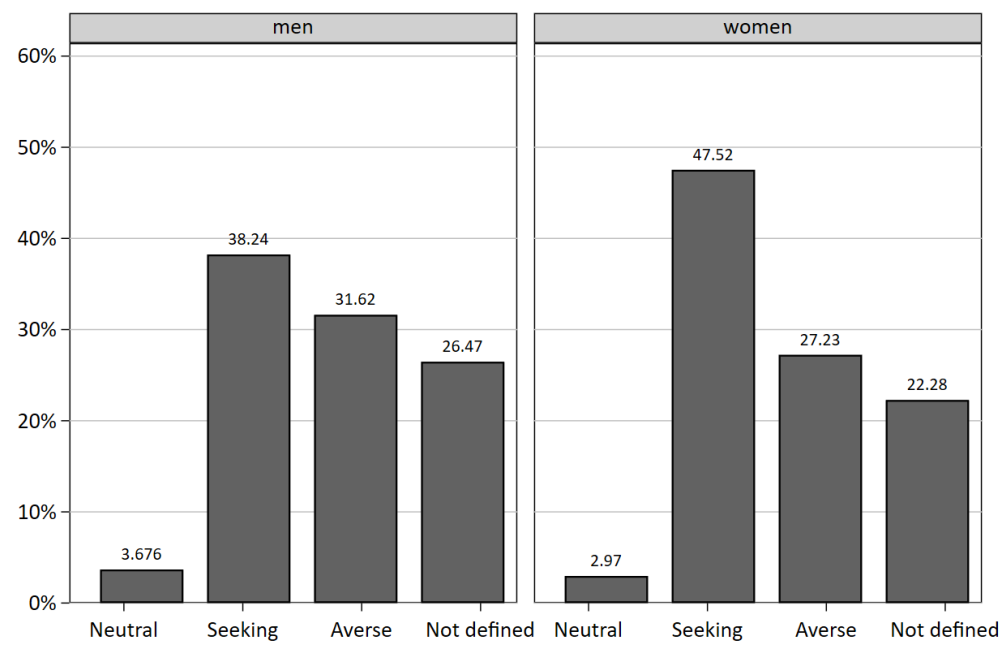

Figure 2.19: Intensity of Preferences for Competition by gender 


\section{A6. Instructions}

\section{General Instructions - both group sizes}

Welcome to the experiment. ${ }^{39}$ In the experiment today, you will be asked to complete five tasks. Before each task, you will receive detailed instructions and description of how your earnings in that task are determined.

Your total earnings at the end of the experiment are the sum of the following two components:

1. A $€ 10$ show-up fee;

2. Your earnings in one of the five tasks.

Specifically, at the end of the experiment, one of the five tasks you will complete during the experiment will be randomly chosen for payment purposes.

During the experiment, the use of cell phones is prohibited. All your information, decisions, and performance during this experiment are anonymous.

If you have a question, please raise your hand. An experimenter will come and answer your question in private.

Now you will start Task 1, please read the instructions of Task 1 carefully.

[new page]

\section{Task 1}

In Task 1, you will be randomly assigned to a group of three[six] participants. In other words, you will be matched with two[five] other participants in the room.

In Task 1 you will be given four minutes to calculate a series of sums of four two-digit numbers (see Figure 1). You cannot use a calculator, but you are welcome to use the provided scratch paper. You submit an answer by clicking the button "Next". When you submit an answer, the computer will immediately tell you whether the answer is correct or incorrect and a new sum is generated.

Your earnings in Task 1 depend on your number of correct sums. Specifically, you can earn either a high amount or a low amount per correct sum. The high amount will vary between $€ 1.5$ and $€ 6$ per correct sum, and the low amount will vary between $€ 0$ and $€ 1$. You will be given the precise values before you perform the task. Whether you are paid a high amount or a low amount depends on your choices. Before you perform the task, you will choose

\footnotetext{
${ }^{39}$ The text font, the size and the appearance of images have been adapted from the original instruction version.
} 


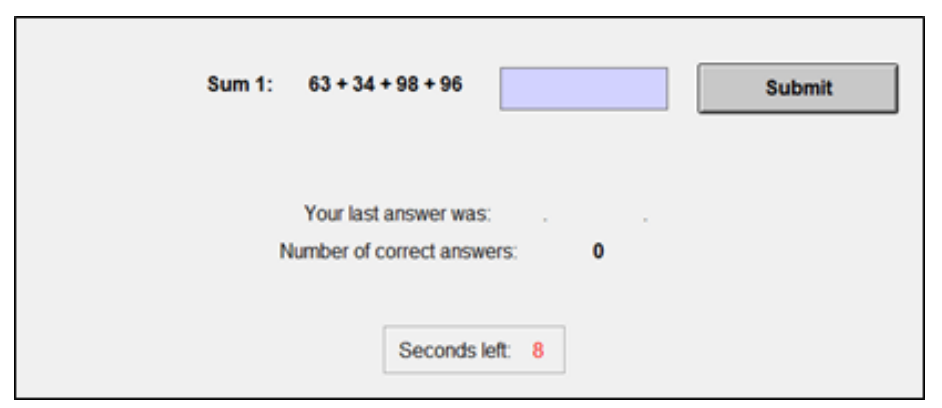

Figure 1

between Individual Pay and Competitive Pay. The two payments schemes are as follows:

Individual Pay: if you choose Individual Pay, whether you receive a high or low amount per correct sum depends on chance. With Individual Pay your earnings do NOT depend on the performance of others in your group.

Competitive Pay: if you choose Competitive Pay, whether you receive a high or low amount per correct sum depends on your performance and the performance of the other two[five] members of your group.

Specifically, you will be your group's winner if you solve more sums in Task 1 than all others in your group in Task 1. If there are ties, the winner will be randomly determined among the tied group members. If you are your group's winner, you will receive the high amount per correct sum.

If you are NOT your group's winner, then you are one of the two losers in the group. If you are one of the group's losers, you will receive the low amount per correct sum.

PRACTICE ROUND: Before Task 1 starts, you will have two minutes to get familiar with the screen and to practice the calculation of series of sums of four two-digit numbers. Please notice that your answers in this practice round will not be considered for your earnings in this experiment.

Once you are done reading, click on the "NEXT" button on your screen. 


\section{TASK 2 - Group of three}

In this task, you can earn money by answering the following question:

How likely do you think it is that you are the winner of your group in Task $1 ?$

Your answer can go from 0 (meaning you are completely certain that you are not the winner of your group) to 100 (meaning you are completely certain that you are the winner of your group). Your earnings in Task 2 can be either $€ 0$ or $€ 20$. The probability of earning $€ 20$ depends on two things:

1. The actual outcome (whether you are the winner or a loser in your group).

2. The likelihood you selected as the answer to the question above.

The closer the likelihood you choose is to your actual outcome in Task 1, the higher the probability you have of earning $€ 20$. This probability is based on the formulas you see in the footnote. ${ }^{40}$ It is not necessary for you to understand precisely the formulas, but it's important that you know that these formulas have been designed so that your expected earnings are higher the closer your answer is to your actual likelihood of being your group's winner.

To help you to think about your likelihood of being your group's winner, it is useful to think how your performance in Task 1 ranks compared to the performance of all participants. The table provided in the next page displays this information. In the table you can see for each possible rank (from being on the top $0 \%$ to being on the top 100\%) the likelihood that someone with that rank is the winner of a group of three.

The numbers on the table are calculated based on you being randomly assigned to a group of three people. For example, imagine that your performance in Task 1 puts you in the Top 10\%. This means that you performed better than $90 \%$ of all participants in the study and you performed worse than around $10 \%$ of all participants in the study. Then for you to be the winner it must be the case that ALL TWO of the other members of your group have a worse rank than you. In other words,

- You have been randomly matched ONLY with participants who ALL come from the $90 \%$ of participants who performed worse than you, and

- You have NOT been randomly matched with ANY of the $10 \%$ of participants who performed better than you. The table shows that, for someone in the Top 10\%, the likelihood that this happens is $81.00 \%$.

\footnotetext{
${ }^{40}$ Probability of earning $€ 20$ if you are the winner $=1-(1-\text { Your selectedlikelihood } / 100)^{2}$ Probability of earning $€ 20$ if you are one of the losers $=1-(\text { Your selectedlikelihood } / 100)^{2}$
} 


\begin{tabular}{|c|c|}
\hline $\begin{array}{l}\text { Your performance } \\
\text { is in the Top .- }\end{array}$ & $\begin{array}{l}\text { The the thood that you are } \\
\text { your group's winner ks- }\end{array}$ \\
\hline $0 \%$ & $10000 \%$ \\
\hline $1 \%$ & $98.01 \%$ \\
\hline $2 \%$ & $96.04 \%$ \\
\hline $3 \%$ & $94.09 \%$ \\
\hline $4 \%$ & $92.16 \%$ \\
\hline $5 \%$ & $50.25 \%$ \\
\hline $6 \%$ & $8835 \%$ \\
\hline $7 \%$ & $86.49 \%$ \\
\hline $8 \%$ & $84.64 \%$ \\
\hline $9 \%$ & $8281 \%$ \\
\hline $105 \%$ & $81.00 \%$ \\
\hline $11 \%$ & $79.21 \%$ \\
\hline $127 \%$ & $77.44 \%$ \\
\hline $13 \%$ & $75.69 \%$ \\
\hline 1636 & $7395 \%$ \\
\hline $15 \%$ & $7225 \%$ \\
\hline $16 \% 6$ & $70.56 \%$ \\
\hline $17 \%$ & $68.89 \%$ \\
\hline $18 \%$ & $67.24 \%$ \\
\hline $19 \% 6$ & $65.61 \%$ \\
\hline $20 \%$ & $64.00 \%$ \\
\hline $21 \%$ & $62.41 \%$ \\
\hline $227 \%$ & $60.84 \%$ \\
\hline 2336 & $59.29 \%$ \\
\hline $26 \%$ & $57.76 \%$ \\
\hline $25 \%$ & $56.25 \%$ \\
\hline 2676 & $54.76 \%$ \\
\hline $27 \%$ & $53.29 \%$ \\
\hline $28 \%$ & $51.84 \%$ \\
\hline $29 \%$ & $50.41 \%$ \\
\hline 3056 & $49.00 \%$ \\
\hline $31 \%$ & $47.61 \%$ \\
\hline $327 \%$ & $46.24 \%$ \\
\hline $33 \%$ & $44.89 \%$ \\
\hline 3676 & $43.56 \%$ \\
\hline $35 \% 6$ & $42.25 \%$ \\
\hline $36 \%$ & $40.96 \%$ \\
\hline $37 \%$ & $39.69 \%$ \\
\hline 3836 & $38.44 \%$ \\
\hline 3996 & $37.21 \%$ \\
\hline $40 \%$ & $36.00 \%$ \\
\hline $41 \%$ & $34.81 \%$ \\
\hline $427 \%$ & $33.64 \%$ \\
\hline 4336 & $32.49 \%$ \\
\hline $46 \%$ & $3136 \%$ \\
\hline $45 \%$ & $30.25 \%$ \\
\hline 4676 & $29.16 \%$ \\
\hline $47 \% 6$ & $28.09 \%$ \\
\hline 4836 & $27.04 \%$ \\
\hline \multirow[t]{2}{*}{$49 \%$} & $26.01 \%$ \\
\hline & continues $\rightarrow$ \\
\hline
\end{tabular}

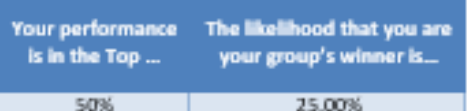

\begin{tabular}{|c|c|}
\hline $50 \% 6$ & $2500 \%$ \\
\hline 5130
\end{tabular}

\begin{tabular}{|c|c|}
\hline $51 \%$ & $24.01 \%$ \\
\hline $527 \%$ & $23.04 \%$ \\
\hline $53 \%$ & $2209 \%$ \\
\hline $56 \%$ & $21.16 \%$ \\
\hline $55 \%$
\end{tabular}

\begin{tabular}{|c|c|}
\hline $56 \%$ & $21.16 \%$ \\
\hline $55 \%$ & $20.25 \%$ \\
\hline $56 \% \%$ & $19.35 \%$ \\
\hline $57 \%$ & $18.49 \%$ \\
\hline $58 \%$ & $17.64 \%$ \\
\hline $59 \%$
\end{tabular}

\begin{tabular}{|c|c|}
\hline $58 \% \%$ & $17.64 \%$ \\
\hline $59 \%$ & $16.81 \%$ \\
\hline $600 \%$ & $16.00 \%$ \\
\hline $61 \%$ & $15.21 \%$ \\
\hline
\end{tabular}

\begin{tabular}{|l|l|}
\hline $61 \%$ & $15.21 \%$ \\
\hline $62 \%$ & $14.44 \%$ \\
\hline $63 \%$ & $13.69 \%$ \\
\hline $64 \%$ & $12.96 \%$ \\
\hline $65 \%$ & $1225 \%$ \\
\hline
\end{tabular}

\begin{tabular}{|c|c|}
\hline $65 \%$ & $1296 \%$ \\
\hline $65 \%$ & $12.25 \%$ \\
\hline $67 \%$ & $11.56 \%$ \\
\hline $68 \%$ & $10.89 \%$ \\
\hline $69 \%$ & $10.24 \%$ \\
\hline
\end{tabular}

\begin{tabular}{|c|c|}
\hline & \\
\hline $69 \%$ & $9.61 \%$ \\
\hline $70 \% 6$ & $9.00 \%$ \\
\hline $71 \%$ & $8.41 \%$ \\
\hline 7236 & $7.84 \%$ \\
\hline $73 \%$ & $7.29 \%$ \\
\hline $74 \%$ & $6.75 \%$ \\
\hline $75 \%$ & $6.25 \%$ \\
\hline $76 \%$ & $5.76 \% 6$ \\
\hline $77 \%$ & $5.29 \%$ \\
\hline $78 \%$ & $4.84 \%$ \\
\hline $79 \%$ & $4.41 \%$ \\
\hline $80 \%$ & $4.00 \%$ \\
\hline $81 \%$ & $3.61 \%$ \\
\hline $82 \%$ & $3.24 \%$ \\
\hline $83 \%$ & $2.89 \%$ \\
\hline $86 \%$ & $2.56 \%$ \\
\hline $85 \%$ & $2.25 \%$ \\
\hline $86 \%$ & $1.96 \%$ \\
\hline $87 \%$ & $1.69 \%$ \\
\hline $85 \% 6$ & $1.44 \%$ \\
\hline $89 \%$ & $1.21 \%$ \\
\hline $90 \%$ & $1.00 \%$ \\
\hline $91 \%$ & $0.81 \%$ \\
\hline $92 \%$ & $0.64 \%$ \\
\hline $93 x^{\circ}$ & $0.49 \%$ \\
\hline $96 \%$ & $0.35 \%$ \\
\hline $95 \%$ & $0.25 \%$ \\
\hline $96 \%$ & $0.16 \%$ \\
\hline $97 \%$ & $0.09 \%$ \\
\hline $98 \%$ & $0.04 \%$ \\
\hline $99 \%$ & $0.01 \%$ \\
\hline $100 \%$ & $0.00 \%$ \\
\hline
\end{tabular}

Figure 2.20: Ranking table

You will indicate your likelihood of being your group's winner in a screen like the one below. As you can see, there are two sliders in the top part of the screen. You can select your answer by moving the cursors in these two different sliders:

- In the black slider, you can select your likelihood of being the winner of your group. Your answer can go from 0\% (meaning you are completely certain that you are not the winner of your group) to 100\% (meaning you are completely certain that you are the winner of your group).

- In the green slider, you can select how your performance in task 1 ranks compared to the performance of all participants. 
Your answer can go from Top 100\% (you performed worse than ALL other participants of the study) to Top 0\% (you performed better than ALL other participants in the study).

Please notice that the information displayed in both sliders is always consistent with each other. In other words, when you select a likelihood on the black slider, the cursor on the green slider will automatically mark the rank associated with your selected likelihood. Similarly, when you select a rank on the green slider, the cursor on the black slider will automatically mark the likelihood associated with your selected rank. The values of the sliders are based on the numbers you can see in the table of the previous page.

The cursors will appear on the sliders only after you have clicked on one of the sliders for the first time.

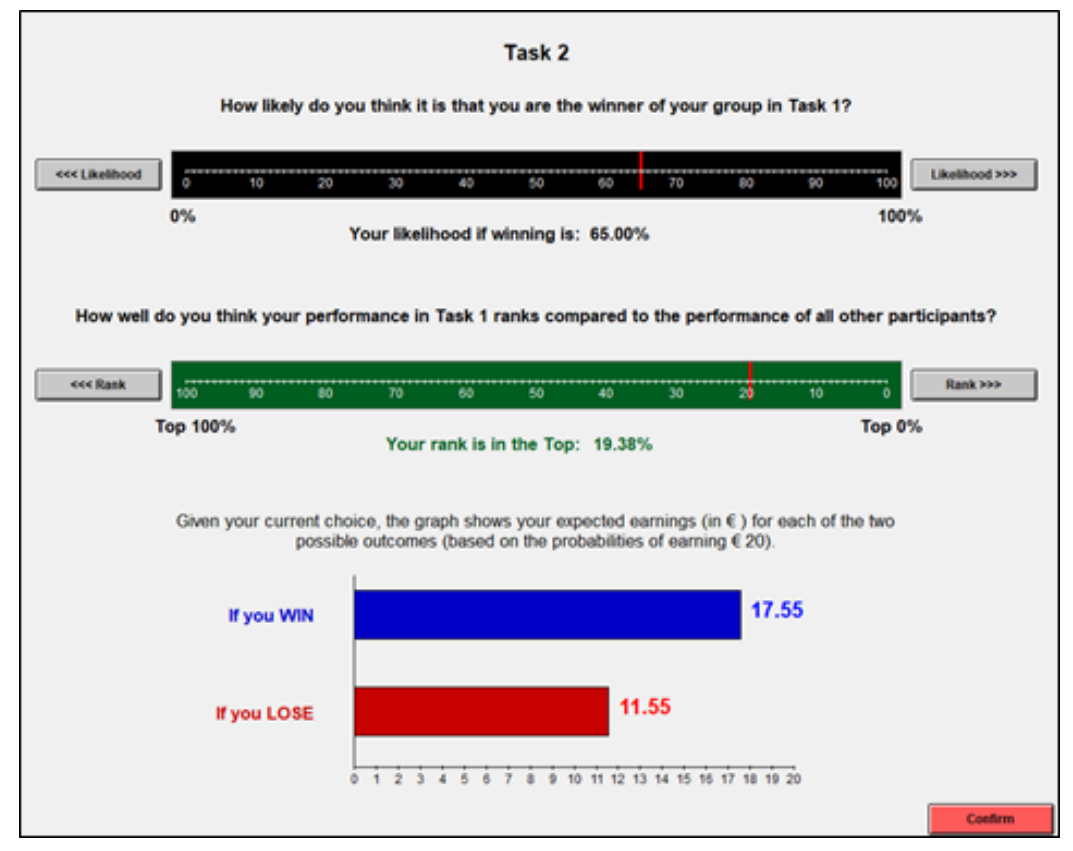

Figure 2.21: Task 2 - Screen example 
To help you to understand the consequences of your choice, below the sliders, you will also see the expected earnings associated to your choice in the two possible outcomes: in case you are the winner, and in case you are one of the losers of your group. You will obtain the highest expected earnings if your answer equals the actual likelihood of you being the winner.

Please remember that your earnings in Task 2 are either $€ 0$ or $€ 20$, therefore, your expected earnings are equal to $€ 20$ multiplied by the probability of earning the $€ 20$ (which is calculated with the formulas in footnote 1 ).

We provide an example below to illustrate how your earnings depend on your answers (note that the numbers used in this example are not indicative of what constitutes a good or bad answer in this task).

\section{Example}

Imagine that among the students taking part in this study, your performance in Task 1 puts you in the Top $\mathbf{3 0 \%}$. In other words, $70 \%$ of the study participants performed worse than you did and 30\% performed better than you did. Recall that, for you to be the group's winner, it must be the case that ALL TWO of the other members of your group come from the $70 \%$ of participants who performed worse than you did. In this example, the probability that this occurs is $49.00 \%$ (see the table).

Suppose that your answer is $49.00 \%$ in the black slider and Top 30\% in the green slider, as shown in the screen below.

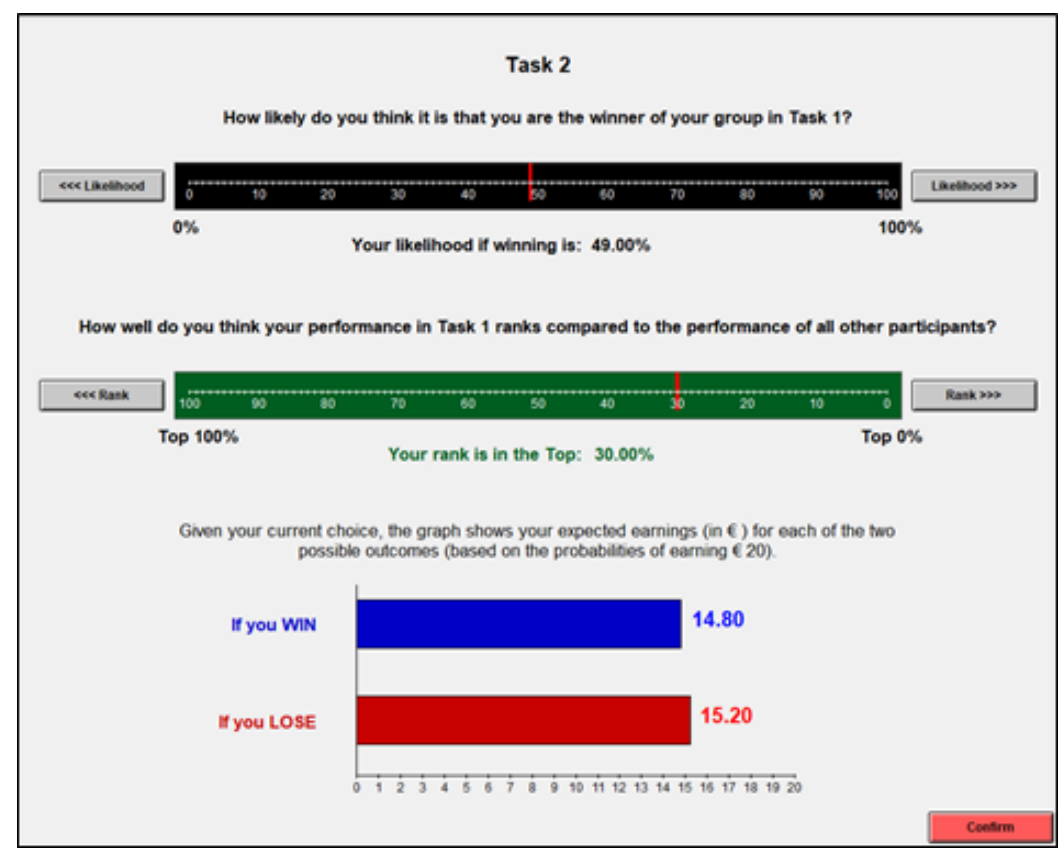


Then, as you can see with the bar graph in the screenshot:

- If you turn out to be the winner of your group, you can expect to earn in Task $2 € 14.80$ on average ( $=€ 20 \times$ probability of earning $€ 20$ if you are the winner).

- If you turn out to be one of the losers of your group, you can expect to earn in Task $2 € 15.20$ on average $(=€ 20 \times$ probability of earning $€ 20$ if you are one of the losers).

Since the actual likelihood that you are the winner of your group is $49.00 \%$, this means that $49.00 \%$ of the time you are the group's winner and $51.00 \%$ of the time you are one of the losers. Overall, this means that you can expect to earn in Task $2 € \mathbf{1 5 . 0 0}$ on average $(€ 15.00=0.49 \times € 14.80+0.51 \times$ $€ 15.20)$.

Now let's see what happens if you answer differently.

Continue to suppose that your performance places you in the Top $\mathbf{3 0 \%}$. However, imagine that this time your answer is $77.44 \%$ in the black slider and Top $12 \%$ in the green slider, as shown in the screen below.

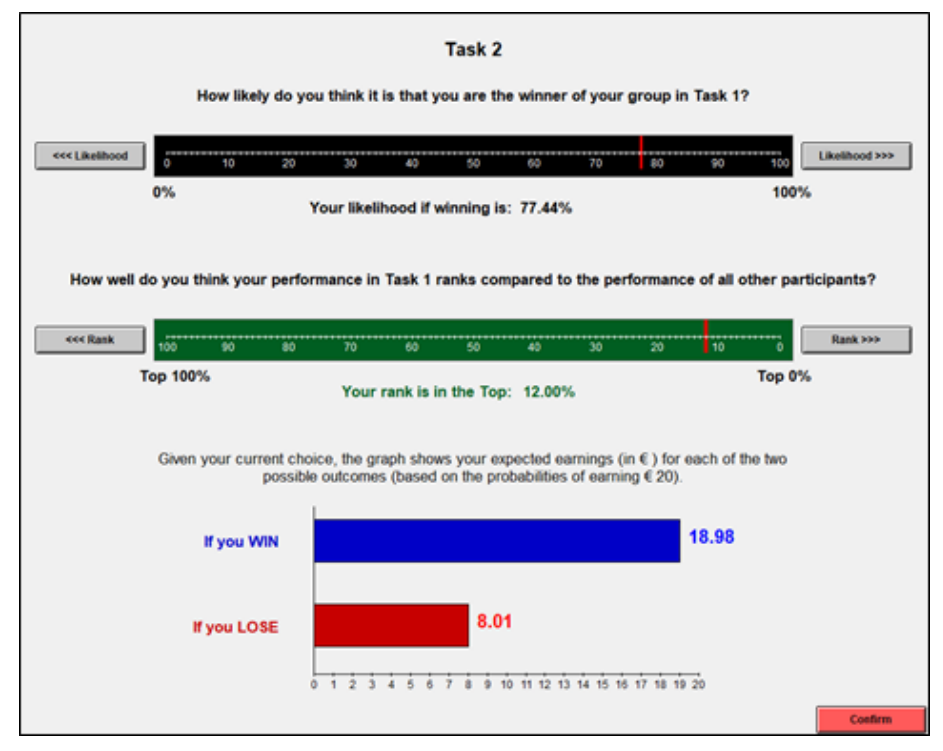

Then, as you can see with the bar graph in the screenshot:

- If you turn out to be the winner of your group, you can expect to earn in Task $2 € 18.98$ on average ( $€ 20 \times$ probability of earning $€ 20$ if you are the winner).

- If you turn out to be one of the losers of your group, you can expect to earn in Task $2 € 8.01$ on average ( $€ 20 \times$ probability of earning $€ 20$ if you are one of the losers). 
Since the actual likelihood that you are the winner of your group is still $49.00 \%$ (remember that you actually are in the Top 30\%), this means you can expect to earn in Task $2 € 13.39$ on average $(€ 13.39=0.49 \times € 18.98+$ $0.51 \times € 8.01)$.

Note that $€ \mathbf{1 3 . 3 9}$ is lower than $€ \mathbf{1 5 . 0 0}$, which are the expected earnings from reporting $49.00 \%$ in the black slider and Top 30\% in the green slider.

In conclusion and to reiterate, you will obtain the highest expected earnings in Task 2 if your answer equals your actual likelihood of being the group's winner in Task 1.

Once you are done reading, click on the "Next" button on your screen. 


\section{TASK 2 - Group of six}

In this task, you can earn money by answering the following question:

How likely do you think it is that you are the winner of your group in Task $1 ?$

Your answer can go from 0 (meaning you are completely certain that you are not the winner of your group) to 100 (meaning you are completely certain that you are the winner of your group). Your earnings in Task 2 can be either $€ 0$ or $€ 20$. The probability of earning $€ 20$ depends on two things:

1. The actual outcome (whether you are the winner or a loser in your group).

2. The likelihood you selected as the answer to the question above.

The closer the likelihood you choose is to your actual outcome in Task 1, the higher the probability you have of earning $€ 20$. This probability is based on the formulas you see in the footnote. ${ }^{41}$ It is not necessary for you to understand precisely the formulas, but it's important that you know that these formulas have been designed so that your expected earnings are higher the closer your answer is to your actual likelihood of being your group's winner.

To help you to think about your likelihood of being your group's winner, it is useful to think how your performance in Task 1 ranks compared to the performance of all participants. The table provided in the next page displays this information. In the table you can see for each possible rank (from being on the top $0 \%$ to being on the top 100\%) the likelihood that someone with that rank is the winner of a group of three.

The numbers on the table are calculated based on you being randomly assigned to a group of six people. For example, imagine that your performance in Task 1 puts you in the Top 10\%. This means that you performed better than $90 \%$ of all participants in the study and you performed worse than around $10 \%$ of all participants in the study. Then for you to be the winner it must be the case that ALL FIVE of the other members of your group have a worse rank than you. In other words,

- You have been randomly matched ONLY with participants who ALL come from the $90 \%$ of participants who performed worse than you, and

- You have NOT been randomly matched with ANY of the $10 \%$ of participants who performed better than you. The table shows that, for someone in the Top $10 \%$, the likelihood that this happens is $81.00 \%$.

\footnotetext{
${ }^{41}$ Probability of earning $€ 20$ if you are the winner $=1-(1-\text { Your selectedlikelihood } / 100)^{2}$ Probability of earning $€ 20$ if you are one of the losers $=1-(\text { Your selectedlikelihood } / 100)^{2}$
} 


\begin{tabular}{|c|c|}
\hline $\begin{array}{l}\text { Your performance } \\
\text { is in the Top ... }\end{array}$ & $\begin{array}{l}\text { The thel hood that you are } \\
\text { your group's winner k- }\end{array}$ \\
\hline $0 \%$ & $100000 \%$ \\
\hline $1 \%$ & 95.10\% \\
\hline $2 \%$ & $5039 \%$ \\
\hline $3 \%$ & $85.87 \%$ \\
\hline $4 \%$ & $8154 \%$ \\
\hline $5 \%$ & $7738 \%$ \\
\hline $6 \%$ & $73.39 \%$ \\
\hline $7 \%$ & $69.57 \%$ \\
\hline $8 \%$ & $65.91 \%$ \\
\hline $9 \%$ & $62.40 \%$ \\
\hline $10 \% 6$ & $59.05 \%$ \\
\hline $11 \%$ & $55.84 \%$ \\
\hline $127 \%$ & $52.77 \%$ \\
\hline $13 \%$ & $49.84 \%$ \\
\hline $16 \%$ & $4704 \%$ \\
\hline $15 \%$ & $44.37 \% 6$ \\
\hline $16 \%$ & $4182 \%$ \\
\hline $17 \% 6$ & $3939 \%$ \\
\hline $18 \% 6$ & $37.07 \%$ \\
\hline $19 \%$ & $34.87 \%$ \\
\hline $20 \% 6$ & $32.77 \%$ \\
\hline $21 \%$ & $30.77 \%$ \\
\hline $227 \%$ & $28.87 \%$ \\
\hline $23 \%$ & $27.07 \%$ \\
\hline $24 \% 6$ & $25.35 \%$ \\
\hline $25 \%$ & $23.73 \%$ \\
\hline $26 \%$ & $22.19 \%$ \\
\hline $27 \%$ & $20.73 \%$ \\
\hline $28 \%$ & $1935 \%$ \\
\hline $29 \%$ & $18.04 \%$ \\
\hline $30 \%$ & $1681 \%$ \\
\hline $31 \%$ & $15.64 \%$ \\
\hline $32 \%$ & $14.54 \%$ \\
\hline $33 \%$ & $1350 \%$ \\
\hline $36 \%$ & $1252 \%$ \\
\hline $35 \%$ & $11.60 \%$ \\
\hline $36 \%$ & $10.74 \%$ \\
\hline $37 \%$ & $9.92 \%$ \\
\hline $38 \%$ & $9.16 \%$ \\
\hline $39 \%$ & $8.45 \%$ \\
\hline $40 \%$ & $7.78 \%$ \\
\hline $41 \%$ & $7.15 \%$ \\
\hline $427 \%$ & $6.56 \%$ \\
\hline $43 \%$ & $6.02 \%$ \\
\hline $46 \%$ & $5.51 \%$ \\
\hline $45 \%$ & $5.03 \%$ \\
\hline $46 \%$ & $4.59 \%$ \\
\hline $47 \%$ & $4.18 \%$ \\
\hline $48 \%$ & $3.80 \%$ \\
\hline \multirow[t]{2}{*}{$49 \%$} & $3.45 \%$ \\
\hline & continues $\rightarrow$ \\
\hline
\end{tabular}

\begin{tabular}{|c|c|}
\hline $\begin{array}{l}\text { Your performance } \\
\text { Is in the Top ... }\end{array}$ & $\begin{array}{l}\text { The thelhood that you are } \\
\text { your group's winner k- }\end{array}$ \\
\hline $500 \%$ & $3.13 \%$ \\
\hline $51 \%$ & $2.82 \%$ \\
\hline $52 \%$ & $2.55 \%$ \\
\hline $53 \%$ & $2.29 \%$ \\
\hline $56 \%$ & $2.05 \%$ \\
\hline $55 \%$ & $1.85 \%$ \\
\hline $56 \%$ & $1.65 \%$ \\
\hline $57 \% 6$ & $1.47 \%$ \\
\hline $58 \%$ & $1.31 \%$ \\
\hline $59 \%$ & $1.16 \%$ \\
\hline $60 \%$ & $1.02 \%$ \\
\hline $61 \%$ & $0.90 \%$ \\
\hline $62 \%$ & $0.79 \%$ \\
\hline $63 \%$ & $0.69 \%$ \\
\hline $64 \%$ & $0.60 \%$ \\
\hline $65 \%$ & $0.53 \%$ \\
\hline $66 \%$ & $0.45 \%$ \\
\hline $67 \%$ & $0.39 \%$ \\
\hline $68 \% 6$ & $0.34 \%$ \\
\hline $69 \%$ & $0.29 \%$ \\
\hline $70 \% 6$ & $0.24 \%$ \\
\hline $71 \%$ & $0.21 \%$ \\
\hline $72 \%$ & $0.17 \%$ \\
\hline $73 \%$ & $0.14 \%$ \\
\hline $74 \%$ & $0.12 \%$ \\
\hline $75 \%$ & $0.10 \%$ \\
\hline $76 \%$ & $0.08 \%$ \\
\hline $77 \%$ & $0.05 \%$ \\
\hline $78 \% 6$ & $0.05 \%$ \\
\hline $79 \%$ & $0.04 \%$ \\
\hline $80 \%$ & $0.03 \%$ \\
\hline $81 \%$ & $0.02 \%$ \\
\hline $82 \% 6$ & $0.02 \%$ \\
\hline $83 \% 6$ & $0.01 \%$ \\
\hline $86 \%$ & $0.01 \%$ \\
\hline $85 \%$ & $0.01 \%$ \\
\hline $86 \%$ & $0.01 \%$ \\
\hline $87 \% 6$ & $0.00 \%$ \\
\hline $88 \% 6$ & $0.00 \%$ \\
\hline $89 \%$ & $0.00 \%$ \\
\hline $90 \% 6$ & $0.00 \%$ \\
\hline $91 \%$ & $0.00 \%$ \\
\hline 9276 & $0.00 \%$ \\
\hline $93 \%$ & $0.00 \%$ \\
\hline $96 \%$ & $0.00 \%$ \\
\hline $95 \%$ & $0.00 \%$ \\
\hline $96 \%$ & $0.00 \%$ \\
\hline $97 \%$ & $0.00 \%$ \\
\hline $98 \%$ & $0.00 \%$ \\
\hline $99 \% 6$ & $0.00 \%$ \\
\hline $100 \%$ & $0.00 \%$ \\
\hline
\end{tabular}

You will indicate your likelihood of being your group's winner in a screen like the one below. As you can see, there are two sliders in the top part of the screen. You can select your answer by moving the cursors in these two different sliders:

- In the black slider, you can select your likelihood of being the winner of your group. Your answer can go from 0\% (meaning you are completely certain that you are not the winner of your group) to 100\% (meaning you are completely certain that you are the winner of your group).

- In the green slider, you can select how your performance in task 1 ranks compared to the performance of all participants. 
Your answer can go from Top 100\% (you performed worse than ALL other participants of the study) to Top 0\% (you performed better than ALL other participants in the study).

Please notice that the information displayed in both sliders is always consistent with each other. In other words, when you select a likelihood on the black slider, the cursor on the green slider will automatically mark the rank associated with your selected likelihood. Similarly, when you select a rank on the green slider, the cursor on the black slider will automatically mark the likelihood associated with your selected rank. The values of the sliders are based on the numbers you can see in the table of the previous page.

The cursors will appear on the sliders only after you have clicked on one of the sliders for the first time.

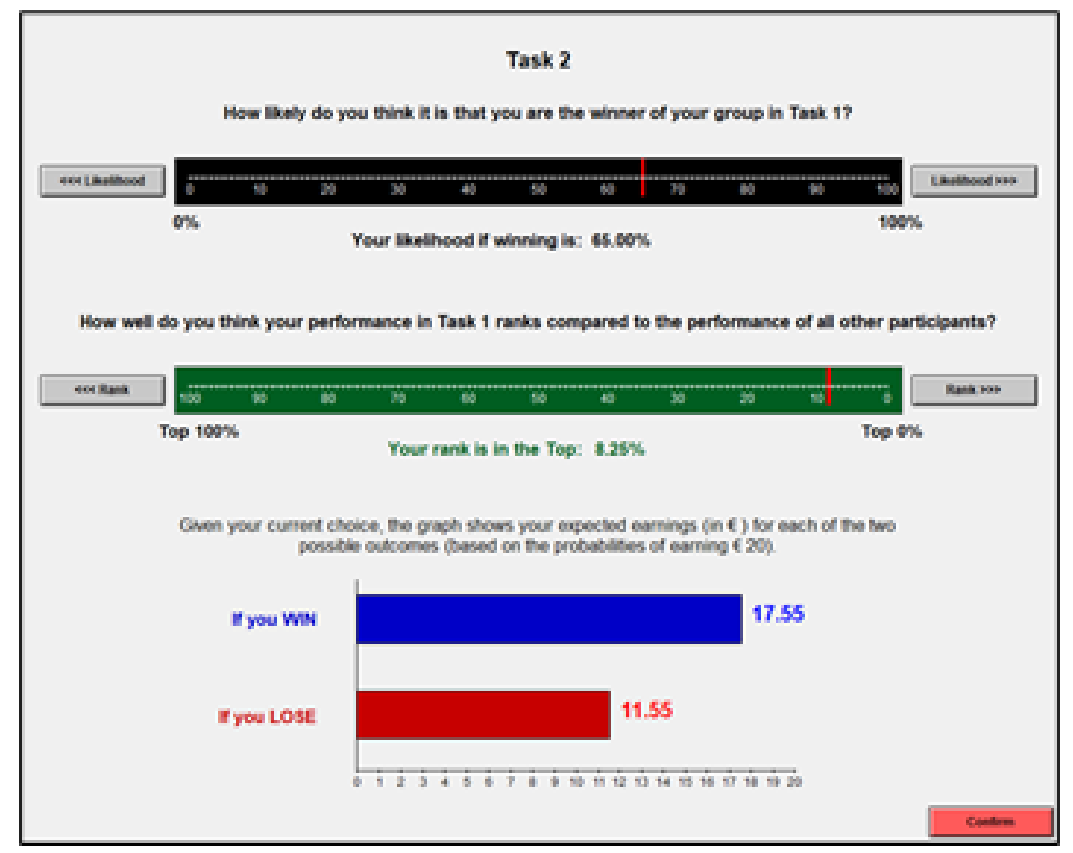


To help you to understand the consequences of your choice, below the sliders, you will also see the expected earnings associated to your choice in the two possible outcomes: in case you are the winner, and in case you are one of the losers of your group. You will obtain the highest expected earnings if your answer equals the actual likelihood of you being the winner.

Please remember that your earnings in Task 2 are either $€ 0$ or $€ 20$, therefore, your expected earnings are equal to $€ 20$ multiplied by the probability of earning the $€ 20$ (which is calculated with the formulas in footnote 1 ).

We provide an example below to illustrate how your earnings depend on your answers (note that the numbers used in this example are not indicative of what constitutes a good or bad answer in this task).

\section{Example}

Imagine that among the students taking part in this study, your performance in Task 1 puts you in the Top $\mathbf{3 0 \%}$. In other words, $70 \%$ of the study participants performed worse than you did and 30\% performed better than you did. Recall that, for you to be the group's winner, it must be the case that ALL FIVE of the other members of your group come from the $70 \%$ of participants who performed worse than you did. In this example, the probability that this occurs is $16.81 \%$ (see the table).

Suppose that your answer is $16.81 \mathrm{~S} \%$ in the black slider and Top $30 \%$ in the green slider, as shown in the screen below.

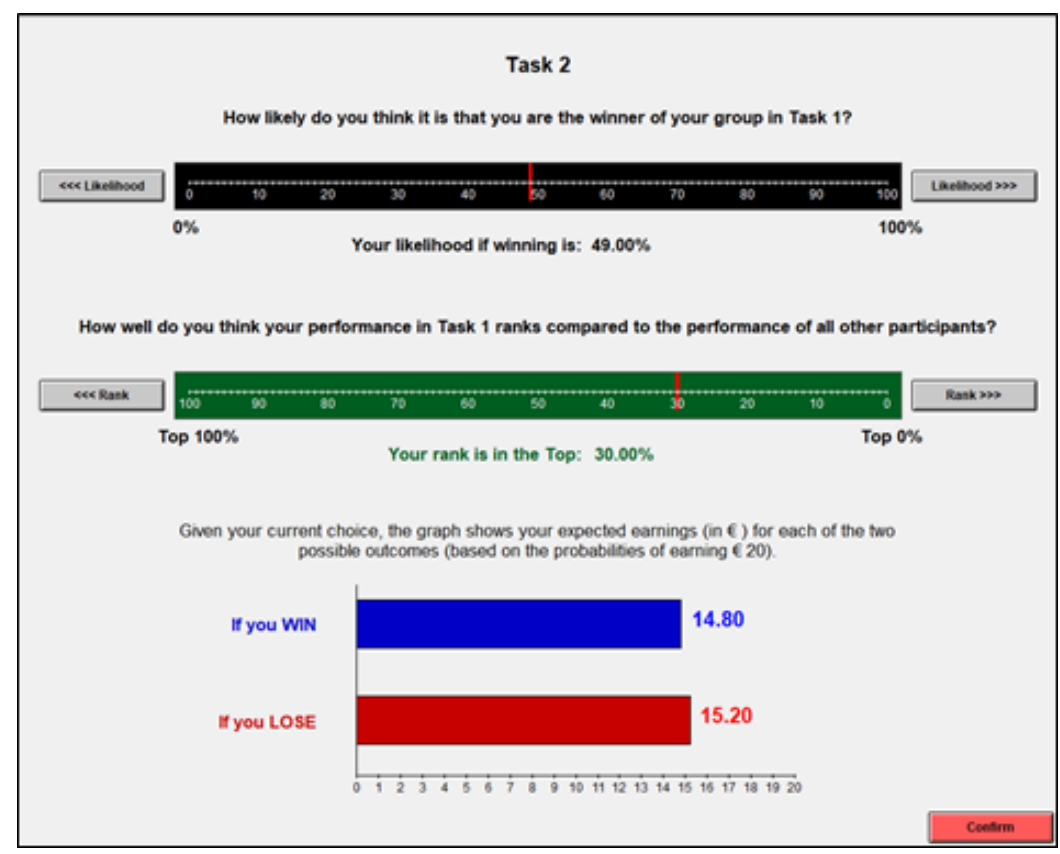


Then, as you can see with the bar graph in the screenshot:

- If you turn out to be the winner of your group, you can expect to earn in Task $2 € 6.16$ on average $(=€ 20 \times$ probability of earning $€ 20$ if you are the winner).

- If you turn out to be one of the losers of your group, you can expect to earn in Task $2 € 19.43$ on average $(=€ 20 \times$ probability of earning $€ 20$ if you are one of the losers).

Since the actual likelihood that you are the winner of your group is $16.81 \%$, this means that $16.81 \%$ of the time you are the group's winner and $83.19 \%$ of the time you are one of the losers. Overall, this means that you can expect to earn in Task $2 € \mathbf{1 7 . 2 0}$ on average $(€ 15.00=0.1681 \times € 6.16+0.8319 \times$ $€ 19.43)$.

Now let's see what happens if you answer differently.

Continue to suppose that your performance places you in the Top $\mathbf{3 0 \%}$. However, imagine that this time your answer is $52.77 \%$ in the black slider and Top $12 \%$ in the green slider, as shown in the screen below.

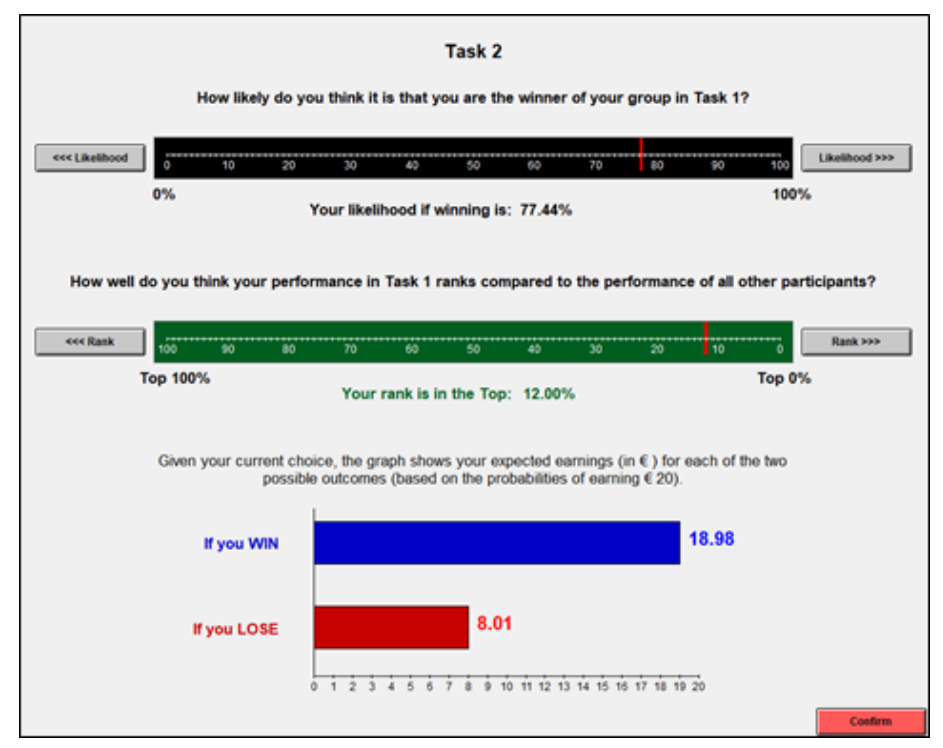

Then, as you can see with the bar graph in the screenshot:

- If you turn out to be the winner of your group, you can expect to earn in Task $2 € 15.54$ on average ( $€ 20 \times$ probability of earning $€ 20$ if you are the winner).

- If you turn out to be one of the losers of your group, you can expect to earn in Task $2 € € 14.43$ on average ( $€ 20 \times$ probability of earning $€ 20$ if you are one of the losers). 
Since the actual likelihood that you are the winner of your group is still $16.81 \%$ (remember that you actually are in the Top 30\%), this means you can expect to earn in Task $2 € \mathbf{1 5 . 0 2}$ on average $(€ 15.02=0.1681 \times € 15.54+$ $0.8319 \times € 14.43)$.

Note that $€ \mathbf{1 5 . 0 2}$ is lower than $€ \mathbf{1 7 . 2 0}$, which are the expected earnings from reporting $16.81 \%$ in the black slider and Top 30\% in the green slider.

In conclusion and to reiterate, you will obtain the highest expected earnings in Task 2 if your answer equals your actual likelihood of being the group's winner in Task 1.

Once you are done reading, click on the "Next" button on your screen. 


\section{YOUR PAYMENT CHOICE IN TASK 1 - both group sizes}

Next you are going to perform Task 1, but before performing the task, you must choose how you want to be paid for each correct sum in Task 1. Recall that you can choose between Individual Pay and Competitive Pay.

You will be asked to make choices in 5 different decision sets. All these decision sets are completely independent of each other. An example of one decision set is displayed in the screenshot below.

\begin{tabular}{|c|c|c|}
\hline & Competitive Pay & Individual Pay \\
\hline 1. & $\epsilon 4.00$ if you win and $\epsilon 1.00$ if you lose & co 64.00 with $17 \%$ chance and 61.00 with $83 \%$ chance \\
\hline 2. & $\epsilon 4.00$ if you win and $\epsilon 1.00$ if you lose & cr $€ 4.00$ with $20 \%$ chance and $€ 1.00$ with $80 \%$ chance \\
\hline 3. & $\epsilon 4.00$ if you win and $\epsilon 1.00$ if you lose & $c r \in 4.00$ with $23 \%$ chance and $\epsilon 1.00$ with $77 \%$ chance \\
\hline 4. & $\epsilon 4.00$ if you win and $\epsilon 1.00$ if you lose & C $\subset \in 4.00$ with $26 \%$ chance and $\ell 1.00$ with $74 \%$ chance \\
\hline 5. & $\epsilon 4.00$ if you win and $\epsilon 1.00$ if you lose & $c r \ell 4.00$ with $29 \%$ chance and $\ell 1.00$ with $71 \%$ chance \\
\hline 6. & $C 4.00$ if you win and $\ell .00$ if you lose & $c r \ell 4.00$ with $32 \%$ chance and $\ell 1.00$ with $68 \%$ chance \\
\hline 7. & $\epsilon 4.00$ if you win and $\ell .00$ if you lose & $C r \in 4.00$ with $35 \%$ chance and $\ell 1.00$ with $65 \%$ chance \\
\hline 8. & $\ell .00$ if you win and $\ell 1.00$ if you lose & cr $\ell 4.00$ with $38 \%$ chance and $\ell 1.00$ with $62 \%$ chance \\
\hline 9. & $\epsilon 4.00$ if you win and $\ell 1.00$ if you lose & C $C \in 4.00$ with $41 \%$ chance and $\ell 1.00$ with $69 \%$ chance \\
\hline 10. & $\epsilon 4.00$ if you win and $\epsilon 1.00$ if you lose & if $\ell 4.00$ with $44 \%$ chance and $\ell 1.00$ with $56 \%$ chance \\
\hline
\end{tabular}

Each decision set consists of a table with a series of choices:

- The left-choices correspond to Competitive Pay. Under Competitive Pay your earnings in Task 1 depend on your performance and the performance of others in your group. Specifically, if are the winner of your group then you earn the high amount per correct sum, otherwise you earn the low amount per correct sum.

- The right choices correspond to Individual Pay. Under Individual Pay your earnings in Task 1 depend on your performance and on chance. Specifically, you earn the high amount per correct sum with some probability X [a number between 1 and 100]. To determine your earnings, you will throw two ten-sided dice to randomly generate a number between 1 and 100. If the number you generate is lower than the probability $\mathrm{X}$ then you earn the high amount per correct sum, otherwise you earn the low amount per correct sum.

You must decide in every row whether you prefer Individual Pay or Competitive Pay. 
Notice that in a decision set, the high and low amounts for Competitive Pay are the same in all rows. In some decision sets, what varies from row to row is the probability of getting the high amount in Individual Pay. In other decision sets, what varies from row to row is the high amount in Individual Pay.

At the end of the experiment, one of the 8 decision sets will be randomly selected. Within the selected decision set, one of the 10 rows will be randomly selected. The type of payment you chose in the selected row will be used to determine how much you will receive per correct sum in Task 1.

\section{Example 1}

Take a look at the choices in the screenshot below. Now, imagine that this decision set is randomly selected for payment and within this decision set, row number 4 is randomly selected. Given that Individual Pay was chosen instead of a Competitive Pay in this row, then:

- With $27 \%$ of chance, you will earn $€ 5$ per correct sum in Task 1 [the high amount].

- With $73 \%$ of chance, you will earn $€ 0$ per correct sum in Task 1 [the low amount].

\begin{tabular}{|c|c|c|}
\hline & Competitive Pay & Individual Pay \\
\hline 1. & $C 6.00$ if you win and $C 0.00$ if you lose & of $\ell 6.00$ with $21 \%$ chance and $\ell 0.00$ with $79 \%$ chance \\
\hline 2. & C 6.00 if you win and $C 0.00$ if you lose & of $\ell 6.00$ with $23 \%$ chance and $€ 0.00$ with $77 \%$ chance \\
\hline 3. & 66.00 if you win and $\ell 0.00$ if you lose & $c$ \& $\epsilon 6.00$ with $25 \%$ chance and $\epsilon 0.00$ with $75 \%$ chance \\
\hline 4. & 66.00 if you win and $\mathcal{C} 0.00$ if you lose & $c$ \& $€ 6.00$ with $27 \%$ chance and $€ 0.00$ with $73 \%$ chance \\
\hline 5. & $\epsilon 6.00$ if you win and $\epsilon .00$ if you lose & $c$ \& $€ 6.00$ with $29 \%$ chance and $€ 0.00$ with $71 \%$ chance \\
\hline 6. & $\epsilon 6.00$ if you win and $\epsilon .00$ if you lose & $c$ \& $€ 6.00$ with $31 \%$ chance and $€ 0.00$ with $69 \%$ chance \\
\hline 7. & $\epsilon 6.00$ if you win and $\epsilon 0.00$ if you lose & $c$. $\epsilon 6.00$ with $33 \%$ chance and $€ 0.00$ with $67 \%$ chance \\
\hline 8. & $\epsilon 6.00$ if you win and $\epsilon .00$ if you lose & $c$. $\epsilon 6.00$ with $35 \%$ chance and $\epsilon 0.00$ with $65 \%$ chance \\
\hline 9. & $\epsilon 6.00$ if you win and $\epsilon 0.00$ if you lose & C \& 6.00 with $37 \%$ chance and $\ell 0.00$ with $63 \%$ chance \\
\hline 10. & $\epsilon 6.00$ if you win and $€ 0.00$ if you lose & $c$ \& $\ell 6.00$ with $39 \%$ chance and $\ell 0.00$ with $61 \%$ chance \\
\hline
\end{tabular}

Now, imagine that instead of row number 4, the row randomly selected for payment is row number 2. Given that Competitive Pay was chosen instead of Individual Pay in this row, then:

- If you are the group's winner in Task 1 , you earn $€ 6$ per correct sum [the high amount].

- If you are one of the group's losers in Task 1 , you earn $€ 0$ per correct sum [the low amount]. 


\section{TASK 3 - both group sizes}

In Task 3 you will be perform again the same summation task you performed in Task 1 . The main difference is that you will be randomly assigned to a group of six[three] participants instead of three.

\section{TASK 4}

Task 4 is like Task 2 . In Task 4 you can earn money by answering the following question:

How likely do you think it is that you are the winner of your group in Task 3 ?

Again, your will be able to select your answer by moving the cursors in two different sliders:

- In the black slider, you can select your likelihood of being the winner of your group. Your answer can go from 0\% (meaning you are completely certain that you are not the winner of your group) to 100\% (meaning you are completely certain that you are the winner of your group).

- In the green slider, you can select how your performance in task 3 ranks compared to the performance of all participants.

Your answer can go from Top 100\% (you performed worse than ALL other participants of the study) to Top 0\% (you performed better than ALL other participants in the study).

Your earnings in Task 4 will be calculated using the same formulas as in Task 2. Recall that you will obtain the highest expected earnings if your answer equals the actual likelihood of you being the winner in Task 3 .

One important consideration for Task 4, is that to be the winner in Task 3, you need to be the best in a group of SIX[THREE]. The table provided in the next page displays the likelihood of being your group's winner in Task 3 depending on each possible rank. Logically, it is easier[harder] to be the winner in a group of three[six] than in a group of six[three]. This is why the percentages in the table for Task 4 are lower[higher] than the percentages in the table for Task 2.

[Ranking table for the corresponding group size - see page ** for a group size of 3 and page $* *$ for a group size of 6 ] 


\section{TASK 5 - both group sizes}

This is Task 5 of the experiment. The earnings from this part of the experiment are completely independent from the other tasks. The amount you earn depends solely on your decisions and on chance. Moreover, you will not perform further summation tasks.

You will be asked to make choices in 4 different decision tables. All these decision tables are completely independent of each other. An example of one decision table is displayed in the screenshot below.

\begin{tabular}{|c|c|c|}
\hline & Option A & Option B \\
\hline 1. & $€ 28.80$ with certainty & $\ulcorner\subset € 72.00$ with $50 \%$ chance and $€ 0.00$ with $50 \%$ chance \\
\hline 2. & $€ 30.24$ with certainty & $\ulcorner\subset € 72.00$ with $50 \%$ chance and $€ 0.00$ with $50 \%$ chance \\
\hline 3. & $€ 31.68$ with certainty & $\ulcorner\subset € 72.00$ with $50 \%$ chance and $€ 0.00$ with $50 \%$ chance \\
\hline 4. & $€ 33.12$ with certainty & $\ulcorner\subset \in 72.00$ with $50 \%$ chance and $€ 0.00$ with $50 \%$ chance \\
\hline 5. & $€ 34.56$ with certainty & $\sim \subset € 72.00$ with $50 \%$ chance and $€ 0.00$ with $50 \%$ chance \\
\hline 6. & $€ 36.00$ with certainty & $\subset \subset \in 72.00$ with $50 \%$ chance and $€ 0.00$ with $50 \%$ chance \\
\hline 7. & $€ 37.44$ with certainty & $\sim \subset € 72.00$ with $50 \%$ chance and $€ 0.00$ with $50 \%$ chance \\
\hline 8. & $€ 38.88$ with certainty & $\ulcorner\subset \in 72.00$ with $50 \%$ chance and $€ 0.00$ with $50 \%$ chance \\
\hline 9. & $€ 40.32$ with certainty & $\ulcorner\subset € 72.00$ with $50 \%$ chance and $€ 0.00$ with $50 \%$ chance \\
\hline 10. & C 41.76 with certainty & $\subset \subset \in 72.00$ with $50 \%$ chance and $€ 0.00$ with $50 \%$ chance \\
\hline
\end{tabular}

Each table has 10 different decisions, each in a different row. Each decision has two options:

- Option A, where you can earn a different certain amount in each of the 8 rows.

- Option B, where you can earn a high amount with some probability and a low amount with some other probability. Specifically, you earn the high amount with some probability X [a number between 1 and 100]. To determine your earnings, you will throw two ten-sided dice to randomly generate a number between 1 and 100. If the number you generate is lower than the probability $\mathrm{X}$ then you earn the high amount, otherwise you earn the low amount.

You can decide for every row whether you prefer Option A or option B. Option A is the same for every row, while option B takes 8 different amounts, one for each row.

At the end of the experiment, one of the 4 decision tables will be randomly selected. Within the selected table, one of the 10 rows will be randomly selected. The choice you made in that row will determine your earnings of Task 5. 


\section{Chapter 3}

\section{Estimating Preferences for Competition from Convex Budget Sets}

The literature in experimental economics has linked the laboratory measurement of preferences for competition with labor market outcomes and educational choices, such as wages and career choices. If preferences for competition are an important determinant of behavior, it is crucial to develop an accurate approach to measure them. In this study, we test whether preferences for competition can be rationalized by a utility function and develop a framework for the joint treatment of preferences for competition and risk. Our design improves on previous work in that it generates a rich data set of individual-level choices, accounts structurally for the relation between risk and competition preferences, and controls carefully for overconfidence. This allows us to test whether choices to enter tournaments are consistent with GARP and to estimate with a high degree of certainty the extent to which these choices are explained by a preference for competition and not by other confounding factors present in the environment. Our findings provide strong evidence for a preference for competition at the individual level that largely satisfies GARP, and that is conceptualized by two terms in the utility function of income obtained in a competitive environment. The first term assumes that preferences for competition affect utility directly through payoffs and the second term allows preferences for competition to influence utility through risk preferences. We discuss the economic implications of both interpretations of preferences for competition and discuss their relation with risk preferences.

This chapter is coauthored with Ernesto Reuben 


\section{Introduction}

Psychological traits and preferences explain and cause important life outcomes like wages and health (Heckman et al., 2019). Recently, the literature in experimental economics has shown that preferences for competition predict important labor market outcomes and educational choices (Buser et al., 2014; Berge et al., 2015; Reuben et al., 2015; Buser et al., 2017a,b; Reuben et al., 2017; Kamas and Preston, 2018; Zhang, 2019; Buser et al., 2020). Given the important role of preferences for competition in determining behavior, there is a need for an accurate approach to capture them to understand individual choices in a competitive context. We contribute to this endeavor by providing the first test of rationality of individual preferences for competition, and propose a full structural estimation to seize the effect of these preferences on the utility of income derived in a competitive environment.

Since the seminal paper by Niederle and Vesterlund (2007), an individual's preference for competition is typically measured using the choice between two remuneration schemes in a real-effort task: an individual scheme and a tournament scheme. The former depends solely on individual performance, whereas the latter is considered competitive as it depends on individual performance relative to others. This laboratory approach has been widely used by researchers in experimental economics and psychology as it allows capturing preferences for competition in an incentive-compatible way while controlling for confounding factors (for reviews, see, e.g., Niederle, 2014; Dariel et al., 2017). However, recent evidence has questioned the interpretation of such preferences for competition, especially in the presence of risk attitudes (Gillen et al., 2019; van Veldhuizen, 2017). That is, when formalizing preferences for competition into a utility framework, it seems unclear how preferences for competition influence the utility of income derived in a competitive environment versus in a non-competitive environment, and the role that risk preferences have on it.

In this study, we propose two interpretations in which preferences for competition can affect utility. The first one derives from the classical approach in the literature, where a regression-based method is used to capture preferences for competition (Gneezy et al., 2003; Niederle and Vesterlund, 2007; Dariel et al., 2017; Buser et al., 2020). This first interpretation implicitly considers that preferences for competition affect the utility directly through payoffs and conceptualizes such preferences as an additive term in the utility function, independent of risk preferences. In particular, under this interpretation, a competition aversion person will get a dis-utility if her payoffs depend on competition, whereas a competition-seeking person will get a utility from this dependency as she derives pleasure from being in a competitive environment itself. This first interpretation ignores the possibility of the perception of risk to change with the environment, as it has been recently suggested by Abdellaoui et al. (2007), Andersen et al. (2008) Kettlewell (2019), and Schildberg-Hörisch (2018). We address this concern in our second interpretation by allowing for an effect of competition preferences on risk preferences. Specifically, preferences for competition affect the utility 
not only through payoffs as in the first interpretation but also through the effect that preferences for competition have on risk preferences. Specifically, we estimate both preference parameters jointly by conceptualizing preferences for competition and risk as curvature terms in the utility function. ${ }^{1}$ Following this second interpretation, preferences for competition affect the utility derived of income in a competitive environment as they can make someone more or less tolerant to risk.

We propose an experimental approach that allows testing these two interpretations. By introducing changes at the individual level in both the tournament prize and piece-rate prize simultaneously, we generate a rich data set suitable to test for consistency of individual choices between a competitive and non-competitive environment, and to map such individual choices into a utility function that captures preferences for competition. To the best of our knowledge, all studies using the design proposed by Niederle and Vesterlund (2007) rely on a single individual choice between the two payment-schemes as their measurement for preferences for competition. We argue that this approach can be problematic when deriving preferences as oftentimes individual choices can be noisy. Evidence suggests that individuals make different choices when confronted several times with the same set of options (Tversky, 1969; Camerer, 1989; Hey and Orme, 1994; Agranov and Ortoleva, 2017). In addition, it is impossible to check whether individual preferences for competition are consistent when having only one choice per individual. Checking for consistency of individual choices is necessary to rationalize behavior with a utility function, and therefore, to claim the existence of a preference (Afriat, 1972). Extensive literature in economics has shown the advantages of expressing individual choices in economic domains in a "well-behaved" preference ordering (Andreoni and Miller, 2002; Choi et al., 2007; Fisman et al., 2007; Andreoni and Sprenger, 2012). This literature applies the axioms of revealed preferences - such as the Generalized Axiom of Revealed Preference (GARP) - to infer the existence of a convex, continuous, and monotonic preference ordering and to estimate preferences using individual choices.

In our design, we implement a variation of the experimental design proposed by Niederle and Vesterlund (2007) to study whether individual choices between a competitive and non-competitive environment can be rationalized with a utility function, and therefore, be represented by preferences for competition within our two interpretations. In the task, participants choose between a tournament rate and an individual rate as remuneration for their future performance in a real effort task. Similar to Niederle and Vesterlund (2007), the individual rate represents the

\footnotetext{
${ }^{1}$ As suggested by Andersen et al. (2008) neglecting the structural relation between preferences in different domains can lead to biased estimates. For instance, for the case of risk and time preferences, joint estimation of preferences is necessary to avoid "curvature bias" in the estimates (Andersen et al., 2008). Specifically, not representing the preferences of these two different domains by the curvature of the utility function leads to biased estimates of individual discount rates. Abdellaoui et al. (2007) suggests a similar pattern for the case of loss aversion and risk preferences, where the risk parameter might be biased due to ignoring loss aversion.
} 
non-competitive environment as it depends only on individual performance whereas the competitive rate recreates a competitive environment as depends on relative performance. However, differently to the design proposed by Niederle and Vesterlund (2007), we implement the choice between the two remuneration schemes as a choice from a convex budget set (CBS), similar in spirit to the approach proposed by Andreoni and Miller (2002) to study the consistency of choices and individual preferences. Lastly, after the selection of the payment-scheme for future performance, we elicit individual beliefs about relative performance and risk preferences; this allows to control for the role of overconfidence and risk attitudes on shaping individuals' preferences for competition.

Our design offers a number of benefits for the estimation of preferences for competition. First, it facilitates the test of consistency of individual choices between a competitive and non-competitive environment. Testing for consistency is crucial as it provides enough evidence of a utility-maximizing behavior, which is necessary to claim the existence of a preference for competition (Afriat, 1972). In particular, the theory of revealed preferences proposes an exact test for consistency with the GARP. Following the approach proposed by Afriat (1972), we capture the extent of GARP violations with the critical cost efficiency index (CCEI) to measure consistency of preferences in a competitive environment. Second, our design allows us to account for preferences for competition across a wider array of environments than the classical approach proposed by Niederle and Vesterlund (2007). This is possible as we expose participants to several prizes with the help of the CBS setting (i.e., 40 different individual and tournament rates are changed at the individual level). ${ }^{2}$ Third, it provides more information about preferences than a discrete choice setting (Andreoni and Miller, 2002). Fourth, the rich data set is suitable for estimating preferences at the individual level without the need to aggregate participants together and to assume homogeneity across them (Fisman et al., 2007). Lastly, using a structural estimation approach allows to evaluating jointly preferences for competition and risk, and their covariates. This approach has the advantage to capture the structural dependencies between the preferences parameters Abdellaoui et al. (2007); Andersen et al. (2008); Meissner et al. (2020) and it allows us to estimate the preferences parameters as a linear function of individual characteristics simultaneously accounting for individual heterogeneity in both a risky and a competitive domain (Harrison et al., 2007).

Our main findings reveal the following. First, we observe that most of the individual choices between a competitive and non-competitive environment satisfy the GARP axiom, and can thus be rationalized by a utility function (i.e., 94\% of our participants are consistent as their choices do not violate GARP). Second, individual choices in a competitive environment are captured by a preference for competition with the economic models we derive from our two

\footnotetext{
${ }^{2}$ As discussed in detail Section 2, only few studies in competitive behavior have introduced changes in the stakes (i.e., prizes under the individual or tournament rates) (Petrie and Segal, 2015; Flory et al., 2015; Ifcher and Zarghamee, 2016).
} 
interpretations. This suggests that preferences for competition can be represented in a utility function both as an additive and curvature term. Meaning that competition has a direct effect on payoffs but also on risk attitudes. Third, the estimates from our two economic models suggest that participants are on average risk averse and have a general dislike for competition that is captured by the additive term in our model. Interestingly, participants display less risk aversion behavior once we allow for an effect of preferences for competition on risk. That is, when we account for the structural relation between risk and competitiveness simultaneously, and model preferences for competition as a curvature term in the utility function. This finding suggests that risk preferences differ between an environment with and without competition, and ignoring this relation might overestimate the level of risk aversion in the population. In fact, this is what happens with our first interpretation where preferences for competition are only represented as an additive term. In addition, our findings also suggest that preferences for competition and risk are defined for most individuals as we detect small heterogeneity in competitive and risk aversion parameters. Lastly, in line with the literature in experimental economics, we show that gender differences in competition are persistent when capturing preferences for competition as an additive term (Gneezy et al., 2003; Booth and Nolen, 2012; Dariel et al., 2017; Saccardo et al., 2018). However, once we account for the effect of preferences for competition on risk and capture preferences for competition as curvature term, gender differences in preferences for competition disappear as suggested by Gillen et al. (2019); van Veldhuizen (2017).

The remainder of this chapter is as follows. In Section 2 we provide a literature review. Section 3 describes our experimental design and procedures. In Section 4, we present our results for consistency and our measurement of preferences for competition represented by our two economic models. In Section 5 , we discuss our results and conclude.

\section{Literature review}

This paper contributes to the discussion on preferences for competition and on how to measure them in the laboratory. Since with the seminal paper of Niederle and Vesterlund (2007) (henceforth NV), there have been many attempts in trying to capture willingness to compete, especially in the context of gender differences (Gneezy et al., 2003; Booth and Nolen, 2012; Dariel et al., 2017; Saccardo et al., 2018). But how have these preferences for competition been measured so far? In the classical experimental task of $\mathrm{NV}$, an individual's preference for competition is typically captured using a single choice between two remuneration schemes when doing a real-effort task. Specifically, participants can choose between two different schemes: a piece-rate, that depends only on individual performance; and a tournament rate, that depends on relative performance. The tournament option is considered a competitive scheme as it requires participants to compete against each other. With the help of a regression-based approach and after controlling for individual's overconfidence, risk preferences, and ability, the choice 
between the two remuneration schemes is considered as individual's preference for competition. ${ }^{3}$

The vast majority of studies using the $\mathrm{NV}$ design rely on a regression-based method to measure preferences for competition, whose accuracy depends strongly on the ability to control successfully for all the other determinants present in a competitive environment. Hence, a measurement error or misspecification in any of these determinants can bias the interpretation of the results (Hausman, 2001; Green et al., 2010; Westfall and Yarkoni, 2016; Gillen et al., 2019). Two studies that analyze gender differences in the preferences for competition propose a different approach. Gillen et al. (2019) develop a statistical technique to correct for measurement error in the risk attitudes and overconfidence measures used in the NV design. Their findings suggest that after accounting for the measurement errors of these two traits, the role of preferences for competition in explaining the gender gap in competition disappears. That is, they suggest that only risk and overconfidence matter when explaining the gender gap in competition. In another study, van Veldhuizen (2017) proposes a modified version of $\mathrm{NV}$ to differentiate by design between risk attitudes, overconfidence, and preferences for competition. In particular, his design introduces treatments that remove sequentially the role of competition and overconfidence and compares these treatments with the classical NV outcome. In contrast to most of the previous studies on competition, his findings suggest that the gender gap in competition is mainly captured by gender differences in risk preferences and overconfidence. $^{4}$ We contribute to the discussion on how to measure preferences for competition by developing a different approach to the ones proposed by Gillen et al. (2019) and van Veldhuizen (2017). Specifically, we allow for an effect of preferences for competition on risk preferences and capture such a relation structurally. In addition, we do not rely on one single individual choice, but we capture individual preferences for competition by confronting individuals with a wide range of competitive scenarios generated with the CBS setting.

To the best of our knowledge, only a few studies have introduced changes in the incentives in the competitive environment. In a laboratory experiment, Ifcher and Zarghamee (2016) introduce a continuous measurement of willingness to compete to identify the minimum piece-rate payment that individuals prefer to a tournament payment. In their design, the piece-rate prize changes while the tournament prize is always the same. Their findings suggest that men are more willing to compete than women for specific tournament prize ranges. In another lab study, Petrie and Segal (2015)

\footnotetext{
${ }^{3}$ Other factors associated to individual heterogeneity in a competitive environment are differences in ambiguity attitudes and feedback aversion (Wozniak et al., 2016; Ertac and Szentes, 2011; Friedl et al., 2017). In our study, we do not target these factors since they do not take place in our experimental setting or they are inherently present in a competitive environment as in the case of ambiguity.

${ }^{4}$ For a different approach on how to control risk attitudes by design refer to Geraldes (2020). His approach is different from ours because when controlling for risk by design he uses individuals that self-selected into the non-competitive payment whereas we use those that had to perform under a competitive environment.
} 
investigate whether changes in the tournament prizes affect the gender gap in selecting into tournament. Their findings suggest that for a sufficiently high prize women choose to compete as much as men, closing the gender gap in tournament entry. However, similar to NV, in both studies, they use one single choice as their measurement for competition. In addition, the authors introduce changes in either the tournament or the piece-rate prizes but not in both prizes which can be more informative to derive preferences for competition. In a natural field experiment, Flory et al. (2015) implement tournament with changes in the payment scheme (making it less or more competitive) and examine gender differences in the application rates for a job advertisement. Their findings reveal that fewer women apply if the payment scheme is a competitive one (i.e., based on relative performance) or is uncertain, compared to a flat hourly wage payment scheme. Our approaches differ as we are interested in measuring preferences for competition instead of studying job application rates. ${ }^{5}$

\section{Experimental design and procedures}

Participants are invited to a lab session to make choices in an incentivized experiment. In the following two subsections, we describe the experimental design where we implement a risky and a competitive environment, and the experimental procedures, respectively.

\subsection{Experimental design}

We propose a variation of the experimental design by $\mathrm{NV}$ to measure participant's willingness to compete and to generate a rich data set at the individual level suitable to estimate preferences. Participants perform an adding task under a selected payment-scheme: Individual Rate or Tournament Rate. We use participants' choice of the payment-scheme as our measurement for their taste for competition. We introduce two novel key features in our design. First, participants face a convex combination of the two payment-schemes instead of the discrete choice in the classical NV setting. Second. participants do not make a single choice between the two schemes, but they are confronted with several decision sets where we vary the Tournament and Individual Rates. To apply these changes to the original design by NV, we follow the approach developed by Andreoni and Miller (2002) to implement the choice between the two payment-schemes as a choice in a convex budget set (CBS) in 40 different decision situations.

Each experimental session is divided into five parts which are described in detail below. In the first two parts, participants perform the adding task first under an Individual Rate scheme and then under a Tournament Rate scheme.

\footnotetext{
${ }^{5}$ Another way of introducing changes in incentives has been done through changes in the number of winners, and therefore, by introducing changes in the expected returns from winning the tournament (Freeman and Gelber, 2010). However, it is hard to compare their setting with ours because in (Freeman and Gelber, 2010) the main variable of interest is the amount of effort exerted by participants and not preferences for competition itself.
} 
In Part 3, participants choose between these two different payment-schemes for their future performance in 40 different CBSs. In Part 4, we elicit participants' beliefs of being the best performer in the adding task. In Part 5, we elicit individual risk preferences following the same setting used in Part 3. Lastly, participants complete a demographic questionnaire about demographic characteristics such as gender, age, number of siblings and position among them, nationality, and study level. The instructions for each part are provided at the beginning of the respective part and can be found in Appendix A5.

Importantly, although participants know their own absolute performance, they are never informed about the performance of others in the adding task. ${ }^{6}$ Relative performance is only revealed at the end of the experiment if the Tournament Rate is selected for payment purposes. ${ }^{7}$

\section{Parts 1 and 2 - Performance under Individual and Tournament rates}

At the beginning of the experiment, participants perform a four-minute adding task first under an Individual Rate and later under a Tournament Rate. This with the aim to get familiarized with the two different payment-schemes before the selection of one payment-schemes for future performance. The adding task consists in adding-up sets of four two-digit numbers during four minutes. The numbers are randomly drawn by the computer from a uniform distribution with a support of 1 to 99. Participants are not allowed to use a calculator, but they are provided a scratch paper. Every time they submit an answer, the computer immediately informs them whether the answer is correct or incorrect and a new sum is generated. In addition, the computer keeps a record of the number of correct sums.

Under the Individual Rate, the payoffs depend only on individual performance. That is, participants receive 100 tokens per correctly answered sum. ${ }^{8}$ Under the Tournament Rate, the payoffs depend on participants' relative performance. Specifically, participants are randomly assigned to a group of five people. The participant who correctly solves the highest number

\footnotetext{
${ }^{6}$ Note that this feature creates naturally ambiguity in the tournament as in the tournament payment-scheme participants do not know their relative performance. This is the ambiguity that is usually inherently present in a competitive environment and can be solved by providing feedback on relative performance as proposed by Wozniak et al. (2016). Another way in which ambiguity is present in a tournament is by not knowing the number of competitors as recently suggested by Flory et al. (2015), Balafoutas and Sutter (2019) and Gee (2019). In our setting, the number of competitors is always common knowledge.

${ }^{7}$ Although this feature introduces differences in feedback between the two paymentschemes, feedback on relative performance is one of the key differences between a competitive environment and a non-competitive environment. Also, we believe this is not a concern in our design for two reasons. First, participants never received explicit information about feedback being revealed only if the Tournament Rate was selected for payment purposes. Therefore, participants could not anticipate feedback under only the Tournament rate. Second, although participants still could have beliefs on differences in feedback provision between the two schemes, previous evidence suggests that feedback aversion does not play a role in a setting similar to ours (Niederle and Vesterlund, 2007).

${ }^{8}$ Participant know that the number of tokens is transformed into Euros at the exchange rate 100 tokens $=€ 1,00$
} 
of sums in their group is the group's winner. In case of any tie, the winner is determined randomly among the tied group members. The earnings under a Tournament Rate are as follows: the group winner receives 550 tokens per correctly answered sum while everyone else in the group receives 0 tokens.

\section{Part 3 - Choice of payment-scheme for future performance}

Before performing the adding task, participants choose how they want to be paid for each correct sum in a future task. Specifically, they can choose a combination of Individual Rate and Tournament Rate in 40 different decision sets that are randomly generated at the individual level. Each decision set consists of a budget line that contains combinations of the Individual and Tournament Rates. Figure 3.1 displays an example of a decision set where the Individual Rate corresponds to the horizontal axis, and the Tournament Rate corresponds to the vertical axis. To make a choice between the two payment-schemes participants can pick a point on the budget line, where every point corresponds to a different combination of Individual Rate and Tournament Rate. ${ }^{9}$

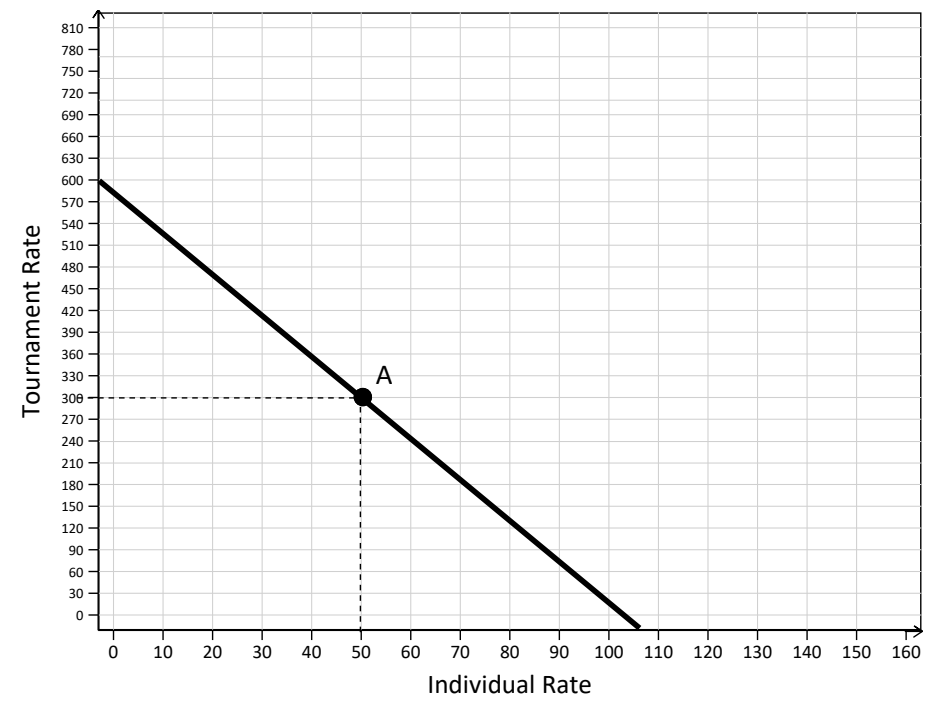

Figure 3.1: Example of a budget line for the choice of the payment-scheme

The Individual Rate pays the rate on the horizontal axis that corresponds to the point they choose on the budget line. For instance, as displayed in Figure 3.1, if a participant chooses a point A on the budget line, the Individual Rate is 50 and she will receive 50 tokens per correct sum under the individual rate. Similarly, the Tournament Rate pays the rate on the vertical axis that corresponds to the point they choose on the budget line if they are the winner of their group in Part 3. For example, as shown in Figure 3.1, if a participant chooses point A on the budget line, the Tournament Rate is 300 and she will

\footnotetext{
${ }^{9}$ See Appendix A5 Figure 3.24 for a real example of the screen that was displayed to participants in this part.
} 
receive 300 tokens per correct sum if she is the group's winner, and 0 tokens otherwise. Participants' performance in Part 3 is compared with the performance of the four other members of their group in Part 2, where they perform under the Tournament rate. This ensures that participants' choices in Part 3 do not create externalities in the payoffs of the other participants (Niederle and Vesterlund, 2007). A participant is the group winner if she solves more sums in Part 3 than all the other group members in Part 2. If there are ties, the winner is randomly determined among the tied group members. If she is not the group winner, she receives 0 tokens. The final earnings in Part 3 are determined as follows: If a participant is not the winner of the tournament, then Earnings $=$ (Individual Rate $*$ correct sums $)$. If a participant is the winner of the tournament: Earnings $=($ Individual Rate + Tournament Rate) * Correct sums. To help participants to understand the consequences of their choices in Part 3, we use an interactive interface: for each point selected on the budget line, participants could see on the screen instantaneously both the rates corresponding to the selected point and the specific payoffs in tokens associated with their selection for both cases, being the winner and not being the winner. ${ }^{10}$

Participants make a choice in 40 different decision sets with a different budget line in each set. Following the design of Fisman et al. (2007), we generate different budget lines at the individual level by randomizing the intersection point of the budget line with the horizontal axis between any value ranging from 50 to 150 tokens and the intersection point of the budget line with the vertical axis between any value ranging from 300 to 800 tokens. As suggested by Fisman et al. (2007), this ensures that there is enough variation in the slopes of the different budget sets which is crucial to test the rationality of individual choices, and consequently, to estimate preferences. This feature of Part 3 allows us to structurally estimate a parameter of preferences for competition at the individual level. ${ }^{11}$ After participants make a choice in all 40 budget lines, one of the lines is randomly selected with equal probability to be relevant for the determination of the earnings in Part 3. Afterward, participants perform the adding task for Part 3 knowing the Individual Rate and Tournament Rate that determine their earnings in this part.

\section{Task 4 - Beliefs of future performance}

Before performing the adding task under the selected payment-scheme in Part 3 , participants guess the likelihood of being the winner in their group in Part 3 (i.e., the probability of having the highest amount of correct sums in their group when compared to the performance of players in Part 2). This task helps us to derive a measure of overconfidence that will be later used in the estimation of individuals' preferences for competition.

\footnotetext{
${ }^{10}$ See Appendix A5 Figure 3.23 for an example of the screen with the interactive interface.

${ }^{11}$ For a summary of the intercepts with the $\mathrm{x}$ and $\mathrm{y}$-axis, please refer to Appendix A1, Table 3.6.
} 
In Part 4, participants can choose an answer ranging from 0 (i.e., they are completely certain that they are not the winner of their group) to 100 (i.e., they are completely certain that they are the winner of their group). The earnings in this part can be either $€ 0$ or $€ 20$. We incentivize beliefs using a robust binarized scoring rule (BSR) (Karni, 2009). In particular, given a stated likelihood of being the winner, belief, the BSR incentive offers a $1-(1 \text { - belief } / 100)^{2}$ chance of earning $€ 20$ in case of being the winner, and a 1 - (belief $/ 100)^{2}$ chance of earning $€ 20$ in case of being one of the losers. Thus, the closer the likelihood the participants choose is to the actual probability of wining the tournament, the higher the probability of earning $€ 20$. To increase the chances of true reporting, we provide participants with information concerning the quantitative incentives in the elicitation method. ${ }^{12}$ In particular, using an interactive interface participants could see on the screen instantaneously the expected earnings in euros associated with any selected likelihood. ${ }^{13}$

\section{Part 5 - Risk Preferences elicitation}

We elicit participants' risk preferences with a similar setting to the one used in Part 3. Participants choose combinations of a Certain Amount and a Probabilistic Amount in 40 different decision sets that are independent of each other. Each decision set contains a budget line where the Certain Amount corresponds to the horizontal axis and the Probabilistic Amount corresponds to the vertical axis as displayed in Figure 3.2. Every point on the budget line resembles a combination of a Certain Amount and a Probabilistic Amount. Participants can only choose one point on the budget line, but they are free to choose any point. In Part 5 participants do not have to perform any adding task as in Part 3.

The Certain Amount pays the amount on the horizontal axis that corresponds to the point they choose on the budget line for sure. For example, as displayed in Figure 3.2 if a participant chooses point B on the budget line, the Certain Amount is 500 and she receives 500 tokens for sure. The Probabilistic Amount pays the amount on the vertical axis that corresponds to the point they choose on the budget line with a probability of $20 \%$, and 0 with a probability of $80 \%$. For instance, if a participant chooses point B, the Probabilistic Amount is 2400 and she receives 2400 tokens with $20 \%$ chance and 0 tokens with $80 \%$ chance. These probabilities are chosen to resemble the

\footnotetext{
${ }^{12}$ In a recent study, (Danz et al., 2020) shows that providing precise information on the BSR quantitative incentives can influence the truth-telling over an objective prior (Danz et al., 2020). Although Danz et al. (2020) findings suggest that the false reports in beliefs are due to thinking in terms of probabilities, it is not entirely clear this will apply as well to expected earnings. We believe that in our experiment this is not a concern given that even though we provide details about the quantitative incentives, participants are only informed about the consequences of their selected beliefs in terms of expected earnings and not in terms of the probability of winning the prize.

${ }^{13}$ In Appendix A5, Figure 3.28 is displayed the actual screens that participants see during the experiment for the belief elicitation task.
} 


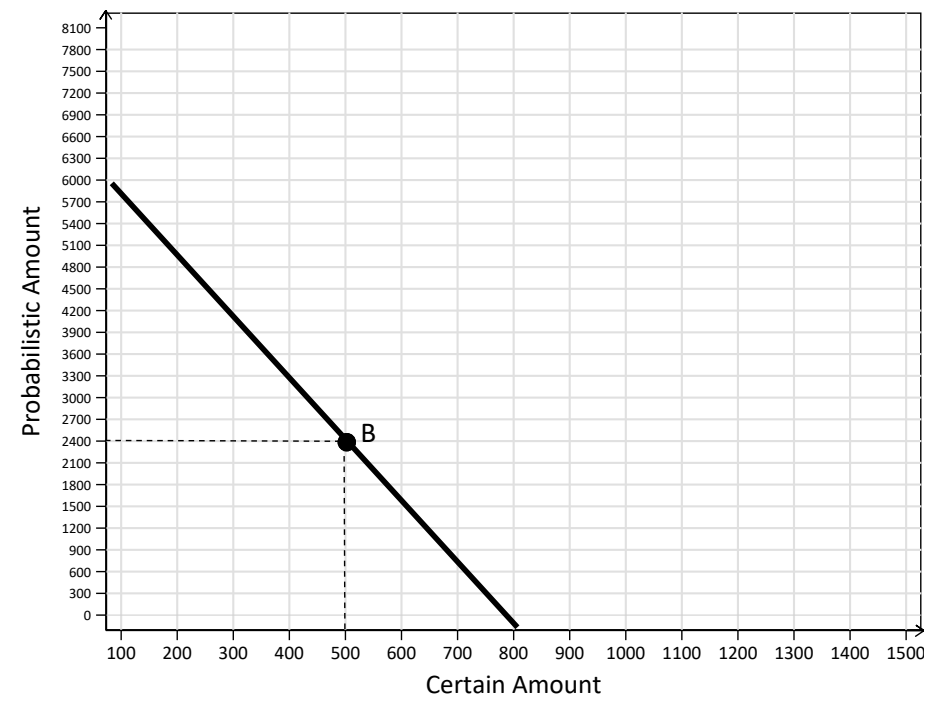

Figure 3.2: Example of a budget line for the choice between the Certain Amount and the Probabilistic Amount.

probability of being the winner in a group of five people as in Part $3 .^{14}$

To resemble the incentives in Part 3, the final earnings in Part 5 are determined as follows: with a probability of $80 \%$ a participant receives her selected Certain Amount, and with a probability of $20 \%$ she receives her chosen Certain Amount plus her chosen Probabilistic Amount. Note that this ensures that the Certain Amount is always received, regardless of the two probability scenarios. Participants make a choice in 40 different decision sets with a different budget line in each set. Similar to Part 3, in each decision set the computer randomly generates a budget line that crosses the horizontal axis between 500 and 1500 tokens and the vertical axis between 3000 and 8000 tokens. ${ }^{15}$ Similar to Part 3, this creates enough variation in the slopes of the different budget sets presented in Part 5. This will help us to estimate individual risk preferences and also to test for consistency of individual choices between a risky and non-risky environment. In addition, the measurement of risk preferences derived from this part will be used to estimate preferences for competition in the presence of risk preferences. ${ }^{16}$ After participants make a choice for all the 40 lines, one of them is randomly selected with equal probability to determine their earnings for Part 5.

\footnotetext{
${ }^{14}$ Figure 3.30 in Appendix A5 displays the actual screens that participants see during this part of the experiment.

${ }^{15}$ Note that the axis values in Part 5 correspond to the ones used in Part 3 times 10. This is done with the purpose of making comparable the choices from Part 3 and Part 5, as it adjusts the values from Part 3 with the average performance of 10 sums in the adding Task.

${ }^{16}$ See Appendix A1, Table 3.6 for a summary of the intercepts with the $\mathrm{x}$ and $\mathrm{y}$-axis.
} 


\subsection{Experimental procedures}

The experiment was programmed using the software zTree (Fischbacher, 2007) and it was conducted at the Behavioural and Experimental Economics Laboratory (BEElab) at Maastricht University. The experiment consisted of 8 sessions of 20 participants on average each. 140 participants were recruited in total, 77 women and 63 men, through the online recruitment system ORSEE (Greiner, 2015).

Participants signed an informed consent before participating in the study. One of the five parts was selected for payment purposes at the end of each session and this was known by the participants from the beginning. ${ }^{17}$ Participants received $\mathrm{a} € 5$ show-up fee and the total earnings were on average of $€ 25$. Each session lasted on average 1 hour and 45 minutes.

\section{Results}

In this section, we present an exploratory analysis of whether participants have a consistent preference for competition. We start by looking at the consistency of individual choices in our competitive environment, and once we confirm that individual choices are consistent, we check whether such individual choices can be captured by a preference for competition. Specifically, we look at violations of GARP in the choices participants make in Part 3 and Part 5 of our experiment to check for consistency, and verify if individual choices can be rationalized with a utility function that represents preferences for competition and risk. Thereafter, we investigate whether the consistent individual choices between a competitive and non-competitive environment can be captured parametrically by a preference for competition in a structural model.

This section is divided into four parts. First, we provide some descriptive statistics of the performance of our sample in the experimental tasks. Second, we present a consistency test of individuals' choices with GARP. Third, we discuss our two proposed structural models and present the estimates for individuals' preferences for competition. Lastly, we test whether there are gender differences in both our measurements of consistency and preferences for competition.

\subsection{Descriptive statistics}

Table 3.1 displays a summary of participants' performance in the adding task under different payment-schemes, the average amount of tokens allocated to the different payment-schemes under a competitive environment (i.e., Tournament Rate vs. Individual Rate, in Part 3), and a to the different amounts in a risky environment (i.e., Probabilistic Amount vs. Certain Amount, in Part 5), and lastly the average reported belief of being the winner in their group by gender (i.e., Part 4). On average, participants solve correctly

\footnotetext{
${ }^{17}$ See Appendix A1, Table 3.5 for a summary of how the earnings are calculated in each of the five parts.
} 
11 sums under an Individual Rate, 12 sums under Tournament Rate, and 13 sums under the choice of the payment-scheme. This increase in the number of correct sums suggests that there is learning in the adding task, which is consistent with the behavior observed in previous studies using this real effort task (Niederle and Vesterlund, 2007). We observe that women solve on average one correct sum less than men. A two-sample Wilcoxon Rank Sum (Mann-Whitney) non-parametric test reveals that these differences in performance between men and women are significant under the Individual Rate $(p=0.012)$ and choice of the remuneration scheme $(p=0.027)$, but not under the Tournament Rate $(p=0.123) .{ }^{18}$

Table 3.1: Summary of behavior in the experimental tasks

\begin{tabular}{|c|c|c|c|}
\hline & $\begin{array}{c}\text { All } \\
(\mathrm{n}=140)\end{array}$ & $\begin{array}{l}\text { Women } \\
(\mathrm{n}=77)\end{array}$ & $\begin{array}{c}\text { Men } \\
(n=63)\end{array}$ \\
\hline \multicolumn{4}{|l|}{ Performance adding Task } \\
\hline Indv. Rate (\# sums) & $11[3.66]$ & $10.4[3.58]$ & $11.61[3.67]$ \\
\hline Tourn. Rate (\# sums) & $12[4.49]$ & $11.44[4.00]$ & $12.61[4.99]$ \\
\hline \multicolumn{4}{|l|}{ Choice payment-scheme } \\
\hline Performance (\# sums) & $13.1[4.54]$ & $12.2[4.12]$ & 13.9 [4.86] \\
\hline tokens into Tourn. Rate & $207.1[140.88]$ & 169.7 [116.25] & $244.4[157.51]$ \\
\hline tokens into Indv. Rate & $63.8[24.41]$ & $69.2[20.85]$ & $58.5[27.19]$ \\
\hline \multicolumn{4}{|l|}{ Risk preferences } \\
\hline tokens into Prob. amount & $1,883.1[1522.83]$ & $1,352.8[1110.52]$ & $2,413.3$ [1748.95] \\
\hline tokens in Cert. amount & $881.5[439.75]$ & $903.3[376.97]$ & $859.7[508.2]$ \\
\hline \multicolumn{4}{|l|}{ Reported belief } \\
\hline Prob. of being the winner $(\%)$ & 52.4 [26.97] & $50.1[26.60]$ & $54.7[27.42]$ \\
\hline
\end{tabular}

Note: values represent the average performance in the adding task, the average tokens selected under the different payment-schemes, and the average reported belief for each category, i.e., gender and all participants. Standard deviations under [brackets].

Regarding the choice of the payment scheme, Table 3.1 shows that an average of 207 tokens is allocated to the Tournament Rate, whereas an average of 64 is allocated to the Individual Rate. ${ }^{19}$ Two separate Wilcoxon Rank Sum (Mann-Whitney) non-parametric tests show that women allocate less of tokens to the Tournament Rate $(p=0.005)$ and more tokens to the Individual Rate $(p=0.016)$ compared to men. For the risk preferences task in Part 5, an average of 1883 tokens is allocated to the Probabilistic Amount and 882 tokens to the Certain Amount. Two separate Wilcoxon Rank Sum (Mann-Whitney) non-parametric test reveal that women allocate fewer tokens to the Probabilistic Amount compared to men $(p<0.001)$ and that there are no statistically significant differences between men and women for the tokens allocated to the Certain Amount $(p=0.345) .{ }^{20}$

\footnotetext{
${ }^{18}$ Please note that we provide a more in-depth discussion of gender differences on consistency and preferences in Section 4.4.

${ }^{19}$ In Appendix A1, Table 3.6 and Figure 3.8 display the average number of times participants choose a corner choice in Parts 3 and 5 of the experiment.

${ }^{20}$ Note that the discrepancy in differences between the Probabilistic and the Certain Amount is
} 
Finally, the last row in Table 3.1 shows that participants report an average belief of being the winner in their group of $52 \%$. In addition, the average belief for women in our sample is $50 \%$ whereas for men is $55 \%$. However, a twosample Wilcoxon Rank Sum (Mann-Whitney) non-parametric test reveals that this difference between men and women is not significant $(p=0.140)$.

\subsection{Testing rationality}

Before estimating preferences for competition and risk, we investigate whether individual choices in a competitive and risky environment are consistent with the preference ordering of the expected utility theory (EUT). Following the results derived from the theory of the revealed preference proposed by Afriat (1972), we know that a data set of individual choices can be rationalized by a "well-behaved" utility function if these decisions satisfy GARP (i.e., Generalized Axiom of Revealed Preference). ${ }^{21}$ Since individual choices could violate GARP empirically, it is necessary to evaluate to what extent individual choices are consistent with this axiom. In this section, we describe one of the measures that allow us to test the economic rationality of individual choices in a competitive and risky environment.

We check for consistency with the critical cost-efficiency index (CCEI) which captures the minimum adjustments to the budget lines needed to remove violations of GARP (Afriat, 1972). The value of the CCEI ranges from 0 to 1 , where the closer to 1 , the smaller the severity of GARP violations. In the literature, there is no consensus of what constitutes a good threshold to determine whether people's choices sufficiently satisfy GARP. However, some scholars suggested that a reasonable threshold of consistent choices can range between a minimum of 0.8 and 0.95 (Varian et al., 1991; Choi et al., 2007). Figure 3.3 displays the distribution of CCEI scores for both the selection of the remuneration scheme and the risk elicitation tasks in the left and right panel, respectively (i.e., Part 3 and Part 5 in the experiment). The figure shows that the majority of participants (around 94\%) has an index above 0.9 in both the competitive and risky environment. ${ }^{22}$

possible because in the risk elicitation task men have a significantly higher total amount of tokens to allocate than women (mean of total tokens to allocate, for men=1260 and women $=1144$; Two-sample t-test with equal variances $p>0.001$ ). This was the result of randomization of the axis intercepts at the individual level. In our estimation model in Section 4, we account for this issue carefully.

${ }^{21}$ Formally, the GARP axiom states the following: let X, Y be distinct bundles of alternatives, each lying on a linear budget constraint. GARP requires that if $\mathrm{X}$ is (indirectly) revealed preferred to $\mathrm{Y}$, then $\mathrm{Y}$ is not strictly directly revealed preferred to $\mathrm{X}$, that is, $\mathrm{X}$ is not strictly within the budget set when $\mathrm{Y}$ is chosen (Varian, 1982).

${ }^{22}$ In a similar setting like ours, where the different budget lines are randomly generated, Fisman et al. (2007) also observes that most of the subjects in their sample have a CCEI score above 0.9 (around $75 \%$ of their sample). In their design, Fisman et al. (2007) use the test designed by Bronars (1987) to randomize uniformly the choices of a hypothetical subject among all allocations on each budget line as a benchmark and compare the distribution of CCEI scores of subjects in their sample with the CCEI scores of 25,000 hypothetical subjects. The results from this hypothetical sample suggest that the majority of the subjects have a consistent behavior when compared to those generated from random choices. Fisman et al. (2007) conclude that most of the subjects in their sample exhibit an optimizing behavior as it satisfies the GARP. Given 


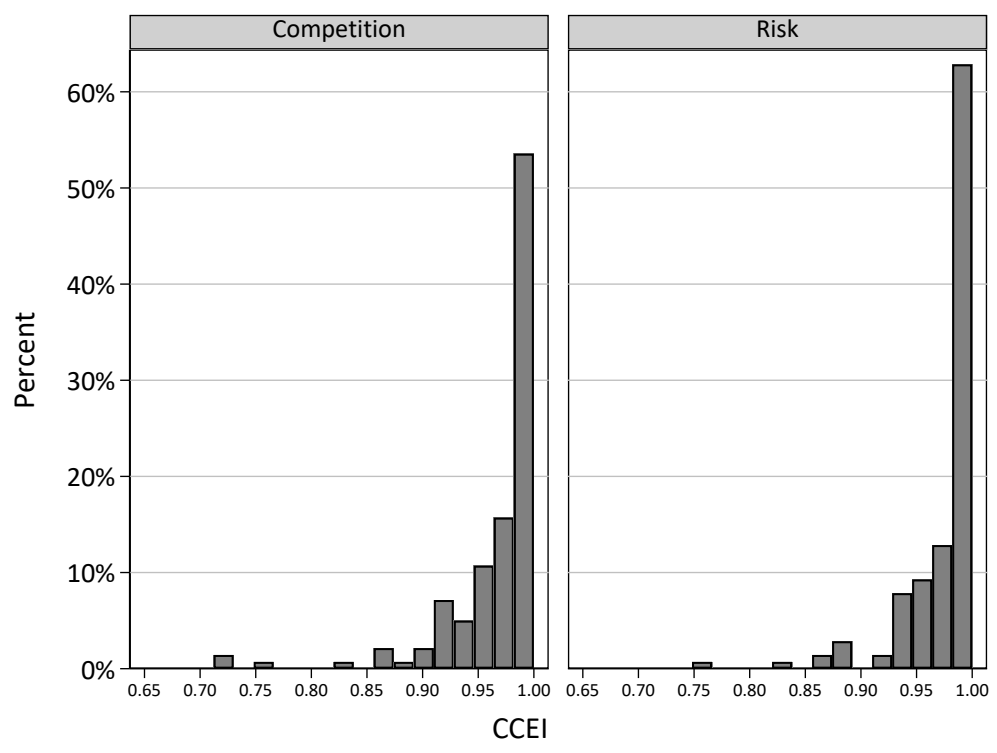

Figure 3.3: Distribution of Afriat's (1972) Critical Cost Index (CCEI) for competitive and risky behavior.

Table 3.2 lists the mean and median of the number of GARP violations and CCEI, as well as the number of subjects that have a CCEI above a threshold of 0.90 and 0.95 for choices in a competitive and risk environment. In the literature, GARP violations have been widely studied in the context of risk preferences but not in the context of preferences for competition. For risk preferences, our data reveal very similar patterns to the ones previously observed (Choi et al., 2007), with an average of 17\% (7 out of 40 budget lines) of the budget lines with a violation of the GARP and a mean CCEI of 0.97. Also, we observe that $94 \%$ and $84 \%$ of the participants have a CCEI above 0.90 and 0.95 , respectively.

For choices in the competitive environment, the results in Table 3.2 suggest a high consistency of individual choices with a mean CCEI of 0.96 . Participants display GARP violations in an average of 8 budget lines out of 40. This number of violations is similar to the one observed in other preference domain settings such as the one of risk preferences, with an average of $20 \%$ of the budget lines displaying GARP violations (Choi et al., 2007; Fisman et al., 2007). Importantly, despite these GARP violations, $94 \%$ and $79 \%$ of the participants have a CCEI above 0.90 and 0.95, respectively. We, therefore, conclude that individual choices in both environments are largely consistent and can be rationalized by a "well-behaved" utility function that captures both competition and risk preferences. It is worth noting that we do not observe significant differences in the number of GARP violations between competitive

that ours is a very similar setting to the one of Fisman et al. (2007) and the distribution of CCEI scores in our sample is even more squeezed towards the right (around 94\% have a CCEI score above 0.9 in comparison to a $75 \%$ in their setting), we infer that the choices of subjects in our sample will also be highly consistent when compared to choices that are randomly generated. 
and risky environments (Kruskal-Wallis Test, $p=0.181$ ). We observe small significant differences in the CCEI between the two environments (Kruskal-Wallis Test, $p=0.042$ ), but not in the proportion of subjects with a CCEI above a threshold of 0.90 and 0.95 (Two-sample test of proportions, $p=1.00$ and $p=0.065$, respectively).

Table 3.2: CCEI for competitive and risky behavior

\begin{tabular}{lcc}
\hline & Competition & Risk \\
\hline Mean (median) \# budget lines with GARP violations & $8(6)$ & $7(5)$ \\
Mean (median) CCEI & $0.966(1)$ & $0.975(1)$ \\
CCEI threshold: & & \\
\# (\%) subjects with CCEI $>0.90$ & $131(94 \%)$ & $131(94 \%)$ \\
\# (\%) subjects with CCEI $>0.95$ & $110(79 \%)$ & $117(84 \%)$ \\
\hline $\mathrm{N}$ & 140 & 140
\end{tabular}

\subsection{Structural model: parameters estimation with the CBSs}

Given that most of the individual choices in both the competitive and risk environments are rationalizable, here we discuss the theoretical framework we adopt to model and estimate the proposed form of a utility function that captures preferences for competition and risk. That is, we put structure on the preferences of the subjects in our sample.

Since we have a rich data set at the individual level with 80 observations per subject (40 for competition and 40 for risk preferences), we are able to estimate a unique utility function for each individual. ${ }^{23}$ Knowing the number of utility functions to estimate, we consequently address the functional form to estimate preferences over competition and risk. One advantage of our design is that the CBS creates a natural context to jointly estimate preferences for competition and risk (Andreoni and Sprenger, 2012). Since this paper is the first attempt to estimate preferences for competition, and also such preferences are formed in an environment where risk is present, we follow the classical framework used to measure risk preferences to estimate both preference parameters jointly (Harrison and Rutström, 2008). In particular, we assume a constant relative risk aversion (CRRA) specification to model the utility of income $(X)$ under each payment-scheme as:

$$
U(X)=\frac{X^{1-\alpha-\delta}}{1-\alpha-\delta}+\theta(x, y)
$$

\footnotetext{
${ }^{23}$ Similar to Andreoni and Sprenger (2012), we assume that individuals make choices that are measured without error, and therefore their utility functions are known. We acknowledge this is a simplifying assumption because someone who exhibits a neutral preference for competition in our setting might show a positive (or negative) preference for competition in a wider range of prices. This can decrease the prediction power of our estimation for prices that are outside the range we used in our experiment. However, we believe that the high variety of prices at the individual level present in our setting helps to minimize this concern.
} 
where $x$ is the payoff under the Individual Rate, $y$ is the payoff under the Tournament Rate, $\alpha$ captures risk preferences in the absence of preferences for competition, $\theta$ captures preferences for competition and $\delta$ captures risk preferences in the presence of preferences for competition (i.e., the effect of being in the competitive environment on risk preferences.). ${ }^{24}$ In our first interpretation, preferences for competition affect the utility of income only through the payoffs, which is represented by $\theta$ and also implies that $\delta=0$. Under this interpretation levels of $\alpha>0$ imply risk aversion, $\alpha=0$ imply risk neutrality, and $\alpha<0$ imply risk loving; when measuring risk preferences in the absence of competition. Similarly, levels of $\theta>0$ imply competition aversion, $\theta=0$ imply competition neutrality, and $\theta<0$ imply competition seeking. The parameter $\theta$ aims to capture the interpretation of preferences for competition commonly used in other experimental settings (Gneezy et al., 2003; Niederle and Vesterlund, 2007; Dariel et al., 2017; Buser et al., 2020). This additive parameter does not influence individuals' risk aversion $\alpha$ and it is conceptualized as the taste or distaste that is generated when the payoffs depend on competition.

In our second interpretation of preferences, we allow for an effect of competition preferences on risk preferences. That is, $\delta$ is no longer equal to 0 and it represents the effect of competition on risk attitudes. A value of $\delta>0$ displays risk aversion, $\delta=0$ displays risk neutrality, and $\delta<0$ displays risk loving; when measuring risk preferences in the presence of competition. Importantly, although in Equation $3.1 \alpha$ and $\delta$ are different parameters, when we obtain the expected values of each choice and report the estimates of our second interpretation, the estimated parameter of $\delta$ accounts already for risk preferences $\alpha$ (that is, we assume additive of the curvature terms between the two environment, i.e., $\hat{\delta}=\delta-\alpha$ ). In that way, we interpret $\delta$ as the additional effect that competition has on risk preferences.

Following the approach of Apesteguia et al. (2019), we discretize the CBS choices into 10 equidistant shares to have binary choice data over two lotteries. As a result, for each of the 80 budget lines per individual ( 40 for the competitive environment and 40 for the non-competitive one) we obtain 10 equidistant points, where each point represents a lottery that offers two prizes: the payoffs in case of winning the tournament (or under the Probabilistic Amount) and the Payoff in case of losing the tournament (or under the Certain Amount). Consequently, we calculate the expected utility of each lottery $n$ and individual $i$ assuming expected utility theory (EUT) for two cases. One with competition:

$$
E U_{n}=\rho_{i} * \frac{x^{1-\delta_{i}}}{1-\delta_{i}}+\left(1-\rho_{i}\right) * \frac{(x+y)^{1-\delta_{i}}}{1-\delta_{i}}+\theta_{i}(x, y)
$$

and the other without competition:

$$
E U_{n}=\beta * \frac{x^{1-\alpha_{i}}}{1-\alpha_{i}}+(1-\beta) * \frac{(x+y)^{1-\alpha_{i}}}{1-\alpha_{i}},
$$

\footnotetext{
${ }^{24}$ Importantly, note that the income $X$ can take two values: if the participant loses the tournament $X=x$ and if she wins the tournament $X=x+y$
} 
where $x$ is the payoff when losing the tournament (or the payoff of the Certain Amount in Equation 3.3),$x+y$ is the payoff when winning the tournament (or the payoff of the Probabilistic Amount in Equation 3.3), $\rho_{i}$ the subjective probability of losing the tournament for individual $i$ under the competitive environment, and $\beta$ is the probability of receiving the Certain amount under the non-competitive environment (which is always equal to 20\%).$^{25}$ As in Harrison and Rutström (2008), the EU for each lottery pair is calculated for a candidate estimate of $\alpha_{i}, \delta_{i}, \theta_{i}$, and the index

$$
\nabla E U=E U_{R}-E U_{L},
$$

is calculated, where $E U_{R}$ is the right lottery and $E U_{L}$ is the left lottery. ${ }^{26}$ The index in Equation 3.4 is obtained with the individual preferences, and thus, is mapped to the observed choices using a standard cumulative normal distribution function $\phi(\nabla E U) .{ }^{27}$ This is a Probit function that transforms any value between $\pm \infty$ into a number ranging between zero and one. ${ }^{28}$ Hence, $\phi(\nabla E U)$ is interpreted as the probability of choosing the right lottery and it is used to define the likelihood values. ${ }^{29}$ Therefore, conditional on the CRRA specification, and EUT being true, an individual's response depends on the estimates of $\alpha_{i}, \delta_{i}$ and $\theta_{i}$, given our statistical specification and the observed choices.

\section{Estimation of preferences for competition and risk}

As a final step, we estimate all three preference parameters $(\alpha, \delta$, and $\theta$ ) jointly following the maximum likelihood specification proposed by Harrison and Rutström (2008). All the estimates use clustering at the individual level to correct the standard errors. Our identification strategy consists of two approaches. In Model 1, we aim to capture our first interpretation of preferences, where competition does not influence risk preferences as implicitly suggested in previous experimental studies (Gneezy et al., 2003; Niederle and Vesterlund, 2007; Dariel et al., 2017; Buser et al., 2020). In Model 2, we illustrate our second interpretation by accounting for the

\footnotetext{
${ }^{25}$ Note that the $E U_{n}$ in Equation 3.2 measures utility cardinally, which makes it impossible to compare the additive term between individuals with differences in the curvature. Hence, we normalize $\theta_{i}$ by the difference between $U_{\max }-U_{\min }$, where $U_{\max }(x)$ is the utility derived for the maximum payoff and $U_{\min }(x)$ is the utility derived for the minimum payoff.

${ }^{26}$ As a result of the discretization of the CBS choices into 10 equidistant binary choice data, the left and risk lotteries correspond to two consecutive points in the budget line (Apesteguia et al., 2019).

${ }^{27}$ Note that the values of $\alpha_{i}$ are identified from the 80 choices made both in the competition and risk preferences elicitation tasks described in Section 3, Part 3 and Part 5. The values of $\delta_{i}$ and $\theta_{i}$ are obtained only from the competition preferences elicitation task described in Section 3, Part 3.

${ }^{28}$ As discussed in Harrison and Rutström (2008), the Logit function leads to a very similar specification.

${ }^{29}$ Given that $\operatorname{Prob}($ choosing right lottery) $=\phi(\nabla E U)$, we assume that the right lottery is chosen when the following holds: $\phi(\nabla E U)>1 / 2$.
} 
possibility of a relation between competitive and risky behavior. This second interpretation seems relevant as recent literature suggests that risk preferences change with the environment (Andersen et al., 2008; Kettlewell, 2019; Schildberg-Hörisch, 2018). Furthermore, ignoring the link between competition and risk can result in an overestimation of preferences for competition (Gillen et al., 2019; van Veldhuizen, 2017).

In formal terms, in Model 1 we impose the restriction of $\delta_{i}=0$, and estimate risk preferences and additive preferences for competition (i.e., $\alpha_{i}$ and $\theta_{i}$ ). In Model 2, we estimate all three parameters together, $\alpha_{i}, \delta_{i}$ and $\theta_{i}$. We conceptualize the parameter $\delta_{i}$ as the effect of competition on risk preferences. Hence, for the estimated parameters displayed in Table 3.3, we report the parameter $\delta_{i}$, after subtracting the parameter $\alpha_{i}$ from it. In that way, $\delta_{i}$ is interpreted as the risk aversion level in a competitive environment (after accounting for the effect that preferences for competition have on it, i.e., $\hat{\delta}=\delta-\alpha)$.

Table 3.3 shows the individual median estimates of preferences for risk and competition, including means, standard deviations and $p$-values of a signed-ranks test for the parameters' median values for Models 1 and $2 .{ }^{30}$ For Model 1, Table 3.3 displays the estimates of risk aversion in the absence of competition $\left(\alpha_{i}\right)$ and competition aversion $\left(\theta_{i}\right)$ jointly. For Model 2, it displays the parameters of risk aversion in the absence of competition $\left(\alpha_{i}\right)$, competition aversion $\left(\theta_{i}\right)$, and risk aversion in the presence of competition $\left(\delta_{i}\right)$ when estimating them jointly. The coefficients for both models suggest that individuals are risk averse in the absence of competition with a median $\alpha_{i}$ of 0.345 and 0.391 , respectively. We also observe that the median value for risk aversion in the absence of competition is significantly greater than zero for both models (Table 3.3, $p<0.01$ and $p<0.01$, for Model 1 and Model 2, respectively).

Table 3.3 also indicates that individuals are competition aversion with a $\theta_{i}$ of -0.616 and -0.897 , for Model 1 and Model 2 respectively, and the median value is significantly smaller than zero for both models (Table 3.3, $p<0.01$ and $p<0.01$, for Model 1 and Model 2, respectively). Interestingly, once we account for the structural interaction between preferences for competition and risk in Model 2, we observe that individuals are slightly risk loving with a median $\delta_{i}$ of -0.124 which is significantly smaller than zero (Table 3.3, $p<0.01$ Model 2). ${ }^{31}$

Figure 3.4 shows the estimated Probabilistic Density Function (PDF) and the normal Gaussian distribution for both risk aversion without competition $\alpha_{i}$ and additive preferences for competition $\theta_{i}$ resulting from Model 1 . The

\footnotetext{
${ }^{30}$ Estimates at population level are shown in the Appendix A2 in Table 3.7 and reveal the same patterns observed from the individual level estimates presented in Table 3.3.

${ }^{31}$ Note that the estimation for both models is done for all participants in which the estimation converged. For Model 1, the estimation did not converge for 5 subjects out of 140 because all of them have 40 corner choices in the competition task. Similarly, for Model 2, the estimation did not converge for 9 subjects out of 140 because all of them have 40 corner choices in the competition task. Nevertheless, in both models the estimation converged for some subjects that had corner choices in all budget sets. In Appendix A2, we show the results for the analysis without the subjects that have corner choices in all 40 decision sets (i.e., 17 subjects out of 140). We do not observe differences in the parameters compared to the ones reported in the main text.
} 
Table 3.3: Estimated risk and competition preferences at the individual level

\begin{tabular}{|c|c|c|c|c|c|c|c|c|}
\hline & \multicolumn{4}{|c|}{ Model 1} & \multicolumn{4}{|c|}{ Model 2} \\
\hline & Median & Mean & sd & $p$-value & Median & Mean & sd & $p$-value \\
\hline$\alpha_{i}$ & $0.345 * * *$ & 0.299 & 0.182 & 0.000 & $0.391 * * *$ & 0.354 & 0.224 & 0.000 \\
\hline$\theta_{i}$ & $-0.616 * * *$ & -1.611 & 4.777 & 0.000 & $-0.897 * * *$ & -1.452 & 2.054 & 0.000 \\
\hline$\delta_{i}$ & & & & & $-0.124 * * *$ & -0.163 & 0.240 & 0.000 \\
\hline & ibjects & & 135 & & 131 & & & \\
\hline & bs. per subj & & 800 & & 800 & & & \\
\hline & Likelihood & & -447.4 & & -447.607 & & & \\
\hline
\end{tabular}

Note: $* p<0.10,{ }^{* *} p<0.05,{ }^{* * *} p<0.01$ resulting from a signed-ranks test for the median values of each preference parameter compared to zero. The above table reports the Maximum-likelihood estimates of the medians, mean, and the standard deviation (i.e., $s d$ ) of the distributions of risk and competitive preferences. For Model 1, we implement the restriction $\delta_{i}=0$, and risk aversion in the absence of competition $\left(\alpha_{i}\right)$ and additive preferences for competition (i.e., competition aversion, $\theta_{i}$ ) are estimated. For Model 2, no restriction is implemented, and risk aversion in the absence of competition $\left(\alpha_{i}\right)$, additive preferences for competition (i.e., competition aversion, $\left.\theta_{i}\right)$, and risk aversion in the presence of competition $\left(\delta_{i}\right)$ are estimated jointly.

individual-level estimates reveal high levels of risk aversion, with the mode of the distribution close to 0.5 , suggesting that a sizable portion of the generated data may show risk aversion levels above 0.5. The data also suggest that individuals are competition averse with a large part of the population displaying a negative additive term for preferences for competition. ${ }^{32}$

Figure 3.5 displays the estimated PDFs and the normal Gaussian distributions of risk aversion without competition $\alpha_{i}$, additive preferences for competition $\theta_{i}$, and risk aversion with competition $\delta_{i}$ resulting from Model 2. We observe a similar pattern to the one in Model 1 for risk aversion without competition and additive preferences for competition. In particular, the majority of the population displays risk aversion levels around 0.5 when there is no competition and an additive level of preferences for competition below zero. In addition, for risk preferences with competition (i.e., $\delta_{i}$ ), we observe that the distribution of individual choices is shifted towards the left in comparison to the one of $\alpha_{i}$, with the mode of the distribution close to -0.25 . This suggests that competition makes individuals to display lower levels or risk aversion. ${ }^{33}$

Summarizing, the results from Table 3.3, Figure 3.4, and Figure 3.5 indicate that most of the participants in our sample display an aversion towards risk when there is no competition in the environment and that

\footnotetext{
${ }^{32}$ Note that for graphical purposes Figure 3.4 shows the estimates values of $\theta_{i}$ ranging between 2 and -6 . This is the case for 131 subjects out of 135 while 4 subjects have estimate values of the additive preferences for competition below -6 .

${ }^{33}$ Note that for graphical purposes Figure 3.5 displays the estimates values of $\theta_{i}$ that range between 2 and -6 . This is the case for 127 subjects out of 131 because 4 subjects have estimate values of the additive preferences for competition below -6 .
} 

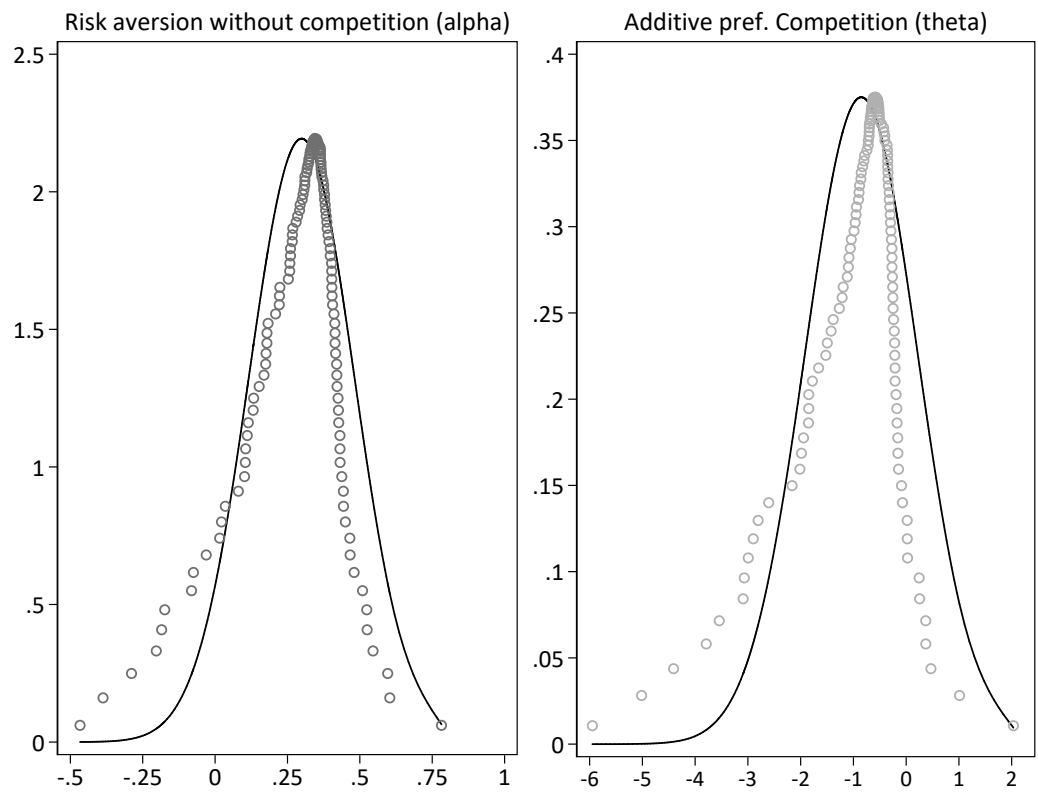

Figure 3.4: PDFs of the estimates reported in Table 3.3 for Model 1. The reference distribution in black is a normal Gaussian.
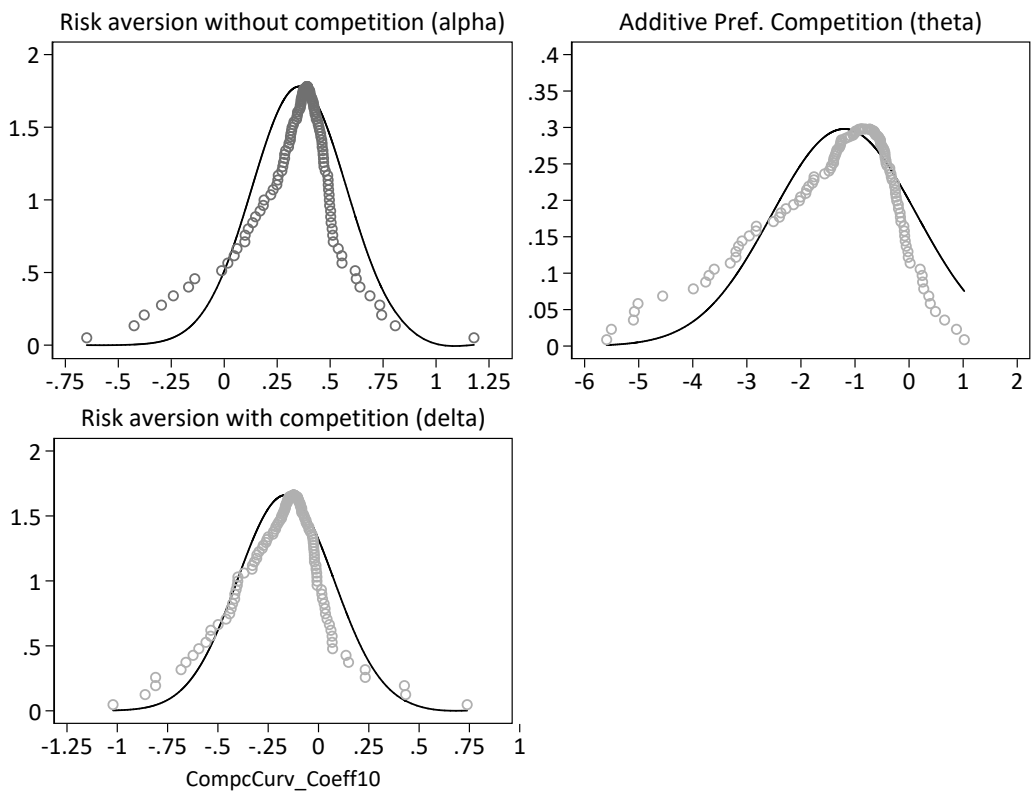

Figure 3.5: PDFs of the estimates reported in Table 3.3 for Model 2. The reference distribution in black is a normal Gaussian.

participants are on average competition averse as the additive preference for competition is negative. Furthermore, we observe that participants become less averse to risk when we account for the effect of preferences for competition on risk attitudes. We provide a further discussion on the implication of this finding in Section 5. 


\subsection{Gender differences}

In this subsection, we test whether there are gender differences in the outcomes presented above, both in terms of consistency and preference estimates.

\section{Testing rationality}

We check first whether there are gender differences in our measurement of rationality by looking at violations of the GARP and CCEI scores in a risky and competitive environment. Figure 3.6 suggests that men and women have a similar distribution in the scores of CCEI in a risk environment. A Kruskal-Wallis non-parametric test confirms this visual impression and shows that there are no significant gender differences for the number of GARP violations $(p=0.188)$ or the CCEI scores $(p=0.125)$. Similarly, we do not observe gender differences in the proportion of men and women with a CCEI above a threshold of 0.90 and 0.95 (Two-sample test of proportions, $p=0.306$ and $p=0.327$, respectively for each threshold).

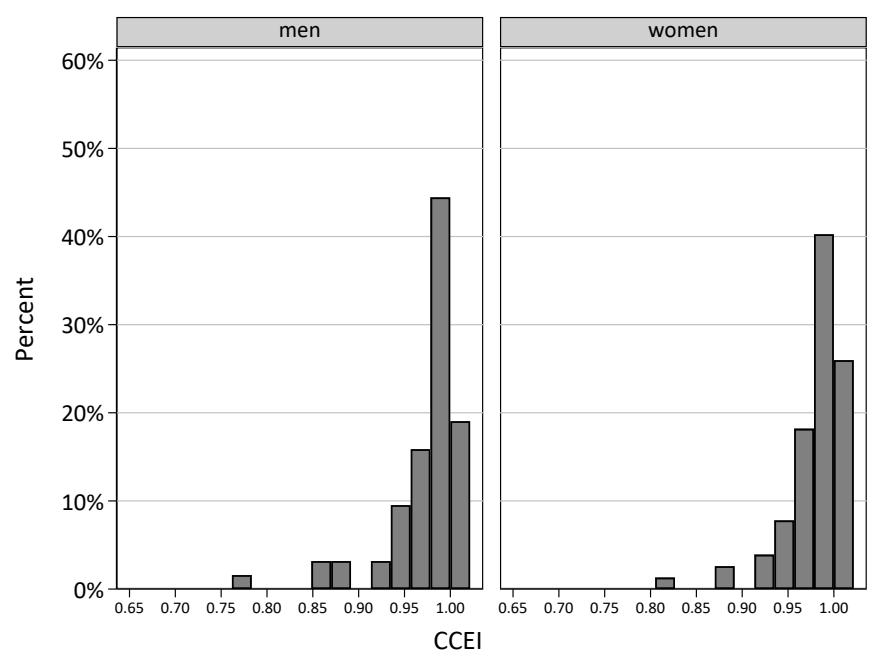

Figure 3.6: CCEI for risk preferences

Figure 3.7 reveals a similar pattern in the consistency for a competitive environment. A Kruskal-Wallis non-parametric test shows that there are no gender differences in the number of GARP violations $(p=0.650)$ or in the CCEI score $(p=.771)$. In addition, there are no statistically significant differences in the proportion of men and women with a CCEI above a threshold of 0.90 and 0.95 (Two-sample test of proportions, $p=0.770$ and $p=0.301$, respectively for each threshold).

We can conclude that there are no gender differences in our consistency measurements and we can thus compare the preference estimates between men and women in the following subsection. 


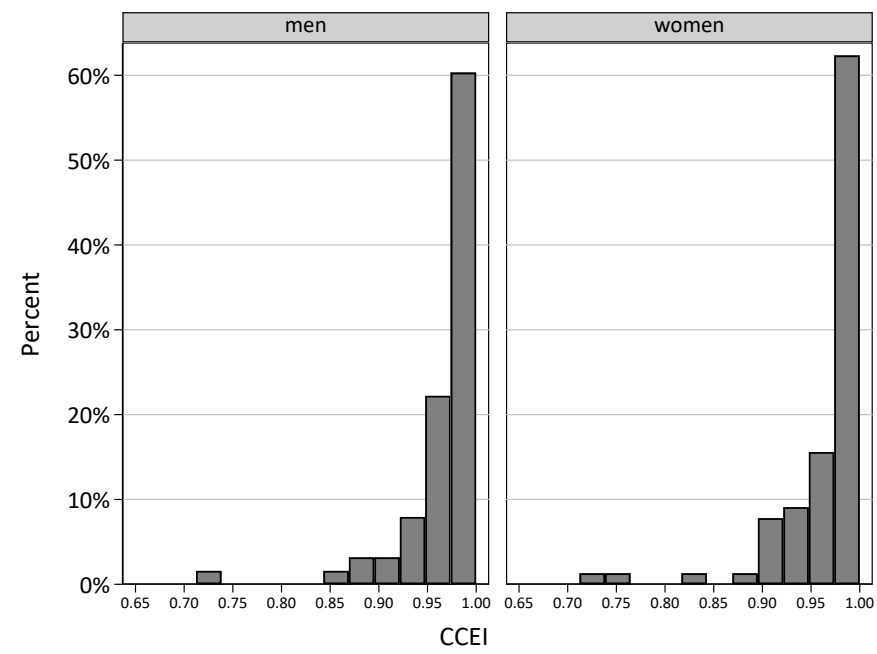

Figure 3.7: CCEI for preferences for competition

\section{Structural model: parameters estimation with the CTB}

Table 3.4 presents the individual median estimates and standard deviations for preferences for risk and competition for Model 1 and Model 2, separated by gender. In general, the coefficients $\alpha_{i}$ and $\theta_{i}$ estimated from both models suggest that women are risk averse in the absence of competition and are competition averse (i.e., $\alpha_{i(\text { Model } 1)}=0.367, \alpha_{i(\text { Model } 2)}=0.399$; $\left.\theta_{i(\text { Model } 1)}=-0.707, \theta_{i(\text { Model } 2)}=-0.996\right)$. Model 2 suggests that women are slightly risk loving seeking in the presence of competition with a $\delta_{i}$ of -0.116 . The coefficients from both of the models suggest that men are also risk averse in absence of competition (i.e., $\alpha_{i(\text { Model } 1)}=0.304$, and $\left.\alpha_{i(\text { Model } 2)}=0.364\right)$, and dislike in general competition (i.e., $\theta_{i(\text { Model } 1)}=-0.428$ and $\left.\theta_{i(\text { Model } 2)}=-0.648\right)$. Lastly, Model 2 suggests that men are risk loving in the presence of competition with with a $\delta_{i}$ of -0.126 .

Consistent with the results provided in Table 3.9 in Model 1, two separate Wilcoxon Rank Sum Mann-Whitney tests suggest that women are more risk averse than men in absence of competition $(p<0.001)$ and are more competition averse when compared to men $(p<0.05)$. Regarding Model 2, two separate Wilcoxon Rank Sum Mann-Whitney tests reveal also that women are more risk averse than men in absence of competition $(p<0.001)$ and display more aversion towards competition than men $(p<0.05)$. Interestingly, when it comes to risk aversion levels in the presence of competition $\delta_{i}$, we do not observe gender differences $(p<0.911) .{ }^{34}$

\footnotetext{
${ }^{34}$ In Appendix A3 we show the estimated PDFs of preferences for both Models by gender. We observe the same patterns as in Table 3.4 and the results of the non-parametric tests discussed in this section.
} 
Table 3.4: Estimated risk and competition preferences by gender

\begin{tabular}{cccccc}
\hline & \multicolumn{2}{c}{ Women } & & \multicolumn{2}{c}{ Men } \\
\cline { 2 - 3 } \cline { 5 - 6 } & Model 1 & Model 2 & & Model 1 & Model 2 \\
\hline$\alpha_{i}$ & $0.367 * * *$ & $0.399 * * *$ & & $0.304 * * *$ & $0.364 * * *$ \\
& {$[0.111]$} & {$[0.124]$} & & {$[0.221]$} & {$[0.293]$} \\
$\theta_{i}$ & & & & & \\
& $-0.707 * * *$ & $-0.996 * * *$ & & $-0.428 * * *$ & $-0.648 * * *$ \\
$\delta_{i}$ & {$[4.380]$} & {$[2.231]$} & & {$[5.261]$} & {$[1.784]$} \\
& & & & \\
& & $-0.116 * * *$ & & $-0.126 * * *$ \\
\# Subjects & & {$[0.177]$} & & & {$[0.305]$} \\
\# Obs. per subjects & 800 & 800 & & 800 & 800 \\
Log-Likehood & -454.274 & -456.903 & & -438.707 & -435.538 \\
\hline
\end{tabular}

Note: ${ }^{*} p<0.10,{ }^{* *} p<0.05,{ }^{* * *} p<0.01$ resulting from a signedranks test for the median values of each preference parameter compared to zero. The above table reports the Maximum-likelihood estimates of the medians, the standard deviation [in parenthesis] of the distributions of risk, and competitive preferences. For Model 1 , we implement the restriction $\delta_{i}=0$, and risk aversion in the absence of competition $\left(\alpha_{i}\right)$ and additive preferences for competition (i.e., competition aversion, $\theta_{i}$ ) are estimated. For Model 2, no restriction is implemented, and risk aversion in the absence of competition $\left(\alpha_{i}\right)$, additive preferences for competition (i.e., competition aversion, $\left.\theta_{i}\right)$, and risk aversion in the presence of competition $\left(\delta_{i}\right)$ are estimated jointly.

\section{Discussion and conclusions}

This study provides the first evidence of consistency of individual choices in a competitive environment and provides structural individual-level estimates of such preferences. We use an experimental design that modifies the experimental task developed by Niederle and Vesterlund (2007), by introducing variation in the stakes at the individual level and implementing the choice between the two payment-schemes as a continuous choice from a CBS. This new feature of our design generates a rich data set at the individual level that allow us first to test whether individual choices in a competitive environment satisfy GARP and can thus be rationalized by a utility function; and second, to propose an economic model to capture such preferences structurally with two different interpretations. The first interpretation follows the classical approach in the literature where preferences for competition impact the utility function of income only through payoffs. The second interpretation allows for an effect of preference for competition on risk preferences. Our results reveal the existence of a highly consistent preference for competition captured by both of our interpretations and suggest a strong link between risk and competition preferences.

In the first part of the result section, we investigate whether individual choices in a competitive environment can be rationalized by a utility function. 
For that purpose, we provide a consistency analysis of competitive behavior by looking at violations of GARP with the help of the CCEI. Our results reveal that $94 \%$ of participants have a CCEI above 0.90. Similarly, we observe high consistency levels of individual choices for risk preferences with also $94 \%$ of participants having a CCEI above 0.90 . These results provide the first evidence of consistency of individual choices in a competitive environment, allowing us to propose a utility function to represent preferences for competition and estimate them structurally. Importantly, we do not find significant differences in violations of GARP between preferences for competition and risk. This is relevant for our joint estimation procedure, since we need to ensure individual choices are consistent in both a risky and competitive environment to estimate simultaneously both preferences.

After the consistency check, we present a joint parametric estimation of preferences for competition and risk at the individual level. Joint estimation of preferences allows considering scenarios where the perception of risk changes due to the competitive environment, as suggested for the case of risk attitudes in other preferences domains (Abdellaoui et al., 2007; Andersen et al., 2008; Kettlewell, 2019; Schildberg-Hörisch, 2018). In addition, it reduces the well-documented curvature bias that results from ignoring structural relation between preferences (Andersen et al., 2008). Using the rich data set generated by the CBS design, we assume a CRRA specification for the utility of income and propose two economic models to represent individual choices in a competitive and risk environment. In the first model, we estimate risk aversion in the absence of competition and additive preferences for competition. The results from this first model suggest that participants are on average risk averse when there is no competition and are competition averse as the additive term is negative. Since this first model ignores a structural relation between preferences for competition and risk (meaning that preferences for competition do not affect risk preferences), we estimate a second model where we allow for relations between both parameters in the curvature of the utility function. In particular, in our second model, we estimate together risk aversion in the absence of competition, additive preferences for competition, and risk aversion in the presence of competition. Similar to the first model, the estimates from the second model suggest that participants are on average risk averse when there is no competition and dislike generally competition captured by the additive term. Interestingly, the results from the second model change the level of risk aversion, that is, when comparing risk attitudes in a competitive versus in a non-competitive environment. Specifically, the curvature term representing risk aversion with competition suggests that participants are on average risk loving.

The finding that risk aversion is malleable with the environment is not new. A large body of literature has studied extensively within variation in risk preferences in settings that track individuals across time (Chuang and Schechter, 2015; Mata et al., 2018). Although some have suggested that such variation is due to noise (Salamanca, 2018), there is evidence of a deterministic source of such variation, for instance, in the case of natural events, health shocks, life events, and many more (for review, see, e.g., 
Kettlewell, 2019). Although we do not track individuals across time, we believe that the differences between the levels of risk aversion experienced in a competitive and in a non-competitive environment are informative to understand better the source of variation of risk preferences within individuals. This seems crucial to understand as systematic changes in risk preferences can have consequences in real-life situations such as labor market and health outcomes, migration decisions, addictive behavior, and investment (for review, see, e.g., Schildberg-Hörisch, 2018).

Our findings also reveal that preferences for competition and risk are related to each other. In fact, relying only on the results from Model 1, where risk and competition are treated as independent terms might result in biased estimation of risk preferences in the population. This is because Model 1 ignores that individuals might display different levels of risk when exposed to a competitive and non-competitive environment. The curvature parameter of preferences for competition present in Model 2 takes this into account, by allowing preferences for competition to affect risk preferences. The finding that individuals are risk loving once we account for individual preferences for competition suggests that their tolerance towards risk scenarios - such as one recreated by a winner-takes-all competition we use in our setup- is influenced by their attitudes towards competition. ${ }^{35}$ This dependency between preferences for competition and risk has been recently suggested by Gillen et al. (2019) and van Veldhuizen (2017), in the context of gender differences in competition. Given the relevance of competition in our daily lives and also the inherent presence of risk in most of the competitive environments, we believe further research is needed to precisely address the role of risk preferences on shaping preferences for competition. ${ }^{36}$

The results from both models also imply that regardless of individual risk attitudes, participants exhibit a general dislike for competition captured by the negative additive term. We interpret this competition aversion as a measure of the intensity of such preferences. Specifically, this additive term influences the utility function of income only through payoffs. We consider that this finding is relevant as it speaks to the importance of accounting for changes in the environment, such as different stakes, in shaping individual preferences for competition. Recent evidence suggested already the relation between stakes and preferences for competition (Petrie and Segal, 2015; Flory et al., 2015; Ifcher and Zarghamee, 2016). However, ours is the first study to account for individual sensitivity of preferences for competition to different stakes, when introducing changes in both the competitive and non-competitive stakes. A venue for future research could be to understand how other changes in the

\footnotetext{
${ }^{35}$ In Appendix A2, Figures 3.9, 3.10 and 3.11 display the scatter plots with the estimated individual mean of risk aversion and preferences for competition for each of participant in the sample. The pattern from both figures suggests a positive relationship between preferences for competition and risk.

${ }^{36}$ In Chapter 2 of this dissertation, we discuss an attempt to estimate preferences for competition irrespective of risk preferences. Specifically, we modify the NV design to control for risk preferences by design and obtain a measure of individual preferences for competition that resembles the additive preference for competition discussed in this chapter.
} 
competitive environment shape individual preferences. For instance, analyzing the effect of other types of competition, not only winner-takes-all, on shaping individual preferences for competition could be an interesting endeavor. We also consider it important to acknowledge that a sizable part of the population has a general dislike for competitive environments when it comes to a direct impact on their payoffs. We argue this can help to inform policies oriented to increase the selection of highly qualified individuals into competitive environments. For instance, one could decrease competition aversion by reducing the losses obtained in case of not winning the tournament to increase participation of competition-averse individuals in tournaments.

One of the advantages of our estimation approach is that it allows us to account for individual heterogeneity in both competition and risk preferences by estimating preferences as a linear function of individual characteristics. Identifying the link between preferences and individual characteristics is relevant as it can help to design policies that target specific demographic groups. ${ }^{37}$ The results from this analysis confirm the well-documented relation observed between risk aversion and gender, as women are more risk averse than men (for review, see, e.g., Meissner et al., 2020). We also identify a relation between age and risk aversion, as older participants are less risk averse than younger participants. Although there is not a consensus about the relation between age and risk preferences, our results are in line with some other previous evidence that confirms this negative relationship between age and risk aversion (Harrison et al., 2007; Andersen et al., 2010; Dohmen et al., 2010; Andersson et al., 2016). ${ }^{38}$ Interestingly, we do not find a relation between age and preferences for competition. Mayr et al. (2012) show a different pattern, with increasing preferences for competition across the life span until an age around of around 50 years old. However, it is hard to compare both studies as the mean age of our participants is around 20, and there is not much variation in terms of age in our sample. Although some literature in psychology suggests that economic students are more competitive in decomposed games (Van Lange et al., 2011), we do not find a relation between studies in the field of economics and taste for competition in our experimental setting. Lastly, our findings reveal that the more brothers participants have, the less they like a competitive environment. There is not much work on the relation between the number of siblings and their gender on preferences for competition. However, some recent evidence suggests that having an older sister is positively associated with women's preferences for competition and negatively associated with men's preferences for competition (Okudaira et al., 2015). We consider that future research could focus on understanding better how individual heterogeneity in a bigger spectrum of characteristics and a more representative sample interacts with preferences for competition. The results from such work could provide great insights for policymakers who aim to design policies in competitive environments that

\footnotetext{
${ }^{37}$ We present the estimation of preferences accounting for individual heterogeneity in Appendix A4

${ }^{38}$ For exceptions, (see, e.g., Meissner et al., 2020).
} 
target better the population.

Our findings also provide some light on the relation between gender and preferences for competition. In particular, we show that women dislike competition more than men. Extensive work in experimental economics has suggested that women are less willing to select into a competitive environment than men (for reviews, see, e.g., Niederle, 2014; Dariel et al., 2017). Interestingly, and as analyzed in detail in the gender result section, gender differences in competitiveness appear only in the additive term of preferences for competition, but not in the curvature term. Hence, this relation suggests that when we account for the structural relation between risk and competition, there is not a gender gap in competitiveness. However, with our experimental design, we cannot claim that the gender gap disappears when accounting for the relation between preferences for competition and risk, as more detailed comparison between a competitive environment with and without risk will be required to make that claim. ${ }^{39}$

We consider that our findings on gender differences are relevant for the recent discussion in the gender literature around how to explain gender differences in selection into competition. This literature suggests that not accounting properly for the confounding factors present in the competitive environment, such as risk preferences and overconfidence, results in an overestimation of the role of preferences for competition in explaining the gender gap in willingness to compete (Gillen et al., 2019; van Veldhuizen, 2017). Our findings suggest a similar pattern, as gender differences in competition are not present after estimating risk and competition jointly. We believe this result can help to inform policies oriented to increase the representation of women in competitive environments. For instance, targeting other traits such as risk attitudes, overconfidence, and other factors not present in this experiment, could be more effective than targeting the trait of competition itself. The fact that men and women do not seem to have different preferences for competition, in general, suggests that other factors could play a more important role in gender differences in competitiveness. This could also explain why policy interventions such as providing information provision are so successful at the moment of reducing the gender gap in competition (Wozniak et al., 2014; Brandts et al., 2015; Balafoutas and Sutter, 2019).

\footnotetext{
${ }^{39}$ In Chapter 2, we propose a methodological approach to create an environment where we can capture preferences for competition irrespective of risk attitudes. Interestingly, similar to our findings with the curvature term of preferences for competitive provided here, our findings in Chapter 2 suggest that there are no gender differences in competition.
} 


\section{Appendix}

\section{A1. Experimental design details}

Table 3.5: Summary of the earnings for each of the parts of the experimental design.

\section{Part 1 - Individual Rate}

Individual Rate $\mathrm{x}$ (\# correct sums)

Part 2 - Tournament Rate

Tournament Rate x (\# correct sums)

Part 3 - Selection of payment-scheme

If participant does not win the tournament:

Individual Rate $\mathrm{x}$ (\# correct sums)

If participant does win the tournament:

(Individual Rate + Tournament Rate) x (\# correct sums)

Part 4 - Beliefs of future performance

If participant guesses the likelihood of being the winner:

$€ 20$

If participant does not guess the likelihood of being the winner:

$€ 0$

Part 5 - Risk preferences elictation

If the outcome a ten-dice throw is different from 1 or 2 :

The chosen Certain Amount

If the outcome of a ten-dice throw is either 1 or 2 :

(The chosen Certain Amount) + (The chosen Probabilistic Amount)

Note: The beliefs were incentivized using the BSR (Karni, 2009). 
Table 3.6: Summary of intercepts and corner choices in Parts 3 and 5 of the experimental design.

Part 3 - Competition Task

\begin{tabular}{lccccc}
\hline & Obs & Mean & Std. Dev. & Min & Max \\
\hline x intercept & 5,600 & 1193.608 & 580.900 & 108 & 3725 \\
y intercept & 5,600 & 6590.499 & 3095.262 & 626 & 19900 \\
\# corner choices & 5,600 & 11.557 & 14.929 & 0 & 40 \\
\hline \multicolumn{5}{c}{ Part 5 - Risk Task } \\
\hline X intercept & Obs & Mean & Std. Dev. & Min & Max \\
y intercept & 5,600 & 1196.165 & 587.788 & 102 & 3675 \\
\# corner choices & 5,600 & 9572.185 & 3051.579 & 616 & 19950 \\
\hline
\end{tabular}

Note: the 5600 observations are the result of 40 choices for each of the 140 participants. Also, for the risk task, the $\mathrm{x}$ and $\mathrm{y}$ intercepts are adjusted by the number of sums to be comparable to the competition task.

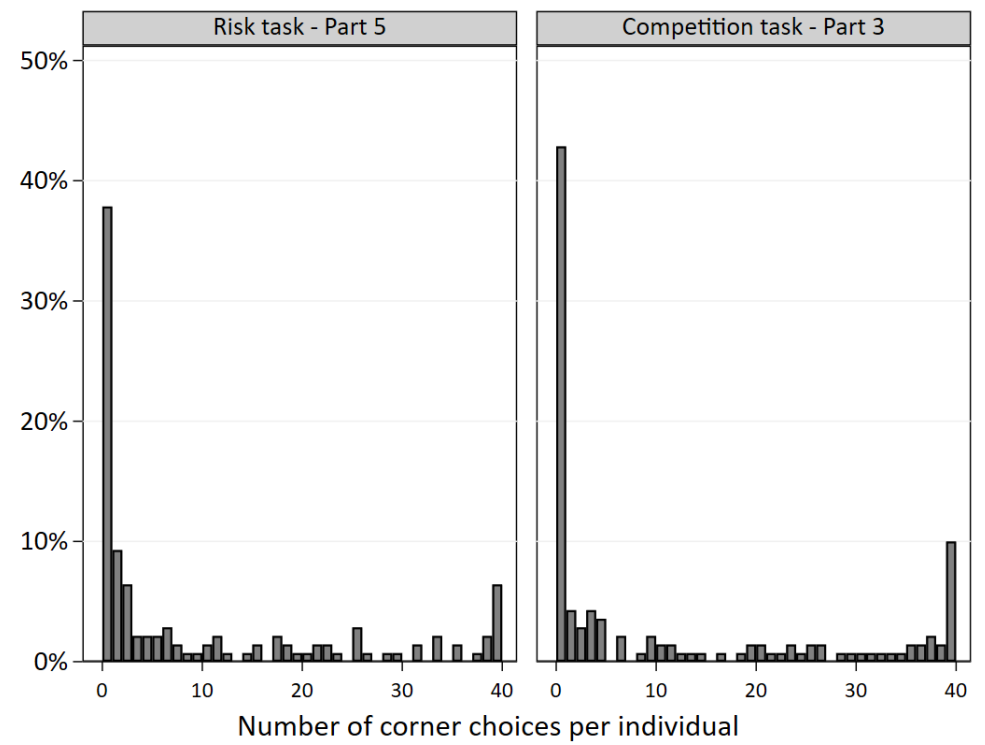

Figure 3.8: Percentage of participants with an specific number of corner choices in the risk and competition tasks. 


\section{A2. Structural model additional features and results}

Table 3.7 contains the resulting point estimates of the preference parameters for the whole sample.

Table 3.7: Estimated risk and competition preferences at Population level

\begin{tabular}{lccccc}
\hline & Parameter & Point estimate & Std. Error & $\begin{array}{c}\text { Lower Bound } \\
95 \% \text { CI }\end{array}$ & $\begin{array}{c}\text { Upper Bound } \\
95 \% \text { CI }\end{array}$ \\
\hline \multicolumn{1}{c}{ Model 1 } & $\alpha$ & 0.295 & 0.025 & 0.247 & 0.344 \\
& $\theta$ & -0.538 & 0.037 & -0.612 & -0.465 \\
\hline \# Obs. & 112,000 & & & & \\
Log-Likelihood & -70387.777 & & & & \\
\hline Model 2 & $\alpha$ & 0.402 & 0.0214 & 0.360 & 0.444 \\
& $\theta$ & -0.900 & 0.136 & -1.168 & -0.633 \\
& $\delta$ & -0.144 & 0.036 & -0.215 & -0.074 \\
\hline \# Obs. & 112,000 & & & & \\
Log-Likelihood & -70190.523 & & & & \\
\hline
\end{tabular}

Note: The above table reports the Maximum-likelihood estimates of the means (medians) and the standard deviation [in parentheses] of the distributions of risk and competitive preferences. The second column shows the results for Model 1 , where the restriction $\delta_{i}=0$ is implemented and risk preferences $\left(\alpha_{i}\right)$ and additive preferences for competition $\left(\theta_{i}\right)$ are estimated. The third column shows the results for Model 2, where no restriction is implemented, and risk preferences $\left(\alpha_{i}\right)$, additive preferences for competition $\left(\theta_{i}\right)$ and curvature preferences for competition $\left(\delta_{i}\right)$ are estimated. 


\section{Model 1}

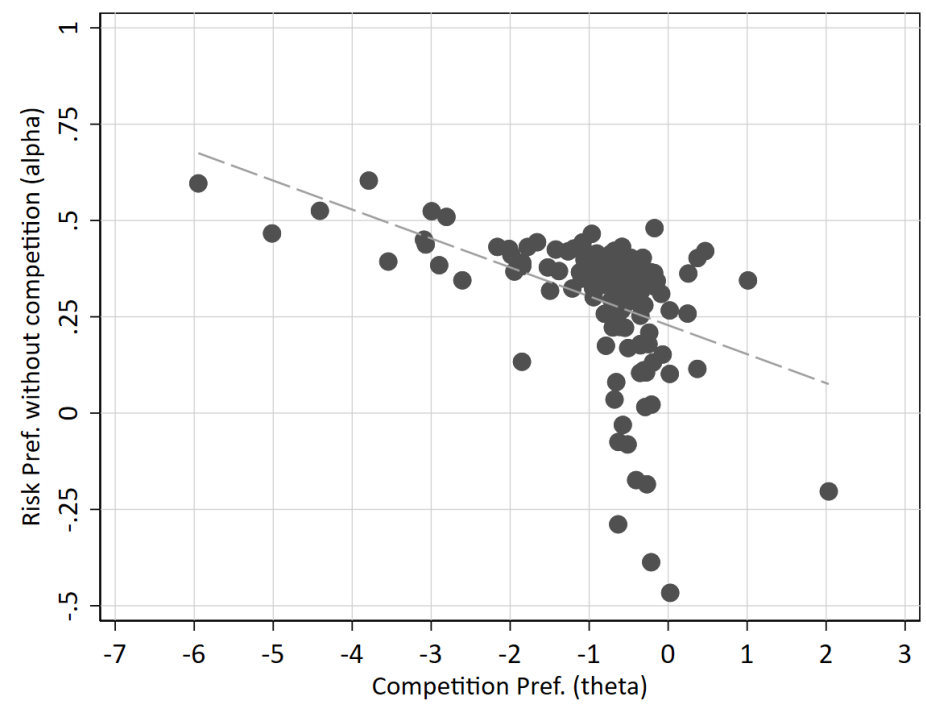

Figure 3.9: Each point represents the estimates of the coefficient of risk aversion $(\alpha)$ and the coefficient for the additive preference for competition $(\theta)$ at the individual level, following the estimation procedure reported in Table 3.3 for Model 1.

\section{Model 2}

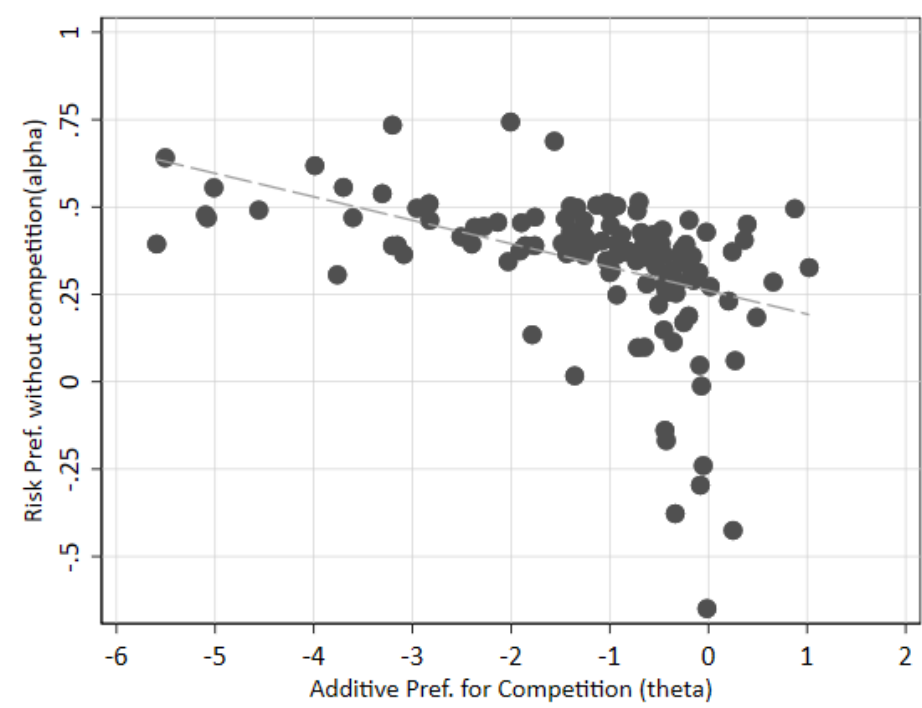

Figure 3.10: Each point represents the estimates of the coefficient of risk aversion without competition $(\alpha)$ and the coefficient for the additive preference for competition $(\theta)$ at the individual level, following the estimation procedure reported in Table 3.3 for Model 2. 


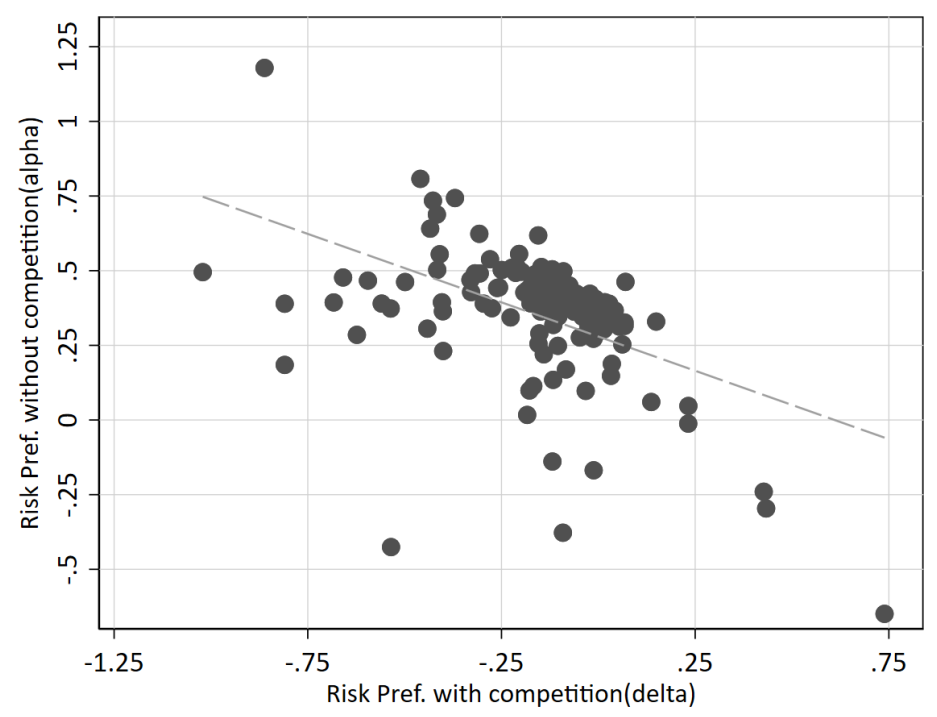

Figure 3.11: Each point represents the estimates of the coefficient of risk aversion without competition $(\alpha)$ and risk aversion with competition $(\delta)$ at the individual level, following the estimation procedure reported in Table 3.3 for Model 2.

\section{Analysis without corner choices}

In this section, we perform the estimation of Models 1 and 2 after removing the individuals that made only corner choices in either the competitive or risk task. That is, we remove 17 participants out 140 .

Table 3.8: Estimated risk and competition preferences at the individual level

\begin{tabular}{|c|c|c|c|c|c|c|}
\hline \multirow[b]{2}{*}{ Parameter } & \multicolumn{3}{|c|}{ Model 1} & \multicolumn{3}{|c|}{ Model 2} \\
\hline & Median & Mean & $\mathrm{sd}$ & Median & Mean & $\mathrm{sd}$ \\
\hline$\alpha_{i}$ & $0.344 * * *$ & 0.316 & 0.152 & $0.392 * * *$ & 0.372 & 0.191 \\
\hline$\theta_{i}$ & $-0.586 * * *$ & -0.998 & 1.634 & $-0.896 * * *$ & -1.488 & 2.081 \\
\hline$\delta_{i}$ & & & & $-0.122 * * *$ & -0.161 & 0.217 \\
\hline \# Subjects & 123 & & & 123 & & \\
\hline \# Obs. per subject & 800 & & & 800 & & \\
\hline Log-Likelihood & -459.882 & & & -455.324 & & \\
\hline
\end{tabular}

Note: $* p<0.10, * * p<0.05, * * * p<0.01$ for a signed-ranks test for the median values of the preference parameters. The above table reports the Maximum-likelihood estimates of the medians, means and the standard deviation (sd) of the distributions of risk and competitive preferences. Columns 2, 3 and 4 show the results for Model 1, where the restriction $\delta_{i}=0$ is implemented and risk aversion $\left(\alpha_{i}\right)$ and additive preferences for competition $\left(\theta_{i}\right)$ are estimated. Columns 5, 6 and 7 show the results for Model 2, where no restriction is implemented, and risk aversion $\left(\alpha_{i}\right)$, additive preferences for competition $\left(\theta_{i}\right)$ and competition aversion $\left(\delta_{i}\right)$ are estimated. 

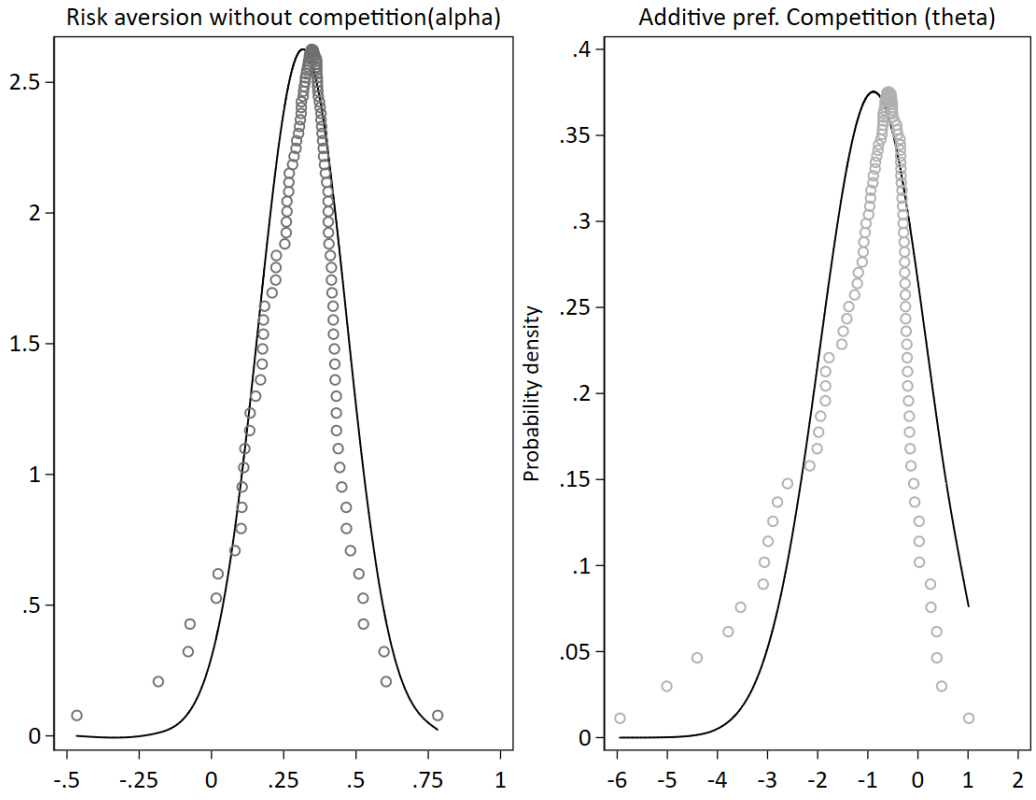

Figure 3.12: PDFs of the estimates reported in Table 3.3 for Model 1. The reference distribution in black is a normal Gaussian.
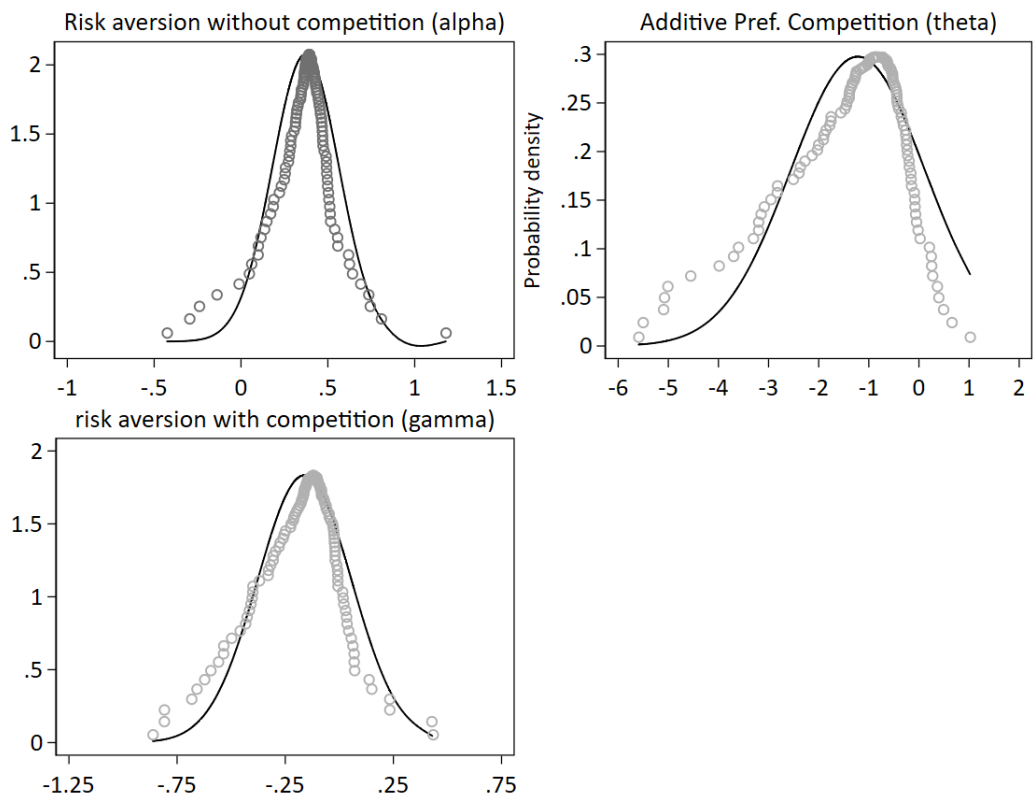

Figure 3.13: PDFs of the estimates reported in Table 3.3 for Model 2. The reference distribution in black is a normal Gaussian. 


\section{A3. Structural estimation by gender}

In this section, we present the figures that complement the analysis presented in Section 4.4 for our gender analysis.
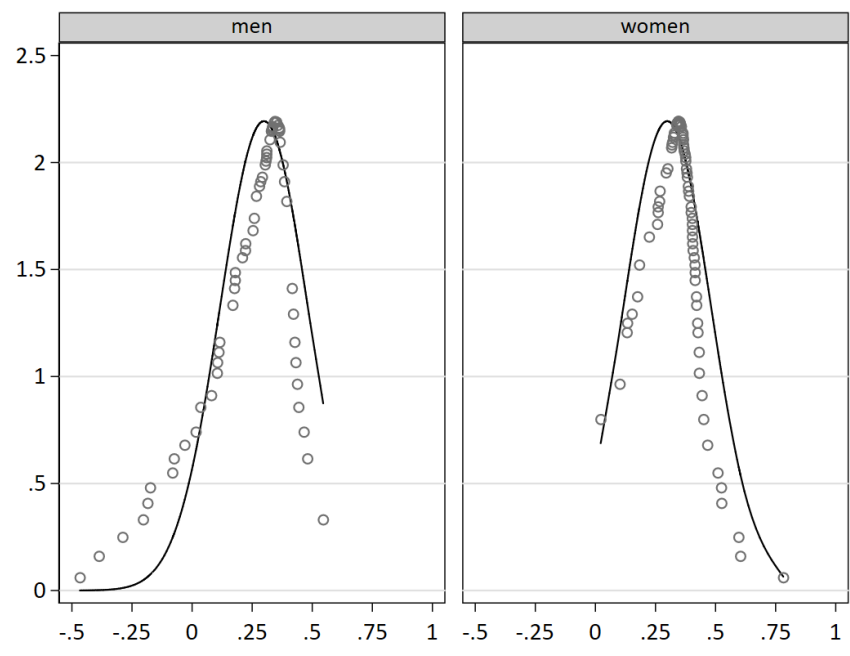

Figure 3.14: PDFs of the estimates reported in Table 3.4 for Model 1 for Risk preferences without competition $(\alpha)$. The reference distribution in black is a normal Gaussian.
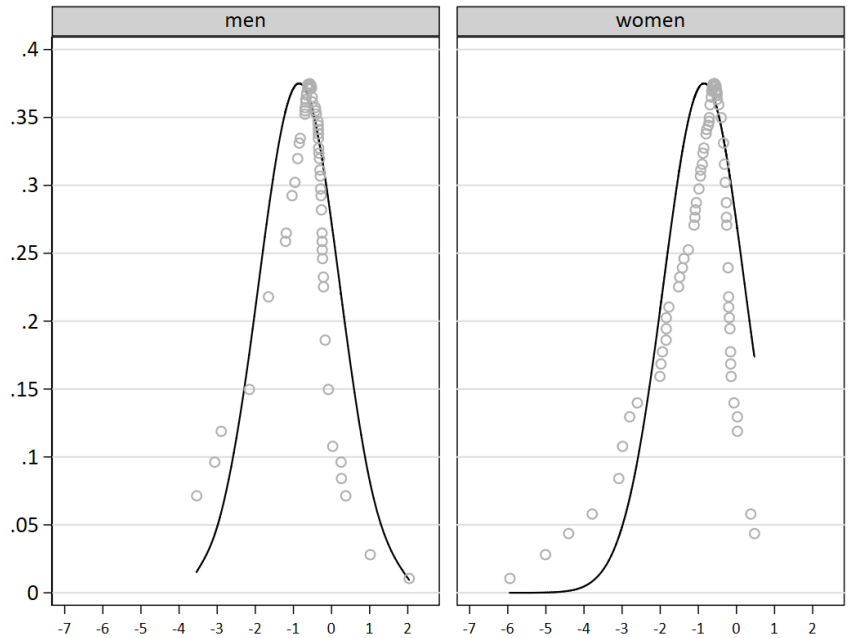

Figure 3.15: PDFs of the estimates reported in Table 3.4 for Model 1 for Competitive preferences $(\theta)$. The reference distribution in black is a normal Gaussian. 


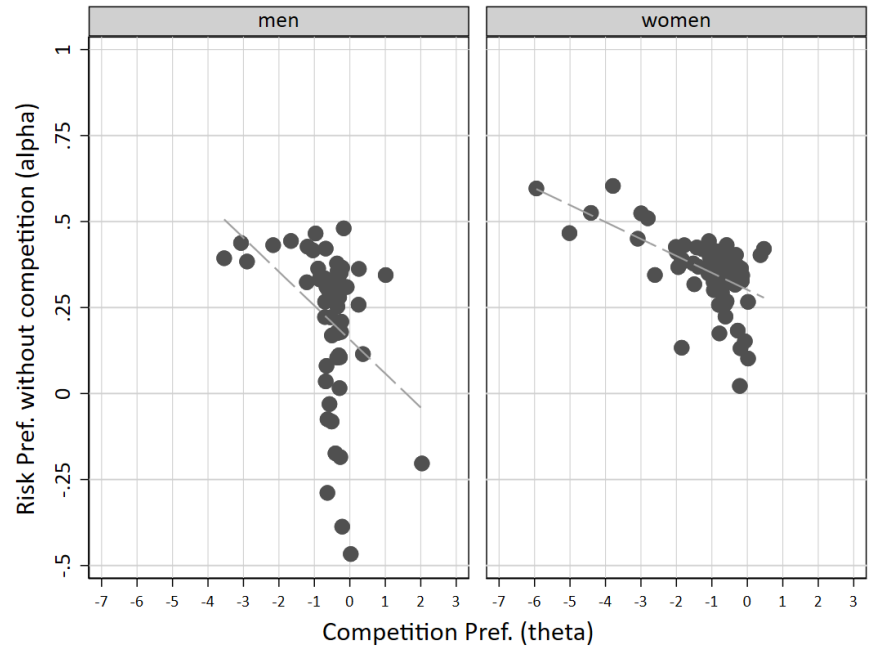

Figure 3.16: Each point represents the estimates of the coefficient of risk aversion without competition $(\alpha)$ and the coefficient for the additive preference for competition $(\theta)$ at the individual level, following the estimation procedure reported in Table 3.4

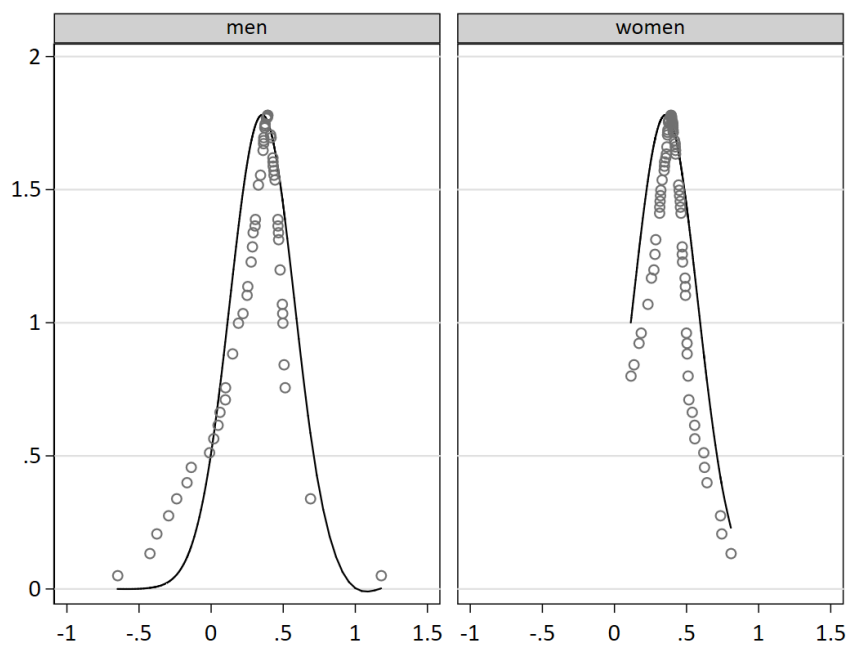

Figure 3.17: PDFs of the estimates reported in Table 3.4 for Model 2 for risk preferences without competition $(\alpha)$. The reference distribution in black is a normal Gaussian. 


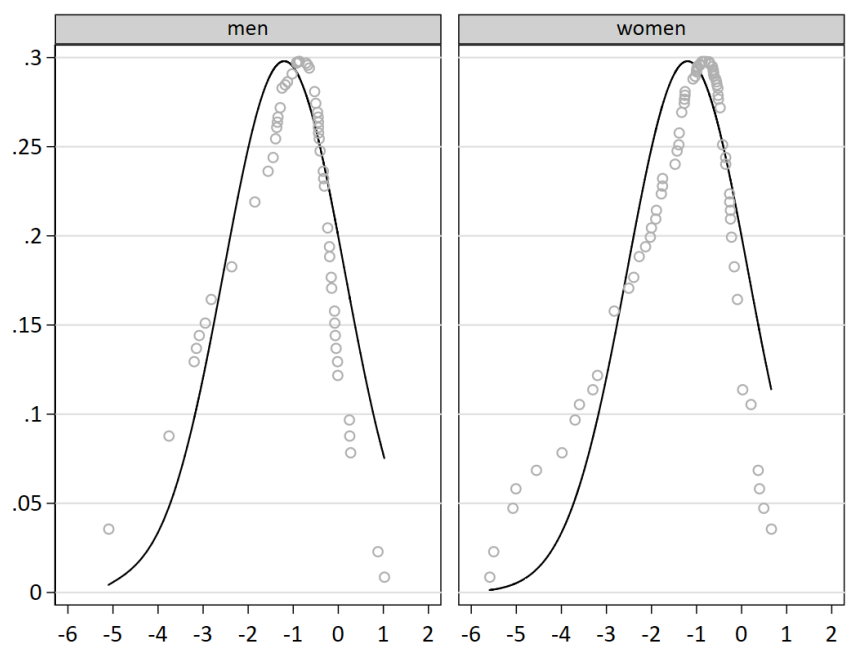

Figure 3.18: PDFs of the estimates reported in Table 3.4 for Model 2 for additive preferences for competition $(\theta)$. The reference distribution in black is a normal Gaussian.

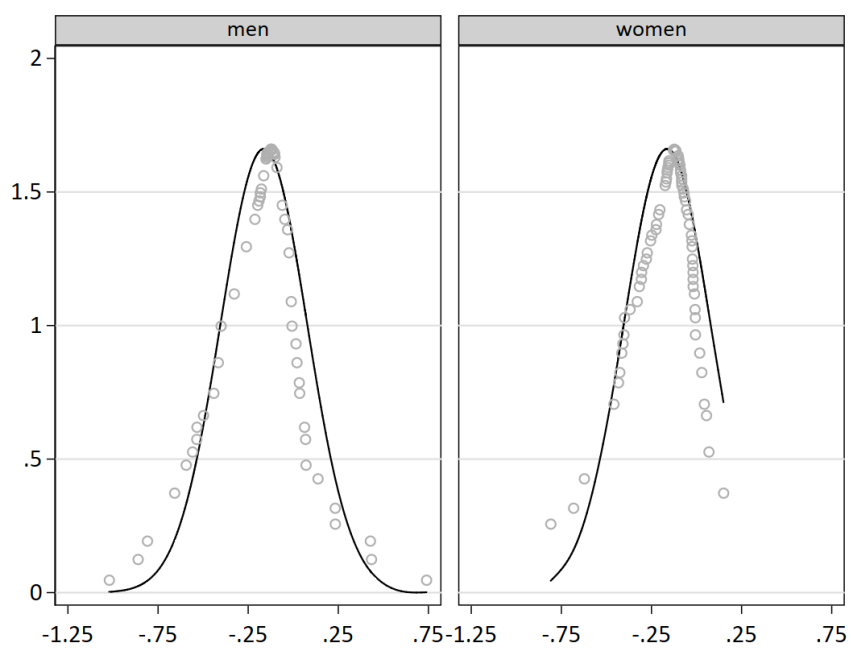

Figure 3.19: PDFs of the estimates reported in Table 3.4 for Model 2 for risk aversion with competition $(\delta)$. The reference distribution in black is a normal Gaussian. 


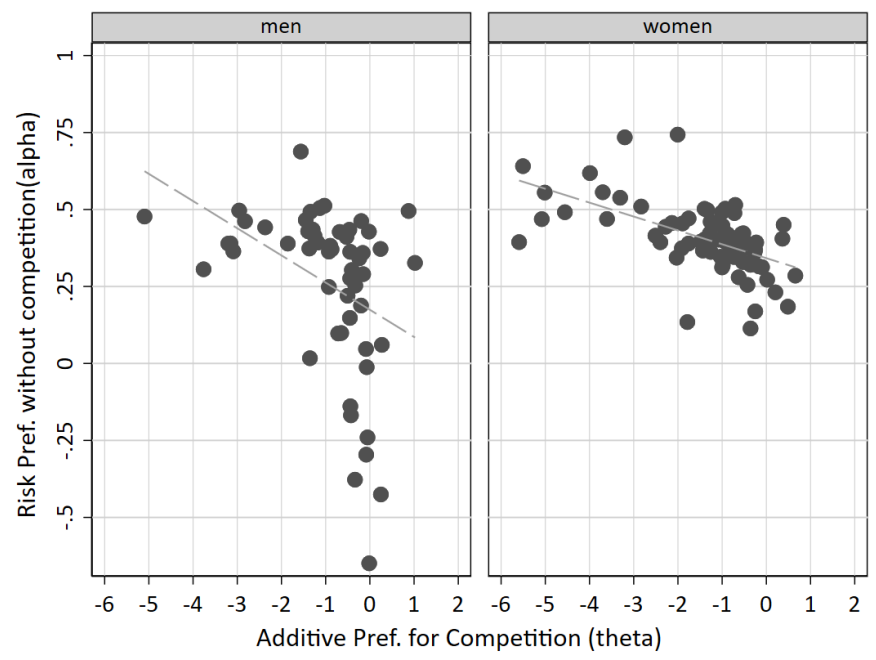

Figure 3.20: Each point represents the estimates of the coefficient of risk aversion without competition $(\alpha)$ and the coefficient for the additive preference for competition $(\theta)$ at the individual level, following the estimation procedure reported in Table 3.4 for Model 2.

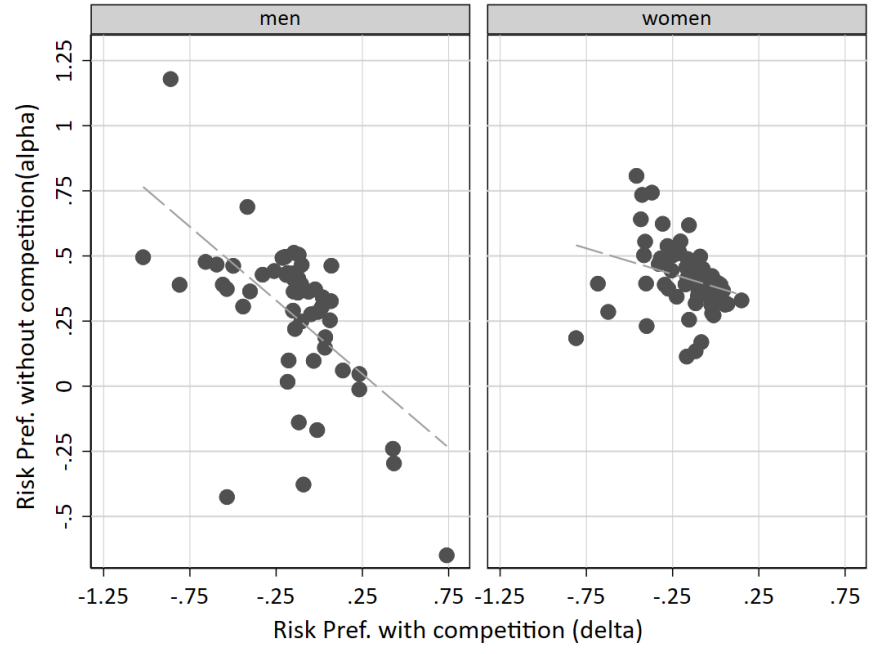

Figure 3.21: Each point represents the estimates of the coefficient of risk aversion without competition $(\alpha)$ and the coefficient for risk aversion with competition $(\delta)$ at the individual level, following the estimation procedure reported in Table 3.4 for Model 2 . 


\section{A4. Individual characteristics and preferences}

In this subsection, we test for the role of individual heterogeneity in shaping individual preferences. In Table 3.9 we test how the preferences parameters in Models 1 and 2 (i.e., $\alpha_{i}, \theta_{i}$ and $\delta_{i}$ ) are related to individual characteristics. In particular, we use logistic models, where the preference parameters are estimated jointly as a linear function of the demographic characteristics of individuals in our sample. We include a dummy variable for Gender that takes the value of one for women; and a dummy variable for Economics that takes the value of one if the field of studies is either economics, business or finance and zero otherwise; a dummy variable for Nationality $E U$ that takes the value of one if the nationality is from an EU country and zero otherwise. We also account for Age; and lastly the number of sisters and brothers individuals grow up with in their household with the variables Sisters and Brothers. ${ }^{40}$

Table 3.9: Relationships between individual characteristics and risk aversion $\alpha_{i}$, additive preferences for competition $\theta_{i}$ and competition aversion $\delta_{i}$.

\begin{tabular}{|c|c|c|c|c|c|}
\hline & \multicolumn{2}{|c|}{ Model 1} & \multicolumn{3}{|c|}{ Model 2} \\
\hline & $\alpha$ & $\theta$ & $\alpha$ & $\theta$ & $\delta$ \\
\hline \multirow[t]{2}{*}{ Gender } & $0.231^{* * *}$ & $-0.979 * * *$ & $0.157 * *$ & $-0.886^{* * * *}$ & 0.054 \\
\hline & (3.31) & $(-4.08)$ & (3.05) & $(-4.14)$ & (1.09) \\
\hline \multirow[t]{2}{*}{ Age } & $-0.012 *$ & 0.024 & $-0.025 * *$ & 0.041 & -0.009 \\
\hline & $(-1.99)$ & (1.76) & $(-2.74)$ & (1.77) & $(-1.08)$ \\
\hline \multirow[t]{2}{*}{ Economics } & -0.043 & 0.216 & -0.132 & 0.881 & 0.044 \\
\hline & $(-1.01)$ & (1.44) & $(-1.53)$ & (1.68) & $(0.93)$ \\
\hline \multirow[t]{2}{*}{ Nationality EU } & -0.016 & -0.059 & -0.208 & 0.021 & -0.191 \\
\hline & $(-0.31)$ & $(-0.21)$ & $(-1.80)$ & $(0.02)$ & $(-1.51)$ \\
\hline \multirow[t]{2}{*}{ \# Sisters } & -0.015 & -0.026 & -0.035 & -0.054 & -0.023 \\
\hline & $(-0.77)$ & $(-0.45)$ & $(-1.34)$ & $(-0.68)$ & $(-0.77)$ \\
\hline \multirow[t]{2}{*}{ \# Brothers } & 0.050 & $-0.197 * *$ & 0.015 & $-0.257^{*}$ & -0.021 \\
\hline & (1.73) & $(-2.68)$ & $(0.46)$ & $(-2.41)$ & $(-0.69)$ \\
\hline \multirow[t]{2}{*}{ Constant } & $0.267 * * *$ & -0.385 & $0.662 * * *$ & -2.148 & -0.280 \\
\hline & $(9.65)$ & $(-1.42)$ & $(3.91)$ & $(-1.48)$ & $(2.44)$ \\
\hline \# Obs. & 112000 & & \# Obs. & 112000 & \\
\hline Log-Likelihood & -69136.903 & & Log-Likelihood & -68689.782 & \\
\hline
\end{tabular}

Standard errors (clustered at the individual level) are shown in parentheses. ${ }^{*} p<0.10, * * p<0.05, * * * p<0.01$

The results from Table 3.9 show a relation between gender and risk aversion in both models, indicating that women are more risk averse than men. We also observe a relation between age and risk aversion in both models, as older individuals are less risk averse. For the additive preferences for competition there is an association between gender and these preferences in both models, as women dislike more competition than men and this difference is significant. Both models also show a relation between the number of brothers and the additive preferences for competition, with a higher number of brothers decreasing the taste for competition. Lastly, the results from Model

\footnotetext{
${ }^{40}$ Please note that we standardize Age by removing the mean population value to each individual's age.
} 
2 indicate that there is no association between competition aversion and the individual characteristics included in Table 3.9. 


\section{A5. Instructions}

[Page 1]

\section{General Instructions}

Welcome to the experiment. ${ }^{41}$ In the experiment today, you will be asked to complete five tasks. Your total earnings at the end of the experiment are the sum of the following two components: (1) A $€ 10$ show up fee; and (2) your earnings in one of the five tasks. Specifically, at the end of the experiment, one of these five tasks will be randomly chosen for payment purposes. Before each task, we will describe in detail how your earnings for that task are determined. In this experiment, we use the currency "Token". The number of tokens you earned will be transformed into Euros at the exchange rate 100 tokens $=€ 1,00$. Once you complete all five tasks, we will call you one by one and pay your earnings in private.

During the experiment, the use of cell phones is prohibited. As it is written down in the consent form you have in the screen, all your information, decisions, and performance during this experiment are anonymous. Please click the "Accept" button to continue with the experiment.

You will receive the instructions for each of the tasks right before you start each task. Now you will start Task 1, please read the instructions of Task 1 carefully.

Welcome to the experiment. In the experiment today, you will be asked to complete five tasks. Your total earnings at the end of the experiment are the sum of the following two components: (1) A $€ 10$ show up fee; and (2) your earnings in one of the five tasks. Specifically, at the end of the experiment, one of these five tasks will be randomly chosen for payment purposes. Before each task, we will describe in detail how your earnings for that task are determined. In this experiment, we use the currency "Token". The number of tokens you earned will be transformed into Euros at the exchange rate 100 tokens $=€ 1,00$. Once you complete all five tasks, we will call you one by one and pay your earnings in private.

During the experiment, the use of cell phones is prohibited. As it is written down in the consent form you have in the screen, all your information, decisions, and performance during this experiment are anonymous. Please click the "Accept" button to continue with the experiment.

You will receive the instructions for each of the tasks right before you start each task. Now you will start Task 1, please read the instructions of Task 1 carefully.

\footnotetext{
${ }^{41}$ The text font, the size and the appearance of images have been adapted from the original instruction version.
} 


\section{[Page 2]}

\section{TASK 1}

In Task 1 you will be given four minutes to calculate a series of sums of four two-digit numbers (see "Screenshot Summation Task"). You cannot use a calculator, but you are welcome to use the provided scratch paper. You submit an answer by clicking the button "Submit". When you submit an answer, the computer will immediately tell you whether the answer is correct or incorrect and a new sum is generated.

Your earnings for Task 1 depend on your individual performance. You will receive 100 Tokens per correctly answered sum. We refer to this payment scheme as individual rate.

Practice round: Before Task 1 starts, you will have two minutes to get familiar with the screen and to practice the calculation of series of sums of four two-digit numbers. Please notice that your answers in this practice round will not be considered for your earnings in this experiment.

Once you are done reading, click on the "NEXT" button on your screen.

Task 1

Sum 1: $\quad 63+34+98+96$

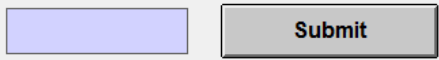

Your last answer was:

Number of correct answers:

0

Seconds left: 8

Figure 3.22: Summation Task 


\section{[Page 3]}

\section{TASK 2}

As in the previous task, you will be given four minutes to calculate sums of four two-digit numbers.

The difference with Task 1 is that, in Task 2, your earnings depend on your performance and the performance of four other participants. Specifically, you will be randomly assigned to a group of five participants. The individual who correctly solves the highest number of sums in the group will be the group's winner. If there are ties the winner will be determined randomly among the tied group members. The earnings for Task 2 are calculated as follows: the group's winner receives 550 Tokens per correctly answered sum while everyone else in the group receives 0 Tokens. We refer to this payment scheme as tournament rate. You will not be informed of how you did in the tournament until you have completed all five tasks.

Once you are done reading, click on the "Next" button on your screen. 


\section{[Page 4]}

\section{TASK 3}

As in the previous tasks you will be given four minutes to calculate sums of four two-digit numbers.

Before performing the task, you choose how you want to be paid for each correct sum in Task 3. You choose a combination of individual rate and tournament rate. To make your choice, you pick a point on a green line in a graph like the one below. The individual rate corresponds to the horizontal axis, and the tournament rate corresponds to the vertical axis. Every point on the green line corresponds to a combination of individual rate and tournament rate. You can only choose one point on the green line, but you are free to choose any point.

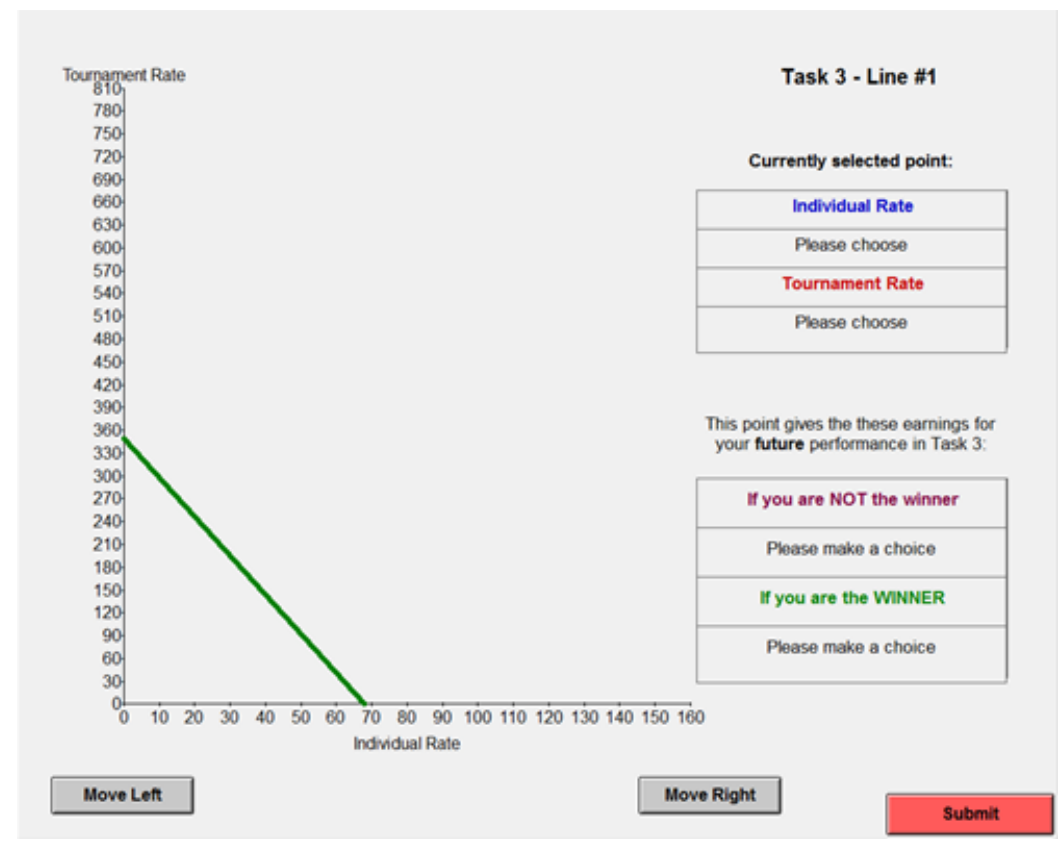

Figure 3.23: Example Task 3

The two payments schemes are as follows:

- A. For each correct sum in Task 3, the individual rate pays the rate corresponding to the point you choose on the green line. For example, if you choose an individual rate of 50 then you will receive 50 Tokens per correct sum.

- B. For each correct sum in Task 3, the tournament rate pays the rate corresponding to the point you choose on the green line if you are the winner of your group in Task 3. Specifically, your performance in Task 3 will be compared with the performance of the four other members of your group in Task 2. You will be your group's winner if you solved more sums in Task 3 than all the other group members in Task 2. If there are ties the winner will be randomly determined among the tied group members. 
If you are not your group's winner, then you will be paid 0 Tokens. For example, if you choose a tournament rate of 350 then you will receive 350 Tokens per correct sum if you are the group's winner and you will receive 0 Tokens if you are not.

In summary, you will be paid in the following way in Task 3:

- - If you are not the group's winner in Task 3:

(Your chosen individual rate) $\times$ (Number of correct sums in Task 3 )

- - If you are the group's winner in Task 3:

(Your chosen individual rate) $\times$ (Number of correct sums in Task 3$)+$ (Your chosen tournament rate) $\times$ (Number of correct sums in Task 3 )

\section{Examples:}

Example 1: Imagine you chose point $A$ in the graph below. In this point, you have an individual rate of 50 Tokens per correct sum and a tournament rate of 92 Tokens per correct sum. This means that:

- - If you are not the group's winner in Task 3, you earn 50 Tokens per correct sum: 50 Tokens from your individual rate choice and 0 Tokens from your tournament rate choice.

- - If you are the group's winner in Task 3, you earn 142 Tokens per correct sum: 50 Tokens from your individual rate choice and 92 Tokens from your tournament rate choice

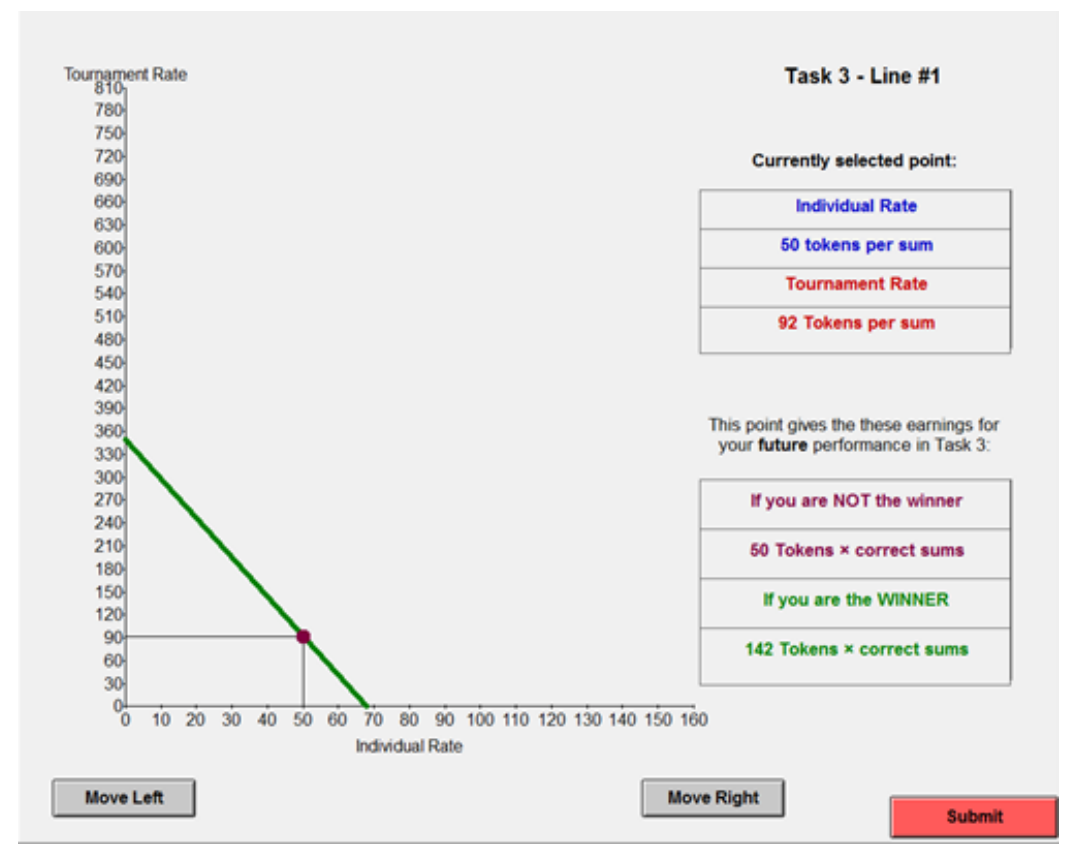

Figure 3.24: Example Task 3

Example 2: Another possible choice is B. In this point, you have an individual rate of 16 Tokens and a tournament rate of 570 Tokens. This means that: 
- If you are not the group's winner in Task 3, you earn 15 Tokens per correct sum: 15 Tokens from your individual rate choice and 0 Tokens from your tournament rate choice.

- If you are the group's winner in Task 3, you earn 585 Tokens per correct sum: 15 Tokens from your individual rate choice and 570 Tokens from your tournament rate choice

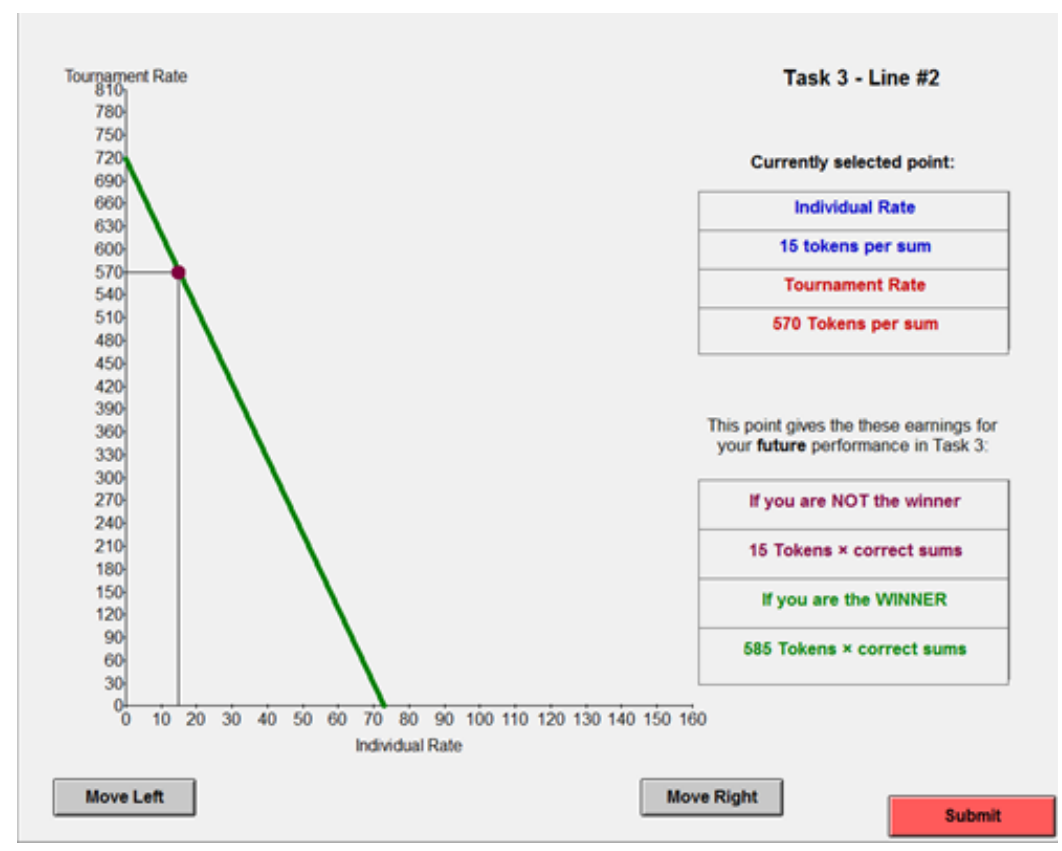

Figure 3.25: Example Task 3

Example 3: Another possible choice is C. In this point, you have an individual rate of 146 Tokens and a tournament rate of 0 Tokens. This means that:

- - If you are not the group's winner in Task 3, you earn 146 Tokens per correct sum: 146 Tokens from your individual rate choice and 0 Tokens from your tournament rate choice.

- - If you are the group's winner in Task 3, you earn 146 Tokens per correct sum: 146 Tokens from your individual rate choice and 0 Tokens from your tournament rate choice 


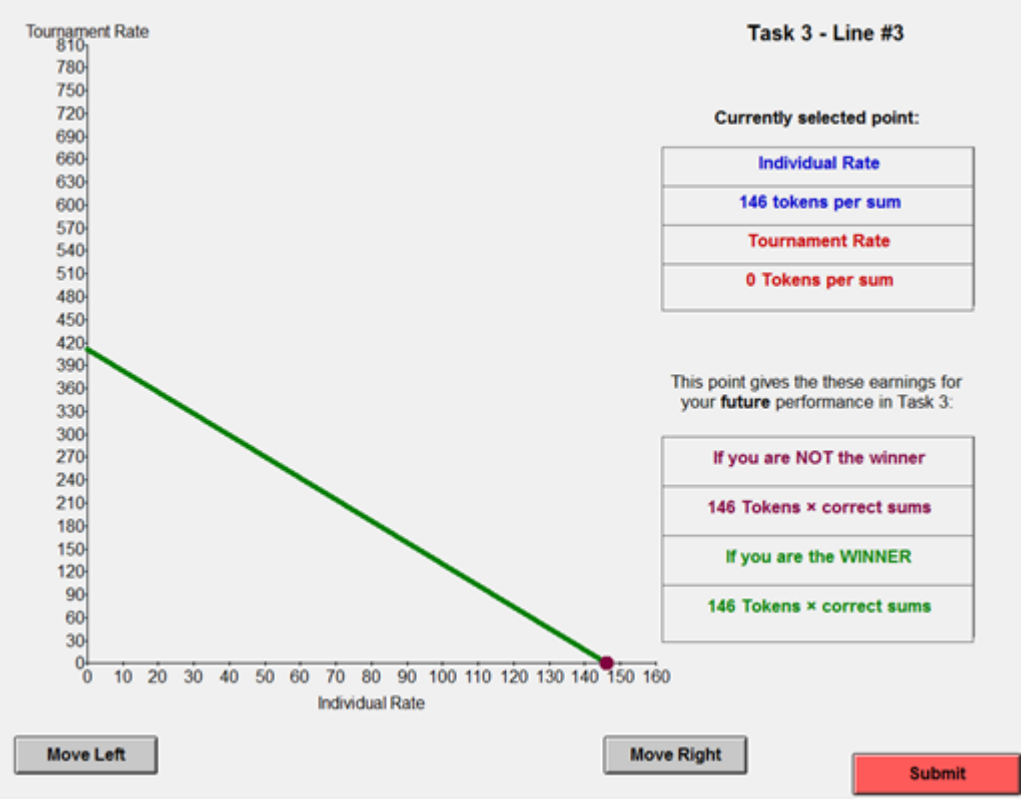

Figure 3.26: Example Task 3 
You will be asked to make a choice in 40 different lines. The green line representing the combinations of individual rate and tournament rate will change from choice to choice. Examples of lines that you might see are shown below. For each choice, the computer randomly selects a green line that crosses the horizontal axis between 50 and 150 Tokens and the vertical axis between 300 and 800 Tokens. After you have made a choice for all 40 lines, one of them will be randomly selected to determine your earnings for Task 3 (each line with equal probability). You will perform the sums for Task 3 knowing the piece-rate and tournament rate that determine your Task 3 earnings.
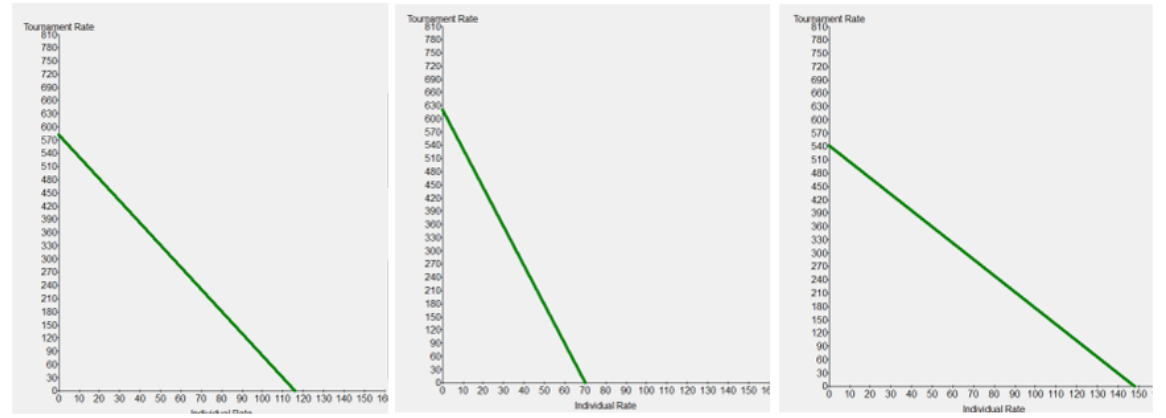

Figure 3.27: Example Task 3

To make a choice, use your mouse to click on a point on the line. Once you click, you will see on the horizontal axis the value for individual rate and on the vertical axis that value for tournament rate. On the right side of the screen, you will also see two tables. The table on the top will display the point you selected on the green line. The table on the bottom will display your earnings (in Tokens) in Task 3 per correct sum depending on whether you are the winner of your group or not. You can click on as many points on the line as you wish until you find the combination of individual rate and tournament rate that you would like to choose. You can also choose a point on the line by clicking on the buttons labelled with the left and right arrows, which are located below the graph. To confirm your decision, click the 'Submit' button on the bottom right part of your screen.

[Page 12]

\section{TASK 4}

In this task, you earn money by answering the following question: How likely do you think it is that you are the winner of your group in Task 3? Your answer can go from 0 (meaning you are completely certain that you are not the winner of your group) to 100 (meaning you are completely certain that you are the winner of your group). Your earnings in Task 4 can be either $€ 0$ or $€ 20$. The probability of getting $€ 20$ depends on two things:

1. The actual outcome (whether you are the winner or not in your group)

2. The likelihood you pick as an answer to the question above. 
The closer the likelihood you choose is to your actual outcome in Task 3, the higher the probability you have of winning $€ 20$. In other words, if it turns out that you are the tournament winner in Task 3, then the probability that you earn $€ 20$ increases the closer your selected likelihood is to 100 . Conversely, if it turns out that you are not the tournament winner in Task 3, then the probability that you earn $€ 20$ increases the closer your selected likelihood is to 0 .

You will select your likelihood of being the winner of your group by moving the cursor on a slider. You can select any number between 0 and 100. The cursor will appear on the slider only after you have clicked on the slider for the first time. To help you to understand the consequences of your choice, you will see the following information below the slider.

- On the right part of the screen, a table shows the probability of getting $€ 20$ in the two possible outcomes: in case you are the winner in your group and in case you are not the winner in your group.

- On the left part of the screen, a bar graph shows the corresponding expected earnings in both outcomes.

We provide an example below to better illustrate how this information will look like (note that the numbers used in this example are not indicative of what constitutes a good or bad answer in this task). 


\section{Example}

Suppose you think there is an $80 \%$ chance that you are the winner in your group and you selected 80 in slider, as shown in the figure below. Given your choice on the slider the probability of getting $€ 20$ is 0.96 if you turn out to be the winner of your group in Task 3 , and 0.36 if you turn out to be one of the losers. The bar graph shows the corresponding expected earnings: 19.2 if you turn out to be the winner and 7.2 if you turn out to be one of the losers.

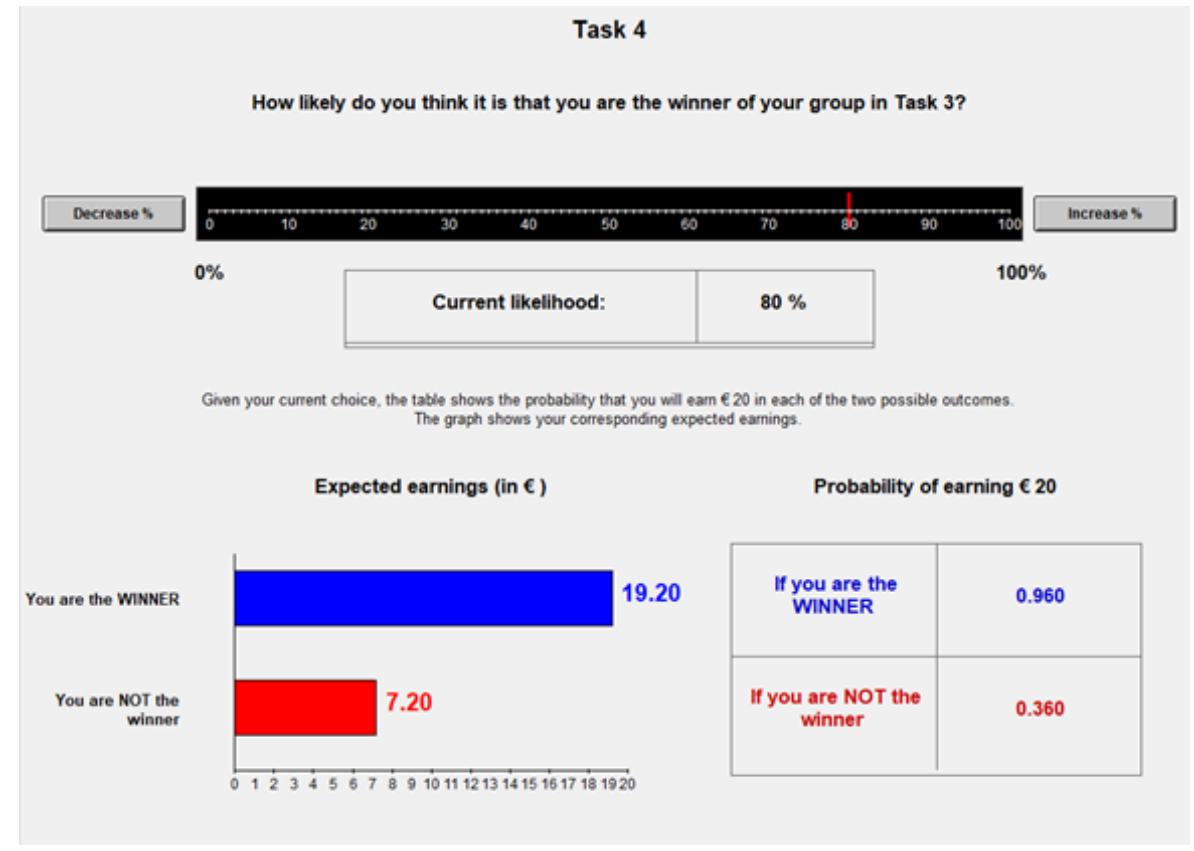

Figure 3.28: Example Task 4

Once you are done reading, click on the "Next" button on your screen. 


\section{[Page 14]}

\section{TASK 5}

This is Task 5 of the experiment. The earnings from this part of the experiment are completely independent from the ones obtained before. The amount you earn depends on your decisions and on chance, that is the outcome of a die you will role at the end of the experiment in case Task 5 is chosen for payment purposes.

In Task 5 you choose how you want to be paid: you choose a combination of a certain amount and a probabilistic amount. Please notice that in Task 5 you don't have to perform any summation task. To make your choice, you pick a point on a line in a graph like the one below. The certain amount corresponds to the horizontal axis, and the probabilistic amount corresponds to the vertical axis. Every point on the blue line resembles a combination of a certain amount and a probabilistic amount. You can only choose one point on the blue line, but you are free to choose any point.

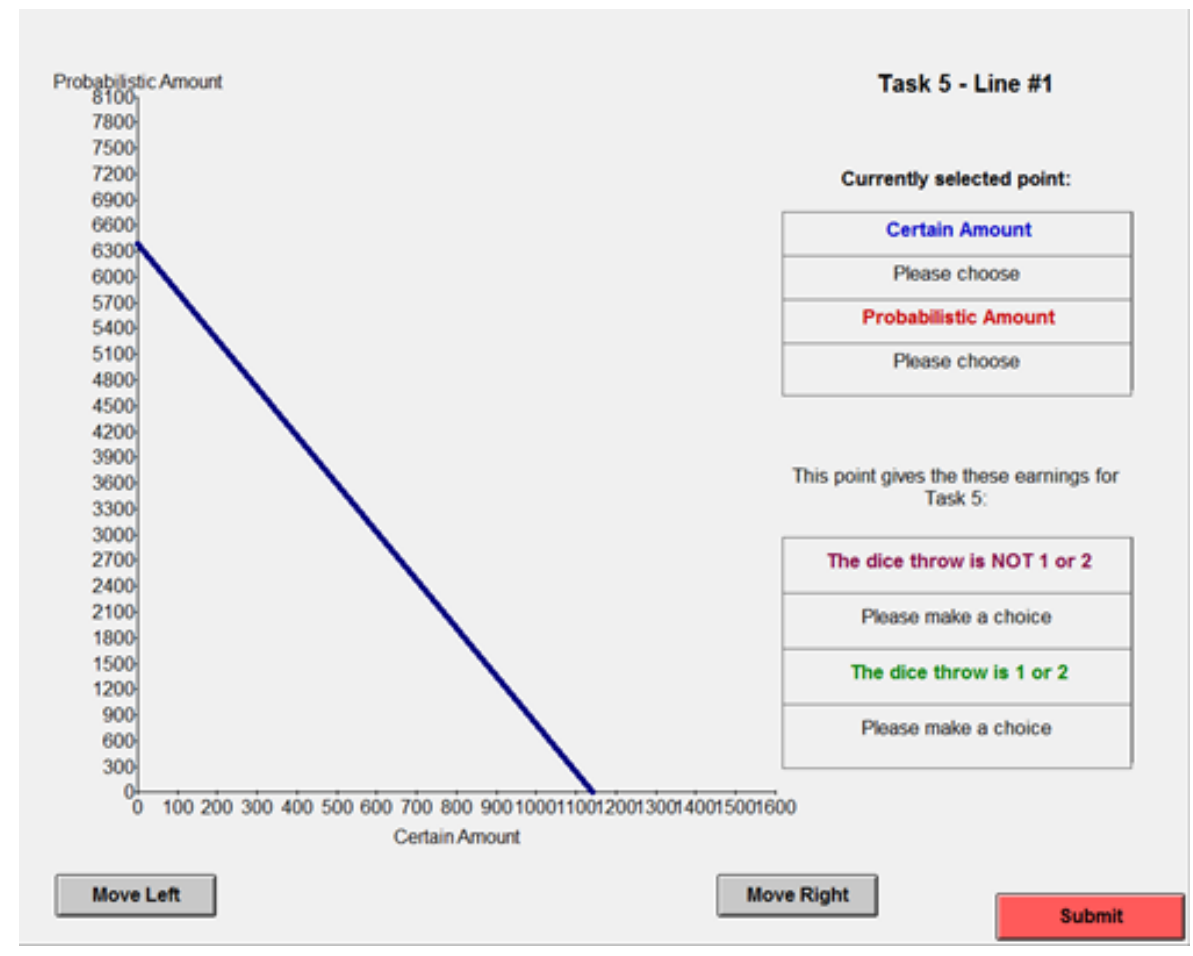

Figure 3.29: Example Task 5

The two options are as follows:

A. The certain amount pays the amount corresponding to the point you choose on the blue line for sure. For example, if you choose a certain amount of 100 then you will receive 100 Tokens.

B. The probabilistic amount pays the amount corresponding to the point 
you choose on the blue line with a probability of $20 \%$. At the end of the experiment, you will throw a ten-sided die. If the outcome of your dice throw is 1 or 2 , you get the probabilistic amount you choose; otherwise you will be paid zero. For example, if you choose a probabilistic amount of 200 then you will receive 200 Tokens if your dice throw results in 1 or 2 . If your dice throw is 3 through 10 , you will receive 0 Tokens.

In summary, you will be paid in the following way in Task 4:

- If the outcome from your ten-dice throw at the end of the experiment is different from 1 or 2:

(Your chosen certain amount)

- If the outcome from your ten-dice throw at the end of the experiment is either 1 or 2 :

(Your chosen certain amount) + (Your chosen probabilistic amount) 


\section{Examples:}

Example 1: Imagine you chose point $A$ in the graph below. In this point, you have a certain amount of 600 Tokens and a probabilistic amount of 1670 Tokens with $20 \%$ chance. This means that:

- If your dice throw is different from 1 or 2 , you earn 600 Tokens: 600 Tokens from your certain amount and 0 Tokens $\mathrm{s}$ from your probabilistic amount.

- If your dice throw is equal to 1 or 2 , you earn 2270 Tokens: 600 Tokens from your certain amount and 1670 Tokens from your probabilistic amount.

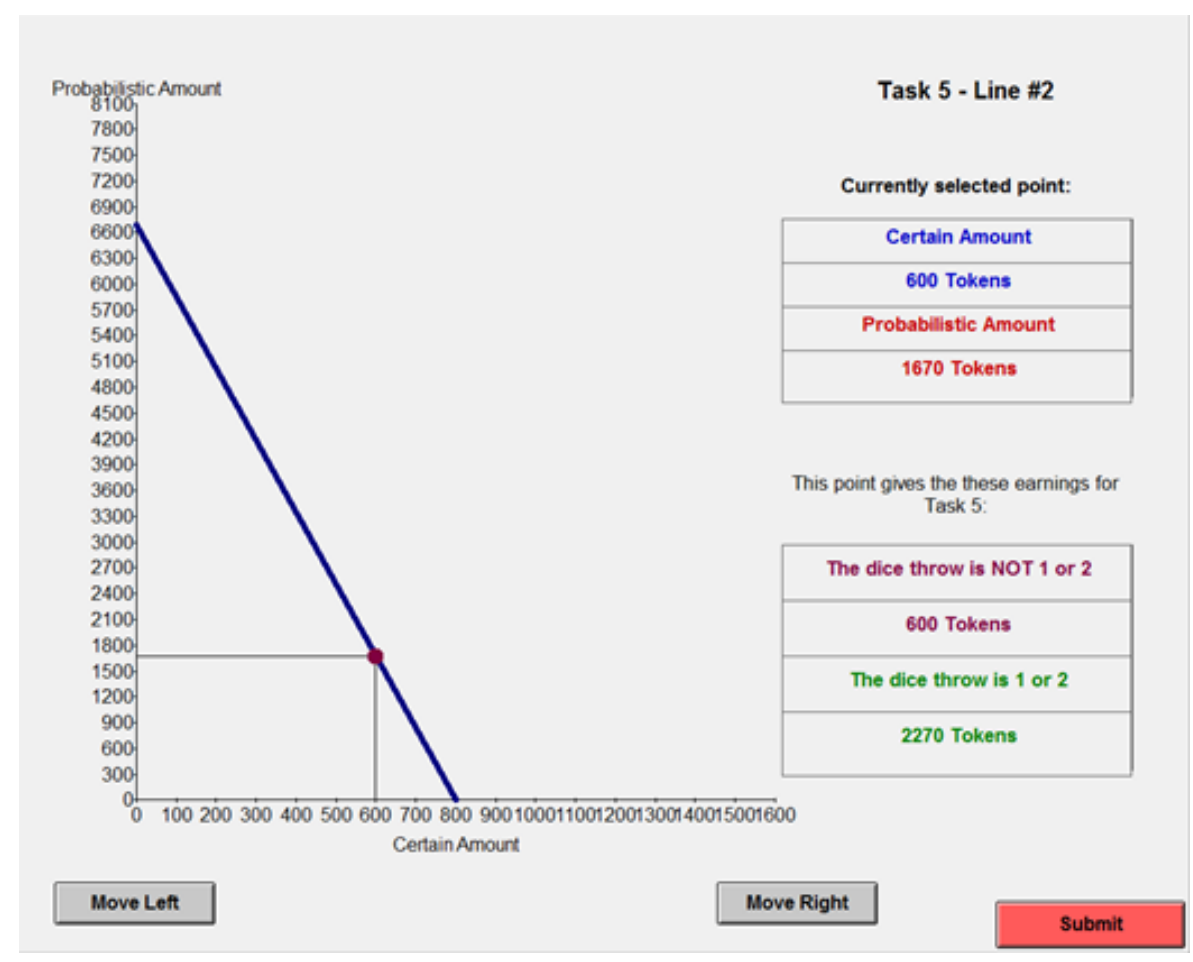

Figure 3.30: Example Task 5

Example 2: Another possible choice is B. In this point, you have a certain amount of 270Tokens and a probabilistic amount of 5400 Tokens. This means that:

- If your dice throw is different from 1 or 2, you earn 270 Tokens: 270 Tokens from your certain amount and 0 Tokens from your probabilistic amount.

- If your dice throw is equal to 1 or 2 , you earn 5670 Tokens: 270 Tokens from your certain amount and 5400 Tokens from your probabilistic amount. 


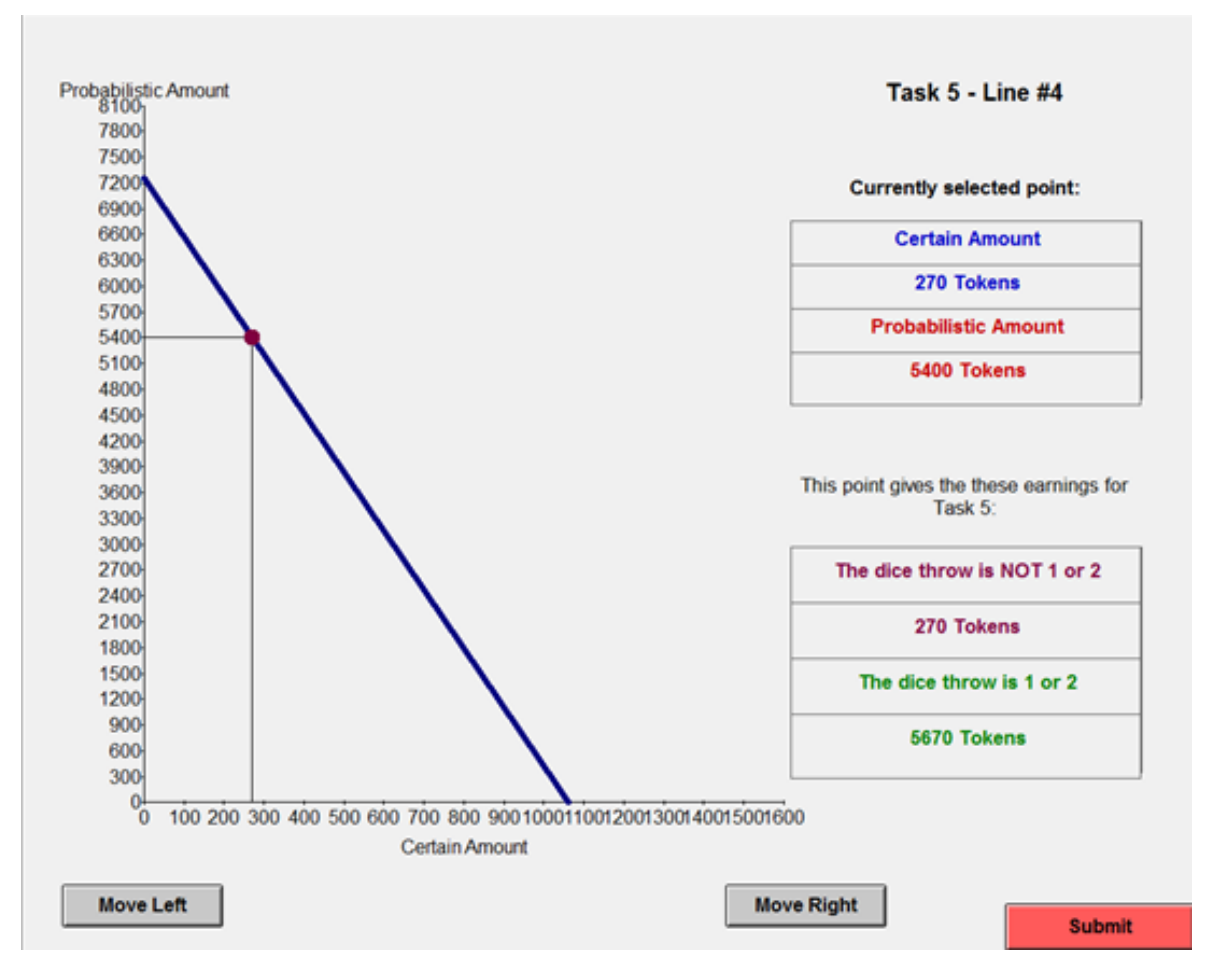

Figure 3.31: Example Task 5

Example 3: Another possible choice is C. In this point, you have a certain amount of 0 Tokens and a probabilistic amount of 3910 Tokens. This means that:

- If your dice throw is different from 1 or 2 , you earn 0 Tokens: 0 Tokens from your certain amount and 0 Tokens from your probabilistic amount.

- If your dice throw is equal to 1 or 2 , you earn 3910 Tokens: 0 Tokens from your certain amount and 3910 Tokens from your probabilistic amount.

You will be asked to make a choice in 40 different lines. The blue line representing the combinations of certain amount and probabilistic amount will change from choice to choice. Examples of lines that you might see are shown below. For each choice, the computer randomly selects a line that crosses the horizontal axis between 500 and 1500 Tokens and the vertical axis between 3000 and 8000 Tokens. After you have made a choice for all your 40 lines, one of them will be randomly selected to determine your earnings for Task 5 (each line with equal probability). If Task 5 is the task randomly selected for payment, the selected choice will be used to calculate your final earnings.

To make a choice, use your mouse to click on a point on the line. Once you click, you will see on the horizontal axis the value for certain amount and on the vertical axis that value for probabilistic amount. On the right side of the screen, you will also see two tables. The table on the top will display the 


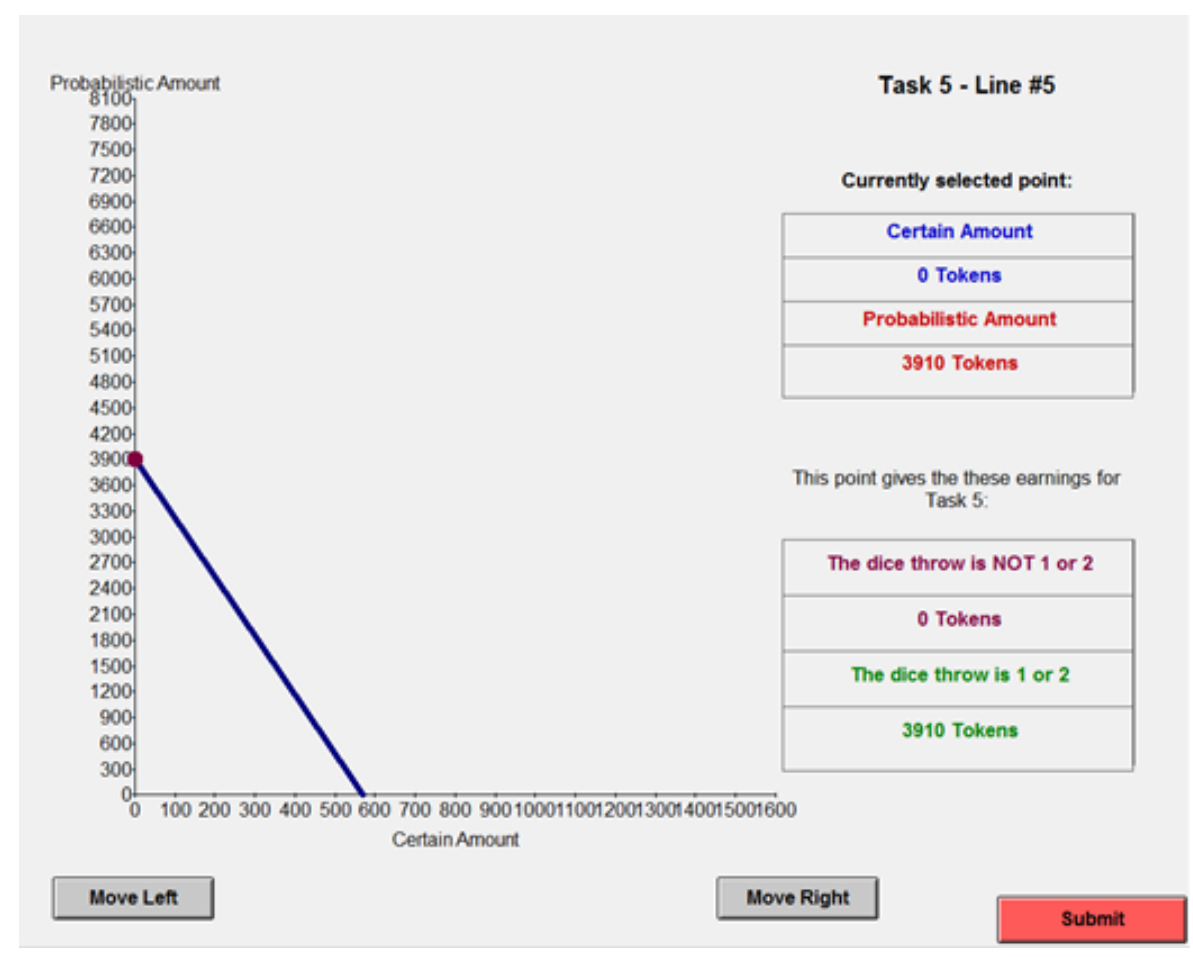

Figure 3.32: Example Task 5.4
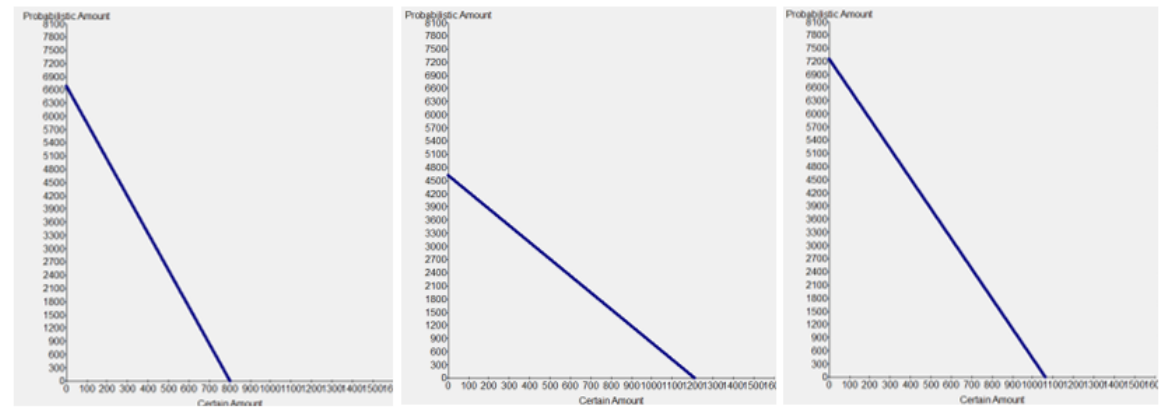

Figure 3.33: Example Task 5

point you selected on the blue line. The table on the bottom will display your earnings (in Tokens) in Task 5 depending on whether your dice throw is equal to 1 or 2 or not. You can click on as many points on the line as you wish until you find the combination of certain amount and probabilistic amount that you would like to choose. You can also choose a point on the line by clicking on the buttons labelled with the left and right arrows, which are located below the graph. To confirm your decision, click the 'Submit' button on the bottom right part of your screen. 


\section{Chapter 4}

\section{The Impact of the Menstrual Cycle on Bargaining Behavior}

In this paper, we examine whether the menstrual cycle influences women's bargaining behavior and bargaining outcomes. In an experiment, participants play a variation of the dynamic unstructured bilateral bargaining game with private information (Camerer et al., 2019). While the distribution of the pie size is common knowledge, only one bargaining partner (informed) knows the actual pie size. Our findings suggest that information plays an important role for the effect of the menstrual cycle on bargaining behavior. Bargaining behavior of less informed women (uninformed) does not vary significantly over the menstrual cycle, but the bargaining behavior of informed women does: During the ovulation phase, informed women offer a smaller share of the pie to the bargaining partner compared to the other menstrual phases, and especially compared to the premenstrual phase. These differences also translate into bargaining outcomes resulting in significantly higher final payoffs for informed women during the ovulation phase. Although the bargaining behavior of uninformed women does not change over the menstrual cycle, their bargaining outcomes captured by final payoffs and deal rates improve during ovulation.

This chapter is based on joint work with Arno Riedl and Christina Rott 


\section{Introduction}

Bargaining is an important part of human interaction and affects greatly individuals' outcomes in both the labor market and the private domain. A disadvantageous position in a negotiation environment can have a tremendous consequence on bargainers' daily life outcomes (e.g. promotions and salaries). For instance, not negotiating the first job offer leads to a $\$ 500,000$ loss throughout women's career in comparison to their male counterparts (Babcock and Laschever, 2009). Understanding the determinants of bargaining behavior and outcomes is therefore of utmost importance.

Bargainers' preferences, as well as the context in which negotiations take place, are important determinants of behavior and outcomes. The literature in experimental and behavioral economics has identified different factors that influence bargaining behavior, such as the sex of the partner with which individuals are negotiating (Eckel et al., 2008), the stereotypes present during the negotiation (Kray et al., 2001), the ambiguity of the situation (Small et al., 2007; Leibbrandt and List, 2015), the framing of the negotiation (Small et al., 2007), the relative positional power (Andersen et al., 2018; Dittrich et al., 2014), and also attitudes towards risk and fairness might contribute to the differences in negotiation outcomes as well (Niederle and Vesterlund, 2007).

In addition to these bargaining determinants, there are other factors understudied in a bargaining context that have a strong connection with individual decision-making processes in an economic domain. Specifically, research in endocrinology has increased our understanding of biological factors underlying different economic outcomes (for a survey see Blau and Kahn (2000)). However, the role of nature in shaping individuals' bargaining behavior is intriguing, but a largely open question. Our study contributes to answering this question by analyzing the bargaining behavior of women across their menstrual cycle.

Understanding the impact of the menstrual cycle on bargaining behavior is important for three reasons. First, the obtained results give insights as to whether nature in the form of the menstrual cycle directly affects negotiation behavior. Second, it increases our understanding of whether other factors such as risk and social preferences have a mediating effect on the menstrual cycle on bargaining behavior. Third, raising women's awareness of variations in bargaining behavior over the menstrual cycle might influence their bargaining behavior positively and thereby reduce gender differences in bargaining outcomes in the labor market.

The menstrual cycle is characterized by the levels of two female sex hormones linked to fertility, i.e., estradiol and progesterone, that fluctuate in a predictable pattern and constantly influence women's behavior. In the last two decades, the gender literature in economics has suggested a connection between the menstrual cycle and female's decision-making process in multiple domains. To date, the impact of the menstrual cycle on economic decision making has been studied in the context of sealed-bid first-price auctions (Chen et al., 2013; Pearson and Schipper, 2013), competitiveness (Wozniak et al., 2009; Buser, 2012; Ranehill et al., 2018), risk preferences (Buser, 2012; 
Ranehill et al., 2018), altruism (Buser, 2011; Ranehill et al., 2018), loss aversion, and economic rationality (Lazzaro et al., 2016). However, to the best of our knowledge, the possible link between biological factors and decisions has been ignored in the context of bargaining.

One main contribution of our paper is to provide evidence of the influence of the menstrual cycle on bargaining behavior and outcomes. The menstrual cycle is a promising determinant of bargaining as it has been shown to influence different types of behaviors and preferences that are present in a negotiation context such as women's risk preferences and loss aversion (Buser, 2012; Lazzaro et al., 2016), social preferences (Buser, 2011), and competitiveness levels (Wozniak et al., 2009; Buser, 2012). This literature together suggests that the menstrual cycle, and more specifically the female sex hormones related to fertility, influence women's economic preferences in those domains.

In this study, we follow Camerer et al. (2019)'s design and implement a negotiation environment with a bilateral unstructured bargaining scheme characterized by one-side private information between the involved parts. In the bargaining game, two individuals bargain over the allocation of an amount of money (i.e., the pie size). While the distribution of the pie size is common knowledge, only one bargaining partner (informed) knows the actual pie size whereas the other partner does not have access to this information (uninformed). Following the bargaining game, risk and social preferences are elicited. Before coming to the bargaining experiment phase, subjects are asked to track their menstrual cycle for three months. This self-reported information allows us to obtain an accurate measurement at the individual level of the cycle length and the menstrual phase of each woman during the bargaining experiment phase.

This paper proposes that the hormonal changes present during women's menstrual cycle, mainly due to progesterone and estradiol levels, influence bargaining outcomes and consequences. Following the evidence that suggests that women are competitive, less risk averse and profit-maximizing when they face drastic changes in estradiol levels during ovulation (e.g. Wozniak et al. (2009), Buser (2012), Lazzaro et al. (2016)), we hypothesize the following two outcomes. First, that bargaining behavior is related to fluctuations of sex hormones, and therefore, to the menstrual cycle. Second, that women's bargaining behavior is less compromising during the ovulation phase compared to the other phases and, especially, compared to the premenstruation phase. We also expect variation in bargaining consequences, which we capture by looking at changes in deal rates and final payoffs across the menstrual cycle. Specifically, we expect that a decrease in compromising behavior during the ovulation phase is translated into higher final payoffs and lower deal rates during the ovulation in comparison to the other three menstrual phases.

Our main findings are as follows. Supporting our second and main hypothesis, we observe that bargaining behavior varies over the menstrual cycle, and this variation is conditional on the information setting. Specifically, when women are not affected by any asymmetry of information (informed), 
they are less willing to compromise in our bargaining setting during the ovulation phase in comparison to all the other menstrual phases, and this effect is strongest when compared to the premenstruation phase. Interestingly, this effect is independent of risk and social preferences. We also observe that the decrease in compromising behavior during the ovulation phase versus the premenstruation phase is translated into higher payoffs during the former phase in comparison to the later one. Lastly, although we do not observe variation in bargaining behavior across the menstrual cycle for women exposed to asymmetry in information (uninformed), we do see an increase in final payoffs and deal rates during the ovulation phase for these players. Taking together, our findings suggest that the effect of the menstrual cycle on bargaining behavior is conditional on the information setting. In addition, they point out the important role of ovulation and premenstrual phases as determinants of women's bargaining behavior and consequences.

The remainder of this chapter is organized as follows. We provide a literature review in Section 2. Section 3 describes the experimental design and how we estimated the menstrual cycle phases for our sample. We describe our hypotheses in Section 4. In Section 5, we present our results and in Section 6 we discuss them. Finally, we conclude in Section 7.

\section{Literature review}

Evidence of the impact of the menstrual cycle on preferences and economic decision-making is somewhat mixed, but overall points towards the important roles of two menstrual phases: the ovulation and premenstruation (or luteal) phases. In general, previous work suggests that the menstrual cycle, and more specifically, the hormones associated with fertility and reproduction, might influence economic preferences and behavior. We focus on competitive behavior, risk aversion, and social preferences; given that these are the main aspects present in a bargaining environment that have been studied in relation to the menstrual cycle.

In the context of competition, there are three studies that look at the variations in competitive behavior over the menstrual cycle by using the experimental task proposed by Niederle and Vesterlund (2007). In a first study, Wozniak et al. (2009) examines whether women have between and within differences in competition choices during the menstrual cycle with self-reported data. Their findings suggest that women's willingness to select into a competitive environment is higher when progesterone levels are high. In a second study, Buser (2012) reveals an opposite pattern to the one observed in Wozniak et al. (2009). Buser (2012) uses self-reported menstrual cycle data to show that women's willingness to select into a competitive environment changes over the menstrual cycle and also with the intake of hormonal contraceptives. His results suggest that women are less competitive when the levels of progesterone are high. That is, during the premenstruation phase or during the 21-days intake period for hormonal contraceptive takers. Additionally, his findings suggest that such variation in competitive behavior over the menstrual cycle is not explained by other factors such as 
performance, risk aversion, or overconfidence. It is worth mentioning that it is difficult to compare both studies because both competitive tasks differ and each study has a different gender composition in its sample. Buser (2012) uses a all-female sample whereas Wozniak et al. (2009) has mixed-sex sample. Lastly, a recent study Ranehill et al. (2018) employs a clinical randomized placebo-controlled method to study the causal effect of hormonal contraceptives on risk, social, and competitive preferences. Their results suggest a null effect of hormonal contraceptives, and probably also the female sex hormones present during the menstrual cycle, on any of these three economic preferences, including competitiveness.

In the context of risky behavior, Buser (2012), Ranehill et al. (2018) and Schipper (2014) find no variation of risk attitudes during the menstrual cycle, whereas Lazzaro et al. (2016) observe a reduction in risk aversion during the ovulation phase when compared to the luteal phase, but not to the other phases. Bröder and Hohmann (2003) and Chavanne and Gallup Jr (1998) also observe an increase in risk-taking behavior during the mid part of the menstrual cycle (i.e., during ovulation). However, these last two studies specifically look at behaviors that increase the risk of falling victim to rape and not at general risk preferences. For social preferences, Buser (2011) find higher levels of trust before and during ovulation and higher levels of altruism during the premenstrual phase. Contrary, Ranehill et al. (2018) do not find any differences in altruistic behavior during the menstrual cycle.

The menstrual cycle has also been suggested to influence behavior in two other economic domains: competitive bidding and loss aversion. For sealed bid first-price auctions, Chen et al. (2013) and Pearson and Schipper (2014) observe that women bid lower when hormone levels are high (i.e., during the premenstrual phase). In a setting of loss aversion, Lazzaro et al. (2016) demonstrates that women are less loss averse and more willing to take risky options that may lead to potential losses compared to men during the ovulation phase.

It is difficult to compare results between studies in this literature for several reasons. First, most of them use different methods to estimate the menstrual cycle phases. In some studies, self-reported data are used whereas in others, biological measurements are employed to estimate the menstrual phases or a combination of both methods. Also, across studies, the division of the menstrual cycle varies (from two to five phases), as well as the sample composition (all-female or mixed sex). Each of these factors can lead to differences in outcomes and conclusions. However, most of the studies on this literature seem to have a consensus on the important role of the premenstruation and ovulation phases on economic behaviors, as those two menstrual phases face the most drastic changes in estradiol and progesterone levels (Wozniak et al., 2009; Buser, 2012; Lazzaro et al., 2016).

\section{Experimental design and procedures}

Participants in our study are invited to a lab session after a period of tracking their menstrual cycle. In the lab session, participants play a dynamic 
unstructured bargaining game. To be able to identify women's cycle phase at the individual level at the moment of the experimental session, women are recruited three months earlier and asked to track their menstrual cycle until the session. In the following two subsections, we describe the experimental sessions with the bargaining game and the estimation of the cycle phase, respectively.

\subsection{Experimental sessions with bargaining game}

Each experimental session is divided into four parts: In Part 1, participants play a dynamic unstructured bargaining game, in Part 2 and 3, we elicit risk and social preferences, and in Part 4, participants complete a demographic questionnaire. The instructions for each part are provided at the beginning of the respective part and can be found in Appendix A6.

\section{Part 1: Bargaining game}

In part 1, participants play a variation of the continuous-time bargaining game with one-sided private information developed by Camerer et al. (2019). Two players bargain over the allocation of an amount of money, which we refer to as the pie size. The distribution of the pie sizes $(€ 4.00, € 8.00$, $€ 12.00$, $€ 16.00$, $€ 20.00$, or $€ 24.00$ ) is public knowledge as well as is the fact that the computer picks a pie size with equal probability. Only one of the two players - the informed player-knows the actual pie size. The other player-the uninformed player-only knows the distribution of the potential pie sizes. Both players know that only the informed player is informed about the actual pie size. At the beginning of the experiment, players are randomly assigned the role of an informed player or uninformed player and keep the assigned role for the entire experiment. Participants are assigned to 28 independent matching groups composed of three informed players and three uninformed players, respectively.

The randomly paired players negotiate over the uninformed player's share of the pie. Since players bargain over the uninformed player's share of the pie, we refer to the informed player's bargaining proposals as offers and the uninformed player's bargaining proposals as demands. Players can place offers or demands on a slider interface. The bargaining positions can take any value between $€ 0.00$ and $€ 24.00$ in multiples of $€ 0.20$.

The bargaining game consists of an initial bargaining stage, a simultaneous bargaining stage, and a feedback stage. Screenshots of the bargaining stages are displayed in Appendix A6.

Stage 1. Initial bargaining: Both players indicate their initial offer/ demand without observing the other player's bargaining position. ${ }^{1}$ Once both players indicated and submitted their initial bargaining position, each player's initial position is revealed to the paired player by displaying the informed and

\footnotetext{
${ }^{1}$ The initial cursor location on the slider (i.e., before the first click) is randomized.
} 
the uninformed player's sliders right below each other on the screen. In this stage, no time constraint is imposed. However, players can only move to the next stage after having submitted their initial offer/demand.

Stage 2. Continuous bargaining: After observing the paired player's initial position, participants have 30 seconds to bargain and reach a deal. Offers and demands can be made continuously at any time within the time frame. Players see their bargaining partner's current offer/demand immediately and simultaneously on the slider. A deal is reached and the continuous bargaining stage terminates if offer and demand coincide and do not change for two seconds. If a deal is reached, the uninformed player's payoff is equal to the agreed share and the informed player's payoff is equal to the pie size minus the agreed share for the uninformed player. In case the agreed share for the uninformed player exceeds the pie size, the informed player's payoff is negative. Negative payoffs are subtracted from other earnings in the experiment. If no deal is reached after 30 seconds of bargaining, both players get $€ 0.00$.

Stage 3. Feedback: After the bargaining game, both players are informed of their own payoff and the actual pie size.

Participants play the game repeatedly for ten rounds and bargaining partners are paired in matching groups in a strangers protocol. ${ }^{2}$ At the end of the experiment, one of the ten bargaining rounds is randomly selected for payment.

\section{Part 2 : Risk preferences}

In part 2, participants' risk preferences are elicited following the experimental design in Cettolin et al. (2017). For each of the ten lotteries displayed in Table 4.1, participants make a choice between the lottery and a sure amount. ${ }^{3}$ Specifically, the sure amounts decrease monotonically from the highest outcome in the lottery to the lowest outcome in the lottery in equally spaced steps. To ensure a unique switching point, subjects are allowed to switch only once between the lottery and the sure amount.

We calculate the participants' certainty equivalents of ten multiple price lists (MPL) with 10 two-outcome lotteries within each MPL, and use them to estimate each participant's risk preferences. Risk preferences are calculated at the individual level assuming a CRRA power utility function for money $U(x)=x^{1-\alpha}$, where $0<\alpha<1$ indicates risk aversion, $\alpha=0$ risk neutrality and $\alpha<0$ risk-loving (Holt and Laury, 2002; Andersen et al., 2008; Wakker, 2008; Dohmen et al., 2011). At the end of the experiment, one of the 100 decisions is selected with equal probability to be paid out.

\footnotetext{
${ }^{2}$ Participants are randomly matched with a new participants within their matching group. The probability to interact with the same participant is non-zero but participants never received feedback on this matter.

${ }^{3}$ see Appendix A6 for an example of the screens subjects are displayed during the experiment.
} 
Table 4.1: Lotteries for risk preferences

\begin{tabular}{cccc}
\hline Lottery & $p 1$ & $x 1$ & $x 2$ \\
\hline 1 & 0.2 & 16 & 5 \\
2 & 0.5 & 10 & 4 \\
3 & 0.8 & 7 & 20 \\
4 & 0.15 & 22 & 2 \\
5 & 0.25 & 15 & 4 \\
6 & 0.33 & 12 & 0 \\
7 & 0.2 & 13 & 3 \\
8 & 0.35 & 18 & 6 \\
9 & 0.9 & 4 & 24 \\
10 & 0.33 & 10 & 6 \\
\hline
\end{tabular}

Note: $p 1$ is the probability of winning $x 1 €$.

\section{Part 3: Social preferences}

In part 3, we elicit social preferences by means of the experimental task developed by Kerschbamer (2015). In this task, participants make incentivized binary choices between the allocation of payoffs to the decision-maker and to a randomly matched player - the passive player. Participants have to choose between an equal split for themselves and the passive player and an allocation with (dis-)advantageous inequality. A detailed description of the elicitation of social preferences is provided in the Appendix A1.

We create an index that measures the degree of benevolent (or malevolent) behavior given the choices subjects make among each of the two types of decisions. Specifically, we categorized a player with a high degree of benevolence if when confronted with the binary choices in the disadvantageous inequality block, he or she chooses most of the time the asymmetric allocation; and when confronted with the binary choices in the advantageous inequality block, he or she chooses most of the time the symmetric allocation. ${ }^{4}$ At the end of the experiment, one of the choices is selected randomly and the participants receive the payment as active decision-maker in that choice plus the payoffs obtained as a passive player. $^{5}$

\footnotetext{
${ }^{4}$ Note that we do not use the measurement of distributional preferences suggested by Kerschbamer (2015) because the switching behavior of our subjects doesn't allow for the rationalization of such choices. We have more than one switching per block for some subjects. This inconsistent switching behavior makes it difficult to estimate subjects' distributional preferences.

${ }^{5}$ Following the design of Kerschbamer (2015), we used a double role assignment protocol. That is, each subject gets two payoffs in this task, one obtained for the role of decision-maker and the other one for the role of the passive player. At the end of the experiment, the earnings from this task correspond to the payoffs from one of the ten choices made as an active decisionmaker that is randomly selected with equal probability plus the payoffs obtained as a passive player.
} 


\section{Part 4: Questionnaire}

In part 4, participants fill in a questionnaire, which includes socio-demographic questions on gender, age, nationality, and study level. Additionally, they provide information about their menstrual cycle to verify the data obtained from their prior menstrual cycle tracking. Using the same questionnaire as in Buser (2012), participants are asked to indicate the date when the last menstruation started, their average cycle length, the average length of menstruation, whether they are experiencing menstrual bleeding at the moment of the laboratory session, how regular their cycle is, and whether they use a hormonal contraceptive method (and if so which one). We also ask questions about their risk attitudes in different contexts following the design of Dohmen et al. (2011).

\subsection{Tracking of the menstrual cycle and estimation of cycle phase}

According to the medical literature, the menstrual cycle is mainly characterized by two female sex hormones-oestrogen (or estradiol) and progesterone (Stricker et al., 2006). Among non-contraceptive takers, these hormones fluctuate in a predictable pattern during an average cycle length of 28 days (Haag et al., 2016). The individual cycle length varies however greatly across women. As displayed in Figure 4.1, the natural menstrual cycle can be divided into four phases: menstrual, postmenstrual (or follicular), ovulation, and premenstrual (or luteal) phase.

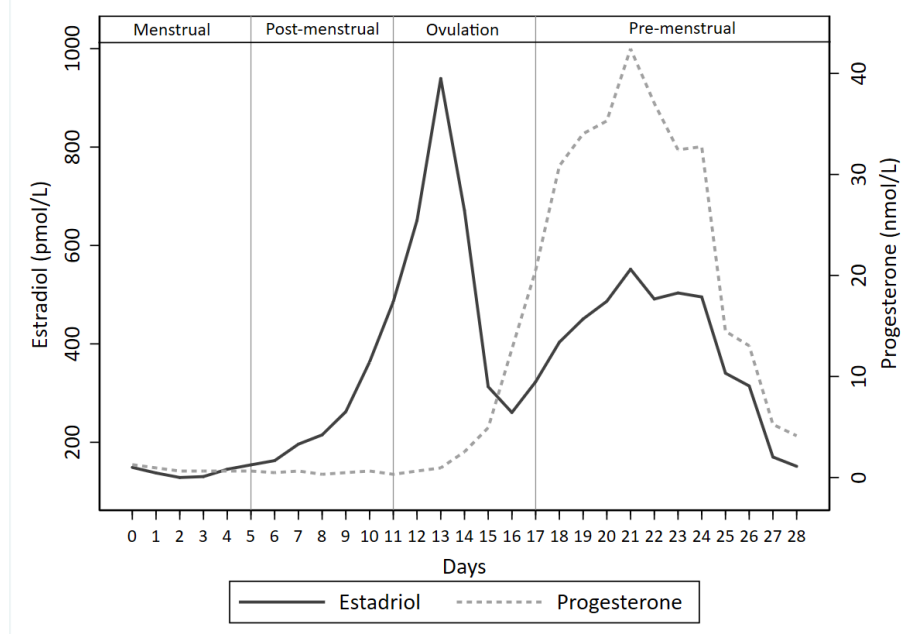

Figure 4.1: Menstrual phases and hormone levels over the menstrual cycle.

Note: Menstrual cycle division suggested by the gynecological text Haag et al. (2016).

For hormonal contraceptive takers, the hormones fluctuate in a different pattern than the one observed in non-hormonal contraceptive women (Lovett et al., 2017). The most common types of hormonal contraceptives influence the natural cycle of women through a 21-day intake period, during which daily artificial doses of estradiol and progestin increase the levels of estradiol and 
progesterone (Golobof and Kiley, 2016; Christin-Maitre, 2013). ${ }^{6}$ The 21-day intake period is followed by a 7-day break period without pill intake, where the hormone levels decline substantially (Buser, 2012). According to Lovett et al. (2017), contraceptive takers experience very different hormone levels during the 28-day cycle than non-contraceptive takers and the hormone levels vary considerably across contraceptives. ${ }^{7}$ Contraceptives also affect other biological aspects: Conditional on a correct intake of the hormonal contraceptives and no disrupting factors, contraceptive takers are not fertile (during the naturally occurring ovulation phase). We, therefore, estimate the menstrual phases only for non-contraceptive takers.

After the three-month tracking period, we obtain a reliable measurement of the cycle length and the menstrual phase at the individual level. The mean cycle length is 30 days (standard deviation: 4.33 days) and varies between 21 and 46 days. We follow a similar approach as Buser (2012) and Lazzaro et al. (2016) and use the participants' reported start date of their last menstruation and their individual average cycle length to estimate the first day of their next menstruation. Since the duration of ovulation and the premenstrual phase are relatively constant, we calculate backward the dates of each of the four menstrual phases (postmenstrual, ovulation, and premenstrual). This gives us an individual estimate of the menstrual phase in which participants are when they come to the laboratory session. In Appendix A1, we explain in detail how the menstrual phases are obtained at the individual level in our sample.

In this study, we measure the menstrual cycle phases with self-reported data. ${ }^{8}$ We account for potential measurement errors in our menstrual phase estimation in three ways: First, we ask participants to track their menstrual cycle for a period of three months. As suggested by the medical literature (Lazzaro et al., 2016), a three months period is sufficient to have a measurement of the menstrual cycle length and variation at the individual level. Thus, it allows us to control for individual variations in the cycle length. Second, after the three months tracking period participants are asked in the questionnaire at the end of the lab session to report when their last menstruation started. We compare this last menstruation information with the

\footnotetext{
${ }^{6}$ Progestin is the name of a synthetic hormone with a similar effect as the progesterone hormone.

${ }^{7}$ Lovett et al. (2017) analyze the hormonal levels of US women who take one of the seven most common contraceptive pills on the market. Our study was conducted in Europe, but we have no valid reason to expect contraceptives on the European market to differ greatly from those on the US market.

${ }^{8}$ The menstrual phases can be alternatively estimated by measuring women's hormone level through, for instance, saliva or blood samples. This measure might be more reliable (Creinin et al., 2004), but it requires several measurements over a certain period of time because the natural hormone levels vary greatly across women. For instance, the same measurement of progesterone might represent a high level for one woman and be a regular level for another woman. Because of our between-subject design, we decided against a physiological measurement. Additionally, we believe that self-reported data may have a practical advantage over hormonal data in terms of trading-off the accuracy of the menstrual cycle measurement for a more realistic environment: Using self-reported data is less invasive and allows us to mimic better a real-life setting where women have rather access to self-tracking devices (for instance, on their smartphones) than to biological measures to track their own menstrual cycle.
} 
estimation of the menstrual phase obtained from the tracking period. For all participants except two, the two reports are consistent. Third, we run a robustness check by adjusting the definition of the phase duration. Specifically, we reduce the ovulation phase by two days and adjust the other phases accordingly. As reported in Appendix A4, the results do not change with the adjusted measurement.

\subsection{Experimental procedures}

The bargaining experiment was conducted at the Behavioral and Experimental Economics Laboratory (BEElab) at Maastricht University and data were collected in two waves: wave one in January-May 2016 and wave two in January-May 2017. We ran a total of nine experimental sessions. Participants were recruited through the online recruitment system ORSEE (Greiner, 2015). All participants agreed to track their menstrual cycle for three months and were either non-contraceptive or contraceptive takers under a method that did not suppress menstruation completely. ${ }^{9}$ In total, 166 women signed the informed consent and participated in the lab session, where 97 were non-pill takers (with 45 uninformed players and 52 informed players) and 69 were pill takers (with 38 uninformed players and 31 informed players).

The study was approved by the Ethical Review Committee Psychology and Neuroscience (ERCPN) at Maastricht University. Participants received in cash a $€ 10$ bonus at the end of the study for providing their menstrual cycle information plus a $€ 5$ show-up fee and the payment from one of the three parts randomly selected from the bargaining experiment phase. The bargaining session lasted on average 45 minutes and the total average payment was $€ 35$. The experiment was programmed in and executed with the software z-Tree (Fischbacher, 2007).

\section{Hypotheses}

To study the role of the menstrual cycle on bargaining, we look at both individuals' bargaining behavior and the consequences thereof. We focus on non-contraceptive takers as contraceptive takers have large differences in hormonal levels with respect to non-pill takers and they do not experience the same menstrual phases (Lovett et al., 2017). We analyze bargaining behavior in terms of compromising behavior, which is measured by informed players' initial offers, uninformed players' initial demands, and concession rates. We focus on initial offers and demands because they best capture an individual's preferences and beliefs before being affected by the negotiation partner's position. To capture the interactive nature of bargaining, we study participants' concession rates during the simultaneous bargaining stage following the relative concession index developed by Gächter and Riedl

\footnotetext{
${ }^{9}$ The only exclusion criteria was not experiencing a menstrual cycle at all (which can be due to contraceptive methods such as intrauterine devices, pregnancy, or trans-sexuality). In Appendix A1, we describe in detail how participants tracked their menstrual cycle and the information they received for this purpose.
} 
(2005). Concessions reveal a player's willingness to give up part of their own stake to reach an agreement and make the bargaining partner better off. ${ }^{10}$

Bargaining outcomes are important measures of negotiation success. On the individual level, we analyze bargainers' payoffs (both conditional and unconditional on reaching an agreement) and, on the pair level, we compare deal rates among women in different cycle phases. The first hypothesis addresses how differently bargaining behavior of informed and uninformed players is. Camerer et al. (2019) show that informed and uninformed players differ in bargaining behavior in an asymmetric information setting like ours. Specifically, the one-sided private information present in the bargaining setting introduces more uncertainty for the uninformed players and causes risk preferences to be more salient for the players affected by the information asymmetry.

Hypothesis 1 - Bargaining behavior of informed and uninformed players differs.

In addition, we also expect different bargaining behavior across the menstrual cycle for informed and uninformed players. Buser (2012) and Lazzaro et al. (2016) provide some evidence on the role of the menstrual cycle for risk preferences. We, therefore, expect that the information asymmetry moderates the effect of the menstrual cycle and that, consequently, the bargaining behavior of both informed and uninformed players varies across the menstrual cycle. The drastic changes of estradiol levels during ovulation-the fertile phase of the menstrual cycle-have been associated with higher levels of risky, competitive, and profit-maximizing behavior; see, for instance, Wozniak et al. (2009), Buser (2012), and Lazzaro et al. (2016). Translating these effects into a bargaining setting, we expect women to be less willing to compromise during the ovulation phase, that is when estradiol levels are high and facing drastic changes. In addition, we expect a stronger difference in bargaining behavior between the ovulation and the premenstrual phases. The premenstrual phase has been linked with economic behavior that goes in the opposite direction as the one observed during ovulation: High levels of progesterone or drastic changes in progesterone have been shown to reduce risk, antisocial, and competitive behavior (Buser, 2012, 2011; Lazzaro et al., 2016). In addition, from an evolutionary perspective, women engage less in competitive behavior during their infertile phase and pregnancy (i.e., premenstruation phase) than in their fertile phase (i.e., ovulation phase), which is when women compete for the fittest males for reproduction purposes (Lazzaro et al., 2016). Our second and main hypothesis is on the impact of the menstrual cycle on the bargaining behavior of informed and uninformed players.

Hypothesis 2.a - Informed players' initial offers and concession rates are lower

\footnotetext{
5.2 .

${ }^{10}$ For an in-depth description of how the relative concession index is constructed, see Section
} 
during ovulation compared to the other phases, and especially, compared to the premenstrual phase.

Hypothesis 2.b - Uninformed players' initial demands are higher and concession rates lower during ovulation compared to the other phases, and especially, compared to the premenstrual phase.

We expect risk and social preferences to influence women's willingness to compromise during bargaining and, therefore, control for these factors. Risk preferences (Camerer et al., 2019; Embrey et al., 2021) and social preferences (Gächter and Riedl, 2005; Karagözoğlu and Riedl, 2015; Bolton and Karagözoğlu, 2016) are important determinants of bargaining outcomes. We expect risk averse informed players to offer more to the bargaining partner and risk averse uninformed players to demand less. Pro-social individuals are expected to choose more generous allocations, that is, higher offers of informed players and lower demands of uninformed players.

Two important consequences of the negotiation behavior are players' payoffs and deal rates. We expect to observe higher payoffs during the ovulation phase as a consequence of less compromising bargaining behavior-in particular, compared to the premenstrual phase, but only if the less compromising behavior does not lead to lower deals. The extent to which payoffs are higher during the ovulation phase is likely to differ for informed and uninformed players as both players are exposed to different degrees of uncertainty. Deal rates depend on the bargaining behavior of both negotiation partners-informed and uninformed players. In line with the bargaining behavior, we expect lower deal rates if one of the bargaining partners is in the ovulation phase-especially, compared to the premenstrual phase.

\section{Results}

Because of the large differences in hormonal levels between non-contraceptive takers and contraceptive-takers Lovett et al. (2017), we analyze their bargaining behavior and consequences separately. In this section, we report our main analysis and results for only non-contraceptive takers. ${ }^{11}$ The analysis for contraceptive takers is provided in Appendix A5. ${ }^{12}$ We analyze bargaining behavior and consequences with Ordinary Least Square regressions clustering on the matching group level. ${ }^{13}$

\footnotetext{
${ }^{11}$ Importantly, although we do not include contraceptive takers in our main analysis, the interactions these participants had with non-contraceptive takers are kept in the analysis.

${ }^{12}$ In Appendix A2, we provide descriptive statistics of our sample for both contraceptive and non-contraceptive takers.

${ }^{13}$ For the sample of non-contraceptive takers only one participant was excluded (and her corresponding 10 interactions in the bargaining game) from the analysis because she did not complete the menstrual tracking phase. We also have in our sample some non-contraceptive takers with an irregular cycle, but we decided to keep them in our analysis. We define two types of irregularities in our sample. The first one concerns participants with a very long menstrual cycle ( 2 participants have an average cycle longer than 40 days). The second one concerns participants whose calendar report does not match the information provided in the questionnaire they answer in the lab session (2 participants provided conflicting information in that regard,
} 
This section is divided into three sub-sections: first, we compare informed and uninformed players' bargaining behavior across the menstrual cycle (Hypothesis 1). Second, we analyze informed players' offers and uninformed players' demands as well as their respective concession rates (Hypotheses 2a and $2 \mathrm{~b}$ ) and also take into account potential variations of risk and social preferences over the menstrual cycle. Third, we investigate the consequences of bargaining behavior across the menstrual cycle in terms of players' payoffs and deal rates.

\subsection{Comparison of informed and uninformed players}

To test Hypothesis 1, we consider the bargaining behavior of informed and uninformed players and compare their bargaining positions over the duration of the interactive bargaining stage and across menstrual cycle phases. Figure 4.2 displays the mean bargaining positions per second in the continuous and interactive 30-second bargaining stage for informed players (left panel) and uninformed players (right panel). For convenience, the chronological order of informed players' offers is from left to right (left panel) and time passes from right to left for uninformed players' demands (right panel). Each line represents the average position at a given moment in time for one of the four phases of the menstrual cycle (aggregated for all 10 bargaining rounds).

Figure 4.2 shows that informed players' offers start low (mean $€ 4,77$, and $\mathrm{sd}=€ 4.30$ ) and uninformed players' demands start high (mean $€ 11.21$, and $s d=€ 4.77)$. Informed players' offers increase and uninformed players' demands decline over time eventually converging towards the end of the continuous bargaining stage. The observed pattern is very similar to the bargaining dynamics found in Camerer et al. (2019). An agreement is reached in $69 \%$ and $71 \%$ of the cases for informed players and uninformed players, respectively. ${ }^{14}$ We observe a significant strong positive relationship between the bargaining time and the informed player's bargaining position and a strong negative relationship between the bargaining time and the uninformed player's bargaining position (Zellner's seemingly unrelated regression $p<0.001$ and $p<0.001$, respectively). ${ }^{15}$ In addition, we also observe

\footnotetext{
but we decided to keep the one obtained from their tracking calendar).

${ }^{14}$ Note that in principle the deal rates for both type of players should be the same as if one reaches a deal the other also does. However, for the informed (uninformed) players' deal rates we only consider informed (uninformed) players that are non-contraceptive takers. Since the distribution of non-contraceptive takers is different between the two types of players $(n=52$ for informed players and $n=45$ for uninformed players), we have different number of observations for informed and uninformed players. In consequence, we have different deal rates for both type of players. In Table 4.6 we present in detail the deal rates for informed players and uninformed players across the four menstrual phases.

${ }^{15}$ Since the steepness relationship between the bargaining time and bargaining position of informed and uninformed players might be correlated due to their interactions, we use a Zellner's seemingly unrelated regression that accounts for correlated error terms in both relationships. In this regression, we use the bargaining position as a dependent variable and the time in seconds as an explanatory variable for informed and uninformed players, separately. The resulting coefficient estimate for the informed player is $0.046(S E=0.001)$. For the uninformed player, the resulting coefficient estimate is $-0.177(S E=0.006)$.
} 
significant differences in the steepness of the slope for the bargaining position across time between informed and uninformed players (post-estimation Wald test of the absolute coefficients obtained from the Zellner's seemingly unrelated regression, $p<0.001) .{ }^{16}$

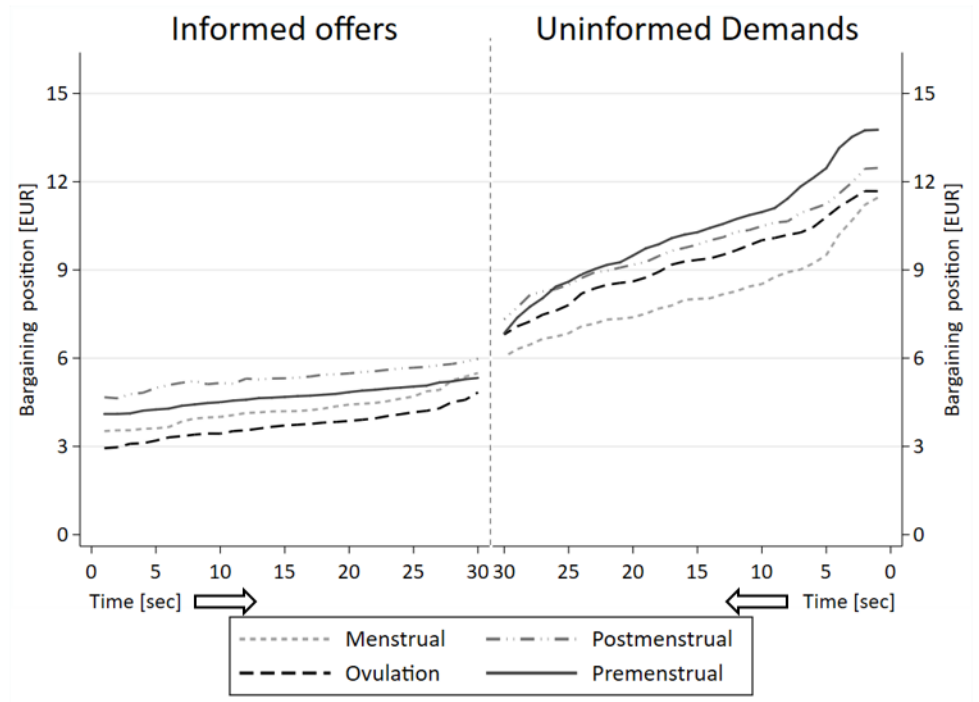

Figure 4.2: Bargaining position across time during the simultaneous bargaining stage

Figure 4.2 also suggests that there are differences in bargaining behavior between informed and uninformed players across the menstrual cycle. For informed players, offers are consistently lower during the ovulation phase versus the other three menstrual phases, and offers during the postmenstrual phase are consistently higher. For uninformed players, average demands seem to be highest during the premenstrual phase and lowest during the menstrual phase. Taking together the different bargaining patterns for both types of players, we conclude that the information setting affects bargaining behavior and potentially the impact of the menstrual cycle on bargaining behavior. Our findings as summarized in Result 1 thus support Hypothesis 1:

Result 1: Bargaining behavior differs between informed and uninformed players.

In the following, we analyze informed and uninformed players' bargaining behavior and payoff consequences separately.

\subsection{Bargaining behavior: positions and concession rates}

To test Hypotheses $2 a$ and $2 b$, we analyze women's bargaining behavior across the menstrual cycle phases separately for informed and uninformed players. Bargaining behavior is captured by the player's willingness to compromise with the other player in a bargaining setting. To measure the level of

\footnotetext{
${ }^{16}$ An additional Pearson's $r$ test also reveals a strong positive correlation between bargaining position and bargaining time for informed players $(r=0.73, p<0.001)$ and uninformed players $(r=-0.93, p<0.001)$.
} 
compromising behavior, we use two variables: we use informed players' initial offers and uninformed players' initial demands to capture players' beliefs and preferences, which they submit before observing the matched partner's bargaining position. Initial average offers $(€ 4.00)$ and demands $(€ 14.00)$ differ substantially. Thus, to reach an agreement, concessions are necessary. To measure compromising behavior in the dynamic interaction with the negotiation partner, we use players' relative concession rates.

We calculate relative concession rates with the relative concession index proposed by Gächter and Riedl (2005). In this index, concessions are normalized by the current bargaining area, which is determined by the difference in the standing bargaining position of the players. For the informed players, the relative concession rate is defined as the difference between the informed player's standing offer and the new offer divided by the current bargaining area. In this case, the bargaining area is the difference between the standing offer of the informed player and the standing demand of the uninformed player. The relative concession rate for the uninformed player is determined analogously. The higher the concession index, the more willingness to concede and compromise does the player reveal. As a robustness check, we also analyze concession rates excluding the initial offer or demands made by the players in Appendix 4.13. We do not find differences with the results obtained with the initial index (see Table 4.13 in Appendix 4.13). ${ }^{17}$

\section{Informed players' bargaining behavior}

Figure 4.3 displays the average initial offers of informed players across the menstrual cycle phases. The data are aggregated over all 10 bargaining rounds. Initial offers vary between 0.00 Euro and 14.30 Euro across the menstrual cycle and are lowest during ovulation (mean value in ovulation: 2.94 Euro, in menstrual: 3.52 Euros; in postmenstrual: 4.67 Euro, and premenstrual: 4.10 Euro). This low level of initial offers during the ovulation phase is consistent with the pattern observed in the left panel of Figure 4.2 where the informed players' average offer is always smaller during ovulation than during the other three phases.

We use regression analysis to test for statistical differences in bargaining behavior between the ovulation phase and the other menstrual phases. The regression estimates are presented in Table 4.2. We use an OLS specification and cluster errors at the matching group level. In all models, we control for Pie size, which is the total amount over which a negotiation pair bargains in the respective round, and Bargaining round. Risk aversion captures an individual's risk aversion coefficient estimated from the risk elicitation task and Social preferences is the informed player's level of benevolence (higher values

\footnotetext{
${ }^{17}$ In Appendix A3, we also explore whether risk and social preferences change across the menstrual cycle for informed and uninformed players. In addition, given the evidence on risk preferences and the menstrual cycle (Buser, 2012, 2011; Lazzaro et al., 2016), in Appendix A3 we also check whether risk preferences are a mediator of the effect of the menstrual cycle on bargaining behavior.
} 


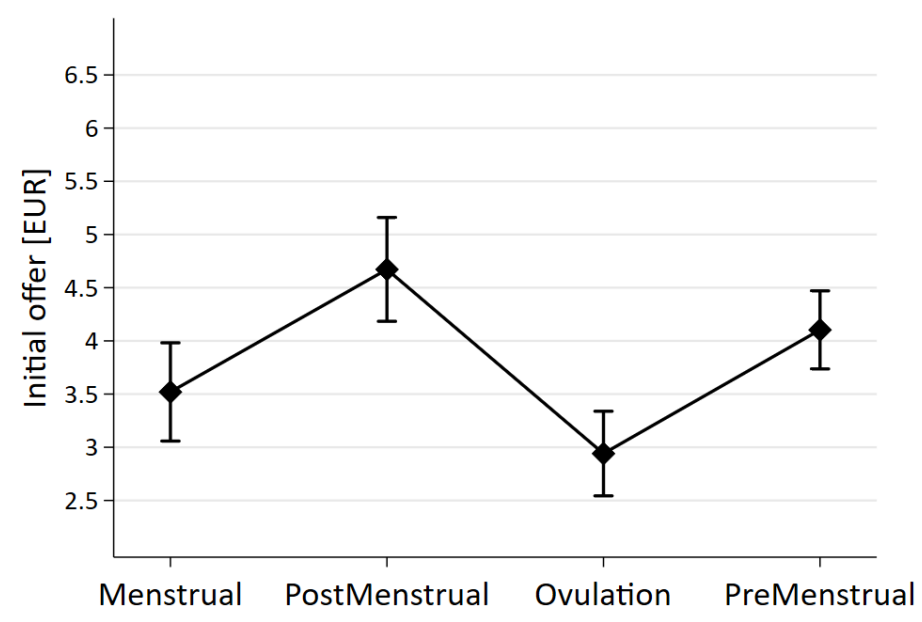

Figure 4.3: Initial offers by informed players.

Note: The figure shows the average offers and corresponding standard errors for each menstrual cycle phase. Standard errors are obtained from OLS regressions clustered on the matching group level and controlling for pie size and bargaining round.

indicate higher levels of risk aversion and benevolence, respectively). We also control for the demographic characteristics of age, nationality, and educational background in all our models.

In models 1 and 2 of Table 4.2, the dependent variable is the informed player's initial offer and the main explanatory variable is the dummy variable Ovulation, which takes the value one if the individual is in the ovulation phase, and zero otherwise. ${ }^{18}$ The reference group of the dummy variable Ovulation is different in models 1 and 2 . In model 1 , the initial offers during ovulation are compared to those in all other menstrual phases. In model 2, the reference group is the premenstrual phase, because the dummy variables Menstruation and Postmenstruation are additionally included in the regression, which takes the value one if the individual is in the respective phase and zero otherwise. To test for differences between the ovulation phase and the menstruation and postmenstruation phases, we run post-estimation Bonferroni-corrected Wald tests, which are reported in the lower part of Table 4.2.

Initial offers are significantly lower during the ovulation phase: Initial offers are on average 1.12 Euro lower during ovulation compared to the rest of the menstrual cycle $(p=0.038$, model 1$)$ and 1.17 Euro lower than during the premenstruation phase $(p=0.020$, model 2$)$. Initial offers are not significantly lower during ovulation than during menstruation $(p=1.000$, model 2), or postmenstruation ( $p=0.167$, model 2). Model 2 of Table 4.2 also shows that there is no significant difference between the premenstrual phase

\footnotetext{
${ }^{18}$ As a robustness check, we adjust the length window of ovulation by reducing the interval by two days and the other menstrual phases accordingly. Our main results do not change with the different definitions of the phases. The robustness check and all the respective tables can be found in Appendix A4.
} 
Table 4.2: Initial offers and relative concession rates for informed players.

\begin{tabular}{|c|c|c|c|c|}
\hline & \multicolumn{2}{|c|}{ Initial offer } & \multicolumn{2}{|c|}{ Concession rate } \\
\hline & (1) & (2) & (3) & (4) \\
\hline Menstruation & & $\begin{array}{l}-0.865 \\
(0.800)\end{array}$ & & $\begin{array}{c}0.029 \\
(0.050)\end{array}$ \\
\hline Postmenstruation & & $\begin{array}{c}0.273 \\
(0.753)\end{array}$ & & $\begin{array}{l}-0.0337 \\
(0.138)\end{array}$ \\
\hline Ovulation & $\begin{array}{c}-1.121^{* *} \\
(0.514)\end{array}$ & $\begin{array}{c}-1.177^{* *} \\
(0.474)\end{array}$ & $\begin{array}{l}-0.034 \\
(0.058)\end{array}$ & $\begin{array}{l}-0.040 \\
(0.047)\end{array}$ \\
\hline Pie size & $\begin{array}{c}0.206^{* * *} \\
(0.022)\end{array}$ & $\begin{array}{c}0.205^{* * *} \\
(0.022)\end{array}$ & $\begin{array}{l}-0.003 \\
(0.007)\end{array}$ & $\begin{array}{l}-0.003 \\
(0.007)\end{array}$ \\
\hline Bargaining round & $\begin{array}{c}-0.119^{* * *} \\
(0.030)\end{array}$ & $\begin{array}{c}-0.119^{* * *} \\
(0.030)\end{array}$ & $\begin{array}{c}0.010 \\
(0.013)\end{array}$ & $\begin{array}{c}0.010 \\
(0.014)\end{array}$ \\
\hline Risk Aversion & $\begin{array}{c}-0.294^{* *} \\
(0.113)\end{array}$ & $\begin{array}{c}-0.305^{* *} \\
(0.119)\end{array}$ & $\begin{array}{c}0.002 \\
(0.020)\end{array}$ & $\begin{array}{c}0.001 \\
(0.020)\end{array}$ \\
\hline Social Preferences & $\begin{array}{c}0.201 \\
(0.130)\end{array}$ & $\begin{array}{c}0.234 \\
(0.145)\end{array}$ & $\begin{array}{c}0.004 \\
(0.024)\end{array}$ & $\begin{array}{c}0.003 \\
(0.027)\end{array}$ \\
\hline Constant & $\begin{array}{c}0.585 \\
(2.484)\end{array}$ & $\begin{array}{l}-0.054 \\
(2.745)\end{array}$ & $\begin{array}{c}0.399 \\
(0.451)\end{array}$ & $\begin{array}{c}0.444 \\
(0.502)\end{array}$ \\
\hline $\begin{array}{l}\text { Observations } \\
\text { R-squared }\end{array}$ & $\begin{array}{c}520 \\
0.348\end{array}$ & $\begin{array}{c}520 \\
0.362\end{array}$ & $\begin{array}{c}475 \\
0.015\end{array}$ & $\begin{array}{c}475 \\
0.016\end{array}$ \\
\hline $\begin{array}{l}\text { Ovulation vs. Menstruation } \\
{[F \text {-test } p \text {-value }]}\end{array}$ & & $\begin{array}{l}-0.312 \\
{[1.000]}\end{array}$ & & $\begin{array}{l}-0.068 \\
{[0.419]}\end{array}$ \\
\hline $\begin{array}{l}\text { Ovulation vs. Postmenstruation } \\
{[F \text {-test } p \text {-value }]}\end{array}$ & & $\begin{array}{c}-1.45 \\
{[0.167]}\end{array}$ & & $\begin{array}{l}-0.005 \\
{[1.000]}\end{array}$ \\
\hline
\end{tabular}

Note: $* p<0.10, * * p<0.05, * * * p<0.01$. Standard errors are clustered at the matching group level (in parentheses). Post-estimation Bonferroni-corrected Wald tests for ovulation versus the menstrual and postmenstrual phases with the effect sizes and the corresponding p-values [in parentheses]. The dependent variable is initial offers in models 1 and 2 and relative concession rates in models 3 and 4. Controls for demographics (age, nationality, and educational background) are included in all four models. The reference group of the dummy variable Ovulation is different in models 1 and 3 and models 2 and 4: While, in models 1 and 3, the initial offers during ovulation are compared to those in all other menstrual phases, the reference group in models 2 and 4 is the premenstrual phase.

and the menstrual or postmenstrual phases $(p=0.277$ and $p=0.720$, model 2 , respectively).

Additional interesting results are obtained from models 1 and 2 in Table 4.2. The pie size affects the initial offer positively as might be expected whereas initial offers go significantly down as the bargaining round increases. With more experience, women seem to become 'tougher' bargainers. Models 1 and 2 also show that initial offers are significantly lower for higher levels of risk aversion, suggesting that informed players with a high degree of risk aversion are more hesitant to make high initial offers to the uninformed players.

To test whether the compromising behavior also differs during the 
interactive bargaining stage, we compare relative concession rates of informed players across menstrual phases. Models 3 and 4 of Table 4.2 have the same specifications as models 1 and 2, respectively, but the dependent variable is now an informed player's average relative concession rate. ${ }^{19}$ The regression analysis suggests that there is no significant variation in the relative concession rates between the ovulation phase (mean value: 0.083 ) and the other menstrual phases (mean value: $0.133, p=0.566$, model 3). Also for the pairwise comparisons, concession rates during ovulation are not significantly different from concession rates in premenstruation $(p=0.906$, model 4$)$, menstruation ( $p=0.419$, model 4$)$, and postmenstruation $(p=1.000$, model 4). The results from models 3 and 4 in Table 4.2 also reveal no significant effects of the pie size ( $p=0.688$ and $p=0.697$, model 3 and 4) and the bargaining round ( $p=0.469$ and $p=0.468$, model 3 and 4) on the informed players concession rates. A similar pattern is observed for risk and social preferences: risk aversion ( $p=0.918$ and $p=0.955$, model 3 and 4) and benevolence ( $p=0.861$ and $p=0.915$, model 3 and 4) do not influence concession rates. We can summarize the findings with respect to Hypothesis 2. $a$ in the following result:

Result 2: Informed players' initial offers are significantly lower during ovulation compared to the other menstrual phases, and in particular, compared to the premenstruation phase. There is no evidence for differences in informed players' relative concession rates during the interactive bargaining stage.

\section{Uninformed players' bargaining behavior}

Figure 4.4 displays the average initial demands of uninformed players across the menstrual cycle (aggregated over the 10 bargaining rounds). Initial demands do not seem to vary substantially across the menstrual cycle.

The regression analysis provided in Table 4.3 tests Hypothesis 2.b, that is, whether initial demands are higher and relative concession rates are lower during the ovulation phase compared to the other phases, and especially, compared to the premenstrual phase. Models 1 and 2 of Table 4.3 display the results for uninformed players' initial demands and models 3 and 4 for their relative concession rates. In all four models presented in Table 4.3, we use an OLS specification and cluster errors at the matching group level and we control for the same explanatory variables used in Table 4.2.

In line with the visual impression in Figure 4.4, the regression results in Table 4.3 confirm that compromising behavior of uninformed players measured by initial demands does not vary significantly across the menstrual cycle. We do not observe significant differences between initial demands made during the ovulation phase and all the other phases pooled $(p=0.303$, model 1$)$. Also the pairwise comparisons of the ovulation phase with premenstruation ( $p=$

\footnotetext{
${ }^{19}$ The reason why there are more observations in models 1 and 2 in comparison to models 3 and 4 is that in the last two models the relative concession rates are calculated for all subjects. Specifically, participants that do not reach a deal and/or do not make a single movement during the entire continuous bargaining stage have no observation for a concession rate.
} 


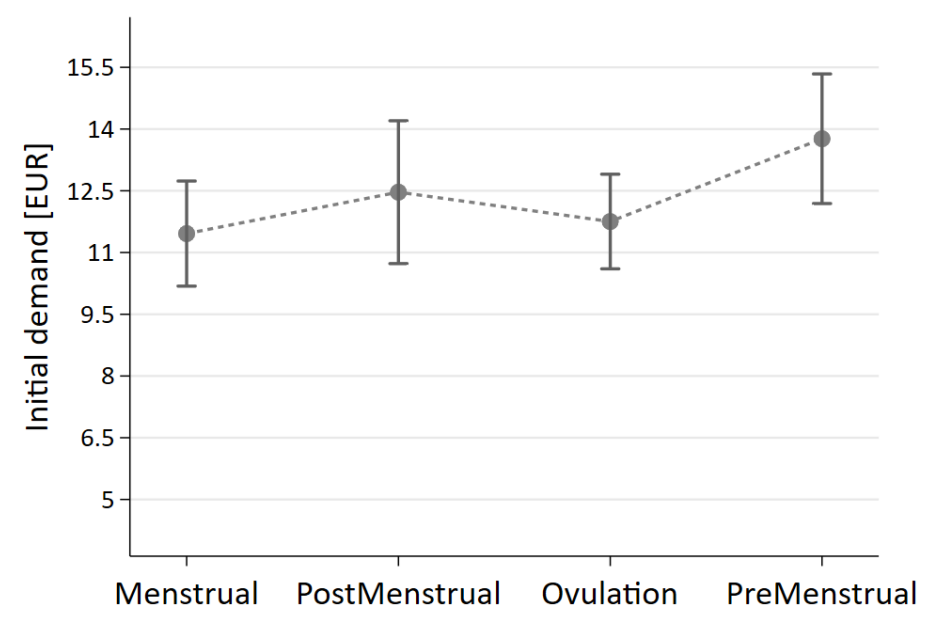

Figure 4.4: Initial demands by uninformed players

Note: The figure shows the average demands and corresponding standard errors for each menstrual cycle phase. Standard errors are obtained from OLS regressions clustered on the matching group level and controlling for pie size and bargaining round.

0.292 , model 2$)$, menstruation ( $p=1.000$, model 2$)$, and postmenstruation ( $p=0.756$, model 2$)$ do not show significant differences.

The remaining results in models 1 and 2 in Table 4.3 reveal that the pie size does not significantly affect initial demands $(p=0.674$ and $p=0.642$, models 1 and 2, respectively). This is expected because uninformed players do not know the pie size while they negotiate (they learn the pie size only afterward). The initial demands of uninformed players increase significantly as the bargaining rounds pass $(p=0.002$ and $p=0.002$, models 1 and 2, respectively). Thus, just like informed players, also uninformed players become less cautious with experience. Risk preferences capture a big part of the variation observed in initial demands. In particular, higher levels of risk aversion significantly increase the level of the initial demands by 0.763 Euros ( $p=0.084$ and $p=0.100$, models 1 and 2). Social preferences, and more precisely benevolence, do not explain initial demands $(p=0.292$ and $p=0.319$, models 1 and 2).

For the relative concession rates, models 3 and 4 in Table 4.3 show that there are no differences in relative concession rates between ovulation and all other menstrual phases pooled ( $p=0.482$, model 3 ) and between the ovulation and the pairwise comparisons with premenstruation $(p=0.517$, model 4), menstruation ( $p=0.335$, model 4), and postmenstruation ( $p=0.956$, model 4$)$. These results partly rejects Hypothesis $2 . b$, given that it was expected to observe lower concession rates during the ovulation phase versus the other menstrual phases. Lastly, the results from 4.3 in Models 3 and 4 reveal the following: the pie size ( $p=0.945$ and $p=0.727$, models 3 and 4 , respectively) and bargaining round ( $p=0.855$ and $p=0.870$, models 3 and 4 , 
Table 4.3: Initial demands and relative concession rates for uninformed players.

\begin{tabular}{|c|c|c|c|c|}
\hline & \multicolumn{2}{|c|}{ Initial demand } & \multicolumn{2}{|c|}{ Concession rate } \\
\hline & (1) & (2) & (3) & (4) \\
\hline Menstruation & & $\begin{array}{l}-1.113 \\
(1.380)\end{array}$ & & $\begin{array}{l}-0.258 \\
(0.223)\end{array}$ \\
\hline Postmenstruation & & $\begin{array}{l}-0.491 \\
(1.550)\end{array}$ & & $\begin{array}{c}0.192 \\
(0.113)\end{array}$ \\
\hline Ovulation & $\begin{array}{l}-1.541 \\
(1.464)\end{array}$ & $\begin{array}{l}-1.856 \\
(1.723)\end{array}$ & $\begin{array}{c}0.100 \\
(0.140)\end{array}$ & $\begin{array}{c}0.091 \\
(0.138)\end{array}$ \\
\hline Pie size & $\begin{array}{l}0.0113 \\
(0.030)\end{array}$ & $\begin{array}{c}0.012 \\
(0.030)\end{array}$ & $\begin{array}{c}0.001 \\
(0.010)\end{array}$ & $\begin{array}{l}0.002 \\
(0.010\end{array}$ \\
\hline Bargaining round & $\begin{array}{c}0.210^{* * *} \\
(0.060)\end{array}$ & $\begin{array}{c}0.210^{* * *} \\
(0.060)\end{array}$ & $\begin{array}{c}0.010 \\
(0.037)\end{array}$ & $\begin{array}{c}0.006 \\
(0.037)\end{array}$ \\
\hline Risk Aversion & $\begin{array}{l}0.762^{*} \\
(0.423)\end{array}$ & $\begin{array}{l}0.712^{*} \\
(0.416)\end{array}$ & $\begin{array}{l}-0.022 \\
(0.025)\end{array}$ & $\begin{array}{l}-0.026 \\
(0.026)\end{array}$ \\
\hline Social Preferences & $\begin{array}{l}-0.606 \\
(0.563)\end{array}$ & $\begin{array}{l}-0.546 \\
(0.537)\end{array}$ & $\begin{array}{c}0.132^{* * *} \\
(0.039)\end{array}$ & $\begin{array}{c}0.153^{* * *} \\
(0.052)\end{array}$ \\
\hline Constant & $\begin{array}{l}8.941^{*} \\
(4.893)\end{array}$ & $\begin{array}{l}8.979^{*} \\
(4.802)\end{array}$ & $\begin{array}{c}0.452 \\
(0.650)\end{array}$ & $\begin{array}{c}0.270 \\
(0.629)\end{array}$ \\
\hline $\begin{array}{l}\text { Observations } \\
\text { R-squared }\end{array}$ & $\begin{array}{c}450 \\
0.150\end{array}$ & $\begin{array}{c}450 \\
0.156\end{array}$ & $\begin{array}{c}413 \\
0.013\end{array}$ & $\begin{array}{c}413 \\
0.020\end{array}$ \\
\hline $\begin{array}{l}\text { Ovulation vs. Menstruation } \\
{[F \text {-test } p \text {-value }]}\end{array}$ & & $\begin{array}{l}-0.743 \\
{[1.000]}\end{array}$ & & $\begin{array}{c}0.167 \\
{[0.335]}\end{array}$ \\
\hline $\begin{array}{l}\text { Ovulation vs. Postmenstruation } \\
{[F \text {-test } p \text {-value }]}\end{array}$ & & $\begin{array}{l}-1.365 \\
{[0.756]}\end{array}$ & & $\begin{array}{c}-0.282 \\
{[0.956]}\end{array}$ \\
\hline
\end{tabular}

Note: $* p<0.10, * * p<0.05, * * * p<0.01$. Standard errors are clustered at the matching group level (in parentheses). Post-estimation Bonferroni-corrected Wald tests for ovulation versus the menstrual and postmenstrual phases with the effect sizes and the corresponding $\mathrm{p}$-values [in parentheses]. The dependent variable is initial offers in models 1 and 2 and relative concession rates in models 3 and 4. Controls for demographics (age, nationality, and educational background) are included in all four models. The reference group of the dummy variable Ovulation is different in models 1 and 3 and models 2 and 4: While, in models 1 and 3, the initial offers during ovulation are compared to those in all other menstrual phases, the reference group in models 2 and 4 is the premenstrual phase.

respectively) do not significantly influence the concession rates. Only social preferences, and more specifically the degree of a participant's benevolence, increase significantly the relative concession rates $(p=0.003$ and $p=0.007$, models 3 and 4, respectively). Summarizing the results with respect to Hypothesis 2.b, we can say the following:

Result 3: Uninformed players' initial demands and relative concession rates do not vary across the menstrual cycle. 


\subsection{Bargaining consequences: Payoffs and deal rates}

Having found evidence for an impact of the menstrual cycle on initial offers of informed players, the question arises whether this translates into negotiation outcomes. We investigate the consequences of bargaining behavior in terms of payoffs and deal rates. Payoffs are a continuous variable that corresponds to the final payoffs obtained at the end of the continuous bargaining stage. Deal rates is a dummy variable at the pair level that takes the value one if a negotiation pair reaches an agreement (in that bargaining round) and the value zero otherwise.

Figure 4.5 displays the average payoffs of the informed and uninformed players unconditional (left panel) and conditional (right panel) on a deal. The data are aggregated over all 10 bargaining rounds. Payoffs vary between - 6 Euro and 22 Euro for informed players, and between 0 Euro and 19.9 Euro for uninformed players. For both types of players, payoffs appear to be higher during the ovulation phase-unconditional and conditional on a deal.

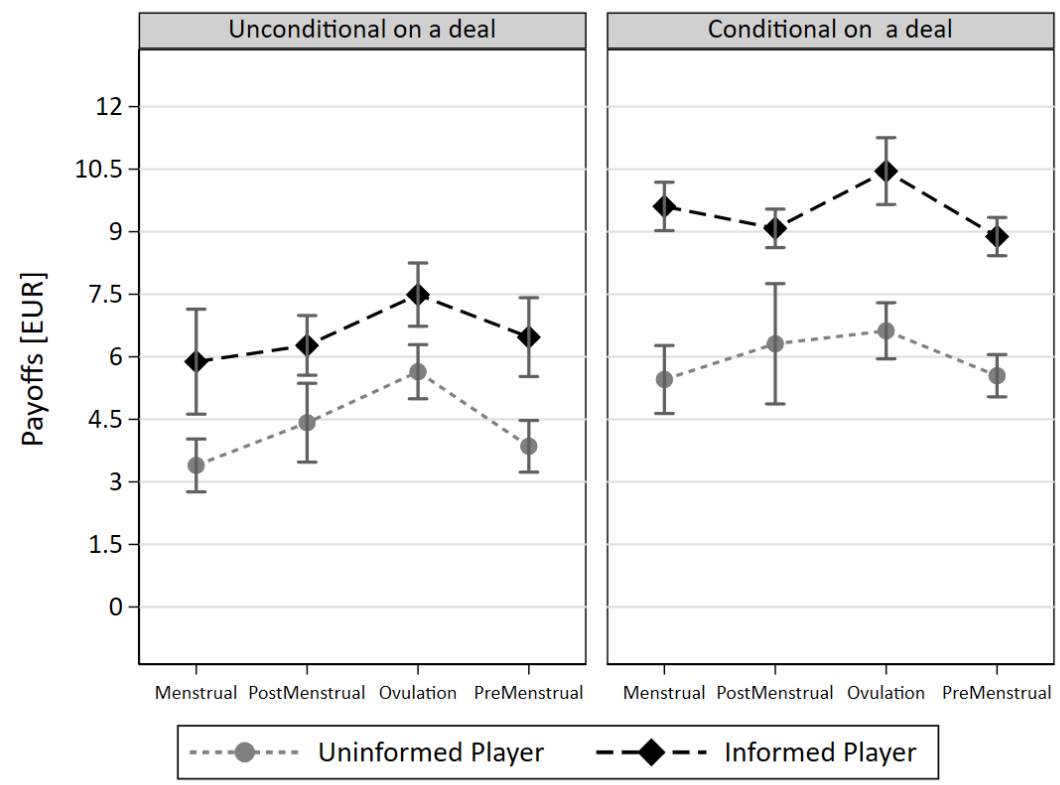

Figure 4.5: Payoffs un/conditional on reaching a deal for informed and uninformed players. Notes: The figure shows the payoffs and corresponding standard errors for each menstrual cycle phase. Standard errors are obtained from OLS regressions clustered on the matching group level and controlling for pie size and bargaining round.

The regression analysis displayed in Table 4.4 tests for differences in payoffs and deal rates of informed players between the ovulation phases and the other three menstrual phases. The dependent variable is unconditional payoffs in models 1 and 2, payoffs conditional on reaching a deal in models 3 and 4 , and deal rates in models 5 and 6 . In all models, we use the same explanatory variables as in Tables 4.2 and 4.3 and add one additional variable: the initial bargaining position of the partner. In a bargaining setting similar to 
ours, Camerer et al. (2019) suggests that the initial position of the partner in a bargaining pair influences strongly the final payoffs of the bargaining round reflecting the interactive nature of the continuous bargaining stage. The partner's initial position serves as a reference point for the paired player (Gächter and Riedl, 2005; Karagözoğlu and Riedl, 2015; Camerer et al., 2019). Lower initial offers of the bargaining partner seem to increase the chances of a deal (Galinsky and Mussweiler, 2001), and this effect is stronger when the initial demand levels are smaller (Camerer et al., 2019).

Table 4.4: Informed players' unconditional payoffs, payoffs conditional on a deal and deal rates.

\begin{tabular}{|c|c|c|c|c|c|c|}
\hline & \multicolumn{2}{|c|}{ Unconditional Payoffs } & \multicolumn{2}{|c|}{ Conditional Payoffs } & \multicolumn{2}{|c|}{ Deal rates } \\
\hline & (1) & (2) & (3) & (4) & (5) & (6) \\
\hline Menstruation & & $\begin{array}{l}-0.045 \\
(0.411)\end{array}$ & & $\begin{array}{c}0.358 \\
(0.536)\end{array}$ & & $\begin{array}{l}-0.075 \\
(0.049)\end{array}$ \\
\hline Postmenstruation & & $\begin{array}{l}-0.115 \\
(0.487)\end{array}$ & & $\begin{array}{l}-0.332 \\
(0.631)\end{array}$ & & $\begin{array}{l}-0.016 \\
(0.048)\end{array}$ \\
\hline Ovulation & $\begin{array}{c}0.768 \\
(0.560)\end{array}$ & $\begin{array}{c}0.728 \\
(0.548)\end{array}$ & $\begin{array}{l}1.163^{* *} \\
(0.501)\end{array}$ & $\begin{array}{l}1.122^{* *} \\
(0.461)\end{array}$ & $\begin{array}{l}-0.003 \\
(0.054)\end{array}$ & $\begin{array}{l}-0.019 \\
(0.060)\end{array}$ \\
\hline Pie size & $\begin{array}{c}0.619^{* * *} \\
(0.029)\end{array}$ & $\begin{array}{c}0.619^{* * *} \\
(0.029)\end{array}$ & $\begin{array}{l}0.715^{* * *} \\
(0.024)\end{array}$ & $\begin{array}{c}0.716^{* * *} \\
(0.023)\end{array}$ & $\begin{array}{c}0.015^{* * *} \\
(0.003)\end{array}$ & $\begin{array}{c}0.015^{* * *} \\
(0.003)\end{array}$ \\
\hline Bargaining round & $\begin{array}{l}0.171^{* *} \\
(0.075)\end{array}$ & $\begin{array}{l}0.171^{* *} \\
(0.075)\end{array}$ & $\begin{array}{c}0.026 \\
(0.052)\end{array}$ & $\begin{array}{c}0.026 \\
(0.051)\end{array}$ & $\begin{array}{l}0.013^{*} \\
(0.007)\end{array}$ & $\begin{array}{l}0.013^{*} \\
(0.007)\end{array}$ \\
\hline Initial Position Partner & $\begin{array}{c}-0.142^{* *} \\
(0.055)\end{array}$ & $\begin{array}{c}-0.142^{* *} \\
(0.056)\end{array}$ & $\begin{array}{l}-0.022 \\
(0.054)\end{array}$ & $\begin{array}{l}-0.027 \\
(0.055)\end{array}$ & $\begin{array}{l}-0.018^{* * *} \\
(0.005)\end{array}$ & $\begin{array}{c}-0.017^{* * *} \\
(0.005)\end{array}$ \\
\hline Risk Aversion & $\begin{array}{c}0.506^{* * *} \\
(0.101)\end{array}$ & $\begin{array}{c}0.498^{* * *} \\
(0.109)\end{array}$ & $\begin{array}{c}0.388^{* * *} \\
(0.104)\end{array}$ & $\begin{array}{c}0.381^{* * *} \\
(0.107)\end{array}$ & $\begin{array}{c}0.028^{* * *} \\
(0.010)\end{array}$ & $\begin{array}{l}0.025^{* *} \\
(0.010)\end{array}$ \\
\hline Social Preferences & $\begin{array}{l}-0.084 \\
(0.104)\end{array}$ & $\begin{array}{l}-0.085 \\
(0.108)\end{array}$ & $\begin{array}{l}-0.293^{* *} \\
(0.128)\end{array}$ & $\begin{array}{c}-0.311^{* *} \\
(0.134)\end{array}$ & $\begin{array}{c}0.002 \\
(0.012)\end{array}$ & $\begin{array}{c}0.004 \\
(0.011)\end{array}$ \\
\hline Constant & $\begin{array}{l}-0.568 \\
(1.230)\end{array}$ & $\begin{array}{l}-0.472 \\
(1.354)\end{array}$ & $\begin{array}{l}-0.398 \\
(1.860)\end{array}$ & $\begin{array}{c}0.048 \\
(1.958)\end{array}$ & $\begin{array}{l}0.532^{* *} \\
(0.198)\end{array}$ & $\begin{array}{l}0.511^{* *} \\
(0.195)\end{array}$ \\
\hline $\begin{array}{l}\text { Observations } \\
\text { R-squared }\end{array}$ & $\begin{array}{c}520 \\
0.518\end{array}$ & $\begin{array}{c}520 \\
0.518\end{array}$ & $\begin{array}{c}364 \\
0.821\end{array}$ & $\begin{array}{c}364 \\
0.822\end{array}$ & $\begin{array}{c}520 \\
0.0995\end{array}$ & $\begin{array}{c}520 \\
0.102\end{array}$ \\
\hline $\begin{array}{l}\text { Ovulation vs. Menstr. } \\
{[F \text {-test } p \text {-value }]}\end{array}$ & & $\begin{array}{c}0.778 \\
{[0.490]}\end{array}$ & & $\begin{array}{c}0.764 \\
{[0.521]}\end{array}$ & & $\begin{array}{c}0.06 \\
{[0.776]}\end{array}$ \\
\hline $\begin{array}{l}\text { Ovulation vs. Postmentr. } \\
{[F \text {-test } p \text {-value }]}\end{array}$ & & $\begin{array}{c}0.843 \\
{[0.500]}\end{array}$ & & $\begin{array}{c}1.454 \\
{[0.135]}\end{array}$ & & $\begin{array}{c}0.00 \\
{[1.000]}\end{array}$ \\
\hline
\end{tabular}

Note: $* p<0.10, * * p<0.05, * * * p<0.01$. Standard errors are clustered at the matching group level (in parentheses). Post-estimation Bonferroni-corrected Wald tests for ovulation versus the menstrual and postmenstrual phases with the effect size and the corresponding p-values [in parentheses]. The dependent variable is unconditional payoffs in models 1 and 2, payoffs conditional on a deal in models 3 and 4, and deal rates in models 5 and 6. Controls for demographics (age, nationality, and educational background) are included in all six models. The reference group of the dummy variable Ovulation is different in models 1, 3 and 5 and models 2, 4 and 6: While, in models 1, 3 and 5 the initial offers during ovulation are compared to those in all other menstrual phases, the reference group in models 2,4 and 6 is the premenstrual phase. 
Models 1 and 2 of Table 4.4 show that informed players' payoffs unconditional on a deal do not vary across the menstrual cycle. However, the results for payoffs conditional on reaching a deal in models 3 and 4 suggest that informed players' payoffs are on average 1.163 Euros higher during the ovulation phase compared to the rest of the menstrual phases $(p=0.028$, model 3) and 1.122 Euros higher than during the premenstrual phase $(p=0.022$, model 4$)$. This result is interesting because it suggests that the decrease in compromising behavior observed during the ovulation phase translates into higher payoffs for the informed players once it is controlled for a deal.

The results from the pairwise comparisons in Table 4.4 show that informed players' unconditional payoffs do not significantly differ between ovulation and menstruation ( $p=0.490$, model 2 ) or postmenstruation ( $p=0.500$, model 2). Similarly, conditional payoffs during ovulation are not significantly different from those during the menstruation ( $p=0.0 .764$, model 4$)$, or postmenstruation ( $p=0.135$, model 4$)$. In addition, there is no significant difference between the premenstrual phase and the menstrual or postmenstrual phases, for models 1, 2, 3 and 4 in Table 4.4.

Additional interesting results are obtained from Table 4.4 for informed player's payoffs. Unconditional payoffs significantly increase with a bigger pie size ( $p<0.001$, models 1 and 2$)$ and with more experience of the players ( $p=0.031$, models 1 and 2), and decrease the higher is the initial demand of the uninformed matched partner ( $p=0.017$, models 1 and 2). Conditional payoffs are also positively influenced by the pie size $(p<0.001$, models 1 and 2 ), but not by the bargaining round ( $p=0.615$, models 1 and 2) or by the initial demand of the partner ( $p=0.632$, models 1 and 2). Risk preferences influence conditional and unconditional payoffs as well. Models 1, 2, 3, and 4 suggest that the more risk averse an informed player is, the higher is the payoff obtained. In addition, for conditional payoffs, we observe a decrease when the players are more benevolent.

The divergence in results for informed players' unconditional and conditional payoffs during ovulation is explained by the different profile of risk preferences between the informed players that reach a deal and the ones that do not reach a deal during the ovulation phase. Specifically, we observe that risk aversion levels increase payoffs (Table 4.4, models 1 to 4) and deal rates (Table 4.4, models 5 and 6). This suggests that risk averse informed players are better at reaching a deal and ensuring higher payoffs for themselves. When looking at conditional payoffs, we focus only on people who reach a deal and have high-risk aversion levels. In addition, for the average risk aversion levels of women in the ovulation phase, we see that the players in the ovulation phase who reach a deal are more risk averse (mean risk coefficient: 0.263 ) in comparison to the players in the ovulation phase who do not reach a deal (mean risk coefficient: 0.026). In consequence, in models 3 and 4 of Table 4.4 there is a selection of high-risk averse informed players during the ovulation phase, which increases the payoffs only during 
ovulation once we account for the cases when there was a deal. ${ }^{20}$

The last two models of Table 4.4 display the results for the informed players' deal rates. Models 5 and 6 in Table 4.4 suggest that there are no differences in deal rates between ovulation and all other menstrual phases pooled ( $p=0.960$, model 5 ) and between the ovulation and the pairwise comparisons with premenstruation ( $p=0.757$, model 6), menstruation ( $p=0.776$, model 6$)$, and postmenstruation ( $p=1.00$, model 6). We summarize the results obtained for informed players' payoffs and deal rates in the following result:

Result 4: Informed players' payoffs are significantly higher during ovulation than during the rest of the menstrual cycle and in particular compared to the pre-menstrual phase when a deal was reached. There is no evidence for differences in informed players' deal rates across the menstrual cycle.

To test for differences in payoffs and deal rates of uninformed players between the ovulation phases and the other three menstrual phases, we use the regression analysis displayed in Table 4.5. Similar to the analysis for informed players, the dependent variable in Table 4.5 is unconditional payoffs in models 1 and 2, payoffs conditional on reaching a deal in models 3 and 4, and deal rates for models 5 and 6 . The explanatory variables are the same as those used in Table 4.4.

Models 1 and 2 of Table 4.5 reveal that uninformed players' unconditional payoffs are on average 1.05 Euros higher during the ovulation phase compared to the rest of the menstrual phases $(p=0.030$, model 1$)$ and 0.945 Euros higher than during the premenstrual phase $(p=0.045$, model 2$)$. In contrast, once payoffs are conditional on a deal, we do not observe differences in uninformed players' payoffs during the ovulation phase and the other three phases ( $p=$ 0.891 , model 3 ), and the premenstrual phase ( $p=0.930$, model 4$)$.

Additional results are obtained from Table 4.5 for the uninformed players' payoffs. Similar to the payoffs for informed players, the pie size, bargaining round and initial position of the partner are positively associated with uninformed players' payoffs. Table 4.5 also suggests that uninformed players' risk and social preferences do not influence unconditional $(p=0.846$ and $p=0.156$, model 1 ; and $p=0.645$ and $p=0.143$, model 2) and conditional payoffs $(p=0.483$ and $p=0.143$, model 3 ; and $p=0.462$ and $p=0.175$, model 4).

The last two models of Table 4.5 show that uninformed players' deal rates are higher during ovulation than during the other phases $(p=0.034$, model 5) and than during the premenstrual phase $(p=0.048$, model 6$)$. This high frequency of deal rates during ovulation helps to explain the differences in results between unconditional and conditional payoffs. Specifically, most of the uninformed players in ovulation make a deal (an average of $85 \%$ of them as shown in Table 4.6). Since models 1 and 2 of Table 4.5 include uninformed

\footnotetext{
${ }^{20}$ We acknowledge that this is a speculative proposition, and further analysis is required to confidently make this claim.
} 
Table 4.5: Uninformed players' unconditional payoffs, payoffs conditional on a deal and deal rates.

\begin{tabular}{|c|c|c|c|c|c|c|}
\hline & \multicolumn{2}{|c|}{ Unconditional Payoffs } & \multicolumn{2}{|c|}{ Conditional Payoffs } & \multicolumn{2}{|c|}{ Deal rates } \\
\hline & (1) & (2) & (3) & (4) & (5) & (6) \\
\hline Menstrual & & $\begin{array}{l}-0.801^{* *} \\
(0.364)\end{array}$ & & $\begin{array}{l}-0.233 \\
(0.554)\end{array}$ & & $\begin{array}{l}-0.077 \\
(0.081)\end{array}$ \\
\hline Post-Menstrual & & $\begin{array}{c}0.218 \\
(0.335)\end{array}$ & & $\begin{array}{c}0.120 \\
(0.404)\end{array}$ & & $\begin{array}{l}-0.001 \\
(0.072)\end{array}$ \\
\hline Ovulation & $\begin{array}{l}1.055^{* *} \\
(0.458)\end{array}$ & $\begin{array}{l}0.945^{* *} \\
(0.447)\end{array}$ & $\begin{array}{c}0.038 \\
(0.274)\end{array}$ & $\begin{array}{c}0.028 \\
(0.317)\end{array}$ & $\begin{array}{l}0.150^{* *} \\
(0.067)\end{array}$ & $\begin{array}{l}0.135^{* *} \\
(0.065)\end{array}$ \\
\hline Pie size & $\begin{array}{l}0.119^{* * *} \\
(0.024)\end{array}$ & $\begin{array}{c}0.123^{* * *} \\
(0.025)\end{array}$ & $\begin{array}{c}0.132^{* * *} \\
(0.021)\end{array}$ & $\begin{array}{c}0.134^{* * *} \\
(0.021)\end{array}$ & $\begin{array}{l}0.007^{*} \\
(0.004)\end{array}$ & $\begin{array}{l}0.008^{*} \\
(0.004)\end{array}$ \\
\hline Bargaining round & $\begin{array}{l}0.112^{* *} \\
(0.049)\end{array}$ & $\begin{array}{l}0.111^{* *} \\
(0.049)\end{array}$ & $\begin{array}{l}0.070^{* *} \\
(0.028)\end{array}$ & $\begin{array}{l}0.070^{* *} \\
(0.027)\end{array}$ & $\begin{array}{c}0.012 \\
(0.008)\end{array}$ & $\begin{array}{c}0.012 \\
(0.008)\end{array}$ \\
\hline Initial Position Partner & $\begin{array}{l}0.702^{* * *} \\
(0.079)\end{array}$ & $\begin{array}{c}0.698^{* * *} \\
(0.080)\end{array}$ & $\begin{array}{c}0.731^{* * *} \\
(0.056)\end{array}$ & $\begin{array}{c}0.728^{* * *} \\
(0.059)\end{array}$ & $\begin{array}{l}0.018^{* *} \\
(0.008)\end{array}$ & $\begin{array}{l}0.018^{* *} \\
(0.008)\end{array}$ \\
\hline Risk Aversion & $\begin{array}{l}-0.014 \\
(0.069)\end{array}$ & $\begin{array}{l}-0.037 \\
(0.080)\end{array}$ & $\begin{array}{l}-0.082 \\
(0.115)\end{array}$ & $\begin{array}{l}-0.087 \\
(0.117)\end{array}$ & $\begin{array}{c}0.006 \\
(0.009)\end{array}$ & $\begin{array}{c}0.003 \\
(0.010)\end{array}$ \\
\hline Social Preferences & $\begin{array}{l}-0.203 \\
(0.139)\end{array}$ & $\begin{array}{l}-0.153 \\
(0.101)\end{array}$ & $\begin{array}{l}-0.261 \\
(0.172)\end{array}$ & $\begin{array}{l}-0.247 \\
(0.177)\end{array}$ & $\begin{array}{c}0.001 \\
(0.022)\end{array}$ & $\begin{array}{c}0.006 \\
(0.020)\end{array}$ \\
\hline Constant & $\begin{array}{c}0.527 \\
(1.391)\end{array}$ & $\begin{array}{c}0.255 \\
(1.335)\end{array}$ & $\begin{array}{c}0.632 \\
(1.232)\end{array}$ & $\begin{array}{c}0.519 \\
(1.198)\end{array}$ & $\begin{array}{c}0.771^{* * *} \\
(0.189)\end{array}$ & $\begin{array}{c}0.756^{* * *} \\
(0.195)\end{array}$ \\
\hline $\begin{array}{l}\text { Observations } \\
\text { R-squared }\end{array}$ & $\begin{array}{c}450 \\
0.485\end{array}$ & $\begin{array}{c}450 \\
0.492\end{array}$ & $\begin{array}{c}319 \\
0.738\end{array}$ & $\begin{array}{c}319 \\
0.739\end{array}$ & $\begin{array}{c}450 \\
0.077\end{array}$ & $\begin{array}{c}450 \\
0.080\end{array}$ \\
\hline $\begin{array}{l}\text { Ovulation vs. Menstr. } \\
{[F \text {-test } p \text {-value }]}\end{array}$ & & $\begin{array}{c}1.746 \\
{[0.007]}\end{array}$ & & $\begin{array}{c}0.261 \\
{[0.137]}\end{array}$ & & $\begin{array}{c}0.212 \\
{[1.000]}\end{array}$ \\
\hline $\begin{array}{l}\text { Ovulation vs. Postmentr. } \\
{[F \text {-test } p \text {-value }]}\end{array}$ & & $\begin{array}{c}0.727 \\
{[0.410]}\end{array}$ & & $\begin{array}{c}0.092 \\
{[1.000]}\end{array}$ & & $\begin{array}{c}0.136 \\
{[1.000]}\end{array}$ \\
\hline
\end{tabular}

Note: $* p<0.10, * * p<0.05, * * * p<0.01$. Standard errors are clustered at the matching group level (in parentheses). Post-estimation Bonferroni-corrected Wald tests for ovulation versus the menstrual and postmenstrual phases with the effect size and the corresponding p-values [in parentheses]. The dependent variable is unconditional payoffs in models 1 and 2, payoffs conditional on a deal in models 3 and 4, and deal rates in models 5 and 6. Controls for demographics (age, nationality, and educational background) are included in all six models. The reference group of the dummy variable Ovulation is different in models 1, 3 and 5 and models 2, 4 and 6: While, in models 1,3 and 5 the initial offers during ovulation are compared to those in all other menstrual phases, the reference group in models 2, 4 and 6 is the premenstrual phase.

players that do not make a deal, and that in consequence have a payoff of zero, the differences in unconditional payoffs between ovulation and the other menstrual phases are high in these two models. In contrast, when in models 3 and 4 of Table 4.5 we restrict to the uninformed players that a reach deal, the significant difference observed during ovulation disappears. We summarize the results obtained for uninformed players' payoff and deal rates in the following result: 
Result 5: Uninformed players' unconditional payoffs and deal rates are higher during ovulation. Conditional on having reached a deal, uninformed players' payoffs do not vary across the menstrual cycle phases.

To complement the results in Tables 4.4 and 4.5 for deal rates, we look at the average deal rates across the menstrual phases for informed and uninformed players in Table 4.6. On average, informed players have the highest deal rates when they are in the premenstrual phase and the lowest deal rates when they are in the menstruation phase. For the uninformed players, the highest deal rate is achieved during the ovulation phase and the lowest during the menstruation phase.

Table 4.6: Average deals rates for informed and uninformed players

\begin{tabular}{|c|c|c|c|c|}
\hline & \multicolumn{4}{|c|}{ Player's phase } \\
\hline & Menstruation & Postmens. & Ovulation & Premens. \\
\hline $\begin{array}{l}\text { Informed player's deal rate } \\
{[n=520]}\end{array}$ & $\begin{array}{c}61.25 \% \\
{[80]}\end{array}$ & $\begin{array}{c}69.09 \% \\
{[110]}\end{array}$ & $\begin{array}{c}71.66 \% \\
{[120]}\end{array}$ & $\begin{array}{c}72.85 \% \\
{[210]}\end{array}$ \\
\hline $\begin{array}{l}\text { Uninformed player's deal rate } \\
{[\mathrm{n}=450]}\end{array}$ & $\begin{array}{c}62.23 \% \\
{[90]}\end{array}$ & $\begin{array}{l}70 \% \\
{[80]}\end{array}$ & $\begin{array}{l}85 \% \\
{[80]}\end{array}$ & $\begin{array}{l}68.5 \% \\
{[200]}\end{array}$ \\
\hline
\end{tabular}

Note: Average percentage of deal rates by phase for informed and uninformed player. Corresponding number of pairs in the specific row player's menstrual phase in [parentheses].

We summarize the results for deal rates for informed and uninformed players in the following result:

Result 6: Deal rates are particularly high for pairs of players where the informed player is in the premenstruation and the uninformed player is in the ovulation phase. In contrast, deal rates are particularly low for pairs where the informed and uninformed player is in the menstruation phase.

\section{Discussion and conclusions}

In a controlled laboratory experiment, we provide evidence of variation in bargaining behavior across the menstrual cycle. Using an unstructured bilateral bargaining setting proposed by Camerer et al. (2019), characterized by an asymmetry of information between the two involved parts, we show that the effect of the menstrual cycle on bargaining behavior and outcomes depends on the information setting. We analyze bargaining behavior separately for informed players and uninformed players.

Based on existing literature, we hypothesize that women are less willing to compromise during the ovulation phase compared to the other phases, and especially, compared to the premenstrual phase. In the separate analysis of informed and uninformed players, our results confirm our hypotheses mainly for only the players who are not affected by the asymmetry of information, i.e., informed players. In particular, we observe that informed players' 
bargaining behavior is less compromising during the ovulation phase compared to the other three menstrual phases, and especially to the premenstruation phase. We measure compromising behavior with the magnitude of the initial offers made by the informed players in the bargaining context. This result is consistent with the evolutionary argument of women engaging in less competitive behavior during their infertile phase and pregnancy (i.e., premenstruation phase) than in their fertile phase (i.e., ovulation phase), which is when women compete for the fittest males for reproduction purposes (Lazzaro et al., 2016). In our bargaining environment, a competitive behavior means less willingness to compromise or lower initial offers to the other player. Interestingly, we also observe that the changes in bargaining behavior for informed players are reflected in the conditional payoffs they earn in bargaining in these two menstrual phases. Specifically, we observe that being less willing to compromise during the ovulation phase versus the premenstruation phase pays off in terms of final payoffs conditional on reaching a deal during the ovulation phase.

For informed players, we do not find variation across the menstrual cycle in our third measurement of concession behavior, which is relative concession rates. This null effect is not surprising given the patterns observed in Figure 4.2 for the initial and final offers (i.e., the last offer made in the second 30 during the simultaneous bargaining stage). The impact of the menstrual cycle on initial and final offers seems to be of similar magnitude, and in consequence, there is no effect of the menstrual cycle on concession rates. Unfortunately, to make confidently this claim a more detailed analysis will be required in terms of informed players' final and initial offers.

Our results for the informed player also reflect another interesting pattern; the variation in bargaining behavior and consequences is only significant when we compare the ovulation phase with the other three phases together and the premenstrual phase alone, but not between the other phases. This pattern fits with the findings from the medical literature that suggest that behavioral changes over the menstrual cycle are more salient when the two sex hormones are facing drastic changes in their levels (i.e., in the transition from the ovulation phase to the premenstruation phase) (Lazzaro et al., 2016).

It is worth mentioning that for informed players, even though risk aversion levels play a role in bargaining behavior, the variation we observe in initial offers during the menstrual cycle is independent of this trait. To look at the effect of the menstrual cycle mediated by risk preferences, we perform an additional analysis where we interact risk preferences with each of the four menstrual phases. The results suggest that although risk preferences help to explain some of the differences in bargaining behavior for informed players, the effect of risk aversion on bargaining outcomes is the same across all the menstrual phases. The results from this analysis can be found in Appendix A3.

Our analysis for uninformed players provides evidence of a null effect of the menstrual cycle on bargaining behavior for the players that are affected by the asymmetry of information. Specifically, contrary to our hypothesis we observe that compromising behavior, measured by the level of initial demands and relative concession rates, does not change across the menstrual cycle for 
uninformed players. Interestingly, risk preferences seem to play a substantial role in the uninformed players' bargaining behavior. The interpretation we give to these findings is that the lack of information for uninformed players gives room for risk aversion to playing a significant role in their bargaining behavior. Furthermore, the uncertainty present in the environment for these players seems to be big enough to cancel out any potential effect of the menstrual cycle phases on their bargaining behavior.

For uninformed players' bargaining behavior, we also look at the variation in payoffs and deal rates across the menstrual cycle. We observe that uninformed players obtain higher unconditional payoffs (i.e., payoffs including disagreements payoffs) and have higher deal rates during ovulation in comparison to the other menstrual phases, and especially versus premenstruation. Similar to the findings for informed players' bargaining behavior during ovulation, the result for uninformed players bargaining consequences is in line with the evidence that suggests that women are more competitive during ovulation (Lazzaro et al., 2016; Buser, 2012), and therefore, secure better bargaining outcomes in terms of payoffs and agreement rates in this menstrual phase.

The results for deal rates at the pair level suggest that the highest rate occurs when the informed player is in the premenstruation phase and the uninformed is in the ovulation phase. This is consistent with our previous findings of higher initial offers made by informed players during premenstruation together with the increase in efficacy of uninformed players to reach a deal during the ovulation phase. In contrast, the lowest percentage of deal rates happens when both players in a pair are in the menstruation phase (61\% of pairs reach a deal). Although we expected to have higher deal rates during the premenstruation phase in comparison to the ovulation phase, we do not have any specific reason for deal rates to be lower during the menstruation phase when compared to the premenstruation phase. Perhaps this finding is related to the fact that informed players are less risk averse during the menstruation phase when compared to the premenstrual phase (see Appendix A3). The finding suggests that having a risk-loving player in a pair can decrease the chances of reaching an agreement.

The hormonal levels of non-contraceptive takers differ from the levels of the ones taking hormonal contraceptives (Golobof and Kiley, 2016). For this reason, we do not assign contraceptive takers to the four menstrual phases and analyze them separately. In Appendix A5, we report the results on contraceptive takers' bargaining behavior. Our findings for contraceptive takers suggest that there are no differences in bargaining outcomes and consequences between the intake and the break period. Our analysis for contraceptive takers is consistent with the recent findings from Ranehill et al. (2018), which suggest that hormonal changes induced by oral contraceptives do not affect economic behavior.

Because of the purely female sample, we can only draw conclusions on women's bargaining behavior when paired with other women. Bargaining behavior towards men is equally relevant and could potentially differ. For instance, during the ovulation phase, women are more attracted towards men 
(Penton-Voak and Perrett, 2000). In contrast, during the premenstrual phase, they are less attracted to them (Jones et al., 2005). This could potentially affect bargaining behavior and remains an open question of how women bargain differently across the menstrual cycle in the presence of men. Since women revealed highly sensitive and intimate information, we decided against a mixed-sex sample to create a safe and comfortable environment for the participating women. However, a natural future of research is to analyze bargaining behavior across the menstrual cycle in a mixed-gender environment.

When looking isolated at variation in risk and social preferences across the menstrual cycle, we observe a significant change in risk aversion levels but not in social preferences (for details of this analysis please refer to Appendix A3). Specifically, we observe that informed players are more risk averse during the premenstruation phase when compared to the menstruation and postmenstruation phases. It was expected to have higher risk aversion levels during the premenstruation phase in comparison to the ovulation phase (Lazzaro et al., 2016), but we did not have any specific hypotheses on the menstruation and postmenstruation phases. We believe this result needs to be interpreted carefully and requires further exploration, given that in our study it was not the main focus to study risk preferences itself. Also, most of the literature on risky behavior and the menstrual cycle has suggested no variation of risk aversion across the menstrual phases (Buser, 2012; Ranehill et al., 2018; Schipper, 2014). For altruistic behavior, similar to Ranehill et al. (2018) we observe no variation of this type of behavior across the menstrual cycle. However, as mentioned earlier for risk preferences, social preferences were also not the main focus in our study and we are therefore hesitant to derive any main conclusions about it.

Our results contribute to the growing literature on the impact of hormones on economic behavior, such as testosterone and estrogen. Although early evidence suggested a potential effect of these hormones on economic behavior (Kosfeld et al., 2005; Burnham, 2007; Apicella et al., 2008), studies with a bigger sample size have found a null effect of hormones on a range of economic behaviors ( $n=200$ in Zethraeus et al. (2009); $n=340$ in (Ranehill et al., 2018)). These different findings speak to the need for large-scale studies to detect false positives in the context of hormones and economic behavior. We believe that with our sample size of 166 subjects we account partially for this potential problem. Also, our findings support the null effect of hormones on economic behavior, and particularly on bargaining behavior, for players whose environment is very uncertain. We think that this finding can open a discussion on the role of hormones conditional on the level of uncertainty present in the environment. For instance, for the specific case of competition, it is well known that risk preferences are a crucial determinant of competitive behavior (Niederle and Vesterlund, 2007; van Veldhuizen, 2017; Gillen et al., 2019). Therefore, a null effect of hormones on competitive behavior as the one suggested by Ranehill et al. (2018) can be due to the presence of uncertainty in the competitive environment, and not due to the null effect of hormones on competitive behavior itself. For instance, in our bargaining 
context, we see that bargaining behavior varies across the menstrual cycle only for players that are not affected by uncertainty (i.e., the ones fully informed). 


\section{Appendix}

\section{A1. Experimental design details}

In this subsection, we provide detail information of the elicitation method used to capture risk and social preferences.

\section{Part 3: Social preferences}

For eliciting social preferences, we use the equality-equivalence test developed by Kerschbamer (2015). In this test, subjects make incentivize individual binary choices between ten allocations that have an own payoff for the decision maker and a payoff for a random matched player, named the passive player. As displayed by the two first columns and two last columns in Table 4.7, there are two types of allocations. The ones labeled as Right represent symmetric allocations, where both players receive the same amount (i.e., $2 €$ for each player). The ones labeled as Left represent asymmetric allocations, where both players receive different amounts. Specifically, for the Left allocations there are two blocks of 5 allocations each: a disadvantageous inequality block and an advantageous inequality block. In the former block, the decision maker always gets a smaller amount than the passive player, whereas in the latter block the opposite happens.

Table 4.7: Choices for social preferences

\begin{tabular}{lclll}
\hline Left & & & Right \\
\cline { 1 - 2 } \cline { 1 - 1 } You get & Passive person gets & & You get & Passive person gets \\
\cline { 1 - 2 } Disadvantegeous inequality block & & \\
$1.60 €$ & $2.60 €$ & & $2.00 €$ & $2.00 €$ \\
$1.80 €$ & $2.60 €$ & & $2.00 €$ & $2.00 €$ \\
$2.00 €$ & $2.60 €$ & & $2.00 €$ & $2.00 €$ \\
$2.20 €$ & $2.60 €$ & & $2.00 €$ & $2.00 €$ \\
$2.40 €$ & $2.60 €$ & & $2.00 €$ & $2.00 €$ \\
Advantageous inequality block & & \\
$1.60 €$ & $1.40 €$ & & $2.00 €$ & $2.00 €$ \\
$1.80 €$ & $1.40 €$ & & $2.00 €$ & $2.00 €$ \\
$2.00 €$ & $1.40 €$ & & $2.00 €$ & $2.00 €$ \\
$2.20 €$ & $1.40 €$ & & $2.00 €$ & $2.00 €$ \\
$2.40 €$ & $1.40 €$ & $2.00 €$ & $2.00 €$ \\
\hline
\end{tabular}

Note: this table was not displayed to the subjects. There were presented each row in separate screens and in a random order.

We created an index that measures the degree of benevolent (or malevolent) behavior given the choices subjects make in each of the two blocks. ${ }^{21}$ Specifically, we categorized a player with a high degree of

\footnotetext{
${ }^{21}$ We do not use the measurement of distributional preferences suggested by Kerschbamer (2015) because the switching behavior of our subjects doesn't allow for a rationalization of such choices. We have more that one switching per block for some subjects. This inconsistent
} 
benevolence if when confronted with the binary choices in the disadvantageous inequality block, he or she chooses most of the time the asymmetric allocation; and when confronted with the binary choices in the advantageous inequality block, he or she chooses most of the time the symmetric allocation.

Following the design of Kerschbamer (2015), we used a double role assignment protocol. That is, each subject gets two payoffs in this task, one obtained for the role of decision maker and the other one for the role of passive player. At the end of the experiment, the earnings from this task correspond to the payoffs from one of the ten choices made as a active decision maker that is randomly selected with equal probability plus the payoffs obtained as a passive player.

\section{Tracking of the menstrual cycle and estimation of the phases details}

Three months before the lab sessions take place, participants attend an introductory meeting where we inform them about the duration of the whole experiment and also provide them detailed instructions on how to track their menstrual cycle. ${ }^{22}$ To track the menstrual cycle, participants have an electronic menstrual cycle calendar as the one displayed in Figure 4.6. In the electronic calendar, participants fill in during three months the starting day and the duration of their menstruation. Additionally, women using a hormonal contraceptive method provide information about their contraceptive method and the pill intake period if applicable. Participants receive reminders every two weeks to ensure they fill out the calendar monthly.

Figure 4.6: Electronic menstrual cycle calendar

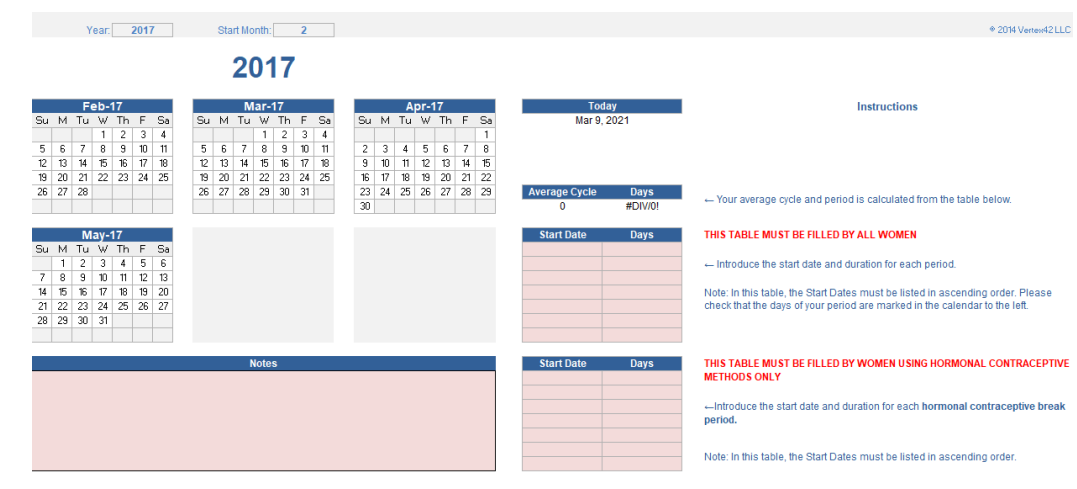

About two weeks before the experimental sessions with the bargaining game, participants hand in the menstrual cycle calendar to the researchers and are assigned to one of the scheduled experimental sessions. ${ }^{23}$ Additionally, to

\footnotetext{
switching behavior makes difficult to estimate subjects' distributional preferences.

${ }^{22}$ Participants are never told what the lab sessions are about, they only know they will have to attend to one session of a behavioral experiment after the three months tracking period.

${ }^{23}$ Importantly, to ensure a careful treatment of the menstrual cycle information, participants always meet the same female researcher over the whole duration of the experiment.
} 
ensure anonymity of the data provided participants are assigned an individual ID. We allocate participants to the experimental sessions in advance to ensure having a balance sample in terms of women from different menstrual phases. Hence, after two months and a half of tracking the menstrual cycle, we could estimate approximately the phase in which our participants are the day of the behavioral experiment session. Lastly, during the experimental session, participants hand in their menstrual cycle calendar for the last time.

As displayed in Figure 4.1, we take an average cycle of 28 days, the individual average menstruation length, a length of 5 days for the ovulation phase and 12 days for the premenstrual phase to estimate the menstrual phases in the following fours steps:

1. The menstrual phase is obtained by using the last day of their cycle reported in the calendar and their individual average menstruation duration.

2. The premenstrual phase is calculated by using the expected next menstruation date and average duration of 12 days for this phase.

3. We calculated the ovulation phase by using the expected next menstruation date and an average duration of 5 days for this phase.

4. Lastly, the Postmenstrual phase is obtained by using the day after the menstrual phase and the date right before the ovulation phase. ${ }^{24}$ Importantly, the day of the experiment session we asked subjects whether they were experiencing menstrual bleeding on that day, this helps us to allocate them correctly between the menstrual and Postmenstrual phases.

For most participants, we use a backwards estimation of their menstrual phase. That is, if participants expect their next menstruation to start after the experimental session, we estimate their menstrual phases backwards to ensure the session date occurs during one of the estimated phases. Contrary, if participants have their expected next menstruation date before the experiment session date, we estimate their menstrual phases forward (11 out 166 subjects needed a forward estimation).

\footnotetext{
${ }^{24}$ We proceed in the second and third steps with the premenstrual and ovulation phases, and not Postmenstrual, since the latter is known to be the phase with the most variation at individual level (Hampson and Young, 2008).
} 


\section{A2. Descriptive statistics}

Table 4.8: Descriptive statistics

\begin{tabular}{|c|c|c|c|c|c|c|}
\hline & \multicolumn{4}{|c|}{ Non-contraceptive takers } & \multicolumn{2}{|c|}{ Contraceptive takers } \\
\hline & $\begin{array}{c}\text { Menstrual } \\
(\mathrm{n}=17)\end{array}$ & $\begin{array}{c}\text { Postmens. } \\
(n=19)\end{array}$ & $\begin{array}{l}\text { Ovulat. } \\
(\mathrm{n}=20)\end{array}$ & $\begin{array}{c}\text { Premens. } \\
(\mathrm{n}=41)\end{array}$ & $\begin{array}{c}\text { Intake } \\
(n=42)\end{array}$ & $\begin{array}{c}\text { Break } \\
(n=27)\end{array}$ \\
\hline Age (mean) & $22.6 \pm 3.2$ & $21.3 \pm 2.1$ & $21.5 \pm 3.2$ & $21.2 \pm 2.6$ & $21.1 \pm 1.9$ & $20.9 \pm 2.4$ \\
\hline Nationality-no. & & & & & & \\
\hline Dutch/Germ/Belg. & $14(82 \%)$ & $14(26 \%)$ & $12(60 \%)$ & $30(73 \%)$ & $38(90 \%)$ & $25(93 \%)$ \\
\hline Other & $3(18 \%)$ & $5(74 \%)$ & $8(40 \%)$ & $11(27 \%)$ & $4(10 \%)$ & $2(7 \%)$ \\
\hline Studies-no. & & & & & & \\
\hline Econ. related & $7(49 \%)$ & $11(58 \%)$ & $16(80 \%)$ & $32(78 \%)$ & $31(74 \%)$ & $20(74 \%)$ \\
\hline Other & $10(41 \%)$ & $8(42 \%)$ & $4(20 \%)$ & $9(22 \%)$ & $11(26 \%)$ & $7(26 \%)$ \\
\hline Barg. Role-no. & & & & & & \\
\hline Informed & $8(47 \%)$ & $11(58 \%)$ & $12(60 \%)$ & $21(51 \%)$ & $23(55 \%)$ & $19(70 \%)$ \\
\hline Uninformed & $9(53 \%)$ & $8(42 \%)$ & $8(40 \%)$ & $20(49 \%)$ & $19(45 \%)$ & $8(30 \%)$ \\
\hline
\end{tabular}

Note: plus-minus values are means \pm SD. Percentages are calculated as the number of subjects in the corresponding category over the total number of subjects in the specific menstrual phase.

Table 4.8 displays the demographics statistics such as age, nationality, education and role during the bargaining game for both non-contraceptive and contraceptive takers across their respective menstrual phases. Overall, we observe that women from different menstrual phases in our sample have similar characteristics, which ensures they do not differ in any other dimension apart from being in a different menstrual phase. Lastly, the last two rows of Table 3 show the distribution of subjects across the two roles assigned during the bargaining task. 


\section{A3. Additional analysis non-pill takers}

\section{Interactions phases and risk preferences}

Table 4.9: Initial offers and mean relative concession rates for informed players.

\begin{tabular}{|c|c|c|c|c|}
\hline & \multicolumn{2}{|c|}{ Initial offer } & \multicolumn{2}{|c|}{ Concession rate } \\
\hline & (1) & (2) & (3) & (4) \\
\hline Menstrual & & $\begin{array}{c}-1.301 \\
(0.848)\end{array}$ & & $\begin{array}{c}-0.00235 \\
(0.0685)\end{array}$ \\
\hline Postmenstrual & & $\begin{array}{c}0.172 \\
(0.790)\end{array}$ & & $\begin{array}{l}-0.0313 \\
(0.142)\end{array}$ \\
\hline Ovulation & $\begin{array}{c}-1.139^{* *} \\
(0.543)\end{array}$ & $\begin{array}{c}-1.321^{* *} \\
(0.571)\end{array}$ & $\begin{array}{c}-0.0272 \\
(0.0615)\end{array}$ & $\begin{array}{l}-0.0409 \\
(0.0510)\end{array}$ \\
\hline Menstrual $\times$ Risk Aversion & & $\begin{array}{l}-0.966 \\
(1.207)\end{array}$ & & $\begin{array}{l}-0.0770 \\
(0.0849)\end{array}$ \\
\hline Postmenstrual $\times$ Risk Aversion & & $\begin{array}{c}0.318 \\
(0.555)\end{array}$ & & $\begin{array}{c}0.0300 \\
(0.0631)\end{array}$ \\
\hline Ovulation $\times$ Risk Aversion & $\begin{array}{l}0.0786 \\
(0.282)\end{array}$ & $\begin{array}{c}0.253 \\
(0.436)\end{array}$ & $\begin{array}{c}-0.0277 \\
(0.0419)\end{array}$ & $\begin{array}{l}-0.00864 \\
(0.0522)\end{array}$ \\
\hline Pie size & $\begin{array}{l}0.205^{* * *} \\
(0.0224)\end{array}$ & $\begin{array}{l}0.204^{* * *} \\
(0.0227)\end{array}$ & $\begin{array}{l}-0.00262 \\
(0.00697)\end{array}$ & $\begin{array}{c}-0.00252 \\
(0.00689)\end{array}$ \\
\hline Bargaining round & $\begin{array}{c}-0.119^{* * *} \\
(0.0276)\end{array}$ & $\begin{array}{c}-0.119^{* * *} \\
(0.0277)\end{array}$ & $\begin{array}{l}0.00983 \\
(0.0134)\end{array}$ & $\begin{array}{l}0.00991 \\
(0.0137)\end{array}$ \\
\hline Risk Aversion & $\begin{array}{l}-0.327^{*} \\
(0.180)\end{array}$ & $\begin{array}{l}-0.521 \\
(0.422)\end{array}$ & $\begin{array}{c}0.0139 \\
(0.0258)\end{array}$ & $\begin{array}{l}-0.00492 \\
(0.0505)\end{array}$ \\
\hline Social Preferences & $\begin{array}{c}0.212 \\
(0.135)\end{array}$ & $\begin{array}{c}0.253 \\
(0.168)\end{array}$ & $\begin{array}{c}-0.0000855 \\
(0.0262)\end{array}$ & $\begin{array}{c}-0.000769 \\
(0.0287)\end{array}$ \\
\hline Constant & $\begin{array}{c}0.588 \\
(2.484)\end{array}$ & $\begin{array}{c}0.770 \\
(3.228)\end{array}$ & $\begin{array}{c}0.400 \\
(0.451)\end{array}$ & $\begin{array}{c}0.514 \\
(0.583)\end{array}$ \\
\hline Demographics & $\checkmark$ & $\checkmark$ & $\checkmark$ & $\checkmark$ \\
\hline Observations & 520 & 520 & 475 & 475 \\
\hline R-squared & 0.348 & 0.370 & 0.0156 & 0.0169 \\
\hline
\end{tabular}

Note: $* p<0.10, * * p<0.05, * * * p<0.01$. Standard errors are clustered at the matching group level (in parentheses). Post-estimation Bonferroni-corrected Wald tests for ovulation versus the menstrual and postmenstrual phases with the effect sizes and the corresponding p-values [in parentheses]. The dependent variable is initial offers in models 1 and 2 and relative concession rates in models 3 and 4 . Controls for demographics (age, nationality, and educational background) are included in all four models. 
Table 4.10: Initial demands and mean relative concession rates for uninformed players.

\begin{tabular}{|c|c|c|c|c|}
\hline & \multicolumn{2}{|c|}{ Initial offer } & \multicolumn{2}{|c|}{ Concession rate } \\
\hline & (1) & (2) & (3) & (4) \\
\hline Menstrual & & $\begin{array}{l}-1.207 \\
(1.461)\end{array}$ & & $\begin{array}{l}-0.0140 \\
(0.145)\end{array}$ \\
\hline Postmenstrual & & $\begin{array}{c}-2.144 \\
(1.255)\end{array}$ & & $\begin{array}{c}0.0540 \\
(0.0599)\end{array}$ \\
\hline Ovulation & $\begin{array}{c}-1.603 \\
(1.156)\end{array}$ & $\begin{array}{c}-1.914 \\
(1.461)\end{array}$ & $\begin{array}{c}0.105 \\
(0.0868)\end{array}$ & $\begin{array}{c}0.112 \\
(0.101)\end{array}$ \\
\hline Menstrual $\times$ Risk Aversion & & $\begin{array}{c}-0.301 \\
(0.796)\end{array}$ & & $\begin{array}{c}0.0281 \\
(0.0455)\end{array}$ \\
\hline Postmenstrual $\times$ Risk Aversion & & $\begin{array}{c}-4.651 \\
(2.970)\end{array}$ & & $\begin{array}{c}-0.0398 \\
(0.0491)\end{array}$ \\
\hline Ovulation $\times$ Risk Aversion & $\begin{array}{c}0.507 \\
(3.636)\end{array}$ & $\begin{array}{c}0.407 \\
(3.586)\end{array}$ & $\begin{array}{l}-0.0763 \\
(0.225)\end{array}$ & $\begin{array}{l}-0.0667 \\
(0.231)\end{array}$ \\
\hline Pie size & $\begin{array}{c}0.0111 \\
(0.0271)\end{array}$ & $\begin{array}{c}0.0134 \\
(0.0261)\end{array}$ & $\begin{array}{l}-0.00437 \\
(0.00436)\end{array}$ & $\begin{array}{c}-0.00384 \\
(0.00445)\end{array}$ \\
\hline Bargaining round & $\begin{array}{l}0.210^{* * *} \\
(0.0600)\end{array}$ & $\begin{array}{l}0.210^{* * *} \\
(0.0605)\end{array}$ & $\begin{array}{l}0.00722 \\
(0.0213)\end{array}$ & $\begin{array}{l}0.00709 \\
(0.0214)\end{array}$ \\
\hline Risk Aversion & $\begin{array}{l}0.758^{*} \\
(0.425)\end{array}$ & $\begin{array}{c}0.828^{*} \\
(0.424)\end{array}$ & $\begin{array}{l}-0.00519 \\
(0.00965)\end{array}$ & $\begin{array}{l}-0.00726 \\
(0.0109)\end{array}$ \\
\hline Social Preferences & $\begin{array}{l}-0.602 \\
(0.568)\end{array}$ & $\begin{array}{l}-0.546 \\
(0.594)\end{array}$ & $\begin{array}{c}0.0304 \\
(0.0331)\end{array}$ & $\begin{array}{c}0.0294 \\
(0.0328)\end{array}$ \\
\hline Constant & $\begin{array}{l}8.766^{*} \\
(4.952)\end{array}$ & $\begin{array}{l}8.879^{*} \\
(4.991)\end{array}$ & $\begin{array}{c}0.764 \\
(0.560)\end{array}$ & $\begin{array}{c}0.718 \\
(0.569)\end{array}$ \\
\hline Demographics & $\checkmark$ & $\checkmark$ & $\checkmark$ & $\checkmark$ \\
\hline Observations & 450 & 450 & 413 & 413 \\
\hline R-squared & 0.150 & 0.181 & 0.0159 & 0.0183 \\
\hline
\end{tabular}

Note: $* p<0.10, * * p<0.05, * * * p<0.01$. Standard errors are clustered at the matching group level (in parentheses). Post-estimation Bonferroni-corrected Wald tests for ovulation versus the menstrual and postmenstrual phases with the effect sizes and the corresponding p-values [in parentheses]. The dependent variable is initial offers in models 1 and 2 and relative concession rates in models 3 and 4. Controls for demographics (age, nationality, and educational background) are included in all four models. 
Table 4.11: Informed player's un/conditional payoffs and deal rates with interactions between menstrual phases and risk preferences.

\begin{tabular}{|c|c|c|c|c|c|c|}
\hline & \multicolumn{2}{|c|}{ Unconditional Payoffs } & \multicolumn{2}{|c|}{ Conditional Payoffs } & \multicolumn{2}{|c|}{ Deals } \\
\hline & (1) & (2) & (3) & (4) & (5) & (6) \\
\hline Menstrual & & $\begin{array}{c}0.439 \\
(0.335)\end{array}$ & & $\begin{array}{c}0.689 \\
(0.588)\end{array}$ & & $\begin{array}{c}-0.074 \\
(0.072)\end{array}$ \\
\hline Post-Menstrual & & $\begin{array}{c}-0.100 \\
(0.468)\end{array}$ & & $\begin{array}{c}-0.379 \\
(0.655)\end{array}$ & & $\begin{array}{c}-0.013 \\
(0.052)\end{array}$ \\
\hline Ovulation & $\begin{array}{c}0.758 \\
(0.574)\end{array}$ & $\begin{array}{c}0.788 \\
(0.599)\end{array}$ & $\begin{array}{l}1.113^{* *} \\
(0.513)\end{array}$ & $\begin{array}{l}1.074^{* *} \\
(0.522)\end{array}$ & $\begin{array}{c}0.004 \\
(0.054)\end{array}$ & $\begin{array}{c}-0.016 \\
(0.064)\end{array}$ \\
\hline Menstrual $\times$ Risk Aversion & & $\begin{array}{c}1.616^{* * *} \\
(0.473)\end{array}$ & & $\begin{array}{c}1.385 \\
(1.060)\end{array}$ & & $\begin{array}{c}0.033 \\
(0.075)\end{array}$ \\
\hline Post-Menstrual $\times$ Risk Aversion & & $\begin{array}{c}-0.183 \\
(0.281)\end{array}$ & & $\begin{array}{l}-0.073 \\
(0.473)\end{array}$ & & $\begin{array}{c}0.025 \\
(0.043)\end{array}$ \\
\hline Ovulation $\times$ Risk Aversion & $\begin{array}{c}0.044 \\
(0.224)\end{array}$ & $\begin{array}{c}-0.038 \\
(0.314)\end{array}$ & $\begin{array}{c}0.191 \\
(0.219)\end{array}$ & $\begin{array}{c}0.185 \\
(0.316)\end{array}$ & $\begin{array}{c}-0.030 \\
(0.021)\end{array}$ & $\begin{array}{l}-0.013 \\
(0.035)\end{array}$ \\
\hline Pie size & $\begin{array}{c}0.618^{* * *} \\
(0.029)\end{array}$ & $\begin{array}{c}0.619^{* * *} \\
(0.029)\end{array}$ & $\begin{array}{c}0.713^{* * *} \\
(0.024)\end{array}$ & $\begin{array}{c}0.713^{* * *} \\
(0.024)\end{array}$ & $\begin{array}{c}0.015^{* * *} \\
(0.003)\end{array}$ & $\begin{array}{c}0.015^{* * *} \\
(0.003)\end{array}$ \\
\hline Bargaining round & $\begin{array}{l}0.171^{* *} \\
(0.075)\end{array}$ & $\begin{array}{l}0.169^{* *} \\
(0.075)\end{array}$ & $\begin{array}{c}0.028 \\
(0.052)\end{array}$ & $\begin{array}{c}0.038 \\
(0.053)\end{array}$ & $\begin{array}{c}0.013^{*} \\
(0.007)\end{array}$ & $\begin{array}{c}0.013^{*} \\
(0.007)\end{array}$ \\
\hline Initial Position Partner & $\begin{array}{c}-0.142^{* *} \\
(0.055)\end{array}$ & $\begin{array}{c}-0.135^{* *} \\
(0.056)\end{array}$ & $\begin{array}{l}-0.023 \\
(0.053)\end{array}$ & $\begin{array}{l}-0.024 \\
(0.055)\end{array}$ & $\begin{array}{c}-0.018^{* * *} \\
(0.005)\end{array}$ & $\begin{array}{c}-0.017^{* * *} \\
(0.005)\end{array}$ \\
\hline Risk Aversion & $\begin{array}{c}0.488^{* * *} \\
(0.117)\end{array}$ & $\begin{array}{l}0.561^{* *} \\
(0.241)\end{array}$ & $\begin{array}{c}0.305^{*} \\
(0.160)\end{array}$ & $\begin{array}{c}0.313 \\
(0.319)\end{array}$ & $\begin{array}{c}0.040^{* * *} \\
(0.013)\end{array}$ & $\begin{array}{c}0.021 \\
(0.034)\end{array}$ \\
\hline Social Preferences & $\begin{array}{l}-0.078 \\
(0.112)\end{array}$ & $\begin{array}{c}-0.120 \\
(0.117)\end{array}$ & $\begin{array}{l}-0.262^{*} \\
(0.134)\end{array}$ & $\begin{array}{l}-0.310^{*} \\
(0.158)\end{array}$ & $\begin{array}{c}-0.002 \\
(0.013)\end{array}$ & $\begin{array}{l}-0.003 \\
(0.012)\end{array}$ \\
\hline Constant & $\begin{array}{l}-0.565 \\
(1.224)\end{array}$ & $\begin{array}{c}-1.202 \\
(1.399)\end{array}$ & $\begin{array}{l}-0.355 \\
(1.888)\end{array}$ & $\begin{array}{l}-0.393 \\
(2.398)\end{array}$ & $\begin{array}{l}0.530^{* *} \\
(0.201)\end{array}$ & $\begin{array}{l}0.541^{* *} \\
(0.246)\end{array}$ \\
\hline Observations & 520.000 & 520.000 & 364.000 & 364.000 & 520.000 & 520.000 \\
\hline R-squared & 0.518 & 0.520 & 0.822 & 0.825 & 0.101 & 0.104 \\
\hline
\end{tabular}

Note: $* p<0.10,{ }^{* *} p<0.05, * * * p<0.01$. Standard errors are clustered at the matching group level (in parentheses). Post-estimation Bonferroni-corrected Wald tests for ovulation versus the menstrual and postmenstrual phases with the effect size and the corresponding p-values [in parentheses]. The dependent variable is unconditional payoffs in models 1 and 2, payoffs conditional on a deal in models 3 and 4, and deal rates in models 5 and 6 . Controls for demographics (age, nationality, and educational background) are included in all six models. 
Table 4.12: Uninformed player's un/conditional payoffs and deal rates with interactions between menstrual phases and risk preferences.

\begin{tabular}{|c|c|c|c|c|c|c|}
\hline & \multicolumn{2}{|c|}{ Unconditional Payoffs } & \multicolumn{2}{|c|}{ Conditional Payoffs } & \multicolumn{2}{|c|}{ Deals } \\
\hline & (1) & (2) & (3) & (4) & (5) & (6) \\
\hline Menstrual & & $\begin{array}{c}-0.982^{* *} \\
(0.394)\end{array}$ & & $\begin{array}{l}-0.565 \\
(0.536)\end{array}$ & & $\begin{array}{c}-0.086 \\
(0.090)\end{array}$ \\
\hline Post-Menstrual & & $\begin{array}{c}-0.050 \\
(0.378)\end{array}$ & & $\begin{array}{l}-0.342 \\
(0.310)\end{array}$ & & $\begin{array}{c}-0.010 \\
(0.099)\end{array}$ \\
\hline Ovulation & $\begin{array}{l}1.220^{* *} \\
(0.473)\end{array}$ & $\begin{array}{l}1.105^{* *} \\
(0.469)\end{array}$ & $\begin{array}{c}0.073 \\
(0.327)\end{array}$ & $\begin{array}{c}0.065 \\
(0.367)\end{array}$ & $\begin{array}{l}0.170^{* *} \\
(0.072)\end{array}$ & $\begin{array}{l}0.154^{* *} \\
(0.071)\end{array}$ \\
\hline Menstrual $\times$ Risk Aversion & & $\begin{array}{c}-0.384^{* *} \\
(0.146)\end{array}$ & & $\begin{array}{c}-0.633^{* * *} \\
(0.215)\end{array}$ & & $\begin{array}{c}-0.022 \\
(0.033)\end{array}$ \\
\hline Post-Menstrual $\times$ Risk Aversion & & $\begin{array}{c}-0.784 \\
(0.502)\end{array}$ & & $\begin{array}{l}-1.335 \\
(0.921)\end{array}$ & & $\begin{array}{c}-0.028 \\
(0.157)\end{array}$ \\
\hline Ovulation $\times$ Risk Aversion & $\begin{array}{c}-1.389 \\
(1.133)\end{array}$ & $\begin{array}{c}-1.353 \\
(1.164)\end{array}$ & $\begin{array}{c}-0.297 \\
(0.743)\end{array}$ & $\begin{array}{c}-0.388 \\
(0.700)\end{array}$ & $\begin{array}{c}-0.163 \\
(0.158)\end{array}$ & $\begin{array}{c}-0.157 \\
(0.163)\end{array}$ \\
\hline Pie size & $\begin{array}{c}0.119^{* * *} \\
(0.024)\end{array}$ & $\begin{array}{c}0.119^{* * *} \\
(0.025)\end{array}$ & $\begin{array}{c}0.132^{* * *} \\
(0.021)\end{array}$ & $\begin{array}{c}0.131^{* * *} \\
(0.021)\end{array}$ & $\begin{array}{c}0.007^{*} \\
(0.004)\end{array}$ & $\begin{array}{c}0.007^{*} \\
(0.004)\end{array}$ \\
\hline Bargaining round & $\begin{array}{l}0.112^{* *} \\
(0.049)\end{array}$ & $\begin{array}{c}0.113^{* *} \\
(0.050)\end{array}$ & $\begin{array}{l}0.070^{* *} \\
(0.028)\end{array}$ & $\begin{array}{l}0.071^{* *} \\
(0.027)\end{array}$ & $\begin{array}{c}0.013 \\
(0.008)\end{array}$ & $\begin{array}{c}0.013 \\
(0.008)\end{array}$ \\
\hline Initial Position Partner & $\begin{array}{c}0.708^{* * *} \\
(0.078)\end{array}$ & $\begin{array}{c}0.712^{* * *} \\
(0.079)\end{array}$ & $\begin{array}{c}0.733^{* * *} \\
(0.056)\end{array}$ & $\begin{array}{c}0.741^{* * *} \\
(0.059)\end{array}$ & $\begin{array}{l}0.019^{* *} \\
(0.008)\end{array}$ & $\begin{array}{l}0.019^{* *} \\
(0.008)\end{array}$ \\
\hline Risk Aversion & $\begin{array}{c}-0.000 \\
(0.061)\end{array}$ & $\begin{array}{c}0.034 \\
(0.062)\end{array}$ & $\begin{array}{c}-0.079 \\
(0.115)\end{array}$ & $\begin{array}{c}0.004 \\
(0.095)\end{array}$ & $\begin{array}{c}0.008 \\
(0.008)\end{array}$ & $\begin{array}{c}0.008 \\
(0.008)\end{array}$ \\
\hline Social Preferences & $\begin{array}{c}-0.214 \\
(0.133)\end{array}$ & $\begin{array}{c}-0.118 \\
(0.086)\end{array}$ & $\begin{array}{c}-0.263 \\
(0.171)\end{array}$ & $\begin{array}{c}-0.184 \\
(0.170)\end{array}$ & $\begin{array}{c}0.000 \\
(0.022)\end{array}$ & $\begin{array}{c}0.007 \\
(0.021)\end{array}$ \\
\hline Constant & $\begin{array}{c}0.989 \\
(1.379)\end{array}$ & $\begin{array}{c}0.630 \\
(1.326)\end{array}$ & $\begin{array}{c}0.747 \\
(1.311)\end{array}$ & $\begin{array}{c}0.561 \\
(1.310)\end{array}$ & $\begin{array}{c}0.825^{* * *} \\
(0.180)\end{array}$ & $\begin{array}{c}0.804^{* * *} \\
(0.192)\end{array}$ \\
\hline Observations & 450.000 & 450.000 & 319.000 & 319.000 & 450.000 & 450.000 \\
\hline R-squared & 0.487 & 0.496 & 0.738 & 0.747 & 0.079 & 0.083 \\
\hline
\end{tabular}

Note: $* p<0.10, * * p<0.05, * * * p<0.01$. Standard errors are clustered at the matching group level (in parentheses). Post-estimation Bonferroni-corrected Wald tests for ovulation versus the menstrual and postmenstrual phases with the effect size and the corresponding p-values [in parentheses]. The dependent variable is unconditional payoffs in models 1 and 2, payoffs conditional on a deal in models 3 and 4 , and deal rates in models 5 and 6. Controls for demographics (age, nationality, and educational background) are included in all six models. 


\section{Mean relative concession rates}

Table 4.13: Mean relative concession rates for both players (Excluding initial offer/demand).

\begin{tabular}{|c|c|c|c|c|}
\hline & \multicolumn{2}{|c|}{ Informed player } & \multicolumn{2}{|c|}{ Uninformed player } \\
\hline & (1) & (2) & (3) & (4) \\
\hline Menstrual & & $\begin{array}{c}0.0515 \\
(0.0652)\end{array}$ & & $\begin{array}{c}0.198 \\
(0.156)\end{array}$ \\
\hline Postmenstrual & & $\begin{array}{l}-0.221 \\
(0.256)\end{array}$ & & $\begin{array}{c}0.141 \\
(0.108)\end{array}$ \\
\hline Ovulation & $\begin{array}{l}0.00543 \\
(0.0971)\end{array}$ & $\begin{array}{l}-0.0503 \\
(0.0641)\end{array}$ & $\begin{array}{c}0.127 \\
(0.165)\end{array}$ & $\begin{array}{c}0.198 \\
(0.193)\end{array}$ \\
\hline Pie size & $\begin{array}{l}-0.00267 \\
(0.00955)\end{array}$ & $\begin{array}{c}-0.00188 \\
(0.00881)\end{array}$ & $\begin{array}{c}-0.0126^{*} \\
(0.00707)\end{array}$ & $\begin{array}{c}-0.0131^{*} \\
(0.00723)\end{array}$ \\
\hline Bargaining round & $\begin{array}{l}0.00933 \\
(0.0189)\end{array}$ & $\begin{array}{l}0.00978 \\
(0.0190)\end{array}$ & $\begin{array}{l}0.00711 \\
(0.0270)\end{array}$ & $\begin{array}{l}0.00772 \\
(0.0273)\end{array}$ \\
\hline Risk Aversion & $\begin{array}{c}0.0149 \\
(0.0249)\end{array}$ & $\begin{array}{l}0.00473 \\
(0.0311)\end{array}$ & $\begin{array}{l}-0.0137 \\
(0.0178)\end{array}$ & $\begin{array}{l}-0.00382 \\
(0.0190)\end{array}$ \\
\hline Social Preferences & $\begin{array}{c}-0.0000394 \\
(0.0372)\end{array}$ & $\begin{array}{l}-0.00597 \\
(0.0425)\end{array}$ & $\begin{array}{l}0.00808 \\
(0.0370)\end{array}$ & $\begin{array}{l}-0.00387 \\
(0.0394)\end{array}$ \\
\hline Constant & $\begin{array}{c}0.192 \\
(0.652)\end{array}$ & $\begin{array}{c}0.459 \\
(0.905)\end{array}$ & $\begin{array}{c}1.612 \\
(1.039)\end{array}$ & $\begin{array}{c}1.595 \\
(1.012)\end{array}$ \\
\hline Demographics & $\checkmark$ & $\checkmark$ & $\checkmark$ & $\checkmark$ \\
\hline Observations & 425 & 425 & 353 & 353 \\
\hline R-squared & 0.0110 & 0.0195 & 0.0461 & 0.0521 \\
\hline
\end{tabular}

Note: $* p<0.10, * * p<0.05, * * * p<0.01$. Standard errors are clustered at the matching group level (in parentheses). The dependent variable is relative concession rates for informed players in models 1 and 2 and for uninformed players in models 3 and 4. Controls for demographics (age, nationality, and educational background) are included in all four models.

Table 4.14: Spearman rank-order correlations of Mean relative concession rates for between both players (excluding initial offer/demand)

\begin{tabular}{ccccc}
\hline & Menstrual & Postmenstrual & Ovulation & Premenstrual \\
\hline \multirow{3}{*}{ Informed player } & -0.47 & -0.706 & -0.49 & -0.49 \\
& $(\mathrm{p}=0.232)$ & $(\mathrm{p}=0.015)$ & $(\mathrm{p}=0.100)$ & $(\mathrm{p}=0.023)$ \\
& $\mathrm{n}=8$ & $\mathrm{n}=11$ & $\mathrm{n}=12$ & $\mathrm{n}=21$ \\
\hline \multirow{3}{*}{ Uninformed player } & -0.80 & -0.57 & -0.64 & -0.34 \\
& $(\mathrm{p}=0.009)$ & $(\mathrm{p}=0.139)$ & $(\mathrm{p}=0.085)$ & $(\mathrm{p}=0.140)$ \\
& $\mathrm{n}=9$ & $\mathrm{n}=8$ & $\mathrm{n}=8$ & $\mathrm{n}=20$ \\
\hline
\end{tabular}




\section{Risk and Social Preferences for Non-pill takers}

Table 4.15: Risk aversion for both type of players

\begin{tabular}{lccccc}
\hline & \multicolumn{2}{c}{ Informed player } & & \multicolumn{2}{c}{ Uninformed player } \\
\cline { 2 - 3 } \cline { 6 - 6 } & $(1)$ & $(2)$ & & $(3)$ & $(4)$ \\
\hline Menstrual & & $-0.746^{* *}$ & & -0.598 \\
& & $(0.341)$ & & $(0.530)$ \\
Postmenstrual & & $-0.944^{*}$ & & -0.503 \\
& & $(0.546)$ & & $(0.446)$ \\
Ovulation & 0.169 & -0.225 & & 0.178 & -0.0479 \\
& $(0.581)$ & $(0.568)$ & & $(0.293)$ & $(0.407)$ \\
Constant & 0.460 & 1.131 & & 1.640 & 1.820 \\
& $(1.932)$ & $(1.549)$ & & $(1.401)$ & $(1.413)$ \\
Demographics & $\checkmark$ & $\checkmark$ & & $\checkmark$ \\
\hline Observations & 520 & 520 & 450 & 450 \\
R-squared & 0.0383 & 0.111 & 0.107 & 0.134 \\
\hline
\end{tabular}

Note: $* p<0.10, * * p<0.05, * * * p<0.01$. Standard errors (in parentheses). The dependent variable for all four models is the risk aversion coefficient. The explanatory variables are pie size, bargaining round, risk aversion and social preferences. Demographics (age, nationality, and educational background) are included in all four models. Standard errors are clustered at matching group level.

Table 4.15 shows the regression analysis for risk aversion levels for informed and uninformed players. The results provided in Table 4.15 suggest that informed players are less averse to the risk during the menstrual and Postmenstrual phases when compared to the premenstrual phase. For uninformed players, we do not observe significant changes in the risk aversion levels across the menstrual cycle.

Result: Informed players are less risk averse when they are in the menstrual and Postmenstrual phase versus the premenstrual. Uninformed players levels of risk aversion do not change across the menstrual cycle.

For social preferences, we do not observe any significant variation in the level on benevolence between the menstrual phases for both types of players.

Result: Social preferences do not vary across the menstrual cycle in their . 
Table 4.16: Benevolence levels for both type of players

\begin{tabular}{lccccc}
\hline & \multicolumn{2}{c}{ Informed player } & \multicolumn{2}{c}{ Uninformed player } \\
\cline { 2 - 3 } \cline { 6 - 6 } & $(1)$ & $(2)$ & & $(3)$ & $(4)$ \\
\hline Menstrual & & 0.363 & & 0.545 \\
& & $(0.659)$ & & $(0.724)$ \\
Postmenstrual & & -0.187 & & 0.0603 \\
& & $(0.511)$ & & $(0.393)$ \\
Ovulation & 0.0395 & 0.0425 & & -0.0992 & 0.0256 \\
& $(0.357)$ & $(0.415)$ & & $(0.424)$ & $(0.391)$ \\
Constant & $5.776^{* *}$ & $6.039^{* *}$ & & $3.035^{* *}$ & $3.039^{* *}$ \\
& $(2.616)$ & $(2.525)$ & & $(1.279)$ & $(1.244)$ \\
Demographics & $\checkmark$ & $\checkmark$ & & $\checkmark$ \\
\hline Observations & 520 & 520 & & 450 & 450 \\
R-squared & 0.0130 & 0.0277 & & 0.274 & 0.295 \\
\hline
\end{tabular}

Note: $* p<0.10, * * p<0.05, * * * p<0.01$. Standard errors (in parentheses). The dependent variable in the four models is the level of benevolence of the participant. The explanatory variables are pie size, bargaining round, risk aversion and social preferences. Demographics (age, nationality, and educational background) are included in all four models. Standard errors are clustered at matching group level. 


\section{A4. Different length of phases - Non-contraceptive takers}

In this subsection we run the whole analysis for a different length of the menstrual phase. Specifically, the ovulation phase is considered to last 3 days (instead of 5 days).

Table 4.17: Initial offers and mean relative concession rates for informed players.

\begin{tabular}{|c|c|c|c|c|}
\hline & \multicolumn{2}{|c|}{ Initial offer } & \multicolumn{2}{|c|}{ Concession rate } \\
\hline & (1) & (2) & (3) & (4) \\
\hline Menstrual & & $\begin{array}{c}-0.575 \\
(0.746)\end{array}$ & & $\begin{array}{c}0.0391 \\
(0.0477)\end{array}$ \\
\hline Postmenstrual & & $\begin{array}{c}0.456 \\
(0.733)\end{array}$ & & $\begin{array}{l}-0.0170 \\
(0.120)\end{array}$ \\
\hline Ovulation & $\begin{array}{c}-0.685 \\
(0.676)\end{array}$ & $\begin{array}{l}-0.590 \\
(0.675)\end{array}$ & $\begin{array}{c}-0.0626 \\
(0.0758)\end{array}$ & $\begin{array}{c}-0.0650 \\
(0.0660)\end{array}$ \\
\hline Pie size & $\begin{array}{l}0.203^{* * *} \\
(0.0223)\end{array}$ & $\begin{array}{l}0.202^{* * *} \\
(0.0225)\end{array}$ & $\begin{array}{c}-0.00297 \\
(0.00693)\end{array}$ & $\begin{array}{c}-0.00286 \\
(0.00687)\end{array}$ \\
\hline Bargaining round & $\begin{array}{c}-0.119^{* * *} \\
(0.0275)\end{array}$ & $\begin{array}{c}-0.119^{* * *} \\
(0.0275)\end{array}$ & $\begin{array}{l}0.00992 \\
(0.0134)\end{array}$ & $\begin{array}{c}0.0101 \\
(0.0136)\end{array}$ \\
\hline Risk Aversion & $\begin{array}{c}-0.305^{* *} \\
(0.126)\end{array}$ & $\begin{array}{c}-0.297^{* *} \\
(0.136)\end{array}$ & $\begin{array}{l}0.00223 \\
(0.0201)\end{array}$ & $\begin{array}{l}0.00256 \\
(0.0197)\end{array}$ \\
\hline Social Preferences & $\begin{array}{c}0.188 \\
(0.143)\end{array}$ & $\begin{array}{c}0.217 \\
(0.156)\end{array}$ & $\begin{array}{l}0.00309 \\
(0.0232)\end{array}$ & $\begin{array}{l}0.00169 \\
(0.0261)\end{array}$ \\
\hline Constant & $\begin{array}{c}0.182 \\
(2.586)\end{array}$ & $\begin{array}{c}-0.590 \\
(2.894)\end{array}$ & $\begin{array}{c}0.418 \\
(0.464)\end{array}$ & $\begin{array}{c}0.454 \\
(0.507)\end{array}$ \\
\hline Demographics & $\checkmark$ & $\checkmark$ & $\checkmark$ & $\checkmark$ \\
\hline Observations & 520 & 520 & 475 & 475 \\
\hline R-squared & 0.328 & 0.339 & 0.0153 & 0.0158 \\
\hline
\end{tabular}

Note: $* p<0.10, * * p<0.05$, *** $p<0.01$. Standard errors (in parentheses). The dependent variable for Models 1 and 2 is initial offer and relative concession rates for Models 3 and 4 . The explanatory variables are pie size, bargaining round, risk aversion and social preferences. Demographics (age, nationality, and educational background) are included in all four models. Standard errors are clustered at matching group level. 
Table 4.18: Initial demands and mean relative concession rates for uninformed players.

\begin{tabular}{|c|c|c|c|c|}
\hline & \multicolumn{2}{|c|}{ Initial demand } & \multicolumn{2}{|c|}{ Concession rate } \\
\hline & (1) & (2) & (3) & (4) \\
\hline Menstrual & & $\begin{array}{c}-1.061 \\
(1.368)\end{array}$ & & $\begin{array}{l}-0.0315 \\
(0.125)\end{array}$ \\
\hline Postmenstrual & & $\begin{array}{c}-0.859 \\
(1.564)\end{array}$ & & $\begin{array}{c}0.0748 \\
(0.0498)\end{array}$ \\
\hline Ovulation & $\begin{array}{c}-1.040 \\
(1.445)\end{array}$ & $\begin{array}{c}-1.410 \\
(1.733)\end{array}$ & $\begin{array}{c}0.128 \\
(0.101)\end{array}$ & $\begin{array}{c}0.141 \\
(0.112)\end{array}$ \\
\hline Pie size & $\begin{array}{c}0.0104 \\
(0.0255)\end{array}$ & $\begin{array}{c}0.0101 \\
(0.0242)\end{array}$ & $\begin{array}{c}-0.00442 \\
(0.00444)\end{array}$ & $\begin{array}{c}-0.00396 \\
(0.00447)\end{array}$ \\
\hline Bargaining round & $\begin{array}{l}0.210^{* * *} \\
(0.0599)\end{array}$ & $\begin{array}{l}0.210^{* * *} \\
(0.0601)\end{array}$ & $\begin{array}{l}0.00735 \\
(0.0211)\end{array}$ & $\begin{array}{c}0.00703 \\
(0.0212)\end{array}$ \\
\hline Risk Aversion & $\begin{array}{l}0.758^{*} \\
(0.431)\end{array}$ & $\begin{array}{c}0.701 \\
(0.426)\end{array}$ & $\begin{array}{c}-0.00674 \\
(0.00960)\end{array}$ & $\begin{array}{c}-0.00616 \\
(0.0107)\end{array}$ \\
\hline Social Preferences & $\begin{array}{l}-0.629 \\
(0.579)\end{array}$ & $\begin{array}{c}-0.581 \\
(0.558)\end{array}$ & $\begin{array}{c}0.0350 \\
(0.0322)\end{array}$ & $\begin{array}{c}0.0386 \\
(0.0292)\end{array}$ \\
\hline Constant & $\begin{array}{l}9.368^{*} \\
(4.943)\end{array}$ & $\begin{array}{l}9.680^{*} \\
(4.965)\end{array}$ & $\begin{array}{c}0.699 \\
(0.526)\end{array}$ & $\begin{array}{c}0.646 \\
(0.531)\end{array}$ \\
\hline Demographics & $\checkmark$ & $\checkmark$ & $\checkmark$ & $\checkmark$ \\
\hline Observations & 450 & 450 & 413 & 413 \\
\hline R-squared & 0.140 & 0.148 & 0.0166 & 0.0190 \\
\hline
\end{tabular}

Note: $* p<0.10, * * p<0.05$, *** $p<0.01$. Standard errors (in parentheses). The dependent variable for Models 1 and 2 is initial offer and relative concession rates for Models 3 and 4. The explanatory variables are pie size, bargaining round, risk aversion and social preferences. Demographics (age, nationality, and educational background) are included in all four models. Standard errors are clustered at matching group level. 
Table 4.19: Informed players' un/conditinal payoffs and dela rates

\begin{tabular}{|c|c|c|c|c|c|c|}
\hline & \multicolumn{2}{|c|}{ Unconditional Payoffs } & \multicolumn{2}{|c|}{ Conditional Payoffs } & \multicolumn{2}{|c|}{ Deal rates } \\
\hline & (1) & (2) & (3) & (4) & (5) & (6) \\
\hline Menstruation & & $\begin{array}{c}-0.217 \\
(0.441)\end{array}$ & & $\begin{array}{c}0.086 \\
(0.554)\end{array}$ & & $\begin{array}{c}-0.076 \\
(0.047)\end{array}$ \\
\hline Postmenstruation & & $\begin{array}{c}-0.272 \\
(0.529)\end{array}$ & & $\begin{array}{c}-0.463 \\
(0.611)\end{array}$ & & $\begin{array}{c}-0.030 \\
(0.050)\end{array}$ \\
\hline Ovulation & $\begin{array}{c}1.023 \\
(0.651)\end{array}$ & $\begin{array}{c}0.927 \\
(0.679)\end{array}$ & $\begin{array}{l}1.489^{* *} \\
(0.615)\end{array}$ & $\begin{array}{l}1.360^{* *} \\
(0.621)\end{array}$ & $\begin{array}{c}0.011 \\
(0.062)\end{array}$ & $\begin{array}{c}-0.003 \\
(0.062)\end{array}$ \\
\hline Pie size & $\begin{array}{c}0.622^{* * *} \\
(0.030)\end{array}$ & $\begin{array}{c}0.622^{* * *} \\
(0.030)\end{array}$ & $\begin{array}{c}0.720^{* * *} \\
(0.024)\end{array}$ & $\begin{array}{c}0.721^{* * *} \\
(0.024)\end{array}$ & $\begin{array}{c}0.015^{* * *} \\
(0.003)\end{array}$ & $\begin{array}{c}0.015^{* * *} \\
(0.003)\end{array}$ \\
\hline Bargaining round & $\begin{array}{c}0.169^{* *} \\
(0.075)\end{array}$ & $\begin{array}{l}0.169^{* *} \\
(0.075)\end{array}$ & $\begin{array}{c}0.023 \\
(0.053)\end{array}$ & $\begin{array}{c}0.022 \\
(0.052)\end{array}$ & $\begin{array}{l}0.013^{*} \\
(0.007)\end{array}$ & $\begin{array}{l}0.013^{*} \\
(0.007)\end{array}$ \\
\hline Initial Position Partner & $\begin{array}{c}-0.136^{* *} \\
(0.054)\end{array}$ & $\begin{array}{c}-0.134^{* *} \\
(0.056)\end{array}$ & $\begin{array}{l}-0.007 \\
(0.052)\end{array}$ & $\begin{array}{l}-0.009 \\
(0.055)\end{array}$ & $\begin{array}{c}-0.018^{* * *} \\
(0.005)\end{array}$ & $\begin{array}{c}-0.017^{* * *} \\
(0.005)\end{array}$ \\
\hline Risk Aversion & $\begin{array}{c}0.508^{* * *} \\
(0.130)\end{array}$ & $\begin{array}{c}0.485^{* * *} \\
(0.135)\end{array}$ & $\begin{array}{l}0.387^{* *} \\
(0.140)\end{array}$ & $\begin{array}{l}0.367^{* *} \\
(0.140)\end{array}$ & $\begin{array}{c}0.028^{* * *} \\
(0.010)\end{array}$ & $\begin{array}{l}0.024^{* *} \\
(0.011)\end{array}$ \\
\hline Social Preferences & $\begin{array}{c}-0.067 \\
(0.117)\end{array}$ & $\begin{array}{c}-0.067 \\
(0.118)\end{array}$ & $\begin{array}{l}-0.264^{*} \\
(0.145)\end{array}$ & $\begin{array}{l}-0.279^{*} \\
(0.148)\end{array}$ & $\begin{array}{c}0.002 \\
(0.012)\end{array}$ & $\begin{array}{c}0.004 \\
(0.012)\end{array}$ \\
\hline Constant & $\begin{array}{c}-0.714 \\
(1.255)\end{array}$ & $\begin{array}{c}-0.542 \\
(1.429)\end{array}$ & $\begin{array}{c}-0.587 \\
(2.022)\end{array}$ & $\begin{array}{c}-0.141 \\
(2.133)\end{array}$ & $\begin{array}{l}0.522^{* *} \\
(0.204)\end{array}$ & $\begin{array}{l}0.512^{* *} \\
(0.208)\end{array}$ \\
\hline Observations & 520 & 520 & 364 & 364 & 520 & 520 \\
\hline R-squared & 0.518 & 0.518 & 0.820 & 0.821 & 0.100 & 0.103 \\
\hline
\end{tabular}

Note: $* p<0.10, * * p<0.05, * * *<<0.01$. Standard errors are clustered at the matching group level (in parentheses). Post-estimation Bonferroni-corrected Wald tests for ovulation versus the menstrual and postmenstrual phases with the effect size and the corresponding p-values [in parentheses]. The dependent variable is unconditional payoffs in models 1 and 2, payoffs conditional on a deal in models 3 and 4, and deal rates in models 5 and 6 . Controls for demographics (age, nationality, and educational background) are included in all six models. 
Table 4.20: Uninformed players' un/conditional payoffs and deal rates

\begin{tabular}{|c|c|c|c|c|c|c|}
\hline & \multicolumn{2}{|c|}{ Unconditional Payoffs } & \multicolumn{2}{|c|}{ Conditional Payoffs } & \multicolumn{2}{|c|}{ Deals } \\
\hline & (1) & (2) & (3) & (4) & (5) & (6) \\
\hline Menstruation & & $\begin{array}{c}-0.834^{* *} \\
(0.363)\end{array}$ & & $\begin{array}{c}-0.211 \\
(0.542)\end{array}$ & & $\begin{array}{c}-0.085 \\
(0.082)\end{array}$ \\
\hline Postmenstruation & & $\begin{array}{c}0.315 \\
(0.317)\end{array}$ & & $\begin{array}{c}0.157 \\
(0.368)\end{array}$ & & $\begin{array}{c}0.003 \\
(0.067)\end{array}$ \\
\hline Ovulation & $\begin{array}{c}0.949 \\
(0.569)\end{array}$ & $\begin{array}{c}0.884 \\
(0.562)\end{array}$ & $\begin{array}{c}0.089 \\
(0.353)\end{array}$ & $\begin{array}{c}0.102 \\
(0.378)\end{array}$ & $\begin{array}{c}0.147^{*} \\
(0.084)\end{array}$ & $\begin{array}{c}0.133 \\
(0.084)\end{array}$ \\
\hline Pie size & $\begin{array}{c}0.118^{* * *} \\
(0.024)\end{array}$ & $\begin{array}{c}0.123^{* * *} \\
(0.025)\end{array}$ & $\begin{array}{c}0.133^{* * *} \\
(0.021)\end{array}$ & $\begin{array}{c}0.134^{* * *} \\
(0.022)\end{array}$ & $\begin{array}{l}0.007^{*} \\
(0.004)\end{array}$ & $\begin{array}{c}0.007^{*} \\
(0.004)\end{array}$ \\
\hline Bargaining round & $\begin{array}{l}0.113^{* *} \\
(0.049)\end{array}$ & $\begin{array}{l}0.111^{* *} \\
(0.049)\end{array}$ & $\begin{array}{l}0.070^{* *} \\
(0.028)\end{array}$ & $\begin{array}{l}0.070^{* *} \\
(0.027)\end{array}$ & $\begin{array}{c}0.013 \\
(0.008)\end{array}$ & $\begin{array}{c}0.013 \\
(0.008)\end{array}$ \\
\hline Initial Position Partner & $\begin{array}{c}0.711^{* * *} \\
(0.080)\end{array}$ & $\begin{array}{c}0.703^{* * *} \\
(0.081)\end{array}$ & $\begin{array}{c}0.731^{* * *} \\
(0.056)\end{array}$ & $\begin{array}{c}0.725^{* * *} \\
(0.059)\end{array}$ & $\begin{array}{l}0.019^{* *} \\
(0.008)\end{array}$ & $\begin{array}{c}0.019^{* *} \\
(0.008)\end{array}$ \\
\hline Risk Aversion & $\begin{array}{c}-0.013 \\
(0.064)\end{array}$ & $\begin{array}{c}-0.036 \\
(0.076)\end{array}$ & $\begin{array}{c}-0.083 \\
(0.115)\end{array}$ & $\begin{array}{c}-0.087 \\
(0.116)\end{array}$ & $\begin{array}{c}0.006 \\
(0.009)\end{array}$ & $\begin{array}{c}0.003 \\
(0.010)\end{array}$ \\
\hline Social Preferences & $\begin{array}{c}-0.178 \\
(0.142)\end{array}$ & $\begin{array}{c}-0.128 \\
(0.103)\end{array}$ & $\begin{array}{c}-0.257 \\
(0.178)\end{array}$ & $\begin{array}{c}-0.245 \\
(0.183)\end{array}$ & $\begin{array}{c}0.005 \\
(0.023)\end{array}$ & $\begin{array}{c}0.010 \\
(0.021)\end{array}$ \\
\hline Constant & $\begin{array}{c}0.173 \\
(1.368)\end{array}$ & $\begin{array}{c}-0.099 \\
(1.263)\end{array}$ & $\begin{array}{c}0.613 \\
(1.258)\end{array}$ & $\begin{array}{c}0.497 \\
(1.231)\end{array}$ & $\begin{array}{c}0.719^{* * *} \\
(0.202)\end{array}$ & $\begin{array}{c}0.706^{* * *} \\
(0.204)\end{array}$ \\
\hline Observations & 450.000 & 450.000 & 319.000 & 319.000 & 450.000 & 450.000 \\
\hline R-squared & 0.481 & 0.490 & 0.738 & 0.739 & 0.073 & 0.077 \\
\hline
\end{tabular}

Note: $* p<0.10, * * p<0.05, * * * p<0.01$. Standard errors are clustered at the matching group level (in parentheses). Post-estimation Bonferroni-corrected Wald tests for ovulation versus the menstrual and postmenstrual phases with the effect size and the corresponding p-values [in parentheses]. The dependent variable is unconditional payoffs in models 1 and 2, payoffs conditional on a deal in models 3 and 4, and deal rates in models 5 and 6 . Controls for demographics (age, nationality, and educational background) are included in all six models. 


\section{A5. Contraceptive takers}

Table 4.21: Initial offer/demand and concession rates for informed and uninformed players.

\begin{tabular}{|c|c|c|c|c|}
\hline & \multicolumn{2}{|c|}{ Informed player } & \multicolumn{2}{|c|}{ Uninformed player } \\
\hline & $\begin{array}{c}\text { (1) } \\
\text { Initial offer }\end{array}$ & $\begin{array}{c}(2) \\
\text { Concess rate }\end{array}$ & $\begin{array}{c}\text { (3) } \\
\text { Initial demand }\end{array}$ & $\begin{array}{c}\text { (4) } \\
\text { Concess rate }\end{array}$ \\
\hline Intake & $\begin{array}{l}-0.176 \\
(0.582)\end{array}$ & $\begin{array}{l}-0.0884 \\
(0.0648)\end{array}$ & $\begin{array}{l}-0.364 \\
(0.838)\end{array}$ & $\begin{array}{c}0.0361 \\
(0.0500)\end{array}$ \\
\hline Pie size & $\begin{array}{l}0.271^{* * *} \\
(0.0286)\end{array}$ & $\begin{array}{l}0.0124^{* * *} \\
(0.00236)\end{array}$ & $\begin{array}{c}-0.0443^{* *} \\
(0.0212)\end{array}$ & $\begin{array}{c}-0.00494 \\
(0.00413)\end{array}$ \\
\hline Bargaining round & $\begin{array}{l}-0.215^{* * *} \\
(0.0424)\end{array}$ & $\begin{array}{c}0.00235 \\
(0.00384)\end{array}$ & $\begin{array}{c}0.110^{*} \\
(0.0631)\end{array}$ & $\begin{array}{c}0.00323 \\
(0.00724)\end{array}$ \\
\hline Risk Aversion & $\begin{array}{l}0.196^{*} \\
(0.112)\end{array}$ & $\begin{array}{c}-0.00299 \\
(0.00861)\end{array}$ & $\begin{array}{c}0.128 \\
(0.130)\end{array}$ & $\begin{array}{c}0.0150 \\
(0.0137)\end{array}$ \\
\hline Social Preferences & $\begin{array}{c}-0.276 \\
(0.278)\end{array}$ & $\begin{array}{l}-0.0351 \\
(0.0234)\end{array}$ & $\begin{array}{c}0.229 \\
(0.213)\end{array}$ & $\begin{array}{c}0.0325 \\
(0.0239)\end{array}$ \\
\hline Constant & $\begin{array}{l}7.778^{*} \\
(4.314)\end{array}$ & $\begin{array}{l}0.390^{*} \\
(0.220)\end{array}$ & $\begin{array}{l}-0.169 \\
(6.256)\end{array}$ & $\begin{array}{c}0.322 \\
(0.228)\end{array}$ \\
\hline Demographics & $\checkmark$ & $\checkmark$ & $\checkmark$ & $\checkmark$ \\
\hline $\begin{array}{l}\text { Observations } \\
\text { R-squared }\end{array}$ & $\begin{array}{c}310 \\
0.492\end{array}$ & $\begin{array}{c}278 \\
0.160\end{array}$ & $\begin{array}{c}379 \\
0.150\end{array}$ & $\begin{array}{c}339 \\
0.0549\end{array}$ \\
\hline
\end{tabular}

Note: $* p<0.10, * * p<0.05$, *** $p<0.01$. Standard errors (in parentheses). The dependent variable for Models 1 and 3 is initial offer/demand and relative concession rates for Models 2 and 4. The explanatory variables are the dummy variable Intake which takes the value of one if the participant is in the intake phase and zero otherwise, pie size, bargaining round, risk aversion and social preferences. Demographics (age, nationality, and educational background) are included in all four models. Standard errors are clustered at matching group level. 
Table 4.22: Informed players' un/conditional payoffs and deal rates

\begin{tabular}{|c|c|c|c|}
\hline & Unconditional & Conditional & Deal rates \\
\hline & (1) & (2) & (3) \\
\hline Intake & $\begin{array}{c}0.595 \\
(0.467)\end{array}$ & $\begin{array}{c}0.590 \\
(0.600)\end{array}$ & $\begin{array}{c}0.0374 \\
(0.0420)\end{array}$ \\
\hline Pie size & $\begin{array}{l}0.610^{* * *} \\
(0.0292)\end{array}$ & $\begin{array}{l}0.684^{* * *} \\
(0.0258)\end{array}$ & $\begin{array}{l}0.0146^{* * *} \\
(0.00358)\end{array}$ \\
\hline Bargaining round & $\begin{array}{c}0.172^{*} \\
(0.0979)\end{array}$ & $\begin{array}{c}0.124^{* *} \\
(0.0517)\end{array}$ & $\begin{array}{c}0.0110 \\
(0.0109)\end{array}$ \\
\hline Initial Position Partner & $\begin{array}{c}-0.290^{* * *} \\
(0.0686)\end{array}$ & $\begin{array}{c}-0.190^{* * *} \\
(0.0638)\end{array}$ & $\begin{array}{l}-0.0183^{* *} \\
(0.00721)\end{array}$ \\
\hline Risk Aversion & $\begin{array}{l}-0.120 \\
(0.112)\end{array}$ & $\begin{array}{c}-0.203 \\
(0.119)\end{array}$ & $\begin{array}{c}0.0121 \\
(0.0115)\end{array}$ \\
\hline Social Preferences & $\begin{array}{l}0.268^{*} \\
(0.134)\end{array}$ & $\begin{array}{c}0.288 \\
(0.224)\end{array}$ & $\begin{array}{l}0.00431 \\
(0.0286)\end{array}$ \\
\hline Constant & $\begin{array}{c}-5.961^{* * *} \\
(2.106)\end{array}$ & $\begin{array}{l}-6.266^{*} \\
(3.512)\end{array}$ & $\begin{array}{c}0.458 \\
(0.452)\end{array}$ \\
\hline Observations & 310 & 226 & 310 \\
\hline R-squared & 0.577 & 0.832 & 0.0866 \\
\hline
\end{tabular}

Note: $* p<0.10, * * p<0.05, * * * p<0.01$. Standard errors are clustered at the matching group level (in parentheses). Post-estimation Bonferroni-corrected Wald tests for ovulation versus the menstrual and postmenstrual phases with the effect size and the corresponding p-values [in parentheses]. The dependent variable is unconditional payoffs in model 1, payoffs conditional on a deal in model 2, and deal rates in model 3. Controls for demographics (age, nationality, and educational background) are included in all three models. 
Table 4.23: Uninformed players' un/conditional payoffs and deal rates

\begin{tabular}{|c|c|c|c|}
\hline & $\frac{\text { Unconditional }}{(1)}$ & $\frac{\text { Conditional }}{(2)}$ & $\frac{\text { Deal rates }}{(3)}$ \\
\hline Intake & $\begin{array}{l}0.595^{* *} \\
(0.252)\end{array}$ & $\begin{array}{c}0.349 \\
(0.215)\end{array}$ & $\begin{array}{c}0.0563 \\
(0.0429)\end{array}$ \\
\hline Pie size & $\begin{array}{l}0.179^{* * *} \\
(0.0314)\end{array}$ & $\begin{array}{l}0.120^{* * *} \\
(0.0221)\end{array}$ & $\begin{array}{l}0.0190^{* * *} \\
(0.00447)\end{array}$ \\
\hline Bargaining round & $\begin{array}{l}0.160^{* * *} \\
(0.0534)\end{array}$ & $\begin{array}{l}0.0656^{* *} \\
(0.0271)\end{array}$ & $\begin{array}{c}0.00921 \\
(0.00893)\end{array}$ \\
\hline Initial Position Partner & $\begin{array}{l}0.552^{* * *} \\
(0.0728)\end{array}$ & $\begin{array}{l}0.706^{* * *} \\
(0.0385)\end{array}$ & $\begin{array}{l}-0.000152 \\
(0.00851)\end{array}$ \\
\hline Risk Aversion & $\begin{array}{c}0.0390 \\
(0.0580)\end{array}$ & $\begin{array}{l}-0.0354 \\
(0.0355)\end{array}$ & $\begin{array}{c}0.0115 \\
(0.00977)\end{array}$ \\
\hline Social Preferences & $\begin{array}{c}0.134^{* *} \\
(0.0594)\end{array}$ & $\begin{array}{l}-0.0185 \\
(0.0907)\end{array}$ & $\begin{array}{l}0.0278^{* *} \\
(0.0122)\end{array}$ \\
\hline Constant & $\begin{array}{l}-0.0785 \\
(1.701)\end{array}$ & $\begin{array}{c}-0.659 \\
(1.293)\end{array}$ & $\begin{array}{l}0.670^{*} \\
(0.327)\end{array}$ \\
\hline Observations & 379 & 270 & 379 \\
\hline R-squared & 0.458 & 0.766 & 0.110 \\
\hline
\end{tabular}

Note: $* p<0.10, * * p<0.05, * * * p<0.01$. Standard errors are clustered at the matching group level (in parentheses). Post-estimation Bonferroni-corrected Wald tests for ovulation versus the menstrual and postmenstrual phases with the effect size and the corresponding p-values [in parentheses]. The dependent variable is unconditional payoffs in model 1 , payoffs conditional on a deal in model 2 , and deal rates in model 3. Controls for demographics (age, nationality, and educational background) are included in all three models.

Table 4.24: Risk and Social Preferences

\begin{tabular}{|c|c|c|c|c|}
\hline & \multicolumn{2}{|c|}{ Informed player } & \multicolumn{2}{|c|}{ Uninformed player } \\
\hline & $\begin{array}{c}\text { (1) } \\
\text { Risk aversion }\end{array}$ & $\begin{array}{c}\text { (2) } \\
\text { Social Pref. }\end{array}$ & $\begin{array}{c}\text { (3) } \\
\text { Risk aversion }\end{array}$ & $\begin{array}{c}\text { (4) } \\
\text { Social Pref. }\end{array}$ \\
\hline Intake & $\begin{array}{c}0.990^{*} \\
(0.521)\end{array}$ & $\begin{array}{c}0.104 \\
(0.382)\end{array}$ & $\begin{array}{c}0.654^{*} \\
(0.366)\end{array}$ & $\begin{array}{l}0.787^{* *} \\
(0.379)\end{array}$ \\
\hline Constant & $\begin{array}{c}3.737 \\
(3.540)\end{array}$ & $\begin{array}{c}4.722 \\
(3.013)\end{array}$ & $\begin{array}{l}-0.865 \\
(2.310)\end{array}$ & $\begin{array}{c}7.234^{* * *} \\
(2.555)\end{array}$ \\
\hline Demographics & $\checkmark$ & $\checkmark$ & $\checkmark$ & $\checkmark$ \\
\hline $\begin{array}{l}\text { Observations } \\
\text { R-squared }\end{array}$ & $\begin{array}{c}310 \\
0.142\end{array}$ & $\begin{array}{c}310 \\
0.192\end{array}$ & $\begin{array}{c}379 \\
0.0995\end{array}$ & $\begin{array}{c}379 \\
0.108\end{array}$ \\
\hline
\end{tabular}

Note: $* p<0.10, * * p<0.05, * * * p<0.01$. Standard errors (in parentheses). The dependent variable for Models 1 and 3 is risk aversion and for Models 2 and 4 is the level of benevolence. The explanatory variables demographics are age, nationality, and educational background for both type of players. The explanatory dummy variable Intake which takes the value of one if the participant is in the intake phase and zero otherwise. Standard errors are clustered at matching group level. 


\section{A6. Instructions}

[Page 1]

\section{GENERAL INSTRUCTIONS}

Thank you for your participation in the experiment today. ${ }^{25}$ It is important for the research that you are not communicating with the other participants in any other way than detailed in the instructions. If you have a question, please raise your hand and one of the experimenters will come to your place to answer your question in private.

In the experiment you can earn money with the decisions you make. It is therefore in your interest that you carefully read the instructions. All your decisions are private and anonymous. That means that your decisions cannot be linked to your name and other participants will not be able to identify your decisions during or after the experiment.

Please keep your ID number until the end of the experiment. You will be asked to introduce your ID number on the screen at the end of the experiment.

The experiment today consists of three parts: PART A, PART B, and PART C. After the last part you will be asked to answer a short questionnaire. You will receive the instructions for each part at the beginning of the corresponding part. You can find the printed instructions for Part A on your desk. The instructions for Part B and Part $\mathrm{C}$ will appear on the screen once you reach the corresponding part.

You will receive $5.00 €$ for the participation in the experiment and additionally $€ 10.00$ for having provided the printed calendar. The additional earnings you can make will depend on your own decisions, decisions of other participants and random events. This will be different in the different parts and will be described in detail in the beginning of the corresponding part. In PART A your earnings can be negative depending on your and other participants' decisions. In case of negative earnings they will be subtracted from your earnings in the other parts of this experiment. Note that you can always avoid negative earnings with your own decisions.

In summary, your total earnings from this experiment will be equal to: $5.00 €$ show-up fee $+10.00 €$ bonus + earnings from PART A + earnings from PART B + earnings from PART C. Your earnings will be paid to you privately in cash at the end of the session.

[Page 2]

\section{PART A, Instructions}

PART A will consist of 10 rounds of bargaining with different other participants.

In each round, two participants bargain on how to split an amount of money, which we call the "pie-size." This pie-size can be different in different rounds.

In each round, one participant in a bargaining pair will be informed about

\footnotetext{
${ }^{25}$ The text font, the size and the appearance of images have been adapted from the original instruction version.
} 
the pie-size. This participant will be called the "informed participant". The other participant in a bargaining round will not be informed of the pie-size. This participant will be called the "uninformed participant".

Who will be an informed who will be an uninformed participant will be decided randomly by the computer at the beginning of Part A. An informed participant will keep this role throughout all 10 rounds. Equivalently, an uninformed participant will also keep this role throughout all 10 rounds.

At the beginning of each round an informed and an uninformed participant will be randomly matched to bargain over the pie.

Importantly, in each round, bargaining takes place over the uninformed participant's share of the pie. In case of an agreement the uninformed participant earns that share and the informed participant earns the pie-size minus the share of the uninformed participant.

In each round the size of the pie will be $4.00 €, 8.00 €, 12.00 €, 16.00 €$, $20.00 €$ or $24.00 €$. In each round each of these pie-sizes is equally likely to be chosen. The actual pie-size will appear on the top left corner of the informed participant's screen. The uninformed participant will not be informed of the pie-size.

Bargaining takes place via the computer screen. The two participants in a pair will negotiate by moving a cursor on a slider that represents values from $0.00 €$ to $24.00 €$ (in increments of $0.10 €$ ). Importantly, for both participants the amounts on the slider represent the uninformed participant's share. On the computer screen this will be indicated by "Your proposal for the other" in case you are in the role of the informed participant and "Your proposal for yourself" in case you are in the role of the uninformed participant.

In the beginning of each round, both participants in a pair will have to select their initial offers. Initial offers will not be seen by the other participant until both participants have confirmed their initial offers (see Figure 1). At the beginning of a round the cursor will appear on the slider only after you have clicked on the slider. Bargaining will start only after both participants have confirmed their initial offers. Once both participants in a pair have confirmed their initial offers, a simultaneous bargaining stage will start. In this stage both participants in a pair will see both sliders (see Figure 2).

As soon as both sliders appear on the screen there are 30 seconds left for bargaining and reaching an agreement. Bargaining takes place by using the mouse to select proposals for the uninformed participant. Clicking the mouse on different positions of the slider moves the cursor to these positions.

The remaining time for reaching and agreements is shown on the top right corner of the computer screen (see Figure 2).

An agreement is reached when both cursors are in the same position for 2 seconds or if both sliders are at the same position when the time is over. When both cursors are in the same position on the slider, a green line connecting the two sliders will appear on the screen (see Figure 3).

\section{Payment}

If an agreement is reached, the informed participant's payment is equal to the pie-size minus the agreed share for the uninformed participant. If the agreed 
A.

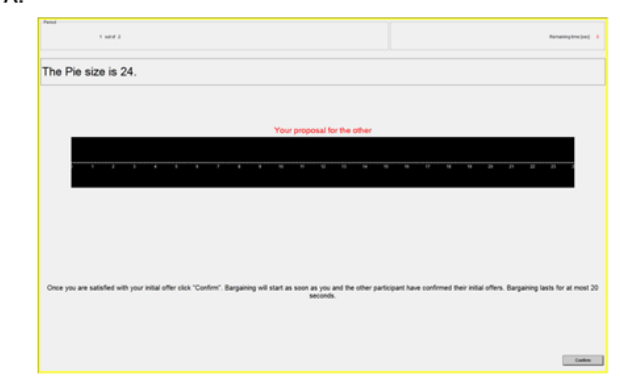

B.

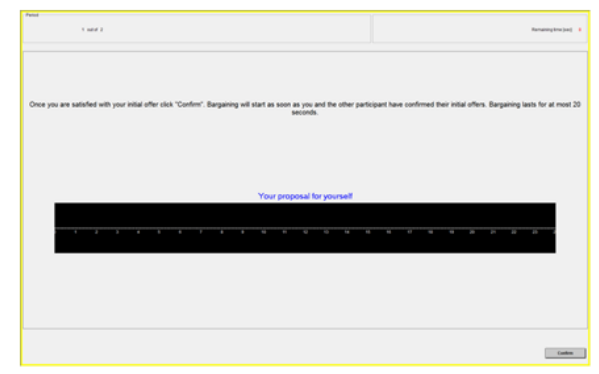

Figure 1: Screen for the initial offer/demand of the informed (A) and uninformed (B) player

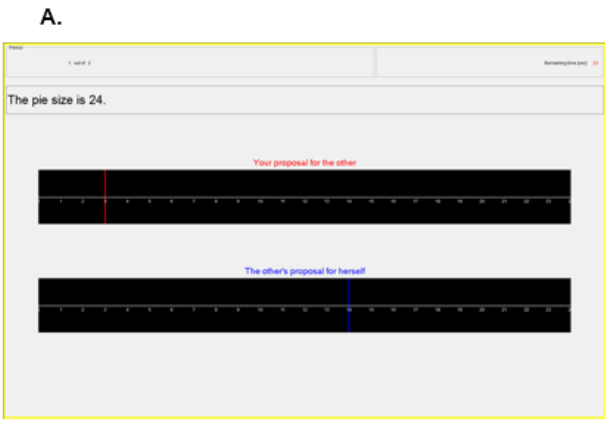

B.

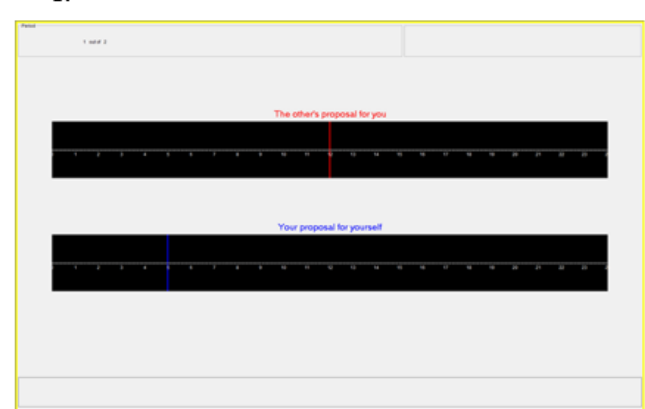

Figure 2: Simultaneous bargaining screen for the informed (A) and uninformed (B) player. This screen will show for 20 seconds.

share to the uninformed participant exceeds the pie-size, the payment of the informed participant will be negative. Negative payments will be subtracted from the other earnings in the experiment. 


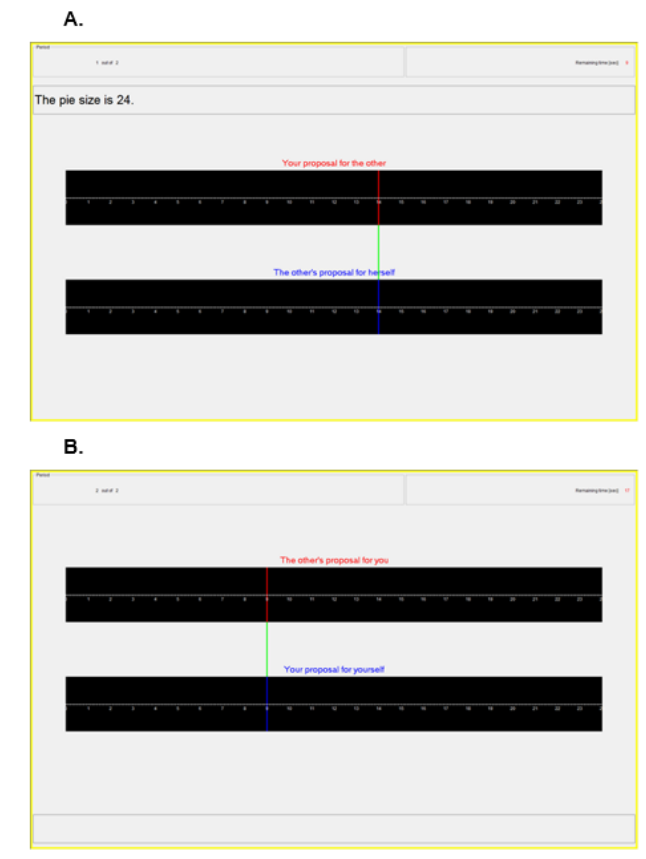

Figure 3: Agreement screen for the informed (A) and uninformed (B) player.

If no agreement is reached after 30 seconds of bargaining, both participants get $€ 0.00$.

After each round, both participants will be informed about the pie-size and their own payment (see Figure 4).

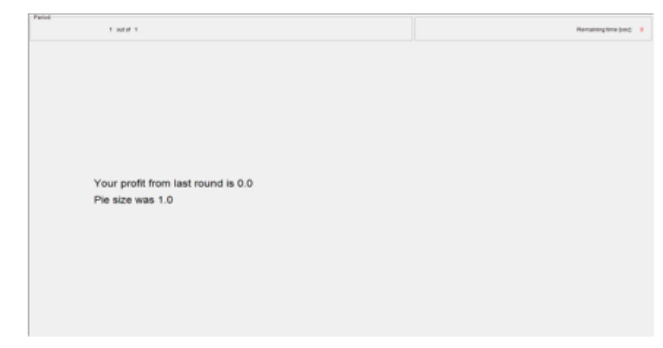

Figure 4: Payment screen for each participant.

PART A consists of 10 rounds of bargaining. Importantly, after each round participants will be rematched and it is unlikely that the same pair bargains in consecutive rounds.

\section{Earnings in PART A}

For your earnings in PART A, one of the 10 bargaining rounds will be randomly selected for being paid out. Each round is equally likely to be selected. Therefore you should view each round as the one that counts for your earnings in PART A. 


\section{PART B, Instructions}

\section{[Page 1]}

You are now going to make a series of decisions. These decisions will not influence your earnings from the first part of the experiment, nor will the decisions you made in the first part of the experiment influence the earnings from this part. Furthermore, the decisions you are going to make will only influence your own earnings.

You will be confronted with 10 decision situations. All these decision situations are completely independent of each other. A choice you made in one decision situation does not affect any of the other following decision situations.

Each decision situation is displayed on the screen. Each decision situation consists of a lottery (Option A), where you can earn a higher amount with some probability and a lower amount with some other probability and 10 sure amounts (Option B) represented in 10 rows. You have to decide for every row whether you prefer Option A (the lottery) or option B (the sure amount). Option A is the same for every row in a given decision situation, while option B takes 10 different values, one for each row. Note that within a decision situation you can only switch once: if you switch more than once a warning message will appear on the screen and you will be asked to change your decisions. By clicking on NEXT you will see an example screen of a decision situation.

\section{[Page 2]}

This is a screen shot of a typical decision situation that you are going to face. You are not asked to make choices now! Please have a careful look. Thereafter click on NEXT to proceed.

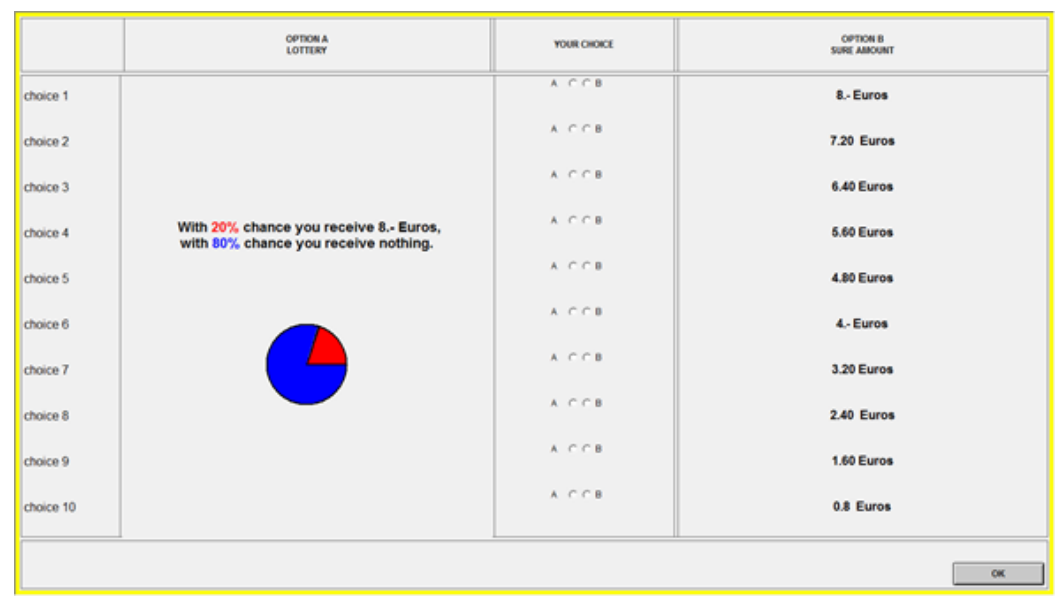

[Page 3] 


\section{Determination of earnings}

At the end of the experiment one of the 10 decision situations will be randomly selected with equal probability. Once the decision situation is selected, one of the 10 rows in this decision situation will be randomly selected with equal probability. The choice you will have made in this specific row will determine your earnings.

Consider, for instance, the screen shot that you just saw. Option A gives you a $20 \%$ chance to earn 8.- Euro and a $80 \%$ chance to earn nothing. Option B is always a sure amount that ranges from 8.- Euro in the first row, to 0.8 Euro in the 10th row. Suppose that the 8th row is randomly selected. If you would have selected option B, you would receive 2.40 Euro. If, instead, you would have selected option A, the outcome of the lottery determines your earnings. In that case the lottery would be played out by rolling a die. Depending on the outcome you would either earn 8,- Euro or 0,- Euro.

Please note that each decision situation has the same likelihood to be the one that is relevant for your earnings. Therefore, you should view each decision independently and consider all your choices carefully. At the end of the experiment, you will throw dice to determine which decision situation, which row in that decision situation, and possibly the lottery outcome will be paid out to you. You will throw dice under supervision of an experimenter.

If you like to, you can review the example screen once more by clicking on BACK. If you have any question please raise your hand. When you are ready, please press the BEGIN button below.

\section{PART C, Instructions}

\section{[ACTIVE ROLE]}

\section{[Page 1]}

This part of the experiment consists of another 10 decision situations. The decisions you make in this part will not influence your earnings from the first two parts of the experiment, nor will the decisions you made in the first two parts of the experiment influence the earnings from this part. The decisions you are going to make will influence your own earnings and the earnings of another person.

In each of the following 10 decision situations you are matched with another participant who remains anonymous to you and with whom you have not interacted before. We will refer to you as "active person" and to the other participant as "passive person." These use of terms will become clear below The passive person you are matched with is randomly determined by the computer.

In each decision situation you will have to choose between an "Alternative LEFT" and an "Alternative RIGHT". Each alternative has consequences for you and for the passive person you are matched with.

\section{Example of a decision situation}


In the example below you would have to choose between Alternative LEFT, in which you would get 2.00 Euro and the passive person would get 3.25 Euro, and Alternative RIGHT, in which you would get 2.50 Euro and the passive person would get 2.50 Euro. You make your decision by clicking on the LEFT or RIGHT button You are not asked to make choices now! Please have a careful look.

\begin{tabular}{|c|c|c|c|}
\hline Alternative LEFT & \multirow{2}{*}{\begin{tabular}{|c|} 
Your Choice \\
select here
\end{tabular}} & \multicolumn{2}{|c|}{ Alternative RIGHT } \\
\hline you get passive person gets & & you get & passive person gets \\
\hline 2.00 Euros 3.25 Euros & LEFT $\bigcirc \bigcirc$ RIGHT & 2.50 Euros & 2.50 Euros \\
\hline
\end{tabular}

\section{Determination of earnings}

Your earnings as active person in this part are determined as follows: At the end of the experiment, one of the 10 decision situations will be randomly chosen and the alternative chosen in this decision situation will be actually paid out. To determine the decision situation that is relevant for your earnings you will throw dice at the end of the experiment. If, for example, the randomly chosen decision task was the one shown above, and if in this task you had chosen Alternative RIGHT, then you as active person would receive 2.50 Euro, while the passive person you are matched with would receive 2.50 Euro. You will throw dice under supervision of an experimenter.

Recall that you have not interacted before with the passive person you are matched with in this part. Note also that each decision situation is equally likely to be relevant for your earnings and the earnings of the passive person. Therefore you should view each decision situation as the one that counts.

\section{[PASSIVE ROLE]}

\section{[Page 1]}

\section{PART C, Instructions (continued)}

You just made 10 choices as active person. The other participants also made choices in the same 10 decision situations. In addition to your earnings as active person, you will also earnings as passive person.

Your earnings as passive person in this part are determined as follows: In the exact same manner that the passive person you are matched with receives earnings from your decision, without having taken any action, you receive earnings from another participant without doing anything. That is you are the passive person of this other participant. This active person you are matched with as passive person is not the passive person you are matched with as 
active person. Moreover, you did not interact before with the active person you are matched with as passive person.

To determine the decision situation that counts for your earnings as passive person, the active person you are matched with will throw dice the same as you do as active person. In summary, your earnings from PART $C$ are determined by your earnings from the randomly selected decision task as active person plus your earnings from the randomly selected decision task as passive person. 


\section{Chapter 5}

\section{Conclusion}

This dissertation presents three experimental studies in competition and bargaining behavior. In particular, the first two studies focus on the discussion about whether preferences for competition exists, while the last study investigates the role of the menstrual cycle on women's bargaining behavior. Below I summarize the findings of each study.

Chapter 2, Is there a Preference for Competition? describes an experimental study where we build up on previous work to test whether a preference for competition exists by controlling for the role of risk preferences by design and measuring overconfidence carefully. We provide strong evidence for a preference for competition that it is highly consistent at the individual level and exists irrespective of risk attitudes. In addition, the results indicate that most of the participants have a defined preference for competition, as $45 \%$ are competition seeking and $30 \%$ are competition averse, and such preferences for competition have a small variation when confronted with different competition stakes. Our findings also reveal two more interesting patterns. First, people are more competition seeking in bigger groups as we observe that $51 \%$ of participants in a group of six people are competition seeking in contrast to $36 \%$ of participants that are competition seeking in a group of three people. Second, similar to Gillen et al. (2019) and van Veldhuizen (2017), we do not find gender differences in the willingness to compete. This last finding suggests that the common finding that men are more competitive seeking than women (Gneezy et al., 2003; Booth and Nolen, 2012; Dariel et al., 2017; Saccardo et al., 2018) vanishes when we control for risk preferences by design and confront participants with different competition stakes.

In Chapter 3, Estimating Preferences for Competition from Convex Budget Sets we conduct a laboratory experiment to test whether preferences for competition can be rationalized by a utility function and develop a framework for the joint treatment of preferences for competition and risk. This study provides the first evidence of consistency of individual choices with GARP in a competitive environment and provides structural individual-level estimates of preferences. Thanks to the features of our experimental design, we generate a 
rich data set that allows us to test for consistency of individual choices between a competitive and non-competitive environment, and to map such individual choices into a utility function that captures preferences for competition with two different interpretations. The first one assumes that preferences for competition affect directly payoffs in the utility function, and the second one allows preferences for competition to affect the utility function through risk preferences.

The estimates from our two interpretations suggest that participants are on average risk and competition averse. Interestingly, participants display less risk aversion behavior once we allow for an effect of preferences for competition on risk preferences. That is, when we account for the structural relation between risk and competitiveness simultaneously. This finding suggests that risk preferences differ between an environment with and without competition, and ignoring this relation might overestimate the level of risk aversion in the population. Lastly, in line with the literature in gender economics, we find that women are more risk and competition averse than men (Gneezy et al., 2003; Booth and Nolen, 2012; Dariel et al., 2017; Saccardo et al., 2018). Interestingly, the gender differences in risk aversion disappear once we allow for an effect of competition on risk preferences.

From the results discussed in Chapter 2 and Chapter 3, we conclude that the gender gap in competition and risk preferences is shaped by the relationship between these two traits. For instance, in Chapter 2 where we control for risk preferences by design, we do not observe gender differences in competition. In contrast, in Chapter 3 , we observe gender differences in preferences for competition when we account for the structural relationship between risk and competition. This suggests that when studying situations that involve competitive and risky behavior, one should carefully account for both preferences for competition and risk, and also for their interactions.

In Chapter 4, The Impact of the Menstrual Cycle on Bargaining Behavior I discuss a study where we experimentally examine the relationship between the menstrual cycle and bargaining behavior and outcomes. After a three months tracking period of the menstrual cycle, participants play in pairs a variation of the dynamic unstructured bilateral bargaining game with private information in Camerer et al. (2019). While the distribution of the pie size is common knowledge, only one informed partner knows the actual pie size. We find that the information setting plays an important role as only the bargaining behavior of informed women does vary across the menstrual cycle. Specifically, during the ovulation phase, informed women are less willing to compromise with the other bargaining partner compared to the other menstrual phases, and especially compared to the premenstrual phase. Furthermore, such a decrease in compromising behavior translates into significantly higher final payoffs during the ovulation phase. Lastly, although the bargaining behavior of uninformed women does not change over the menstrual cycle, their bargaining consequences captured by final payoffs and deal rates improve during ovulation. 


\section{Chapter 6}

\section{Impact Chapter}

In this dissertation, I use behavioral economics and rigorous experimental methods to study competitive and bargaining behavior. I also integrate insights from other disciplines such as psychology and biology. In addition to contributing to the academic discussion, my work also addresses important societal issues, especially in the context of labor markets and public policy. The research and findings presented in this dissertation can inform scientists and policymakers interested in (1) understanding competitive behavior, (2) making use of the portable tools to measure competitiveness (Chapters 2 and 3), (3) analyzing women's bargaining behavior and their biological foundations (Chapter 4), (4) as well as increasing the participation of women in top-level positions (Chapters 2, 3 and 4).

The studies "Is there a Preference for Competition?" covered in Chapter 2 and "Estimating Preferences for Competition from Convex Budget Sets" in Chapter 3, attempt to understand whether it is possible to conceptualize an individual preference for competition that is irrespective of risk attitudes. The findings from both studies suggest that individuals derive a direct taste from being in a competitive environment and that for a sizeable part of them this taste is negative (i.e., most individuals dislike taking part in a competitive environment compared to a non-competitive environment). There are two aspects that can be derived from this finding. First, there are agents who are forced to perform in competitive environments despite the discomfort they obtain from it, which can impair their accomplishments under this environment. Second, competitive environments have a selection of agents that like competition but not necessarily are the most qualified ones for it. In the labor market situation, both scenarios create inefficiencies, suggesting that policies that tackle competitive environments need to acknowledge and act upon them.

The findings from "Estimating Preferences for Competition from Convex Budget Sets" in Chapter 3 suggest that individuals experience different levels of risk aversion in a competitive and in a non-competitive environment. This variation across competitive environments can inform research on risk preferences, and 
more specifically, on the potential sources of variation of risk attitudes within individuals. Understanding the source of systematic changes in risk preferences is crucial, as such variations in risk behavior can have consequences in reallife situations. For instance, in labor market and health outcomes, migration decisions, addictive behavior, and investment (for review, see, e.g., SchildbergHörisch, 2018).

The methodologies used in Chapter 2 and in Chapter 3 come from the literature in experimental economics around gender differences in competitiveness. The findings from this literature propose that women are less willing to select into a competitive environment than men because they dislike competition more than men (for reviews, see, e.g., Niederle, 2014; Dariel et al., 2017). Although in both studies we observe that women on average dislike competition, it is not entirely clear whether the gender gap in competition is due to this distaste. Our results suggest that when risk is present in both a competitive and a non-competitive environment, men and women do not significantly differ in their aversion to competition. I consider that this finding can help to inform policies oriented to increase the representation of women in competitive environments and top-level positions. The fact that men and women seem to have similar preferences for competition, in general, suggests that other factors could play a more important role in gender differences in competitiveness. This could also explain why policy interventions such as information provision are so successful to reduce the gender gap in competition (Wozniak et al., 2014; Brandts et al., 2015; Balafoutas and Sutter, 2019). In addition to this type of policy intervention, targeting other traits such as risk attitudes, overconfidence, and other factors not present in my research (e.g., reputation and feedback aversion), could be more effective than targeting the trait of competition itself to increase women's participation.

Chapter 4, "The Impact of the Menstrual Cycle on Bargaining Behavior" proposes that the variation of bargaining behavior is conditional on the informational setting present in the negotiation process. Given the persisting gender gap both in terms of selection into negotiation and outcomes obtained from negotiations, I consider my findings can be informative to address this problem. In particular, the results in this chapter suggest that there are two forces influencing women's bargaining behavior: an environmental one and a biological one. The first force suggests the need for institutional changes that create better environmental conditions for women to negotiate. This is similar in the spirit to many other gender policies aiming to increase participation of women in the labor force such as quotas, prizes benefiting one's offspring, preferential treatments, and incentives based on cooperation. The second force highlights the need of creating awareness of the role that female sex hormones have in economic behavior. Scientific research analyzing the impact of the menstrual cycle on behavior is scarce not only in medical literature but also in behavioral studies. As a female young researcher it is shocking to see 
how little is known about internal processes that constantly shape women's bodies, and behavior in different social and economic domains. I think this study is not only informative for women to understand better how hormones can influence their negotiation decisions but also highlights the need for more research on this topic.

\section{Public debate and promotion of Maastricht University}

The research covered in this dissertation has been presented and discussed in several places such as Pittsburgh University (2020), New York University Abu Dhabi (2017-2019), New York University Shanghai (2019), and Universidad del Rosario (2017). In addition, my work has been presented in many prestigious behavioral and experimental conferences such as the ones hosted by the Economic Science Association (ESA) in Europe, America, and Asia, and other well-known conferences such as the International Meeting on Experimental and Behavioral Social Sciences (IMEBESS 2019), Gender Economics and The Workplace (IAB and FAU Erlangen-Nurenberg, Germany 2018), Maastricht Behavioral and Experimental Economics Symposium (M-BEES 2017) and Behavioral Economic Policy Symposium (M-BEPS 2017) and the Conference of Experimental Economics (BEEC, Bogota 2017).

\section{Software and Data analyses}

I used the software tool z-Tree to program all the experiments presented in this dissertation and the software STATA to perform the data analyses. The codes are available upon request to the author. 


\section{Curriculum Vitae}

Lina María Lozano Montaña was born on 24 of November, 1991 in Bogotá, Colombia. After finishing her high school at Colegio Refous in 2008, Lina wanted to combine the mathematical skills acquired during her early education with a social science formation. She decided to enroll in the program of Economics at Universidad del Rosario located in her hometown Bogotá. During her bachelor studies, Lina combined her studies with teaching activities to younger students in Economics and volunteering work with children from difficult households in Bogotá. She also obtained a scholarship to help financing her bachelor's studies thanks to her academic performance. With the help of the excellent professors at Universidad del Rosario, Lina discovered her passion for Economics and her wish to continue with a research path in this field. In 2014, Lina graduated with a Bachelor of Science in Economics, and in 2017 with a Research Master's Degree in Economics from Universidad del Rosario. During her Research Master's program, she worked as a research assistant at the Department of Economics at Universidad del Rosario focusing on education and cognitive development. In 2015, Lina received a scholarship from the government of Colombia to continue her education abroad, and decided to go for a more interdisciplinary road with a Research Master in Neuroeconomics at Maastricht University. In 2017, she became a $\mathrm{PhD}$ candidate at Maastricht University School of Business and Economics, under the supervision of Prof. Dr. Arno Riedl, Dr. Christina Rott (Vrije University) and Dr. Ernesto Reuben (NYU Abu Dhabi). During her PhD, Lina was a frequent visitor at the social science division at NYU Abu Dhabi hosted by Ernesto Reuben. She also has presented her work at various international conferences and seminars and participated in summer schools in Europe and Asia. In early 2020, she visited the Department of Economics at Pittsburgh University hosted by Lise Vesterlund. Starting in Fall 2021, Lina will be a postdoctoral researcher at New York University Abu Dhabi and also affiliated to the Center for Behavioral Institutional Design in the same institution. The results of her research are presented in this thesis. 


\section{Bibliography}

Abdellaoui, M., Bleichrodt, H., and Paraschiv, C. (2007). Loss aversion under prospect theory: A parameter-free measurement. Management Science, 53(10):1659-1674.

Afriat, S. N. (1972). Efficiency estimation of production functions. International Economic Review, pages 568-598.

Agranov, M. and Ortoleva, P. (2017). Stochastic choice and preferences for randomization. Journal of Political Economy, 125(1):40-68.

Almås, I., Cappelen, A. W., Salvanes, K. G., Sørensen, E. Ø., and Tungodden, B. (2016). What explains the gender gap in college track dropout? experimental and administrative evidence. American Economic Review, 106(5):296-302.

Andersen, S., Ertac, S., Gneezy, U., List, J. A., and Maximiano, S. (2013). Gender, competitiveness, and socialization at a young age: Evidence from a matrilineal and a patriarchal society. Review of Economics and Statistics, 95(4):1438-1443.

Andersen, S., Ertac, S., Gneezy, U., List, J. A., and Maximiano, S. (2018). On the cultural basis of gender differences in negotiation. Experimental Economics, 21(4):757-778.

Andersen, S., Harrison, G. W., Lau, M. I., and Rutström, E. E. (2008). Eliciting risk and time preferences. Econometrica, 76(3):583-618.

Andersen, S., Harrison, G. W., Lau, M. I., and Rutström, E. E. (2010). Preference heterogeneity in experiments: Comparing the field and laboratory. Journal of Economic Behavior \& Organization, 73(2):209-224.

Andersson, O., Holm, H. J., Tyran, J.-R., and Wengström, E. (2016). Risk aversion relates to cognitive ability: Preferences or noise? Journal of the European Economic Association, 14(5):1129-1154.

Andreoni, J. and Miller, J. (2002). Giving according to garp: An experimental test of the consistency of preferences for altruism. Econometrica, 70(2):737753.

Andreoni, J. and Sprenger, C. (2012). Estimating time preferences from convex budgets. American Economic Review, 102(7):3333-56. 
Apesteguia, J., Ballester, M. A., and Gutierrez, A. (2019). Random models for the joint treatment of risk and time preferences.

Apicella, C. L., Dreber, A., Campbell, B., Gray, P. B., Hoffman, M., and Little, A. C. (2008). Testosterone and financial risk preferences. Evolution and human behavior, 29(6):384-390.

Babcock, L. and Laschever, S. (2009). Women don't ask: Negotiation and the gender divide. Princeton University Press.

Balafoutas, L. and Sutter, M. (2019). How uncertainty and ambiguity in tournaments affect gender differences in competitive behavior. European Economic Review, 118:1-13.

Baran, N. M., Sapienza, P., and Zingales, L. (2010). Can we infer social preferences from the lab? evidence from the trust game. Technical report, National Bureau of Economic Research.

Benz, M. and Meier, S. (2008). Do people behave in experiments as in the field?-evidence from donations. Experimental economics, 11(3):268-281.

Berge, L. I. O., Bjorvatn, K., Pires, A. J. G., and Tungodden, B. (2015). Competitive in the lab, successful in the field? Journal of Economic Behavior \& Organization, 118:303-317.

Blanco, M., Engelmann, D., Koch, A. K., and Normann, H.-T. (2010). Belief elicitation in experiments: is there a hedging problem? Experimental Economics, 13(4):412-438.

Blau, F. D. and Kahn, L. M. (2000). Gender differences in pay. Journal of Economic perspectives, 14(4):75-99.

Bolton, G. E. and Karagözoğlu, E. (2016). On the influence of hard leverage in a soft leverage bargaining game: The importance of credible claims. Games and Economic Behavior, 99:164-179.

Booth, A. and Nolen, P. (2009). Choosing to compete: How different are girls and boys? iza discussion papers 4027. Institute for the Study of Labor (IZA).

Booth, A. and Nolen, P. (2012). Choosing to compete: How different are girls and boys? Journal of Economic Behavior \& Organization, 81(2):542-555.

Boudreau, K. J., Lacetera, N., and Lakhani, K. R. (2011). Incentives and problem uncertainty in innovation contests: An empirical analysis. Management Science, 57(5):843-863.

Brandts, J., Groenert, V., and Rott, C. (2015). The impact of advice on women's and men's selection into competition. Management Science, 61(5):10181035.

Bröder, A. and Hohmann, N. (2003). Variations in risk taking behavior over the menstrual cycle: An improved replication. Evolution and Human Behavior, 24(6):391-398. 
Bronars, S. G. (1987). The power of nonparametric tests of preference maximization. Econometrica: Journal of the Econometric Society, pages 693698.

Burnham, T. C. (2007). High-testosterone men reject low ultimatum game offers. Proceedings of the Royal Society B: Biological Sciences, 274(1623):2327-2330.

Buser, T. (2011). Hormones and social preferences. Technical report, Tinbergen Institute Discussion Paper.

Buser, T. (2012). The impact of the menstrual cycle and hormonal contraceptives on competitiveness. Journal of Economic Behavior \& Organization, 83(1):1-10.

Buser, T., Niederle, M., and Oosterbeek, H. (2014). Gender, competitiveness, and career choices. The Quarterly Journal of Economics, 129(3):1409-1447.

Buser, T., Niederle, M., and Oosterbeek, H. (2020). Can competitiveness predict education and labor market outcomes? evidence from incentivized choice and survey measures. Tinbergen Institute Discussion Paper 2020-048/I, Available at SSRN: https://ssrn.com/abstract $=3671348$.

Buser, T., Peter, N., and Wolter, S. C. (2017a). Gender, competitiveness, and study choices in high school: Evidence from switzerland. American Economic Review, 107(5):125-30.

Buser, T., Peter, N., and Wolter, S. C. (2017b). Gender, willingness to compete and career choices along the whole ability distribution.

Camerer, C. F. (1989). Does the basketball market believe in the 'Hot Hand,'? The American Economic Review, 79(5):1257-1261.

Camerer, C. F., Nave, G., and Smith, A. (2019). Dynamic unstructured bargaining with private information: theory, experiment, and outcome prediction via machine learning. Management Science, 65(4):1867-1890.

Cettolin, E., Riedl, A., and Tran, G. (2017). Giving in the face of risk. Journal of risk and uncertainty, 55(2-3):95-118.

Chavanne, T. J. and Gallup Jr, G. G. (1998). Variation in risk taking behavior among female college students as a function of the menstrual cycle. Evolution and Human Behavior, 19(1):27-32.

Che, Y.-K. and Gale, I. (2003). Optimal design of research contests. American Economic Review, 93(3):646-671.

Chen, Y., Katušcák, P., and Ozdenoren, E. (2013). Why can [U+02BC] t a woman bid more like a man? Games and Economic Behavior, 77(1):181-213.

Choi, S., Fisman, R., Gale, D., and Kariv, S. (2007). Consistency and heterogeneity of individual behavior under uncertainty. American Economic Review, 97(5):1921-1938. 
Christin-Maitre, S. (2013). History of oral contraceptive drugs and their use worldwide. Best practice \& research Clinical endocrinology \& metabolism, 27(1):3-12.

Chuang, Y. and Schechter, L. (2015). Stability of experimental and survey measures of risk, time, and social preferences: A review and some new results. Journal of development economics, 117:151-170.

Cohn, A., Fehr, E., and Goette, L. (2015). Fair wages and effort provision: Combining evidence from a choice experiment and a field experiment. Management Science, 61(8):1777-1794.

Creinin, M. D., Keverline, S., and Meyn, L. A. (2004). How regular is regular? an analysis of menstrual cycle regularity. Contraception, 70(4):289-292.

Croson, R. and Gneezy, U. (2009). Gender differences in preferences. Journal of Economic Literature, 47(2):448-74.

Danz, D., Vesterlund, L., and Wilson, A. J. (2020). Belief elicitation: Limiting truth telling with information on incentives. Technical report, National Bureau of Economic Research.

Dariel, A., Kephart, C., Nikiforakis, N., and Zenker, C. (2017). Emirati women do not shy away from competition: Evidence from a patriarchal society in transition. Journal of the Economic Science Association, 3(2):121-136.

Darwin's, C. (1859). On the origin of species. published on, 24.

Dittrich, M., Knabe, A., and Leipold, K. (2014). Gender differences in experimental wage negotiations. Economic Inquiry, 52(2):862-873.

Dohmen, T., Falk, A., Huffman, D., and Sunde, U. (2010). Are risk aversion and impatience related to cognitive ability? American Economic Review, 100(3):1238-60.

Dohmen, T., Falk, A., Huffman, D., Sunde, U., Schupp, J., and Wagner, G. G. (2011). Individual risk attitudes: Measurement, determinants, and behavioral consequences. Journal of the European Economic Association, 9(3):522-550.

Dreber, A., von Essen, E., and Ranehill, E. (2014). Gender and competition in adolescence: task matters. Experimental Economics, 17(1):154-172.

Eckel, C., De Oliveira, A. C., and Grossman, P. J. (2008). Gender and negotiation in the small: are women (perceived to be) more cooperative than men? Negotiation Journal, 24(4):429-445.

Embrey, M., Hyndman, K., and Riedl, A. (2021). Bargaining with a residual claimant: An experimental study. Games and Economic Behavior, 126:335354. 
Ertac, S. and Szentes, B. (2011). The effect of information on gender differences in competitiveness: Experimental evidence. Technical report, Working paper.

Fallucchi, F., Nosenzo, D., and Reuben, E. (2020). Measuring preferences for competition with experimentally-validated survey questions. Journal of Economic Behavior \& Organization, 178:402-423.

Fischbacher, U. (2007). z-Tree: Zurich toolbox for ready-made economic experiments. Experimental Economics, 10(2):171-178.

Fisman, R., Jakiela, P., and Kariv, S. (2017). Distributional preferences and political behavior. Journal of Public Economics, 155:1-10.

Fisman, R., Kariv, S., and Markovits, D. (2007). Individual preferences for giving. American Economic Review, 97(5):1858-1876.

Flory, J. A., Gneezy, U., Leonard, K. L., and List, J. A. (2018). Gender, age, and competition: A disappearing gap? Journal of Economic Behavior \& Organization, 150:256-276.

Flory, J. A., Leibbrandt, A., and List, J. A. (2015). Do competitive workplaces deter female workers? a large-scale natural field experiment on job entry decisions. The Review of Economic Studies, 82(1):122-155.

Franzen, A. and Pointner, S. (2013). The external validity of giving in the dictator game. Experimental Economics, 16(2):155-169.

Freeman, R. B. and Gelber, A. M. (2010). Prize structure and information in tournaments: Experimental evidence. American Economic Journal: Applied Economics, 2(1):149-64.

Friedl, A., Ring, P., and Schmidt, U. (2017). Gender differences in ambiguity aversion under different outcome correlation structures. Theory and Decision, 82(2):211-219.

Gächter, S. and Renner, E. (2010). The effects of (incentivized) belief elicitation in public goods experiments. Experimental Economics, 13(3):364-377.

Gächter, S. and Riedl, A. (2005). Moral property rights in bargaining with infeasible claims. Management Science, 51(2):249-263.

Galinsky, A. D. and Mussweiler, T. (2001). First offers as anchors: the role of perspective-taking and negotiator focus. Journal of personality and social psychology, 81(4):657.

Garcia, S. M. and Tor, A. (2009). The n-effect: More competitors, less competition. Psychological Science, 20(7):871-877.

Gee, L. K. (2019). The more you know: information effects on job application rates in a large field experiment. Management Science, 65(5):2077-2094. 
Geraldes, D. (2020). Women dislike competing against men. Available at SSRN.

Geraldes, D., Riedl, A., and Strobel, M. (2011). Sex and performance under competition: Is there a stereotype threat shadow. Presentation to the European Economic Association \& Econometric Society, Oslo, pages 25-29.

Gillen, B., Snowberg, E., and Yariv, L. (2019). Experimenting with measurement error: Techniques with applications to the caltech cohort study. Journal of Political Economy, 127(4):1826-1863.

Gneezy, U., Leonard, K. L., and List, J. A. (2009). Gender differences in competition: Evidence from a matrilineal and a patriarchal society. Econometrica, 77(5):1637-1664.

Gneezy, U., Niederle, M., and Rustichini, A. (2003). Performance in competitive environments: Gender differences. The Quarterly Journal of Economics, 118(3):1049-1074.

Gneezy, U. and Rustichini, A. (2004). Gender and competition at a young age. American Economic Review, 94(2):377-381.

Golobof, A. and Kiley, J. (2016). The current status of oral contraceptives: progress and recent innovations. In Seminars in reproductive medicine, volume 34, pages 145-151. Thieme Medical Publishers.

Green, D. P., Ha, S. E., and Bullock, J. G. (2010). Enough already about "black box" experiments: Studying mediation is more difficult than most scholars suppose. The Annals of the American Academy of Political and Social Science, 628(1):200-208.

Greiner, B. (2015). Subject pool recruitment procedures: organizing experiments with orsee. Journal of the Economic Science Association, 1(1):114-125.

Haag, P., Hanhart, N., and M "u ller, M. (2016). Gyn "a ecology and urology for study and practice: incl. obstetrics, reproductive medicine, sexual medicine, andrology, etc. Venereology: taking into account the subject catalog and the oral exams in the "A medical examinations. Medical publishing and information services.

Hampson, E. and Young, E. A. (2008). Methodological issues in the study of hormone-behavior relations in humans: Understanding and monitoring the menstrual cycle. Sex differences in the brain: From genes to behavior, pages 63-78.

Hanek, K. J., Garcia, S. M., and Tor, A. (2016). Gender and competitive preferences: The role of competition size. Journal of Applied Psychology, 101(8):1122.

Harrison, G. W., Lau, M. I., and Rutström, E. E. (2007). Estimating risk attitudes in denmark: A field experiment. Scandinavian Journal of Economics, 109(2):341-368. 
Harrison, G. W. and Phillips, R. D. (2014). Subjective beliefs and statistical forecasts of financial risks: The chief risk officer project. In Contemporary Challenges in Risk Management, pages 163-202. Springer.

Harrison, G. W. and Rutström, E. E. (2008). Risk aversion in the laboratory. In Risk Aversion in Experiments. Emerald Group Publishing Limited.

Hausman, J. (2001). Mismeasured variables in econometric analysis: problems from the right and problems from the left. Journal of Economic Perspectives, 15(4):57-67.

Heckman, J. J., Jagelka, T., and Kautz, T. D. (2019). Some contributions of economics to the study of personality. Technical report, National Bureau of Economic Research.

Herbst, D. and Mas, A. (2015). Peer effects on worker output in the laboratory generalize to the field. Science, 350(6260):545-549.

Hey, J. D. and Orme, C. (1994). Investigating generalizations of expected utility theory using experimental data. Econometrica: Journal of the Econometric Society, pages 1291-1326.

Holt, C. A. and Laury, S. K. (2002). Risk aversion and incentive effects. American Economic Review, 92(5):1644-1655.

Hossain, T. and Okui, R. (2013). The binarized scoring rule. Review of Economic Studies, 80(3):984-1001.

Ifcher, J. and Zarghamee, H. (2016). Pricing competition: a new laboratory measure of gender differences in the willingness to compete. Experimental Economics, 19(3):642-662.

Iriberri, N. and Rey-Biel, P. (2012). Let's (not) talk about sex: Gender awareness and stereotype-threat on performance under competition. Citeseer.

Jones, B. C., Little, A. C., Boothroyd, L., DeBruine, L. M., Feinberg, D. R., Smith, M. L., Cornwell, R. E., Moore, F. R., and Perrett, D. I. (2005). Commitment to relationships and preferences for femininity and apparent health in faces are strongest on days of the menstrual cycle when progesterone level is high. Hormones and behavior, 48(3):283-290.

Jung, S. and Vranceanu, R. (2017). Experimental estimates of men's and women's willingness to compete: Does the gender of the partner matter?

Kamas, L. and Preston, A. (2018). Competing with confidence: The ticket to labor market success for college-educated women. Journal of Economic Behavior \& Organization, 155:231-252.

Karagözoğlu, E. and Riedl, A. (2015). Performance information, production uncertainty, and subjective entitlements in bargaining. Management Science, 61(11):2611-2626. 
Karlan, D. S. (2005). Using experimental economics to measure social capital and predict financial decisions. American Economic Review, 95(5):16881699.

Karni, E. (2009). A mechanism for eliciting probabilities. Econometrica, 77(2):603-606.

Kerschbamer, R. (2015). The geometry of distributional preferences and a nonparametric identification approach: The equality equivalence test. European economic review, 76:85-103.

Kettlewell, N. (2019). Risk preference dynamics around life events. Journal of Economic Behavior \& Organization, 162:66-84.

Kosfeld, M., Heinrichs, M., Zak, P. J., Fischbacher, U., and Fehr, E. (2005). Oxytocin increases trust in humans. Nature, 435(7042):673-676.

Kray, L. J., Thompson, L., and Galinsky, A. (2001). Battle of the sexes: gender stereotype confirmation and reactance in negotiations. Journal of personality and social psychology, 80(6):942.

Lazzaro, S. C., Rutledge, R. B., Burghart, D. R., and Glimcher, P. W. (2016). The impact of menstrual cycle phase on economic choice and rationality. PLoS One, 11(1): 0144080.

Leibbrandt, A., Gneezy, U., and List, J. A. (2013). Rise and fall of competitiveness in individualistic and collectivistic societies. Proceedings of the National Academy of Sciences, 110(23):9305-9308.

Leibbrandt, A. and List, J. A. (2015). Do women avoid salary negotiations? evidence from a large-scale natural field experiment. Management Science, 61(9):2016-2024.

Lovett, J. L., Chima, M. A., Wexler, J. K., Arslanian, K. J., Friedman, A. B., Yousif, C. B., and Strassmann, B. I. (2017). Oral contraceptives cause evolutionarily novel increases in hormone exposurea risk factor for breast cancer. Evolution, medicine, and public health, 2017(1):97-108.

Mata, R., Frey, R., Richter, D., Schupp, J., and Hertwig, R. (2018). Risk preference: A view from psychology. Journal of Economic Perspectives, 32(2):155-72.

Mayr, U., Wozniak, D., Davidson, C., Kuhns, D., and Harbaugh, W. T. (2012). Competitiveness across the life span: the feisty fifties. Psychology and Aging, 27(2):278.

Meissner, T., Gassmann, X., Faure, C., and Schleich, J. (2020). Individual characteristics associated with risk and time preferences-a multi-country representative survey. Working paper.

Niederle, M. (2014). Gender. NBER Working Paper Series. doi:10.3386/w20788. 
Niederle, M. (2015). Gender. handbook of experimental economics.

Niederle, M. and Vesterlund, L. (2007). Do women shy away from competition? do men compete too much? The quarterly journal of economics, 122(3):10671101.

Niederle, M. and Vesterlund, L. (2011). Gender and competition. Annu. Rev. Econ., 3(1):601-630.

Okudaira, H., Kinari, Y., Mizutani, N., Ohtake, F., and Kawaguchi, A. (2015). Older sisters and younger brothers: The impact of siblings on preference for competition. Personality and Individual Differences, 82:81-89.

Pearson, M. and Schipper, B. C. (2013). Menstrual cycle and competitive bidding. Games and Economic Behavior, 78:1-20.

Penton-Voak, I. S. and Perrett, D. I. (2000). Female preference for male faces changes cyclically: Further evidence. Evolution and Human Behavior, 21(1):39-48.

Petrie, R. and Segal, C. (2015). Gender differences in competitiveness: The role of prizes.

Potters, J. and Stoop, J. (2016). Do cheaters in the lab also cheat in the field? European Economic Review, 87:26-33.

Ranehill, E., Zethraeus, N., Blomberg, L., von Schoultz, B., Hirschberg, A. L., Johannesson, M., and Dreber, A. (2018). Hormonal contraceptives do not impact economic preferences: Evidence from a randomized trial. Management Science, 64(10):4515-4532.

Reuben, E., Sapienza, P., and Zingales, L. (2015). Taste for competition and the gender gap among young business professionals. NBER Working paper No. 21695 doi: 10.3386/w21695.

Reuben, E., Wiswall, M., and Zafar, B. (2017). Preferences and biases in educational choices and labour market expectations: Shrinking the black box of gender. The Economic Journal, 127(604):2153-2186.

Riedl, A. and Smeets, P. (2017). Why do investors hold socially responsible mutual funds? The Journal of Finance, 72(6):2505-2550.

Rutström, E. E. and Wilcox, N. T. (2009). Stated beliefs versus inferred beliefs: A methodological inquiry and experimental test. Games and Economic Behavior, 67(2):616-632.

Saccardo, S., Pietrasz, A., and Gneezy, U. (2018). On the size of the gender difference in competitiveness. Management Science, 64(4):1541-1554.

Salamanca, N. (2018). The dynamic properties of economic preferences.

Schildberg-Hörisch, H. (2018). Are risk preferences stable? Journal of Economic Perspectives, 32(2):135-54. 
Schipper, B. C. (2014). Sex hormones and choice under risk. Available at SSRN 2046324.

Small, D. A., Gelfand, M., Babcock, L., and Gettman, H. (2007). Who goes to the bargaining table? the influence of gender and framing on the initiation of negotiation. Journal of personality and social psychology, 93(4):600.

Snowberg, E. and Yariv, L. (2021). Testing the waters: Behavior across participant pools. American Economic Review, 111(2):687-719.

Stricker, R., Eberhart, R., Chevailler, M.-C., Quinn, F. A., Bischof, P., and Stricker, R. (2006). Establishment of detailed reference values for luteinizing hormone, follicle stimulating hormone, estradiol, and progesterone during different phases of the menstrual cycle on the abbott architect ${ }^{\circledR}$ analyzer. Clinical Chemistry and Laboratory Medicine (CCLM), 44(7):883-887.

Trautmann, S. T. and van de Kuilen, G. (2015). Belief elicitation: A horse race among truth serums. The Economic Journal, 125(589):2116-2135.

Tversky, A. (1969). Intransitivity of preferences. Psychological Review, 76(1):31.

Van Lange, P. A., Schippers, M., and Balliet, D. (2011). Who volunteers in psychology experiments? an empirical review of prosocial motivation in volunteering. Personality and Individual Differences, 51(3):279-284.

van Veldhuizen, R. (2017). Gender differences in tournament choices: Risk preferences, overconfidence or competitiveness? NBER Working paper No. 21695 doi: 10.3386/w21695.

Varian, H. R. (1982). The nonparametric approach to demand analysis. Econometrica: Journal of the Econometric Society, pages 945-973.

Varian, H. R. et al. (1991). Goodness-of-fit for revealed preference tests. Citeseer.

Wakker, P. P. (2008). Explaining the characteristics of the power (crra) utility family. Health economics, 17(12):1329-1344.

Wang, S. W. (2011). Incentive effects: The case of belief elicitation from individuals in groups. Economics Letters, 111(1):30-33.

Westfall, J. and Yarkoni, T. (2016). Statistically controlling for confounding constructs is harder than you think. PloS One, 11(3):e0152719.

Wozniak, D., Harbaugh, W. T., and Mayr, U. (2009). Choices about competition: Differences by gender and hormonal fluctuations, and the role of relative performance feedback. Available at SSRN 1564895.

Wozniak, D., Harbaugh, W. T., and Mayr, U. (2014). The menstrual cycle and performance feedback alter gender differences in competitive choices. Journal of Labor Economics, 32(1):161-198.

Wozniak, D., Harbaugh, W. T., and Mayr, U. (2016). The effect of feedback on gender differences in competitive choices. Available at SSRN 1976073. 
Zethraeus, N., Kocoska-Maras, L., Ellingsen, T., von Schoultz, B., Hirschberg, A. L., and Johannesson, M. (2009). A randomized trial of the effect of estrogen and testosterone on economic behavior. Proceedings of the National Academy of Sciences, 106(16):6535-6538.

Zhang, Y. J. (2019). Culture, institutions and the gender gap in competitive inclination: Evidence from the communist experiment in china. The Economic Journal, 129(617):509-552. 